\title{
Predicting adverse outcomes in older medical emergency department patients
}

Citation for published version (APA):

Zelis, N. (2020). Predicting adverse outcomes in older medical emergency department patients. [Doctoral Thesis, Maastricht University]. Gildeprint Drukkerijen. https://doi.org/10.26481/dis.20201216nz

Document status and date:

Published: 01/01/2020

DOI:

10.26481/dis.20201216nz

Document Version:

Publisher's PDF, also known as Version of record

\section{Please check the document version of this publication:}

- A submitted manuscript is the version of the article upon submission and before peer-review. There can be important differences between the submitted version and the official published version of record.

People interested in the research are advised to contact the author for the final version of the publication, or visit the DOI to the publisher's website.

- The final author version and the galley proof are versions of the publication after peer review.

- The final published version features the final layout of the paper including the volume, issue and page numbers.

Link to publication

\footnotetext{
General rights rights.

- You may freely distribute the URL identifying the publication in the public portal. please follow below link for the End User Agreement:

www.umlib.nl/taverne-license

Take down policy

If you believe that this document breaches copyright please contact us at:

repository@maastrichtuniversity.nl

providing details and we will investigate your claim.
}

Copyright and moral rights for the publications made accessible in the public portal are retained by the authors and/or other copyright owners and it is a condition of accessing publications that users recognise and abide by the legal requirements associated with these

- Users may download and print one copy of any publication from the public portal for the purpose of private study or research.

- You may not further distribute the material or use it for any profit-making activity or commercial gain

If the publication is distributed under the terms of Article $25 \mathrm{fa}$ of the Dutch Copyright Act, indicated by the "Taverne" license above, 
Predicting adverse outcomes in older medical emergency department patients

Noortje Zelis 


\section{Colofon}

Copyright (c) Noortje Zelis, 2020. All rights reserved.

Cover: Jean Scheijen | vierdrie.nl

Lay-out: Tiny Wouters-Lenssen

Production: Gildeprint B.V.

ISBN: 9789464190854

Financial support by Maastricht University. 


\title{
Predicting adverse outcomes in older medical emergency department patients
}

\author{
PROEFSCHRIFT \\ ter verkrijging van de graad van doctor aan de Universiteit Maastricht, \\ op gezag van de Rector Magnificus, Prof. Dr. Rianne M. Letschert, \\ volgens het besluit van het College van Decanen, \\ in het openbaar te verdedigen \\ woensdag 16 december 2020 om 12.00 uur
}

door

Noortje Zelis

Geboren op 12 december 1985 te Heerlen 


\section{Promotor}

Prof. dr. P.W. de Leeuw

\section{Copromotores}

Dr. P.M. Stassen

Dr. J. Buijs

\section{Beoordelingscommissie}

Prof. dr. J.M.G.A. Schols (voorzitter)

Prof. dr. O. Bekers

Prof. dr. F.H. Bosch, Radboud Universiteit, Nijmegen

Prof. dr. W.F. Buhre

Prof. dr. B.M. Buurman-van Es, Universiteit van Amsterdam 


\section{Table of contents}

$\begin{array}{lll}\text { Chapter } 1 & \text { General Introduction } & 7\end{array}$

Chapter 2 Study protocol for a multicentre prospective cohort study to 19 identify predictors of adverse outcome in older medical emergency department patients (the Risk Stratification in the Emergency Department in Acutely III Older Patients (RISE UP) study)

Chapter 3 Short-term mortality in older medical emergency patients can be predicted using clinical intuition: A prospective study

Chapter 4 Concerns of older patients and their caregivers in the emergency department

Chapter 5 A new simplified model for predicting 30-day mortality in older medical emergency department patients: The RISE UP score

Chapter 6 Biomarkers do not add to the RISE UP score in predicting mortality in older medical emergency department patients

Chapter 7 Major adverse cardiovascular events in older emergency department patients presenting with non-cardiac medical complaints

Chapter 8 The RISE UP score: predicting prognosis in patients with

COVID-19 in the emergency department

Chapter 9 Summary and general discussion

Samenvatting

Impact paragraph

Dankwoord

Curriculum Vitae

Scientific output 

Chapter 1

General introduction 



\section{Introduction}

With growing life expectancy and rapid ageing of the population, more and more older people contact medical health services worldwide. As a result, the number of emergency department (ED) visits by older patients is rising and is expected to increase even more over the following decades. In 2019, there were 703 million persons aged 65 years or more worldwide, and by 2050, this number is expected to double to more than 1.5 billion. $^{1}$ Besides this absolute increase, the proportion of older people is expected to increase globally from 6\% in 2019 to $16 \%$ in 2050 . In the Netherlands the number of older persons is likely to grow from 3.4 million in 2020 $( \pm 20 \%)$ to 4.9 million in $2050( \pm 25 \%)$, with older persons far outnumbering persons aged 20 years or younger. ${ }^{2}$

In 2017 patients aged 65 years or older were responsible for approximately one third of all ED visits in The Netherlands. ${ }^{3}$ Especially patients of 80 years or older visit EDs most frequently. ${ }^{4}$ EDs play a central role in the acute care of older patients and while EDs were traditionally designed to treat single acute medical conditions, with rising ED visits by older patients, ED care is becoming more and more complex. ${ }^{5}$

\section{Older emergency department patients, their experiences and risk of adverse outcomes}

During an ED visit, important decisions concerning diagnostic tests, treatment intensity and restrictions have to be made within a short time frame. Older patients frequently present to the ED with medical (i.e. non-surgical) problems and, compared to younger patients, they more often arrive by ambulance and with a higher level of urgency. ${ }^{6,7}$ Decision making during ED stay is challenged by ED crowding and multiple interruptions of the doctor during the clinical assessment of the (unfamiliar) patient. Particularly in older patients, decision making is difficult due to the interaction between the noisy, crowded ED environment and geriatric factors such as cognitive impairment, delirium and impaired mobility, vision and/or hearing. In addition, these older ED patients often present with complex medical problems and non-specific complaints, requiring a large number of diagnostic tests, procedures and need of personalised care. This results frequently in a prolonged ED stay. ${ }^{6,8,9}$

The result of the urgent and complex decision making concerning older ED patients, is that diagnoses are likely to be less accurate than in younger patients, despite of extensive ancillary investigations frequently performed. ${ }^{6,7}$ Atypical and complex 
clinical presentation, undertriage, comorbidity, altered mental state, communication problems and obscure medication use are all factors that contribute to the difficult assessment, decision making and to missed diagnoses in older ED patients and may lead to a higher risk of adverse outcomes.

For many older patients, an ED visit is an impressive event. Long waiting times, unfamiliarity with ED processes and the uncertainty about what might be the problem and what will happen may result in a lot of anxiety during ED stay. ${ }^{10-12}$ Only little is known about the concerns of older ED patients, while insight in their concerns may offer an opportunity to make care more person-centred.

Apart from the possible psychological impact of the ED visit, older patients visiting the ED are at high risk of adverse outcomes, including mortality. ${ }^{6,713}$ Older ED patients are more frequently hospitalised, admitted to an Intensive Care Unit (ICU) and readmitted to the hospital, compared to younger patients. Within 3 months, approximately $25 \%$ of older patients revisit the ED and approximately $10-45 \%$ experience functional decline. ${ }^{6.14}$ Most older patients experience new disabilities in activities of daily living (i.e. functional decline) in the two weeks before ED presentation, which further increases during hospitalisation. ${ }^{14}$ After hospital discharge, most older patients never return to their prior functional capacity and experience a permanent loss of function. Mortality is high in older ED patients and is approximately $10 \%$ within 3 months after the ED visit, and even $24 \%$ in patients presenting with medical problems. ${ }^{15}$ The major causes of death in older patients ( $\geq 65$ years) in the US and Europe are ischemic heart disease, respiratory diseases, cerebrovascular diseases, cancer and Alzheimer's disease. ${ }^{16,17}$ Despite of the fact that cardiovascular diseases still remain the world's leading cause of death in older patients, the risk of major adverse cardiovascular events (MACE) following an ED visit in older patients who present with non-cardiac problems is still unknown.

The increasing number of ED visits, the complex and time-consuming assessment and difficult decision making together with the high risk of adverse outcomes make it highly important to focus on risk factors for adverse outcome and on experiences of older ED patients during their ED visit in order to improve decision making, care and outcome. 


\section{Prediction of adverse outcomes in older emergency department patients}

In recent years, more attention is being paid to early identification of older ED patients at high risk of adverse outcomes ${ }^{18,19}$, in an attempt to improve clinical outcomes and well-being. Identification of high risk older patients is crucial, in order to personalise and improve medical care ${ }^{20}$ and to avoid unnecessary treatments. Especially for older patients, decisions on treatment are strongly influenced by their mortality risk ${ }^{21,22}$ and since prognosis plays such a central role in clinical decision making, prediction of adverse outcomes may lead to a more personalized decision making and improvement of care and outcomes in older ED patients.

Patients at high risk of adverse outcome may be identified in several stages during their ED visit (Figure 1.1). In the first stage of the ED visit, patients can be identified by the nurse during triage and by the clinical intuition of patients, caregivers, nurses and physicians. In the second stage of the ED visit, e.g. after the first assessment (history taking and physical examination) of the patient, routine laboratory tests, markers of specific diseases (i.e. biomarkers) and risk-stratification scores, including clinical prediction models, can be used. Nevertheless, only a limited number of these tools are validated in older patients and most have suboptimal discriminatory value, leading to inadequate recognition of high-risk patients. ${ }^{18,23,24}$ In addition, many of the riskstratification scores, designed for older patients, use information which is difficult to retrieve during the acute phase of an ED visit (e.g. cognitive impairment or functional status).

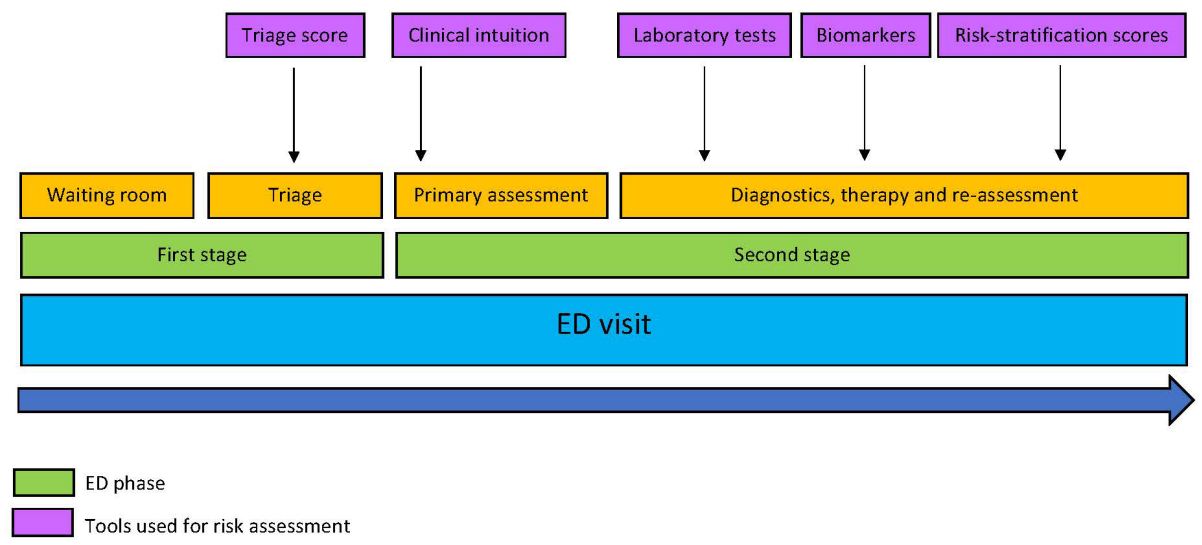

Figure 1.1 Overview of early risk-assessment tools during different phases of the ED visit. 
When a patient is found to be at high risk of adverse outcomes a comprehensive geriatric assessment (CGA), which assesses specific problems in multiple domains including cognitive, physical, somatic, psychological and social functioning, can be applied. This CGA is used to develop a coordinated and integrated plan for treatment and long term follow up and can improve outcome in older patients after an acute hospital admission. ${ }^{25}$ However, this CGA is time-consuming and can therefore not be used routinely in older ED patients.

\section{Triage scores}

Triage systems aim to identify patients at risk for deterioration and are applied to identify patients who should be assessed and treated immediately. Triage scores can also be used to predict clinical outcomes. Still, older patients are at risk of undertriage by these systems, probably because they have non-specific complaints and multiple diseases presenting in an atypical way. ${ }^{26}$ One of these triage systems is the Manchester Triage System (MTS) ${ }^{27}$, which also tends to undertriage older patients ${ }^{28}$ and has low discriminatory ability for predicting mortality in older ED patients. ${ }^{29}$

\section{Clinical intuition}

Another way to identify patients at risk of adverse outcome may be the use of clinical intuition, i.e. the clinical impression of nurses and physicians and disease perception or self-rated health of patients. ${ }^{15,30-36}$ The surprise question: "Would I be surprised if this patient died in the next 12 months?" can be used to predict mortality. ${ }^{37,38}$ Most of the studies investigating clinical intuition show moderate discriminatory values for adverse outcomes. However, most of these studies were performed in young or selected populations (e.g. patients with cancer, sepsis, end-stage renal disease or nonspecific complaints) and in other clinical settings (e.g. admission units or ICUs) and may therefore not be representative for the situation of older ED patients. ${ }^{15,31-34,37}$ The first clinical impression, i.e. the first impression before history taking and physical examination, was hardly ever tested, since in most of the studies professionals had access to the results of physical examination and diagnostics. Especially this first clinical impression may be very important, because this impression will guide the initial diagnostic and treatment approach in ED patients.

\section{Commonly used laboratory tests and biomarkers}

Laboratory tests are used more or less routinely in ED patients for diagnostic or prognostic reasons. Especially in older medical ED patients, a more extensive package 
of laboratory tests may be applied. Routine laboratory tests have been shown to predict mortality in ED and hospitalised patients. ${ }^{39-41}$ In addition, non-routine laboratory tests (i.e. biomarkers) are used frequently in ED patients to identify or quantify the severity of specific diseases. Lactate, high-sensitivity cardiac troponin T (hs-cTnT), N-terminal pro-B-type natriuretic peptide (NT-proBNP), d-dimer and procalcitonin (PCT) are such biomarkers, frequently ordered in medical ED patients. They are indicators of serious conditions and diseases, frequently present in older ED patients, including tissue hypoperfusion, myocardial injury, heart failure, thromboembolism and bacterial infection. Previous studies showed that these biomarkers are predictive for adverse outcomes. ${ }^{42-53}$ However, in these studies, biomarkers were only measured when deemed indicated by the ED physician, probably affecting their discriminatory value. ${ }^{42-45,47-49}$ In addition, most studies were performed in selected (e.g. patients with infection, sepsis, cardiac symptoms or nonspecific complaints) and in younger patients. ${ }^{42-45,47-49,51-53}$ Since laboratory tests and biomarkers are predictive of adverse outcomes in several settings, these tests may be used as tools to predict adverse outcome in older ED patients as well.

\section{Risk stratification scores}

Risk stratification scores are often used to assess severity of disease, adapt treatment options or predict outcomes in ED patients. Some of these scores are developed for the general hospital population and are not disease-specific, including the Acute Physiology and Chronic Health Evaluation II (APACHE II) score ${ }^{54}$ and the Modified Early Warning Score (MEWS). ${ }^{55}$ The APACHE II score assigns points to 12 physiological variables, age and the previous health status and is used in ICU patients to predict mortality. The APACHE II score is not always feasible, due to the incorporation of blood gas analysis. Furthermore, this score is not validated in older ED patients. The MEWS is used in the general ED and hospital population for prediction of clinical deterioration and mortality. Its discriminatory value for mortality in older ED patients has not been studied. Other risk stratification scores are specifically designed for the older ED population including the Identification of Seniors at Risk (ISAR) score ${ }^{56}$, the ISAR-Hospitalized Patients (ISAR-HP) score $^{57}$ and the acute presenting older patient (APOP) screener. ${ }^{19}$ These scores, however, have suboptimal discriminatory values

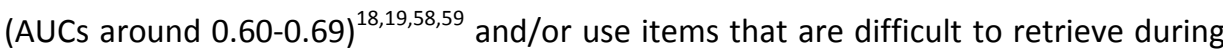
ED stay (e.g. premorbid cognitive or physical functioning) ${ }^{19,56,57}$, which makes them less applicable for ED use. In addition, the Clinical Frailty Scale (CFS), which was developed to identify frail older patients, can be used to predict short-term mortality with AUCs ranging from 0.72 to 0.81 in different studies. ${ }^{60-62}$ This score has recently 
been shown to predict short-term mortality in ED patients with corona virus disease 2019 (COVID-19) as well. ${ }^{63}$ Similar to the other geriatric scores, the CFS uses physical and cognitive functioning, which are often difficult to reliably retrieve during ED stay.

Simplicity and conciseness of a risk stratification score is found to be very important for successful implementation in daily practice. ${ }^{64}$ Most risk stratification scores and prediction models are used as a dichotomised test (i.e. either a high risk of adverse outcomes or not), while the risk of adverse outcomes is equivocal. ${ }^{65}$ Therefore, we state that it is very important to develop a reliable and easily applicable riskstratification score, which provides a percentage of risk, rather than a dichotomous output. This risk percentage can then be interpreted within the complex context of a patient's premorbid and morbid condition, in order to guide clinical decision making.

\section{Aims and outline of the present thesis}

To provide a better and more personalized care for older ED patients and in order to improve their clinical outcome and well-being, it is highly important that we characterize these older patients and their risk of adverse outcomes. In addition, it is crucial that we find tools to accurately identify these patients. We hypothesised that the severity of disease present at the moment of the ED visit, is both a reflection of the premorbid condition and the current disease, and therefore, an estimation of the severity of disease may probably serve as a good predictor of clinical outcome in older patients.

The major aim of this thesis is, therefore, to find accurate and easy tools to identify patients at risk of adverse outcome. To do so, we aimed at identifying early predictors of adverse outcome (e.g. patient characteristics, vital signs, clinical intuition, routine laboratory tests and biomarkers). Furthermore, we aimed at developing and validating an easy to use prediction model for short-term mortality. Variables which were easily available and reliably retrievable during the short phase of ED stay were used. Finally, we aimed at identifying the different types of concerns of older ED patients and their caregivers and their association with adverse outcomes.

In this thesis, data of the RIsk Stratification in the Emergency department in acUtely ill older Patients (RISE UP) study were used. The RISE UP study is a prospective multicentre observational cohort study that aimed to identify predictors of adverse outcome in older medical ED patients. The study was conducted at the EDs of 
Zuyderland Medical Centre (MC) and Maastricht University Medical Centre (MUMC+) in the Netherlands. Patients who were 65 years or older, who visited one of the participating EDs between July 2016 until February 2017 and who were assessed and treated by internists or gastroenterologists, were eligible for inclusion. Patients were excluded when they were unable to speak Dutch, German or English or were admitted to another ward than internal medicine or gastroenterology. More information about the design, setting, population, objectives and procedures of the study can be found in Chapter 2.

Chapter 3 will describe the discriminatory value of clinical intuition (i.e. the disease perception and self-rated health of patients and the first clinical impression of nurses and physicians) with regard to short-term mortality and other adverse outcomes. Chapter 4 shows the different types of concerns of older ED patients and caregivers and the association of different concern categories with adverse outcomes. The development and validation of a new simplified prediction model for short-term mortality, the RISE UP score, is presented in Chapter 5. In Chapter 6, the discriminatory value of the biomarkers lactate, hs-cTnT, NT-proBNP, D-dimer and PCT for mortality and other adverse outcomes, and their added value to the RISE UP score, was evaluated. In Chapter 7 the incidence of MACE and 1-year mortality, combined with the discriminatory value of NT-proBNP and hs-cTnT for prediction of MACE in older ED patients who present with non-cardiac medical complaints, will be discussed.

\section{Pandemic}

During the process of this PhD trajectory, the world has been struck by a pandemic with COVID-19, caused by the severe acute respiratory syndrome coronavirus 2 (SARSCoV2). During the first wave of the pandemic, approximately $70 \%$ of the patients who were admitted were 65 years or older and most COVID-related deaths concerned older patients. This brought us to the idea to validate the newly designed RISE UP score in COVID-19 patients. In Chapter 8, the validation of the RISE UP score in ED patients with COVID-19 will be described. 


\section{References}

1. World Population Ageing 2019. New York: United Nations - Department of Economic and Social Affairs, 2020.

2. Prognose bevolking; geslacht en leeftijd, 2020-2060: Centraal Bureau voor Statistiek, 2019.

3. Monitor acute zorg 2018. Utrecht: Nederlandse Zorgautoriteit, 2018.

4. Gebruik acute zorg, gebruik SEH naar leeftijd: Volksgezondheidenzorg.info; 2016 [Available from: https://www.volksgezondheidenzorg.info/onderwerp/acute-zorg/cijfers-context/gebruik-acutezorg\#node-gebruik-seh-naar-leeftijd.

5. Goldstein J, McVey J, Ackroyd-Stolarz S. The Role of Emergency Medical Services in Geriatrics: Bridging the Gap between Primary and Acute Care. CJEM 2016;18(1):54-61.

6. Aminzadeh F, Dalziel WB. Older adults in the emergency department: a systematic review of patterns of use, adverse outcomes, and effectiveness of interventions. Ann Emerg Med 2002;39(3):238-47.

7. Salvi F, Morichi V, Grilli A, et al. The elderly in the emergency department: a critical review of problems and solutions. Intern Emerg Med 2007;2(4):292-301.

8. Wachelder JJH, Stassen PM, Hubens L, et al. Elderly emergency patients presenting with non-specific complaints: Characteristics and outcomes. PloS One 2017;12(11):e0188954.

9. Brouns SH, Stassen PM, Lambooij SL, et al. Organisational Factors Induce Prolonged Emergency Department Length of Stay in Elderly Patients--A Retrospective Cohort Study. PloS One 2015;10(8):e0135066.

10. Baraff $\mathrm{L}$, Bernstein $\mathrm{E}$, Bradley $\mathrm{K}$, et al. Perceptions of emergency care by the elderly: results of multicenter focus group interviews. Ann Emerg Med 1992;21(7):814-8.

11. Watson WT, Marshall ES, Fosbinder D. Elderly patients' perceptions of care in the emergency department. J Emerg Nurs 1999;25(2):88-92.

12. Kihlgren AL, Nilsson M, Skovdahl K, et al. Older patients awaiting emergency department treatment. Scand J Caring Sci 2004;18(2):169-76.

13. Samaras N, Chevalley T, Samaras D, et al. Older patients in the emergency department: a review. Ann Emerg Med 2010;56(3):261-9.

14. Buurman BM, de Rooij SE. Functional decline in older persons after acute hospitalization. Ned Tijdschr Geneeskd 2015;159:A8185.

15. Buurman BM, van Munster BC, Korevaar JC, et al. Prognostication in acutely admitted older patients by nurses and physicians. J Gen Intern Med 2008;23(11):1883-9.

16. Heron M. Deaths: Leading Causes for 2017. National vital statistics reports : from the Centers for Disease Control and Prevention, National Center for Health Statistics, National Vital Statistics System 2019;68(6):1-77.

17. Causes of death statistics - people over 65: Eurostat, 2016.

18. Carpenter CR, Shelton E, Fowler S, et al. Risk factors and screening instruments to predict adverse outcomes for undifferentiated older emergency department patients: a systematic review and metaanalysis. Acad Emerg Med 2015;22(1):1-21.

19. de Gelder J, Lucke JA, de Groot B, et al. Predicting adverse health outcomes in older emergency department patients: the APOP study. Neth J Med 2016;74(8):342-52.

20. McCusker J, Jacobs $\mathrm{P}$, Dendukuri $\mathrm{N}$, et al. Cost-effectiveness of a brief two-stage emergency department intervention for high-risk elders: results of a quasi-randomized controlled trial. Ann Emerg Med 2003;41(1):45-56.

21. Gill TM. The Central Role of Prognosis in Clinical Decision Making. JAMA 2012;307(2):199-200.

22. Yourman LC, Lee SJ, Schonberg MA, et al. Prognostic indices for older adults: a systematic review. JAMA 2012;307(2):182-92.

23. de Groot B, Stolwijk F, Warmerdam M, et al. The most commonly used disease severity scores are inappropriate for risk stratification of older emergency department sepsis patients: an observational multi-centre study. Scand J Trauma Resusc Emerg Med 2017;25(1):91.

24. Westendorp RGJ, Kusumastuti S. Screening tools do not measure up against rigorous history taking. Ned Tijdschr Geneeskd 2019;163 
25. Ellis G, Whitehead MA, Robinson D, et al. Comprehensive geriatric assessment for older adults admitted to hospital: meta-analysis of randomised controlled trials. BMJ (Clinical research ed) 2011;343:d6553.

26. Grossmann FF, Zumbrunn T, Ciprian S, et al. Undertriage in older emergency department patients-tilting against windmills? PloS One 2014;9(8):e106203.

27. Mackway-Jones K. Emergency triage. London: BMJ Publishing Group 19971997.

28. Zachariasse JM, Seiger N, Rood PPM, et al. Validity of the Manchester Triage System in emergency care: A prospective observational study. PloS One 2017;12(2):e0170811.

29. Steiner D, Renetseder F, Kutz A, et al. Performance of the Manchester Triage System in Adult Medical Emergency Patients: A Prospective Cohort Study. J Emerg Med 2016;50(4):678-89.

30. Charlson ME, Sax FL, MacKenzie CR, et al. Assessing illness severity: does clinical judgment work? J Chronic Dis 1986;39(6):439-52.

31. Brabrand M, Hallas J, Knudsen T. Nurses and physicians in a medical admission unit can accurately predict mortality of acutely admitted patients: a prospective cohort study. PloS One 2014;9(7):e101739.

32. Beglinger B, Rohacek M, Ackermann S, et al. Physician's first clinical impression of emergency department patients with nonspecific complaints is associated with morbidity and mortality. Medicine (Baltimore) 2015;94(7):e374.

33. van der Vegt $A E$, Holman $M$, ter Maaten JC. The value of the clinical impression in recognizing and treating sepsis patients in the emergency department. Eur J Emerg Med 2012;19(6):373-8.

34. Rohacek $\mathrm{M}$, Nickel $\mathrm{CH}$, Dietrich $\mathrm{M}$, et al. Clinical intuition ratings are associated with morbidity and hospitalisation. Int J Clin Pract 2015;69(6):710-7.

35. Wong DD, Wong RP, Caplan GA. Self-rated health in the unwell elderly presenting to the emergency department. Emerg Med Aust 2007;19(3):196-202.

36. Godard-Sebillotte $\mathrm{C}$, Dramé M, Basileu T, et al. Is self-rated health an independent prognostic factor of six-week mortality in older patients hospitalized for an acute condition? Qual Life Res 2016;25(9):2335-40.

37. Downar J, Goldman R, Pinto R, et al. The "surprise question" for predicting death in seriously ill patients: a systematic review and meta-analysis. CMAJ 2017;189(13):E484-e93.

38. White $M$, Edmondson $D$, Umland $R$, et al. Patient perceptions of stress during evaluation for ACS in the ED. Am J Emerg Med 2017;35(2):351-52.

39. Froom $\mathrm{P}$, Shimoni Z. Prediction of hospital mortality rates by admission laboratory tests. Clin Chem 2006;52(2):325-8.

40. Loekito E, Bailey J, Bellomo R, et al. Common laboratory tests predict imminent death in ward patients. Resuscitation 2013;84(3):280-5.

41. de Gelder J, Lucke JA, Heim N, et al. Predicting mortality in acutely hospitalized older patients: a retrospective cohort study. Intern Emerg Med 2016;11(4):587-94.

42. Barfod C, Lundstrom LH, Lauritzen MM, et al. Peripheral venous lactate at admission is associated with in-hospital mortality, a prospective cohort study. Acta Anaesth Scand 2015;59(4):514-23.

43. Datta D, Walker C, Gray AJ, et al. Arterial lactate levels in an emergency department are associated with mortality: a prospective observational cohort study. Emerg Med J 2015;32(9):673-7.

44. Pedersen M, Brandt VS, Holler JG, et al. Lactate level, aetiology and mortality of adult patients in an emergency department: a cohort study. Emerg Med J 2015;32(9):678-84.

45. van den Nouland DP, Brouwers MC, Stassen PM. Prognostic value of plasma lactate levels in a retrospective cohort presenting at a university hospital emergency department. BMJ Open 2017;7(1):e011450.

46. Bahrmann P, Christ M, Hofner B, et al. Prognostic value of different biomarkers for cardiovascular death in unselected older patients in the emergency department. Eur Heart J Acute Cardiovasc Care 2016;5(8):568-78.

47. de Groot B, Verdoorn RC, Lameijer J, et al. High-sensitivity cardiac troponin T is an independent predictor of inhospital mortality in emergency department patients with suspected infection: a prospective observational derivation study. Emerg Med J 2014;31(11):882-8. 
48. Courtney D, Conway R, Kavanagh J, et al. High-sensitivity troponin as an outcome predictor in acute medical admissions. Postgrad Med J 2014;90(1064):311-6.

49. Nickel $\mathrm{CH}$, Kuster T, Keil C, et al. Risk stratification using D-dimers in patients presenting to the emergency department with nonspecific complaints. Eur J Intern Med 2016;31:20-4

50. Luchner A, Mockel M, Spanuth E, et al. N-terminal pro brain natriuretic peptide in the management of patients in the medical emergency department (PROMPT): correlation with disease severity, utilization of hospital resources, and prognosis in a large, prospective, randomized multicentre trial. Eur J Heart Fail 2012;14(3):259-67.

51. Rodelo JR, De la Rosa G, Valencia ML, et al. D-dimer is a significant prognostic factor in patients with suspected infection and sepsis. Am J Emerg Med 2012;30(9):1991-9.

52. Wilhelm J, Hettwer S, Hammer D, et al. Outcome prediction using clinical scores and biomarkers in patients with presumed severe infection in the emergency department. Med Klin Intensivmed Notfallmed 2012;107(7):558-63.

53. Freund $\mathrm{Y}$, Delerme $\mathrm{S}$, Goulet $\mathrm{H}$, et al. Serum lactate and procalcitonin measurements in emergency room for the diagnosis and risk-stratification of patients with suspected infection. Biomarkers 2012;17(7):590-6.

54. Knaus WA, Draper EA, Wagner DP, et al. APACHE II: a severity of disease classification system. Crit Care Med 1985;13(10):818-29.

55. Subbe $C P$, Kruger $M$, Rutherford $P$, et al. Validation of a modified Early Warning Score in medical admissions. QJM 2001;94(10):521-6.

56. McCusker J, Bellavance F, Cardin S, et al. Detection of older people at increased risk of adverse health outcomes after an emergency visit: the ISAR screening tool. J Am Geriatr Soc 1999;47(10):1229-37.

57. Hoogerduijn JG, Buurman BM, Korevaar JC, et al. The prediction of functional decline in older hospitalised patients. Age Ageing 2012;41(3):381-7.

58. de Gelder J, Haenen E, Lucke JA, et al. Optimising the ISAR-HP to screen efficiently for functional decline in older patients. Neth J Med 2017;75(9):379-85

59. Yao JL, Fang J, Lou QQ, et al. A systematic review of the identification of seniors at risk (ISAR) tool for the prediction of adverse outcome in elderly patients seen in the emergency department. Int J Clin Exp Med 2015;8(4):4778-86

60. Wallis SJ, Wall J, Biram RW, et al. Association of the clinical frailty scale with hospital outcomes. QJM 2015;108(12):943-9.

61. Jørgensen R, Brabrand M. Screening of the frail patient in the emergency department: A systematic review. Eur J Intern Med 2017;45:71-73.

62. Kaeppeli T, Rueegg M, Dreher-Hummel T, et al. Validation of the Clinical Frailty Scale for Prediction of Thirty-Day Mortality in the Emergency Department. Ann Emerg Med 2020

63. Hewitt J, Carter B, Vilches-Moraga A, et al. The effect of frailty on survival in patients with COVID-19 (COPE): a multicentre, European, observational cohort study. Lancet Public Health 2020

64. Elliott A, Phelps $K$, Regen $E$, et al. Identifying frailty in the Emergency Department-feasibility study. Age Ageing 2017;46(5):840-45.

65. van Dam CS, Trappenburg MC, Peters MJL, et al. Screening for vulnerability in older adults attending the emergency department. Ned Tijdschr Geneeskd 2019;163. 


\section{Chapter 2}

Study protocol for a multicentre prospective cohort study to identify predictors of adverse outcome in older medical emergency department patients (the Risk Stratification in the Emergency Department in Acutely III Older Patients (RISE UP) study)

N. Zelis, J. Buijs, P.W. de Leeuw, S.M.J. van Kuijk,

P.M. Stassen 


\section{Abstract}

\section{Background}

Older patients ( $\geq 65$ years old) experience high rates of adverse outcomes after an emergency department (ED) visit. Reliable tools to predict adverse outcomes in this population are lacking. This manuscript comprises a study protocol for the Risk Stratification in the Emergency Department in Acutely III Older Patients (RISE UP) study that aims to identify predictors of adverse outcome (including triage- and risk stratification scores) and intends to design a feasible prediction model for older patients that can be used in the ED.

\section{Methods}

The RISE UP study is a prospective observational multicentre cohort study in older ( $\geq 65$ years of age) ED patients treated by internists or gastroenterologists in Zuyderland Medical Centre and Maastricht University Medical Centre+ in the Netherlands.

After obtaining informed consent, patients characteristics, vital signs, functional status and routine laboratory tests will be retrieved. In addition, disease perception questionnaires will be filled out by patients or their caregivers and clinical impression questionnaires by nurses and physicians. Moreover, both arterial and venous blood samples will be taken in order to determine additional biomarkers. The discriminatory value of triage- and risk stratification scores, clinical impression scores and laboratory tests will be evaluated.

Univariable logistic regression will be used to identify predictors of adverse outcomes. With these data we intend to develop a clinical prediction model for 30-day mortality using multivariable logistic regression. This model will be validated in an external cohort.

Our primary endpoint is 30-day all-cause mortality. The secondary (composite) endpoint consist of length of hospital stay, admission to intensive- or medium care units, readmission and loss of independent living.

Patients will be followed for at least 30 days and, if possible, for one year.

\section{Discussion}

In this study, we will retrieve a broad range of data concerning adverse outcomes in older patients visiting the ED with medical problems. We intend to develop a clinical tool for identification of older patients at risk of adverse outcomes that is feasible for use in the ED, in order to improve clinical decision making and medical care. 


\section{Background}

Older patients ( $\geq 65$ years of age) constitute an increasing population in emergency departments (EDs). They experience more adverse outcomes than younger ED patients $^{1-3}$ as their ED visits are often highly urgent and followed by hospitalization, Intensive Care Unit (ICU) admission, readmission, and mortality (up to 10\% within 3 months). In older ED patients with internal medicine (medical) problems, mortality is even higher (23.8\% in 3 months). ${ }^{4}$

Risk stratification can be used to identify ED patients who are at high risk of adverse outcomes. This may lead to improvement in medical care and outcome by early start of interventions. ${ }^{5}$ Several risk stratification scores have been developed for the older ED population, such as the Identification of Seniors at Risk (ISAR) ${ }^{6}$ or Triage Risk Stratification Tool (TRST). ${ }^{7}$ Unfortunately, these scores do not accurately identify those who experience adverse outcomes (areas under the curve (AUCs) range from $0.59-0.74) .^{8-10}$ Triage systems (e.g., Manchester Triage System (MTS) ${ }^{11}$ ) are also used in the ED population but they tend to undertriage older patients. ${ }^{12}$ Furthermore, risk stratification scores either applicable to the general ED population (e.g., Acute Physiology and Chronic Health Evaluation II (APACHE II) score ${ }^{13}$ ) or to patients with specific diseases (e.g. Abbreviated Mortality ED Sepsis (abbMEDS) score ${ }^{14}$ for sepsis) are used. However, these are not validated in the older ED population.

It may be possible that older patients at risk of adverse outcomes can be identified by assessing the disease perception of patients (or caregivers) or clinical intuition or impression of physicians and/or nurses. Indeed, both disease perception and clinical impression are shown to be associated with mortality and morbidity. ${ }^{15-20}$ Unfortunately, most studies were conducted in other clinical settings than the ED (e.g., admission units and ICUs) ${ }^{4,17,20}$ and in younger patients. ${ }^{17,18,20} \mathrm{~A}$ second method to assess clinical impression is to ask the 'surprise question' (SQ): 'Would I be surprised if this patient died within the next 12 months?'. The SQ has been studied in cancer and renal failure patients but its diagnostic accuracy for one-year mortality varies considerably. ${ }^{21}$ The predictive value of the SQ for short-term mortality in older medical ED patients is unknown.

Laboratory tests may also be useful for identification of older patients at risk of adverse outcomes. ${ }^{4,22}$ In addition, non-routine laboratory tests, such as lactate, highsensitivity cardiac troponin $\mathrm{T}$ (hs-cTnT), $\mathrm{N}$-terminal pro-B-type natriuretic peptide (NT-pro-BNP), procalcitonin (PCT) and d-dimer, may be valuable predictors of adverse 
outcome as well. ${ }^{23-27}$ Since these tests are indicators of serious conditions and diseases (e.g. tissue hypoperfusion, myocardial injury, bacterial infection and thromboembolism) and often present in the older ED population, we hypothesize that these tests can be useful as predictors. Until now, most studies regarding the predictive value of these tests were performed retrospectively ${ }^{24,28-33}$ or in selected, mostly younger, patients. ${ }^{30-38}$

We hypothesize that in the early stage of an ED visit, when important treatment decisions have to be made, several factors can predict adverse outcomes. The aims of this multicentre, prospective study are to 1) identify early predictors of adverse outcome in older ED patients, and 2) develop a clinical prediction model for 30-day mortality.

\section{Methods and design}

\section{Study design and setting}

This prospective multicentre observational cohort study will take place at the EDs of Zuyderland Medical Centre (MC) Heerlen and Maastricht University Medical Centre+ (MUMC+), in the Netherlands. Zuyderland MC is a secondary teaching hospital with 635 beds and 30,000 ED visits/year. MUMC+ is a secondary and tertiary teaching hospital with 700 beds and approximately 23,000 ED visits/year.

\section{Study population}

All patients $\geq 65$ years of age, assessed and treated by an internist or gastroenterologist at the EDs during the study period, are eligible for study inclusion. We chose medical ED patients because these patients are at high risk of adverse outcomes after an ED visit [4]. We intend to include 450 patients in Zuyderland MC starting from July 2016, and 150 patients in MUMC+ from September 2016.

Inclusion criteria:

- Age $\geq 65$ years

- Treatment by an internist, gastroenterologist (or emergency physician under supervision of an internist/gastroenterologist)

- Informed consent 
Exclusion criteria:

- Earlier participation in this study

- No informed consent

- Inability to speak Dutch or English

- Admission to a ward of another specialty than internal medicine/ gastroenterology

In case a patient is unable to provide informed consent, e.g. in case of delirium, dementia or when a patient is too severely ill to answer the questions, a legal representative can provide informed consent. A legal representative can either be a legal guardian or an immediate family member including their spouse, adult children, parents or adult siblings. The determination, whether or not a patient can provide informed consent for themselves, will be based on expect opinion by the attending physician or investigator. Objection by an incapacitated patient or his/her representatives will always lead to exclusion from the study and analysis.

\section{Objectives and Outcome}

\section{Objectives}

1. Evaluation of the discriminatory value for adverse outcomes of:

a. triage and risk stratification scores:

- Triage score: MTS [11]

- General risk stratification scores: APACHE $\|^{13}$, ISAR score ${ }^{6}$ and ISARHospitalised Patients (ISAR-HP) score ${ }^{39}$

- Disease specific scores: Glasgow-Blatchford Bleeding Score (GBS) ${ }^{40}$, abbMEDS score $^{14}$, Sepsis-related Organ Failure Assessment (SOFA) score $^{41}$ and Confusion, Urea, Respiration, Blood pressure, Age $>65$ years (CURB-65) score ${ }^{42}$

b. clinical impressions of nurses and physicians, SQ and disease perception of patients

c. routine and non-routine laboratory results

2. Development and validation of a prediction model for short-term mortality and test the predictive ability of this model for the other adverse outcomes/endpoints

\section{Outcomes}

Primary endpoint:

- 30-day (both in- and out-hospital) all-cause mortality 
Secondary endpoints:

a. Secondary composite endpoint:

- Length of hospital stay (LOS) $>7$ days

- Intensive or medium care unit (ICU/MCU) admission

- Unplanned readmission within 30 days after discharge

- Loss of independent living (e.g. discharge to a nursing home/hospice or with palliative care in previously community dwelling patients)

b. 1-year all-cause mortality

\section{Study Procedures}

\section{Inclusion of patients}

After arrival at the ED, all eligible patients will receive an information brochure and will be asked to participate in the study by the attending physician or investigator. Informed consent must be signed by the patient or his/her legal representative before entering the study. Figure 2.1 details the study procedure.

\section{Questionnaires}

The patient/caregiver will receive a questionnaire at the ED that should be filled out as soon as possible. This questionnaire (Supplemental data, Questionnaire 1) contains four questions regarding disease and health perception. The nurse and attending physician both receive a similar questionnaire (Supplemental data, Questionnaire 2 and 3) that has to be filled out before history taking and physical examination and without knowing any diagnostic results. This questionnaire contains six questions regarding the first clinical impression including the SQ. When a patient is admitted to the hospital, a fourth questionnaire (Supplemental data, Questionnaire 4), containing ten questions about the patients' daily functioning, will be filled out the next day. This questionnaire is used to calculate the ISAR-HP and Katz Activities of Daily Living $(A D L)^{43}$ index score. The results of the questionnaires will only be available for the investigators.

\section{Blood sample collection}

In addition to routine blood sampling, two venous blood samples and an arterial blood gas sample will be collected at the ED. Venous blood samples will be stored in a freezer at -20 degrees Celsius and will be analysed for hs-cTnT, NT-pro-BNP, PCT and d-dimer after 4-12 weeks. Results will be blinded for the physician. Results of the arterial blood gas and lactate level analysis will be presented to the attending 
physician. Additional venous and arterial blood sample collections will only be performed in Zuyderland MC.

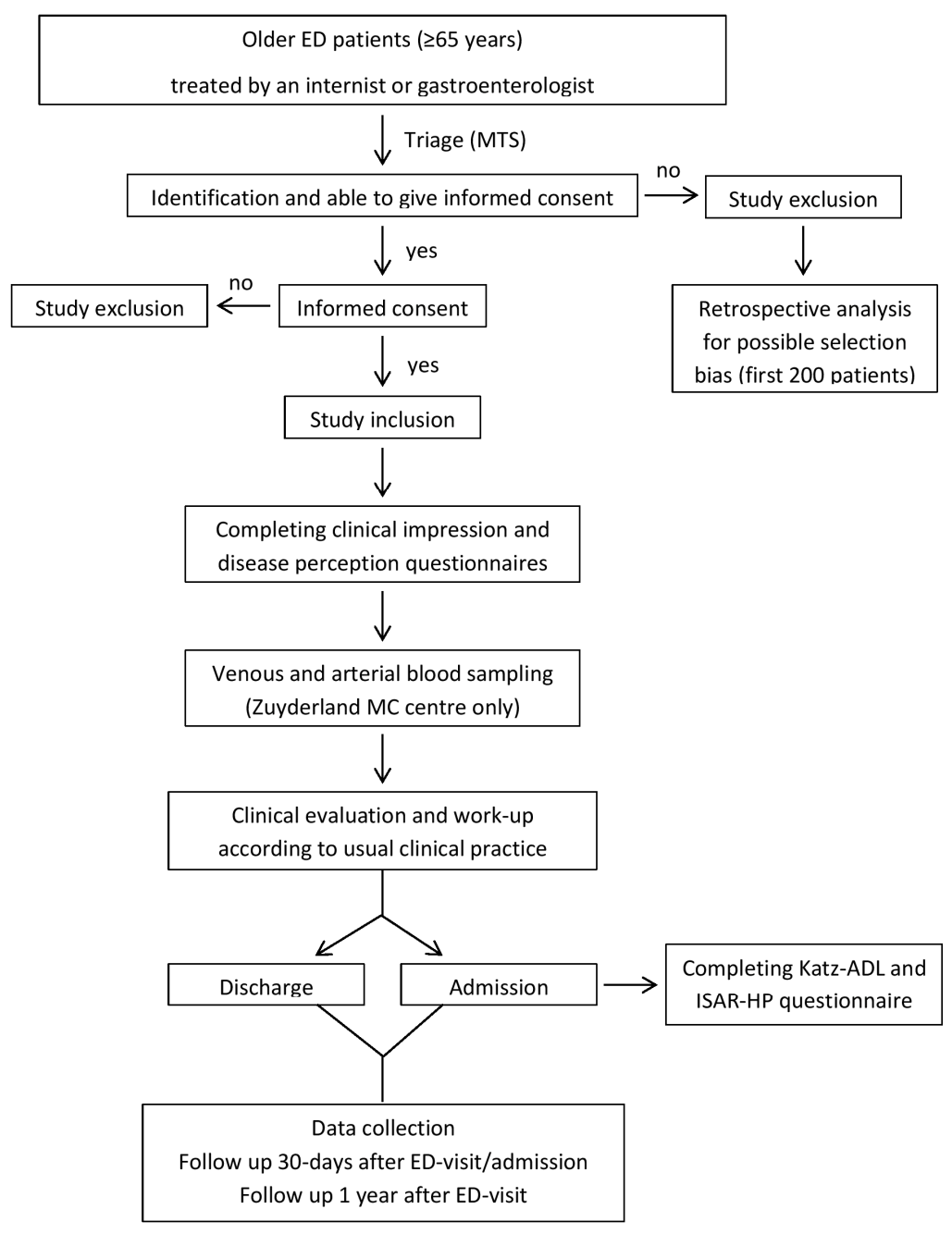

Figure 2.1 Flow chart of study procedure

\section{Data collection and follow up}

Study parameters will be retrieved from the patient's medical electronic record and questionnaires. All patients will be followed up for 1 year to obtain long-term outcomes. The following parameters will be collected: 
Study parameters collected at the ED

- Demographics (age, sex)

- Date and time of ED visit and transport to the ED

- Comorbidities: Charlson Comorbidity Index ${ }^{44}$, smoking status and presence of cardiovascular disease in family history

- Vital signs: heart rate, blood pressure, respiratory rate, oxygen saturation, temperature, Glasgow Coma Scale ${ }^{45}$

- First clinical impression (including the SQ) of the physician/nurse and disease perception of the patient/caregiver using questionnaires

- Cognitive functioning (dementia, mild cognitive impairment, delirium or normal) based on the diagnosis of a geriatrician and/or on medical records

- Number of visits to the hospital in the preceding year

- Medication use before the ED visit

- Time spent at the ED and the number of physician consultations and radiological examinations during ED stay

- Routine laboratory tests: glucose, creatinine, blood urea nitrogen, sodium, potassium, chloride, bicarbonate, calcium, phosphate, bilirubin, alkaline phosphatase, gamma-glutamyltransferase, aspartate transaminase, alanine transaminase, lactate dehydrogenase, albumin, c-reactive protein, hemoglobin, hematocrit, leukocyte count, platelet count, international normalized ratio and activated partial thromboplastin time

- Non-routine laboratory tests: arterial blood gas, lactate, hs-cTnT, NT-pro-BNP, PCT and d-dimer

- Triage score: MTS

- General risk stratification scores:

\section{APACHE II score}

2. ISAR score

- Disease specific stratification scores:

1. GBS for upper gastrointestinal bleeding

2. abbMEDS score for sepsis

3. SOFA score for sepsis

4. CURB-65 score for pneumonia

These scores were only calculated when the specific disease for which the score was developed was present.

- Diagnosis at the time of discharge from the ED 
Study parameters collected in admitted patients only

- Functional capability:

1. Katz ADL index score

2. ISAR-HP score

- Diagnosis at time of discharge from the hospital

- $\quad$ LOS (days)

- $\quad$ Living arrangement after discharge: e.g. community dwelling, nursing- or care home etc.

Follow up study parameters collected in all participants:

- ICU/MCU admission

- All-cause mortality within 30 days of the ED visit

- Readmission within 30 days after discharge

- Any new relevant medical condition within 1 year after ED visit (e.g. new diagnose of venous thromboembolism, cardiovascular disease or malignancy)

\section{Possible selection bias}

Because physicians must give priority to providing emergency care when the ED is crowded, we expect that not all possible candidates can be included. To investigate possible selection bias, we will retrospectively form a sample of non-included patients and collect the same data, except for the non-routine tests and questionnaires, as in our prospective cohort population. For practical reasons, we intend to include the first 200 non-included candidates in this retrospective sample. Patients who refused to participate in the study will not be included. Baseline characteristics (age and sex) will be analysed for all (non-included) candidates to investigate possible selection bias as well.

\section{Study analysis}

\section{Statistical analysis}

First, patients characteristics and outcomes will be described. Continuous variables will be reported as means with standard deviations or medians with interquartile range and categorical variables as proportions. Valid percentages will be used when values are missing.

Secondly, we will quantify the ability of the risk-stratification scores, clinical impression scores and non-routine laboratory tests to discriminate between the presence and absence of the different endpoints separately using the area under the 
receiver operator characteristics curve (AUC-ROC). We will determine their diagnostic accuracy using sensitivity, specificity, positive and negative predictive values (PPV, NPV), likelihood ratios and Youden's index. For the discriminatory value of riskstratification scores and clinical impression scores, combined data from both centres will be used. For the non-routine laboratory tests, only data from patients recruited in Zuyderland MC will be used.

Third, we will identify possible predictors of adverse outcome using univariable logistic regression analyses. Odds ratios (OR) with $95 \%$ confidence intervals $(95 \% \mathrm{Cl})$ will be calculated. Continuous variables will be checked for nonlinearity and collinearity. In case of missing items, we will use stochastic regression imputation to impute these items using predictive mean matching.

\section{Model development}

We will develop a clinical prediction model for 30-day mortality using multivariable logistic regression with predictors that are deemed feasible. We consider predictors feasible when a parameter is available in at least $90 \%$ of the participants at the ED, reproducible and easily retrieved. Participants that are prospectively included in Zuyderland MC and MUMC+ will form the derivation cohort and their data will be used for model development. For external validation, we will retrospectively collect data of ED patients to form a validation cohort. For the development and validation of our model the Stiell criteria will be applied. ${ }^{46}$

After external validation, we will test the predictive ability of the model for the secondary composite endpoint and test whether addition of clinical impression scores and/or non-routine laboratory tests results in a better prediction of adverse outcomes.

All data will be analysed using IBM SPSS Statistics for Windows, Version 24.0 (IBM Corp., Armonk, N.Y., USA) and R version 3.3.3.

\section{Sample size calculation}

Since we will be using logistic regression analysis to identify predictors of 30-day mortality, a minimum of 10 events per candidate predictor is suggested. ${ }^{47}$ Expected 30 -day mortality in older medical ED patients is assumed to be at least $10 \%$ based on previous studies in the Netherlands. ${ }^{4,48}$ Therefore, we decided to include 450 patients in Zuyderland MC and 150 patients in MUMC+ to form the derivation cohort. For external validation of our prediction model, we need approximately 100 events, and therefore, the sample size of the validation cohort will be based on the mortality rate of the derivation cohort (estimation: 800 patients). 


\section{Trial status}

As of $22 / 12 / 2018$, the study is still ongoing; we are about to finish the inclusion of patients for the validation cohort and are completing 1 year follow up. A total of 603 participants are included in the derivation cohort from July 2016 until the beginning of February 2017 in the two participating centres. In Zuyderland MC, 450 patients, and in MUMC+, 153 patients are included.

\section{Discussion}

Early risk stratification at the ED is extremely important to optimise treatment and improve outcomes in acutely ill older patients. To identify older patients with increased risk of adverse outcomes in an early stage, we need accurate predictors.

In the past decades several studies identified risk factors and predictors of adverse outcomes in the older ED population. ${ }^{4,8,49}$ However, most of these studies were conducted in an unselected population of older patients, leading to conflicting results. For our study we chose medical ED patients because we assume that this group of patients represent a large group of patients who are highly at risk of adverse outcomes. Furthermore, we chose to evaluate more endpoints (including composite endpoints) because some adverse outcomes exclude others (i.e. dying in-hospital will prevent readmission). We are convinced that for quantification of the risk of adverse outcomes in the older population, more than one endpoint is needed for a reliable clinical appraisal. We consider patients $\geq 65$ years to be old and we chose this cut off based on other well-known screening instruments such as the ISAR ${ }^{6}$, the ISAR-HP ${ }^{39}$ and TRST. ${ }^{7}$

Development and validation of our prediction model for 30-day mortality will be according to the Stiell criteria. ${ }^{46}$ Since reliable tools to predict short-term mortality are lacking there is need for such a prediction model (criterion 1). Before conduct of this study, internists, gastroenterologists and geriatricians were consulted on the need of a clinical tool, preferred outcomes to be studied and potentially meaningful predictors. The model will be derived according to methodologic standards and is intended to be easily implemented in routine ED care (criterion 2). Most of the predictors we will select will resemble the morbid state rather that the premorbid state because we hypothesize that that the severity of the disease for which the patient visits the ED (morbid state) may be more important than the premorbid state 
for prediction of short-term mortality. We aim to externally validate our prediction model in a different ED population (criterion 3). Once we have succeeded in developing and externally validating an accurate model, we intend to implement it into clinical practice by offering an online calculator, which may also be incorporated into a electronical file management system (criterion 4).

The main strengths of the RISE UP study are, in our opinion, its prospective multicentre study design in an ED setting and the use of composite endpoints. We aim to identify predictors of adverse outcomes in an early stage of presentation, in the ED setting, when important decisions need to be made. We will not only evaluate the predictive value of the clinical impression of both the patient, nurse and physician, but also that of routine and non-routine laboratory tests. A possible limitation is that we expect that we cannot include all potential candidates, due to crowding of patients at the ED or other logistic problems. Therefore, we will perform an additional analysis to investigate possible selection bias. In addition, this study will include internal medicine and gastroenterology patients only. If this study yields an accurate model, that model will have to be tested in the overall older ED population. Furthermore, we intend to implement the model into clinical practice by use of an online calculator. Possibly, this may also be incorporated into an electronical file management system or a mobile App.

In summary, the RISE UP study is a prospective multicentre cohort study that aims to identify predictors of adverse outcomes in older medical ED patients. The goal of this study is to develop a practical, feasible tool to identify older ED patients with an increased risk of adverse outcomes in an early stage, in order to improve their care in the future.

\section{Acknowledgements}

We are grateful for the funding from Zuyderland $M C$ and we would like to thank all of the patients, nurses and physicians who are part of the study. 


\section{References}

1. Aminzadeh F, Dalziel WB. Older adults in the emergency department: a systematic review of patterns of use, adverse outcomes, and effectiveness of interventions. Ann Emerg Med. 2002;39(3):238-247.

2. Samaras N, Chevalley T, Samaras D, Gold G: Older patients in the emergency department: a review. Ann Emerg Med. 2010;56(3):261-269.

3. Salvi F, Morichi V, Grilli A, Giorgi R, De Tommaso G, Dessi-Fulgheri P. The elderly in the emergency department: a critical review of problems and solutions. Intern Emerg Med. 2007;2(4):292-301.

4. Buurman BM, van Munster BC, Korevaar JC, Abu-Hanna A, Levi M, de Rooij SE. Prognostication in acutely admitted older patients by nurses and physicians. J Gen Intern Med. 2008;23(11):1883-1889.

5. McCusker J, Jacobs P, Dendukuri N, Latimer E, Tousignant P, Verdon J. Cost-effectiveness of a brief two-stage emergency department intervention for high-risk elders: results of a quasi-randomized controlled trial. Ann Emerg Med. 2003;41(1):45-56.

6. McCusker J, Bellavance F, Cardin S, Trepanier S, Verdon J, Ardman O. Detection of older people at increased risk of adverse health outcomes after an emergency visit: the ISAR screening tool. J Am Geriatr Soc. 1999;47(10):1229-1237.

7. Meldon SW, Mion LC, Palmer RM, Drew BL, Connor JT, Lewicki LJ, Bass DM, Emerman CL. A brief riskstratification tool to predict repeat emergency department visits and hospitalizations in older patients discharged from the emergency department. Acad Emerg Med. 2003;10(3):224-232.

8. Carpenter CR, Shelton E, Fowler S, Suffoletto B, Platts-Mills TF, Rothman RE, Hogan TM. Risk factors and screening instruments to predict adverse outcomes for undifferentiated older emergency department patients: a systematic review and meta-analysis. Acad Emerg Med. 2015;22(1):1-21.

9. Yao JL, Fang J, Lou QQ, Anderson RM. A systematic review of the identification of seniors at risk (ISAR) tool for the prediction of adverse outcome in elderly patients seen in the emergency department. Int J Clin Exp Med. 2015;8(4):4778-4786.

10. Cousins G, Bennett Z, Dillon G, Smith SM, Galvin R. Adverse outcomes in older adults attending emergency department: systematic review and meta-analysis of the Triage Risk Stratification Tool. Eur J Emerg Med. 2013;20(4):230-239.

11. Mackway-Jones K, Group MT. Emergency triage. London: BMJ Publishing Group; 1997.

12. Grossmann FF, Zumbrunn T, Ciprian S, Stephan FP, Woy N, Bingisser R, Nickel CH. Undertriage in older emergency department patients--tilting against windmills? PloS One 2014;9(8):e106203.

13. Knaus WA, Draper EA, Wagner DP, Zimmerman JE. APACHE II: a severity of disease classification system. Crit Care Med. 1985;13(10):818-829.

14. Vorwerk C, Loryman B, Coats TJ, Stephenson JA, Gray LD, Reddy G, Florence L, Butler N. Prediction of mortality in adult emergency department patients with sepsis. Emerg Med J. 2009;26(4):254-258.

15. Charlson ME, Sax FL, Mackenzie CR, Fields SD, Braham RL, Douglas RG, Jr.. Assessing illness severity: does clinical judgment work? J Chronic Dis. 1986;39(6):439-452.

16. Charlson ME, Sax FL, MacKenzie CR, Braham RL, Fields SD, Douglas RG, Jr.. Morbidity during hospitalization: can we predict it? J Chronic Dis. 1987;40(7):705-712.

17. Brabrand M, Hallas J, Knudsen T. Nurses and physicians in a medical admission unit can accurately predict mortality of acutely admitted patients: a prospective cohort study. PloS One 2014;9(7): e101739.

18. Rohacek $\mathrm{M}$, Nickel $\mathrm{CH}$, Dietrich $\mathrm{M}$, Bingisser R. Clinical intuition ratings are associated with morbidity and hospitalisation. Int J Clin Pract. 2015;69(6):710-717.

19. Beglinger B, Rohacek M, Ackermann S, Hertwig R, Karakoumis-Ilsemann J, Boutellier S, Geigy N, Nickel C, Bingisser R. Physician's first clinical impression of emergency department patients with nonspecific complaints is associated with morbidity and mortality. Medicine 2015;94(7):e374.

20. Sinuff T, Adhikari NK, Cook DJ, Schunemann HJ, Griffith LE, Rocker G, Walter SD. Mortality predictions in the intensive care unit: comparing physicians with scoring systems. Crit Care Med. 2006;34(3): 878-885.

21. Downar J, Goldman R, Pinto R, Englesakis M, Adhikari NK. The "surprise question" for predicting death in seriously ill patients: a systematic review and meta-analysis. CMAJ. 2017, 189(13):E484-e493. 
22. de Gelder J, Lucke JA, Heim N, de Craen AJ, Lourens SD, Steyerberg EW, de Groot B, Fogteloo AJ, Blauw GJ, Mooijaart SP. Predicting mortality in acutely hospitalized older patients: a retrospective cohort study. Intern Emerg Med. 2016;11(4):587-594.

23. Datta D, Walker C, Gray AJ, Graham C. Arterial lactate levels in an emergency department are associated with mortality: a prospective observational cohort study. Emerg Med J. 2015;32(9): 673-677.

24. Courtney D, Conway R, Kavanagh J, O'Riordan D, Silke B. High-sensitivity troponin as an outcome predictor in acute medical admissions. Postgrad Med J. 2014;90(1064):311-316.

25. Luchner A, Mockel M, Spanuth E, Mocks J, Peetz D, Baum H, Spes C, Wrede CE, Vollert J, Muller R et al. $\mathrm{N}$-terminal pro brain natriuretic peptide in the management of patients in the medical emergency department (PROMPT): correlation with disease severity, utilization of hospital resources, and prognosis in a large, prospective, randomized multicentre trial. Eur J Heart Fail. 2012;14(3):259-267.

26. Hausfater P, Juillien G, Madonna-Py B, Haroche J, Bernard M, Riou B. Serum procalcitonin measurement as diagnostic and prognostic marker in febrile adult patients presenting to the emergency department. Crit Care (London, England) 2007;11(3):R60.

27. Nickel $\mathrm{CH}$, Kuster T, Keil C, Messmer AS, Geigy N, Bingisser R. Risk stratification using D-dimers in patients presenting to the emergency department with nonspecific complaints. Eur J Intern Med. 2016;31:20-24.

28. Pedersen M, Brandt VS, Holler JG, Lassen AT. Lactate level, aetiology and mortality of adult patients in an emergency department: a cohort study. Emerg Med J. 2015;32(9):678-684.

29. van den Nouland DP, Brouwers MC, Stassen PM. Prognostic value of plasma lactate levels in a retrospective cohort presenting at a university hospital emergency department. BMJ Open. 2017; 7(1):e011450.

30. Filho RR, Rocha LL, Correa TD, Pessoa CM, Colombo G, Assuncao MS. Blood Lactate Levels Cutoff and Mortality Prediction in Sepsis-Time for a Reappraisal? a Retrospective Cohort Study. Shock (Augusta, Ga) 2016;46(5):480-485.

31. Marchetti M, Benedetti A, Mimoz O, Lardeur JY, Guenezan J, Marjanovic N. Predictors of 30-day mortality in patients admitted to ED for acute heart failure. Am J Emerg Med. 2017;35(3):444-447.

32. Zhang $X H$, Dong $Y$, Chen $Y D$, Zhou $P$, Wang JD, Wen FQ. Serum N-terminal pro-brain natriuretic peptide level is a significant prognostic factor in patients with severe sepsis among Southwest Chinese population. Eur Rev Med Pharmacol Sci. 2013;17(4):517-521.

33. Mehta C, Dara B, Mehta Y, Tariq AM, Joby GV, Singh MK. Retrospective study on prognostic importance of serum procalcitonin and amino-terminal pro-brain natriuretic peptide levels as compared to Acute Physiology and Chronic Health Evaluation IV Score on Intensive Care Unit admission, in a mixed Intensive Care Unit population. Ann Card Anaesth. 2016;19(2):256-262.

34. Shapiro NI, Howell MD, Talmor D, Nathanson LA, Lisbon A, Wolfe RE, Weiss JW. Serum lactate as a predictor of mortality in emergency department patients with infection. Ann Emerg Med. 2005; 45(5):524-528.

35. Paneesha S, Cheyne E, French K, Bacchu S, Borg A, Rose P. High D-dimer levels at presentation in patients with venous thromboembolism is a marker of adverse clinical outcomes. $\mathrm{Br} \mathrm{J}$ Haematol. 2006, 135(1):85-90.

36. Knowlson L, Bacchu S, Paneesha S, McManus A, Randall K, Rose P. Elevated D-dimers are also a marker of underlying malignancy and increased mortality in the absence of venous thromboembolism. J Clin Pathol. 2010;63(9):818-822.

37. de Groot B, Verdoorn RC, Lameijer J, van der Velden J. High-sensitivity cardiac troponin $\mathrm{T}$ is an independent predictor of inhospital mortality in emergency department patients with suspected infection: a prospective observational derivation study. Emerg Med J. 2014;31(11):882-888.

38. Schuetz $P$, Birkhahn R, Sherwin R, Jones AE, Singer A, Kline JA, Runyon MS, Self WH, Courtney DM, Nowak RM et al. Serial Procalcitonin Predicts Mortality in Severe Sepsis Patients: Results From the Multicenter Procalcitonin MOnitoring SEpsis (MOSES) Study. Crit Care Med. 2017;45(5):781-789.

39. Hoogerduijn JG, Buurman BM, Korevaar JC, Grobbee DE, de Rooij SE, Schuurmans MJ. The prediction of functional decline in older hospitalised patients. Age Ageing. 2012;41(3):381-387. 
40. Blatchford O, Murray WR, Blatchford M. A risk score to predict need for treatment for uppergastrointestinal haemorrhage. Lancet (London, England) 2000;356(9238):1318-1321.

41. Vincent JL, Moreno R, Takala J, Willatts S, De Mendonca A, Bruining H, Reinhart CK, Suter PM, Thijs LG. The SOFA (Sepsis-related Organ Failure Assessment) score to describe organ dysfunction/failure. On behalf of the Working Group on Sepsis-Related Problems of the European Society of Intensive Care Medicine. Intensive Care Med. 1996;22(7):707-710.

42. Lim WS, van der Eerden MM, Laing R, Boersma WG, Karalus N, Town GI, Lewis SA, Macfarlane JT. Defining community acquired pneumonia severity on presentation to hospital: an international derivation and validation study. Thorax 2003;58(5):377-382.

43. Katz S, Ford AB, Moskowitz RW, Jackson BA, Jaffe MW. Studies of illness in the aged. The Index of ADL: a standardized measure of biological and psychosocial function. JAMA 1963;185:914-919.

44. Charlson ME, Pompei P, Ales KL, MacKenzie CR. A new method of classifying prognostic comorbidity in longitudinal studies: development and validation. J Chronic Dis. 1987;40(5):373-383.

45. Teasdale G, Jennett B. Assessment of coma and impaired consciousness. A practical scale. Lancet (London, England). 1974;2(7872):81-84.

46. Stiell IG, Wells GA. Methodologic standards for the development of clinical decision rules in emergency medicine. Ann Emerg Med. 1999;33(4):437-447.

47. Steyerberg EW. Clinical prediction models : a practical approach to development, validation, and updating. In. New York: Springer; 2009.

48. Magdelijns FJ, Schepers L, Pijpers E, Stehouwer CD, Stassen PM. Unplanned readmissions in younger and older adult patients: the role of healthcare-related adverse events. Eur J Med Res. 2016;21(1):35.

49. de Gelder J, Lucke JA, de Groot B, Fogteloo AJ, Anten S, Mesri K, Steyerberg EW, Heringhaus C, Blauw GJ, Mooijaart SP. Predicting adverse health outcomes in older emergency department patients: the APOP study. Neth J Med. 2016;74(8):342-352. 


\section{Supplemental data}

\section{Questionnaire 1: Emergency department questionnaire for the patient or caregiver}

Date:

Patient identification:

Time of completion:

- Answered by: patient / caregiver

- If caregiver: spouse/ child / acquaintance

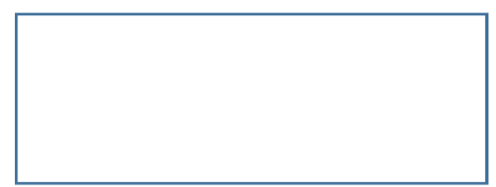

Please circle your answers

The following questions are related to the health of the patient:

1.How severely ill are you / do you find this patient?

$\begin{array}{lllll}1 & 2 & 3 & 4 & 5\end{array}$

$1=$ not at all, 2 = mildly, 3 =moderately, 4 = very, 5 = extremely

2a. Are you concerned about your (his/her) condition? $\begin{array}{llllll}1 & 2 & 3 & 4 & 5\end{array}$

$1=$ not at all, $2=$ a bit, 3 =moderately, 4 = very, 5 = extremely

2b. If so (score 2-5), what are you concerned about? 
3. Are you concerned about loss of independency 1 2 2 3 4 5 after this hospital visit (e.g. that you are not able to live at your own home anymore)?

$1=$ not at all, 2 = a bit, 3 =moderately, 4 =very, 5 = extremely

Please fill out this next question if the patient is answering the questions:

4. How would you describe your health before your visit to the emergency department?

$1=$ very good, 2 = good, 3 =fair, $4=$ poor, 5 = very poor 


\section{Questionnaire 2: Emergency department questionnaire for the nurse}

Date :

Patient identification:

Time of completion:

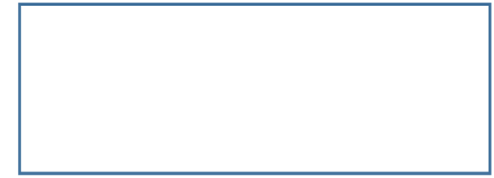

Please circle your answers

- Emergency nurse in training / emergency nurse

- Years of experience:

Please fill in this questionnaire after knowledge of the vital parameters, reason for ED visit and based on your first clinical impression before history taking, physical examination and additional tests.

1. How severely ill do you find this patient? 1 2 3 4 5

$1=$ not at all, $2=$ mildly, $3=$ moderately, $4=$ very, $5=$ extremely

2a. Are you concerned about this patient?

$\begin{array}{lllll}1 & 2 & 3 & 4 & 5\end{array}$

$1=$ not at all, 2 = a bit, 3 =moderately, $4=$ very, $5=$ extremely

2b. If so (score 2-5), what are you concerned about?

口 complications

$\square$ death

ㅁ loss of independency

ㅁother.. 
3. Would you be surprised if this patient

1 died within the next 12 months?

1 = yes, 2 = probably yes, $3=1$ don't know, 4 = probably no, $5=$ no

4. Would you be surprised if this patient died within the next 30 days?

1

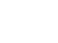

2

3

4

5

1 =yes, 2 = probably yes, $3=1$ don't know, $4=$ probably no, $5=$ no

5. Do you think this patient will be admitted to the hospital?

$\begin{array}{lllll}1 & 2 & 3 & 4 & 5\end{array}$

1 = no, 2 = probably not, $3=1$ don't know, 4 = probably yes, 5 = yes

6. When you think this patient will be admitted;

a. Do you think this patient needs intensive or medium care?

b. Do you think this patient will be admitted for longer than 7 days?

c. Do you think this patient will lose independency (e.g. is not able to live at his own home anymore)?
1

2

3

4

5

1

2

3

5

1

2

3

4

5 


\section{Questionnaire 3: Emergency department questionnaire for the}

Date:

Patient identification:

Time of completion:

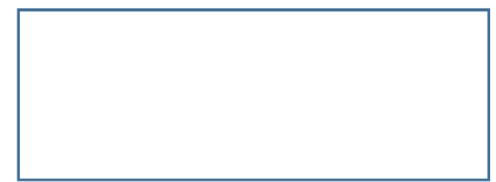

Please circle the answers.

- Emergency physician in training / emergency physician / resident internal medicine / Internist / Gastroenterologist

- Years of experience

Please fill in this questionnaire after knowledge of the vital parameters, reason for ED visit and based on your first clinical impression before history taking, physical examination and additional tests.

1. How severely ill do you find this patient?

1

2

3

4

5

$1=$ not at all, $2=$ mildly, $3=$ moderately, $4=$ very, $5=$ extremely

2a. Are you concerned about this patient?

$\begin{array}{lllll}1 & 2 & 3 & 4 & 5\end{array}$

$1=$ not at all, $2=\mathrm{abit}, 3=$ moderately, $4=$ very, $5=$ extremely

2b. If so (score 2-5), what are you concerned about?

口 complications

$\square$ death

口 loss of independency

$\square$ other. 
3. Would you be surprised if this patient 1 2

$\begin{array}{llll}2 & 3 & 4 & 5\end{array}$

$1=$ no, $2=$ probably not, $3=1$ don't know 4 = probably yes, 5 = yes

6. When you think this patient will be admitted;

a. Do you think this patient needs intensive or medium care?

1

1

$\begin{array}{lll}2 & 3 & 4\end{array}$

b. Do you think this patient will be admitted for longer than 7 days?

$\begin{array}{lllll}1 & 2 & 3 & 4 & 5\end{array}$

c. Do you think this patient will lose independency (e.g. is not able to live at his own home anymore)?

$\begin{array}{lllll}1 & 2 & 3 & 4 & 5\end{array}$

$1=$ no, $2=$ probably not, $3=1$ don't know, 4 = probably yes, 5 = yes 


\section{Questionnaire 4: Functional capability assessment questionnaire}

Date:

Patient identification:

Completed by: Research Investigator/Physician/Nurse

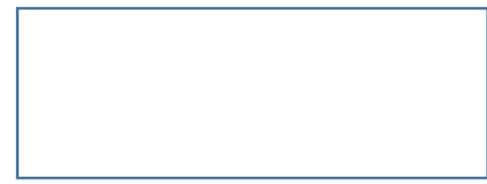

- The questions are about the functional capability of the patient 2 weeks before admission

\begin{tabular}{|l|l|l|}
\hline & Question & Answer \\
\hline $1^{*}$ & $\begin{array}{l}\text { Do you receive help during bathing? } \\
\text { (you may use help for a single part of the body) }\end{array}$ & Yes / No \\
\hline $2^{*}$ & $\begin{array}{l}\text { Can you dress yourself without help? } \\
\text { (you may have help tying shoes) }\end{array}$ & Yes / No \\
\hline $3^{*}$ & $\begin{array}{l}\text { Can you go to the bathroom, use it and return without any help? } \\
\text { (you may use a cane or walker) }\end{array}$ & Yes / No \\
\hline $4^{*}$ & $\begin{array}{l}\text { Can you move in and out of bed or chair without any help? } \\
\text { (you may use a cane or walker) }\end{array}$ & Yes / No \\
\hline $5^{*}$ & Are you completely continent for urine and feces? & Yes / No \\
\hline $6^{*}$ & $\begin{array}{l}\text { Can you eat without help? } \\
\text { (you may have help with food preparation) }\end{array}$ & Yes / No \\
\hline 7 & $\begin{array}{l}\text { Before hospital admission, did you need assistance for IADL (e.g., assistance in } \\
\text { housekeeping, preparing meals, shopping, etc.) on a regular basis? }\end{array}$ & Yes / No \\
\hline 8 & Do you use a walking device (e.g. a cane, rollator, walking frame, crutches, etc.)? & Yes / No \\
\hline 9 & Do you need assistance for travelling? & Yes / No \\
\hline 10 & Did you continue education after age 14? & Yes / No \\
\hline
\end{tabular}

* Definition of independency or dependency for these activities was conform the Katz Index of Independence in Activities of Daily Living

${ }^{1}$ Katz S et. al., JAMA, 1963 


\section{Chapter 3}

Short-term mortality in older medical emergency patients can be predicted using clinical intuition: A prospective study

N. Zelis, A.N. Mauritz, L.I.J.Kuijpers, J. Buijs, P.W. de Leeuw, P.M. Stassen 


\section{Abstract}

\section{Background}

Older emergency department (ED) patients are at risk for adverse outcomes, however, it is hard to predict these. We aimed to assess the discriminatory value of clinical intuition, operationalized as disease perception, self-rated health and first clinical impression, including the 30-day surprise question (SQ: "Would I be surprised if this patient died in the next 30 days" of patients, nurses and physicians. Endpoints used to evaluate the discriminatory value of clinical intuition were short-term (30-day) mortality and other adverse outcomes (intensive/medium care admission, prolonged length of hospital stay, loss of independent living or 30-day readmission).

\section{Methods}

In this prospective, multicentre cohort study, older medical patients ( $\geq 65$ years), nurses and physicians filled in scores regarding severity of illness and their concerns (i.e. disease perception and clinical impression scores) immediately after arrival of the patient in the ED. In addition, patients filled in a self-rated health score and nurses and physicians answered the SQ. Area under the curves (AUCS) of receiver operating characteristics (ROCs) were calculated.

\section{Results}

The median age of the 602 included patients was 79 years and $86.7 \%$ were community dwelling. Within 30 days, 66 (11.0\%) patients died and 263 (43.7\%) patients met the composite endpoint. The severity of concern score of both nurses and physicians yielded the highest AUCs for 30 -day mortality (for both $0.75 ; 95 \% \mathrm{Cl} 0.68-0.81$ ). AUCs for the severity of illness score and SQ of nurses and physicians ranged from 0.71 to 0.74 while those for the disease perception and self-rated health of patients ranged from 0.64 to 0.69 . The discriminatory value of the scores for the composite endpoint was lower (AUCs ranging from 0.60 to 0.67 ). We used scores that have not been previously validated which could influence their generalisability.

\section{Conclusion}

Clinical intuition, - disease perception, self-rated health and first clinical impression documented at an early stage after arrival in the ED, is a useful clinical tool to predict mortality and other adverse outcomes in older ED patients. Highest discriminatory values were found for the nurses' and physicians' severity of concern score. Intuition may be helpful for the implementation of personalised medical care in the future. 


\section{Introduction}

After an emergency department (ED) visit, older patients experience high rates of hospitalisation, functional decline, readmission and mortality. ${ }^{1,2}$ Important decisions concerning diagnostics and treatment have to be made within a short timeframe during an ED visit. Therefore, accurate identification of high risk patients is essential for optimal clinical care and safety. At this moment, reliable ways to predict adverse outcomes in older patients with generic problems are lacking. ${ }^{3,4}$

Clinical intuition can be a way to predict adverse outcomes in older ED patients. Indeed, the clinical impression of both nurses and physicians was associated with adverse outcomes. ${ }^{5-9}$ The disease perception by patients ${ }^{10}$ and self-rated health ${ }^{11-13}$ were associated with both morbidity and mortality as well. In addition, the surprise question (SQ) "Would I be surprised if this patient died in the next 12 months?" was found to predict adverse outcomes, although its reported accuracy varied widely. ${ }^{14,15}$ Most studies regarding clinical intuition were performed in younger patients ${ }^{6,8-10}$, clinical settings other than the ED (e.g. admission units ${ }^{6,16}$ and Intensive Care Units $\left(\right.$ ICUs) ${ }^{8}$ ), in selected groups of patients (e.g. patients with sepsis, cancer, renal failure or non-specific complaints $\left.{ }^{7,9,14}\right)$, and they used long-term mortality as endpoint. ${ }^{11,12,14}$ Moreover, most studies were performed with professionals who had access to results of physical examination and diagnostic test results and therefore, the first clinical impression was not tested.

Therefore, the value of clinical intuition; disease perception, self-rated health and first clinical impression, in predicting short-term mortality and other adverse outcomes remains unknown. We hypothesize that clinical intuition predicts adverse outcomes in older ED patients. To test this hypothesis, we set up a prospective multicentre cohort study to assess the discriminatory value of clinical intuition with respect to 1 ) shortterm (30-day) mortality and 2) other adverse outcomes such as ICU or medium care unit (MCU) admission, prolonged hospital stay (LOS), loss of independent living and unplanned readmission. 


\section{Materials and methods}

\section{Study design and setting}

This study is part of the Risk Stratification in the Emergency Department in Acutely ill Older Patients (RISE UP) study, a prospective multicentre observational cohort study that aims to identify predictors of adverse outcomes in older medical ED patients. This study was conducted at the EDs in Zuyderland Medical Centre (MC) and Maastricht University Medical Centre+ (MUMC+) in The Netherlands. These hospitals are both teaching hospitals, the first providing secondary, the second providing both secondary and tertiary care. This study was approved by the medical ethics committees of Zuyderland MC and MUMC+ (NL55867.096.15) and is registered on clinicaltrials.gov (NCT02946398).

\section{Study population}

All older ( $\geq 65$ years) medical ED patients who were assessed and treated under supervision of internists or gastroenterologists were eligible for inclusion. Internal medicine residents and emergency physicians included the patients in Zuyderland MC from July 2016 until February 2017 and in MUMC+, from September 2016 until February 2017. All patients or their legal representatives signed an informed consent form before study entry. We excluded patients who already participated in the study, who were unable to speak Dutch, German or English or who were admitted to a ward of another specialty than internal medicine or gastroenterology. We assumed that in certain circumstances the physicians would give priority to emergency care and that not all possible candidates could be asked to participate in this study. This turned out to be true, and therefore, the patients formed a convenience sample. Because of this finding, we evaluated possible selection bias by analysing data of non-included patients.

\section{Data collection and measurements}

Data were collected from the patients' electronic medical records and questionnaires. Immediately after arrival, all participants received a questionnaire, which was filled in by the patient or caregiver. The attending nurse and physician completed a questionnaire at the same moment, immediately after obtaining informed consent and before history taking, physical examination and without knowledge of any diagnostic results. All respondents filled in the questionnaires independent of each other to ensure blinding of the results. Questions about disease perception, self-rated 
health and first clinical impression including the SQ were scored using a Likert scale from 1 to 5 (S3.1 File). In this manuscript, we will refer to these questions as scores, except for the SQ.

The questionnaires of patients, nurses and physicians consisted of three identical questions: 1) "How severely ill are you/do you find this patient?"(severity of illness score) and 2) "Are you concerned about your/her/his condition?" (severity of concern score) 3) "Are you concerned about loss of independency after this hospital visit?" for patients and "Do you think this patients will lose independency?" for nurses and physicians (loss of independency score). Patients or their family members/caregivers had to answer one additional question: "How would you describe your health before your visit to the ED?" (self-rated health score ${ }^{13}$ ). Nurses and physicians answered three additional questions. The first additional question was the 30-day $\mathrm{SQ}^{17}$ : "Would you be surprised if this patient died in the next 30 days?". The second question was: "Do you think this patient will be admitted for more than 7 days?" (length of hospital stay (LOS) score). Third, the number of years of experience was asked.

We collected data on demographics, living situation, comorbidities (according to the Charlson Comorbidity Index ${ }^{18}$ ) and cognitive function from medical records. Functional status was assessed using a questionnaire to calculate the Katz Activities of Daily Living $(A D L)$ index score ${ }^{19}$ in all hospitalised patients. We recorded the main reason for the ED-visit according to the International Classification of Diseases (ICD)10 system. $^{20}$

To analyse possible selection bias, for 200 patients who were not included in this study, we collected the abovementioned data, except for data on the questionnaires and the Katz-ADL index score. Demographics were collected for all possible candidates during the study period.

\section{Primary and secondary outcome measures}

The primary endpoint to evaluate the discriminatory value of clinical intuition was 30-day all-cause mortality, for the severity of illness, severity of concern, SQ and selfrated health score. The secondary endpoint was a composite endpoint consisting of admission to ICU/MCU, prolonged LOS ( $>7$ days), loss of independent living and unplanned readmission within 30 days after discharge. This composite endpoint was used to assess the value of the severity of illness, severity of concern and self-rated health score. Prolonged LOS and loss of independent living were calculated as single secondary endpoints as well. For the analysis of prolonged LOS, patients who were not admitted to the hospital or who died within seven days during hospital stay were 
excluded. Loss of independent living was defined as discharge to a nursing home/hospice/revalidation clinic or palliative care in previously community dwelling patients. For the analysis of loss of independent living, only patients who were discharged alive and who were not fully dependent (i.e. living in nursing home) before admission were included. Patients who died during the first admission or within 30 days after discharge before being readmitted were excluded in the analysis of readmissions.

\section{Statistical analysis}

We performed a descriptive analysis of baseline characteristics of included patients and endpoints. Continuous variables were reported as means with standard deviations or medians with interquartile ranges (IQRs) and categorical variables as proportions. In case of missing values, valid percentages were used.

The discriminatory value of the disease perception, self-rated health and clinical impression scores was analysed by calculating the area under the curves (AUCs) of receiver operating characteristics (ROCs) with 95\% confidence intervals (Cls). An AUC of 0.9-1.0 was considered as being excellent, 0.8-0.9 very good, 0.7-0.8 good, 0.6-0.7 sufficient and 0.5-0.6 as bad accuracy. ${ }^{21}$ The method of DeLong ${ }^{22}$ was used to test for significant differences between AUCs of correlated patients', nurses' and physicians' scores.

Sensitivity, specificity, positive predictive values (PPVs) and negative predictive values (NPVs) were calculated for different cut-off values of the scores. An optimum cut-off value was chosen based on the value being closest to the upper left corner of the AUC. When two values were equally distanced, the value with the highest Youden's Index $^{21}$ was selected. Both positive and negative likelihood ratios (LRs) were calculated. For the SQ, loss of independency score and LOS score of nurses and physicians, score 1 and 2 were considered negative and score 4 and 5 positive. Score 3, "I don't know", was not included in the analysis.

The interrater reliability of the scores was calculated using the intraclass correlation coefficient (ICC) using a two-way random model for patients, nurses and physicians. ${ }^{23}$ An ICC under 0.40 was considered poor, between 0.40-0.59 fair, 0.60-0.74 good and between $0.75-1.00$ excellent. $^{24}$ LRs for 30-day mortality were calculated for cases in which patients, nurses and physicians agreed or disagreed.

All analyses were performed using IBM SPSS Statistics for Windows, Version 24.0 (IBM Corp., Armonk, N.Y., USA) and R version 3.4.4. p-values <0.05 were considered statistically significant. 


\section{Results}

\section{Study population}

During the study period, 2109 older patients (1175 patients in Zuyderland MC and 934 patients in MUMC+) visited the ED and were treated by internists or gastroenterologists (Figure 3.1). In total, 1506 (71.4\%) patients were not included because 1$)$ they were not asked to participate $(n=1274,60.4 \%) 2$ ) they refused to give informed consent $(n=168,8.0 \%), 3)$ they were unable to give informed consent ( $n=56$, $2.7 \%)$ or 4 ) there was a language barrier ( $n=8,0.4 \%)$. In total, $603(28.6 \%)$ patients were enrolled in this study. Since for one patient all questionnaires were missing, 602 patients were included in the final analysis.

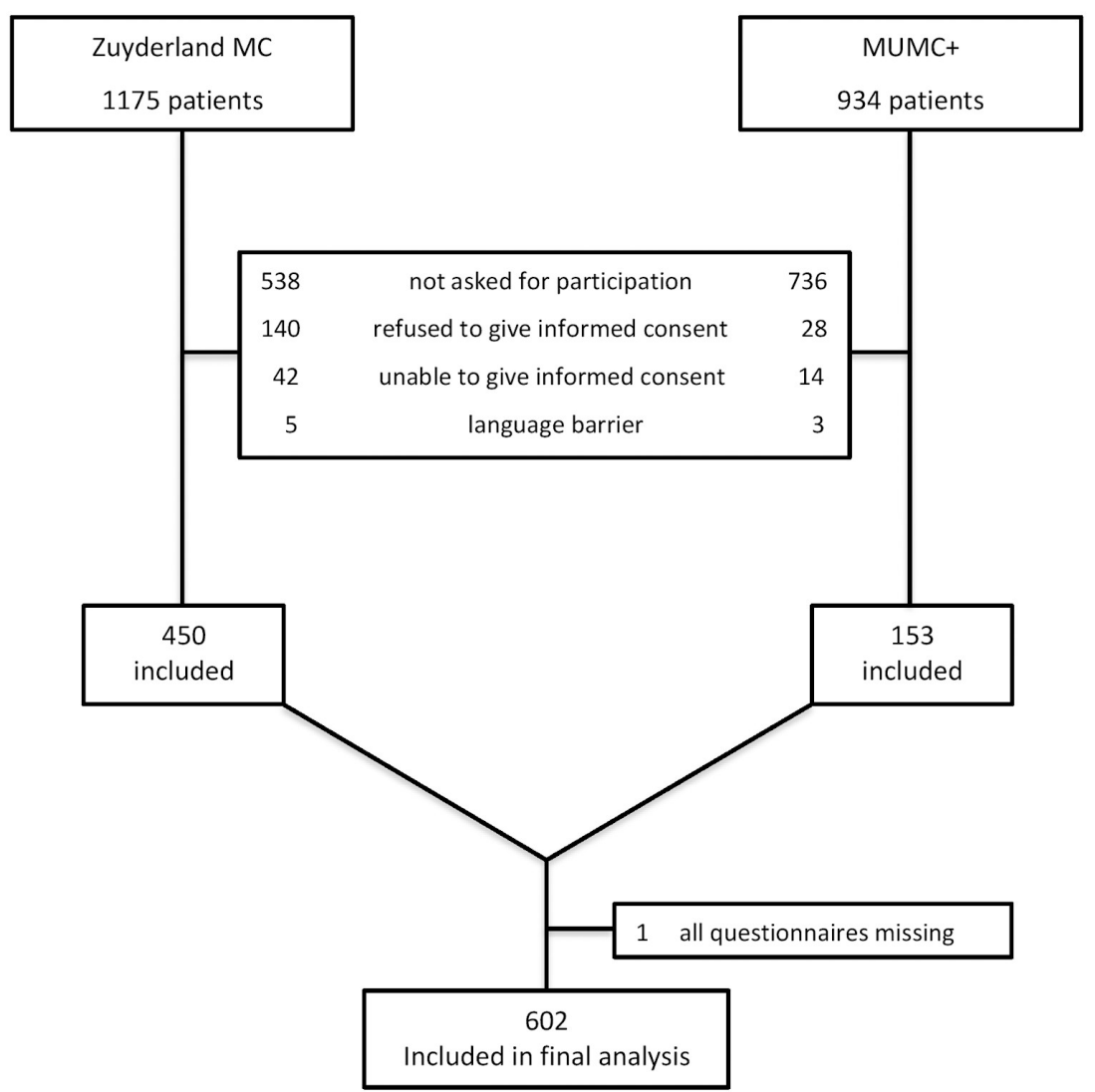

Figure 3.1 Flowchart of Zuyderland MC and MUMC+ patients. 


\section{Patient characteristics and questionnaires}

The median age of the study population was 79 years (73-85) and $51.7 \%$ were male (Table 3.1). Most patients were community-dwelling (86.7\%) and the median Katz-ADL score was 0 [0-2]. Nurses and physicians had a median professional experience of 3 (IQR: 1-15) and 2 (IQR 1-3) years, respectively.

Patients or their caregivers filled in 594 (99.0\%) questionnaires (91.1\% completely), nurses 568 (94.7\%; 99.3\% completely) and physicians 597 questionnaires (99.5\%; $99.8 \%$ completely).

Table 3.1 Characteristics of patients and professionals ${ }^{a}$.

\begin{tabular}{lc}
\hline & All patients \\
& $\mathbf{N}=602$ \\
\hline Demographics & $79(73-85)$ \\
Age (years), median (IQR) & $311(51.7)$ \\
Male & \\
Living situation & $522(86.7)$ \\
Community-dwelling & $50(8.3)$ \\
Nursing- and care home & $30(5.0)$ \\
Other & \\
Comorbidity and functional status & $2(1-3)$ \\
Charlson comorbidity index score, median (IQR) & $0(0-2)$ \\
Katz-ADL index scoreb ${ }^{\text {b }}$ median (IQR) & \\
Reason for ED visit (ICD-10) & $176(29.2)$ \\
Infectious diseases & $156(25.9)$ \\
Diseases of the digestive system & $55(9.1)$ \\
Diseases of the circulatory system & $52(8.6)$ \\
Neoplasms & $31(5.1)$ \\
Endocrine, nutritional and metabolic diseases & $30(5.0)$ \\
Diseases of the respiratory system & $28(4.7)$ \\
Diseases of the blood and blood-forming organs & $28(4.7)$ \\
Diseases of the genitourinary system & $46(7.6)$ \\
Miscellaneous & $478(79.4)$ \\
Admission &
\end{tabular}

ADL, Activities of Daily Living; ICD-10, International Classification of Diseases-10; IQR, interquartile range ${ }^{a}$ Values are numbers (percentages) unless stated otherwise. Incomplete data for: Katz-ADL index score

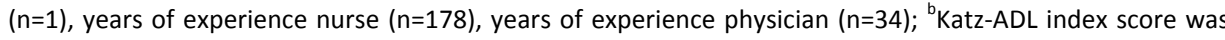
calculated for all hospitalized patients.

\section{Predictive ability of the scores for 30-day mortality and the composite endpoint}

In total, 66 (11.0\%) patients died within 30 days of their ED visit and 263 patients (43.7\%) met the composite endpoint (Table 3.2). For all scores, 30-day mortality 
increased with increasing value (Figure 3.2). For the composite endpoint, a similar pattern was seen (Figure 3.3a).

Table 3.2 Outcomes of study population ${ }^{\mathrm{a}}$.

\begin{tabular}{lc}
\hline Outcomes & All patients \\
& $\mathbf{N}=\mathbf{6 0 2}$ \\
\hline 30-day all-cause mortality & $66(11.0)$ \\
ICU or MCU admission & $26(4.3)$ \\
Prolonged LOS & $184(40.4)^{\mathrm{b}}$ \\
Loss of independent living & $76(18.0)^{\mathrm{b}}$ \\
Readmission within 30 days of discharge & $83(15.7)^{\mathrm{b}}$ \\
Composite endpoint & $263(43.7)$ \\
\hline
\end{tabular}

ICU, intensive care unit; LOS, length of hospital stay; MCU, medium care unit. ${ }^{a}$ Values are numbers (percentages) unless stated otherwise; ${ }^{b}$ Denominator for prolonged LOS: 456; for loss of independent living: 422 and for readmission: 527.

For 30-day mortality, the AUCs were lower for the patients' disease perception and self-rated health scores (AUCs ranging from 0.64 to 0.69 ) than for the clinical impression scores of nurses and physicians (AUCs ranging from 0.71 to 0.75 , Table 3.3). The nurses' and physicians' severity of concern scores yielded the highest AUCs (0.75; 95\% Cl 0.68-0.81 for both) and both were significantly higher than the patients' severity of concern score. For all scores, AUCs for mortality were higher than those for the composite endpoint (AUCs ranging from 0.60 to 0.67 ).

For patients, the scores yielded higher sensitivity (around 75\%) than specificity (around 50\%) with respect to 30-day mortality (Table 3.3). For nurses and physicians, sensitivity was lower (about $50 \%$ ) than specificity (around $85 \%$ ). The only exception was the nurses' severity of concern score, revealing high sensitivity (85.9\%) and low specificity (54.7\%). NPVs were high (around 94\%) for all scores whereas PPVs were higher for nurses and physicians than for patients (around $25-30 \%$ and $15 \%$ resp.). Positive likelihood ratios (LRs+) for 30-day mortality were higher for the scores of nurses and physicians than for patients, while negative likelihood ratios (LRs-) were comparable between the three groups.

For the composite endpoint, the patients' scores yielded a lower sensitivity but higher specificity compared to the prediction of mortality. For both nurses and physicians, sensitivity was higher for the composite endpoint than for mortality, while specificity was lower (around 60\%). For the composite endpoint, LRs+ were lower for nurses' and physicians' scores than for mortality. 


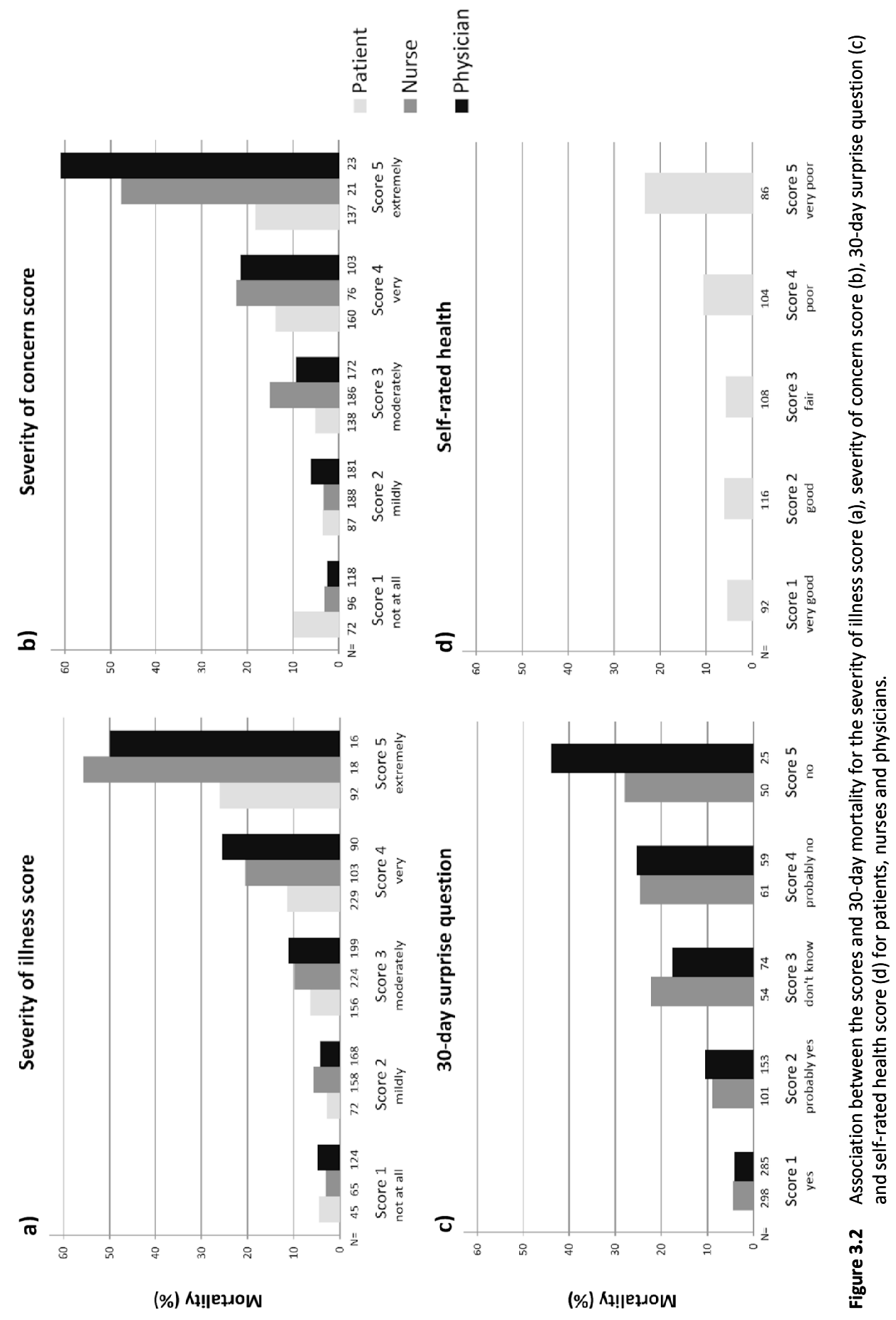




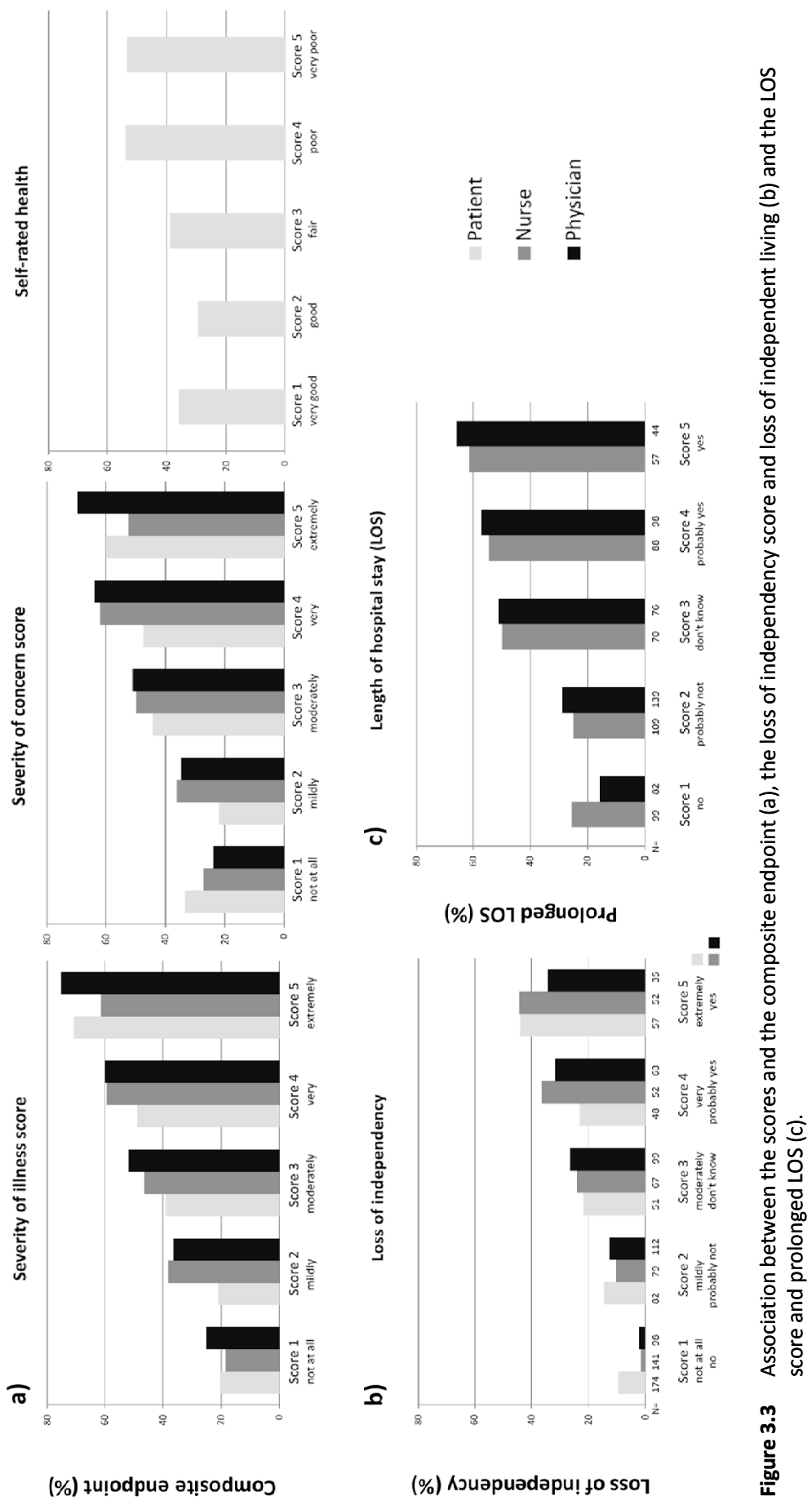


Table 3.3 Predictive ability of the scores.

\begin{tabular}{|c|c|c|c|c|c|c|c|c|}
\hline \multirow{2}{*}{$\begin{array}{l}\text { Corresponding score and } \\
\text { outcome }\end{array}$} & \multicolumn{2}{|l|}{ Without cut-off value } & \multicolumn{4}{|c|}{ With cut-off value } & \multirow[b]{2}{*}{$\mathrm{LR}+$} & \multirow[b]{2}{*}{ LR- } \\
\hline & $\mathrm{AUC}(95 \% \mathrm{Cl})$ & Cut-off & $\begin{array}{c}\text { Sensitivity } \\
(\%)\end{array}$ & $\begin{array}{c}\text { Specificity } \\
(\%)\end{array}$ & $\begin{array}{l}\text { PPV } \\
(\%)\end{array}$ & $\begin{array}{l}\text { NPV } \\
(\%)\end{array}$ & & \\
\hline \multicolumn{9}{|l|}{ Mortality } \\
\hline \multicolumn{9}{|l|}{ Severity of illness score } \\
\hline Patient & $0.69(0.62-0.76)$ & 4 & 78.1 & 48.9 & 15.6 & 94.9 & 1.5 & 0.5 \\
\hline Nurse & $0.71(0.64-0.78)$ & 4 & 48.4 & 82.1 & 25.6 & 92.6 & 2.7 & 0.6 \\
\hline Physician & $0.72(0.65-0.79)$ & 4 & 47.0 & 85.9 & 29.2 & 92.9 & 3.3 & 0.6 \\
\hline \multicolumn{9}{|l|}{ Severity of concern score } \\
\hline Patient & $0.64(0.56-0.71)$ & 4 & 73.4 & 52.8 & 15.8 & 94.3 & 1.6 & 0.5 \\
\hline Nurse & $0.75(0.68-0.81)^{\mathrm{a}}$ & 3 & 85.9 & 54.7 & 19.4 & 96.8 & 1.9 & 0.3 \\
\hline Physician & $0.75(0.68-0.81)^{\mathrm{a}}$ & 4 & 54.5 & 83.1 & 28.6 & 93.6 & 3.2 & 0.6 \\
\hline \multicolumn{9}{|l|}{ 30-day SQ } \\
\hline Nurse & $0.73(0.66-0.80)$ & 4 & 56.9 & 82.1 & 26.1 & 94.5 & 3.2 & 0.5 \\
\hline Physician & $0.74(0.68-0.81)$ & 4 & 49.1 & 87.6 & 31.0 & 93.8 & 4.0 & 0.6 \\
\hline \multicolumn{9}{|l|}{ Self-rated health score } \\
\hline Patient & $0.67(0.58-0.75)$ & 4 & 63.3 & 65.2 & 16.3 & 94.3 & 1.8 & 0.6 \\
\hline \multicolumn{9}{|l|}{ Composite Endpoint } \\
\hline \multicolumn{9}{|l|}{ Severity of illness score } \\
\hline Patient & $0.67(0.62-0.71)$ & 4 & 67.6 & 56.6 & 55.1 & 68.9 & 1.6 & 0.6 \\
\hline Nurse & $0.63(0.58-0.72)$ & 3 & 71.0 & 47.2 & 51.0 & 67.7 & 1.3 & 0.6 \\
\hline Physician & $0.65(0.61-0.69)$ & 3 & 64.8 & 59.5 & 55.4 & 68.5 & 1.6 & 0.6 \\
\hline \multicolumn{9}{|l|}{ Severity of concern score } \\
\hline Patient & $0.63(0.58-0.67)$ & 4 & 60.3 & 58.1 & 53.2 & 65.0 & 1.4 & 0.7 \\
\hline Nurse & $0.63(0.58-0.67)$ & 3 & 62.0 & 59.6 & 54.4 & 66.9 & 1.5 & 0.6 \\
\hline Physician & $0.67(0.62-0.71)$ & 3 & 65.1 & 61.9 & 57.0 & 69.6 & 1.7 & 0.6 \\
\hline \multicolumn{9}{|l|}{ Self-rated health score } \\
\hline Patient & $0.60(0.55-0.65)$ & 4 & 48.3 & 70.2 & 53.7 & 65.5 & 1.6 & 0.7 \\
\hline \multicolumn{9}{|l|}{ Loss of independent living } \\
\hline \multicolumn{9}{|l|}{ Loss of independency score } \\
\hline Patient & $0.69(0.62-0.76)$ & 3 & 62.7 & 76.7 & 30.1 & 89.1 & 2.7 & 0.5 \\
\hline Nurse & $0.81(0.75-0.86)^{\mathrm{a}}$ & 4 & 80.8 & 77.2 & 40.4 & 95.5 & 3.5 & 0.3 \\
\hline Physician & $0.72(0.66-0.78)$ & 4 & 66.7 & 74.6 & 28.8 & 92.4 & 2.6 & 0.5 \\
\hline \multicolumn{9}{|l|}{ Prolonged LOS } \\
\hline \multicolumn{9}{|l|}{ LOS score } \\
\hline Nurse & $0.67(0.61-0.72)$ & 4 & 61.5 & 71.6 & 57.2 & 75.0 & 2.2 & 0.5 \\
\hline Physician & $0.70(0.65-0.75)$ & 4 & 61.6 & 74.7 & 59.9 & 76.0 & 2.4 & 0.5 \\
\hline
\end{tabular}

AUC, area under the curve; $\mathrm{Cl}$, confidence interval; PPV, Positive Predictive Value; NPV, Negative Predictive Value; LR+, positive likelihood ratio; LR-, negative likelihood ratio; SQ, surprise question; LOS, length of hospital stay. ${ }^{\text {a } A U C}$ is significantly higher than the other AUCs for the same score.

\section{Predictive ability of the scores for loss of independent living and prolonged LOS}

Loss of independent living was documented in 76 (18.0\%) patients and 184 (40.4\%) patients were admitted longer than 7 days (prolonged LOS, Table 3.2). With increasing scores, more patients met these endpoints (Figure 3.3b and 3.3c). The AUC of the 
nurses' loss of independency score was very good (AUC: 0.81), and significantly higher than the patients' and physicians' score (AUC: 0.69 and 0.72 resp., P-value $<0.05$ ). For the other scores, AUCs were lower (around 0.70).

Both sensitivity (80.8\%) and specificity $(77.2 \%)$ were high for the nurses' loss of independency score and this resulted in a LR+ of 3.5 and LR- of 0.3 . For the other scores, both sensitivity and specificity were lower.

\section{Interrater reliability}

The interrater reliability between patients and the healthcare professionals was poor with intraclass correlation coefficients (ICCS) ranging from 0.12 to 0.37 for the three scores (Table 3.4). We obtained a fair interrater reliability between nurses and physicians (ICCs around 0.50) for all of the four scores, except for the SQ that yielded a poor ICC of 0.33 .

Table 3.4 Intraclass correlation coefficients with $95 \% \mathrm{Cl}$ for the different scores.

\begin{tabular}{lc}
\hline Comparison & ICC (95\% CI) \\
\hline Patient-Nurse & $0.31(0.13-0.45)$ \\
Severity of illness score & $0.20(0.07-0.32)$ \\
Severity of concern score & $0.28(0.20-0.36)$ \\
Loss of independency score & \\
Patient-Physician & $0.30(0.05-0.49)$ \\
Severity of illness score & $0.23(0.09-0.34)$ \\
Severity of concern score & $0.37(0.30-0.44)$ \\
Loss of independency score & \\
Nurse-Physician & $0.50(0.43-0.56)$ \\
Severity of illness score & $0.46(0.40-0.53)$ \\
Severity of concern score & $0.33(0.26-0.40)$ \\
30-day SQ & $0.50(0.43-0.56)$ \\
Loss of independency score & \\
\hline
\end{tabular}

ICC, intraclass correlation coefficient; $\mathrm{Cl}$, confidence interval; $\mathrm{SQ}$, surprise question.

\section{Predictive ability of combined scores}

Combination of scores of patients, nurses and physicians yielded higher LRs+ and LRswhen there was agreement (Table 3.5). When extreme scores (i.e. score 1 or score 5) of patients, nurses and physicians were combined, LRs highly improved. The extreme scores, however, were only encountered in a limited number of patients, except when score 1, "Yes, I would be surprised", was given for the SQ ( $n=188)$.

Disagreement between nurses and physicians was less common (around 20\%) than agreement. When nurses and physicians disagreed, LRs were low and around 1.0-1.5, 
except for the severity of concern score, where the LR was 0.3 when a physician was concerned but the nurse disagreed.

Table 3.5 Likelihood ratios of combined scores for 30-day mortality.

\begin{tabular}{|c|c|c|c|c|c|c|}
\hline Combination of scores & & & & $\mathbf{n}$ & LR & $\begin{array}{c}\text { Observed } \\
\text { mortality (\%) }\end{array}$ \\
\hline \multicolumn{7}{|l|}{ Severity of illness score } \\
\hline \multicolumn{7}{|l|}{ Agreement } \\
\hline & Patient + & Nurse + & Physician + & 47 & 6.0 & 42.6 \\
\hline & Patient - & Nurse - & Physician - & 214 & 0.3 & 3.7 \\
\hline & & Nurse + & Physician + & 58 & 5.1 & 39.7 \\
\hline & & Nurse - & Physician - & 399 & 0.5 & 6.3 \\
\hline & & Nurse score 5 & Physician score 5 & 7 & 47.0 & 85.7 \\
\hline & & Nurse score 1 & Physician score 1 & 33 & 0.3 & 3.0 \\
\hline \multicolumn{7}{|l|}{ Disagreement } \\
\hline & & Nurse + & Physician - & 63 & 1.1 & 12.7 \\
\hline & & Nurse - & Physician + & 45 & 1.7 & 17.8 \\
\hline \multicolumn{7}{|l|}{ Severity of concern score } \\
\hline \multicolumn{7}{|c|}{ Agreement } \\
\hline & Patient + & Nurse + & Physician + & 68 & 5.9 & 42.6 \\
\hline & Patient - & Nurse - & Physician - & 156 & 0.2 & 1.9 \\
\hline & & Nurse + & Physician + & 94 & 4.4 & 36.2 \\
\hline & & Nurse - & Physician - & 256 & 0.3 & 3.1 \\
\hline & & Nurse score 5 & Physician score 5 & 8 & 23.5 & 75.0 \\
\hline & & Nurse score 1 & Physician score 1 & 43 & 0.4 & 4.7 \\
\hline \multicolumn{7}{|l|}{ Disagreement } \\
\hline & & Nurse + & Physician - & 188 & 1.0 & 11.2 \\
\hline & & Nurse - & Physician + & 26 & 0.3 & 3.8 \\
\hline \multicolumn{7}{|l|}{ Surprise question } \\
\hline \multicolumn{7}{|l|}{ Agreement } \\
\hline & & Nurse + & Physician + & 34 & 10.7 & 52.9 \\
\hline & & Nurse - & Physician - & 319 & 0.4 & \\
\hline & & Nurse score 5 & Physician score 5 & 3 & $\infty$ & 100 \\
\hline & & Nurse score 1 & Physician score 1 & 188 & 0.1 & 1.6 \\
\hline \multicolumn{7}{|l|}{ Disagreement } \\
\hline & & Nurse + & Physician - & 57 & 1.6 & 14.0 \\
\hline & & Nurse - & Physician + & 36 & 0.9 & 8.3 \\
\hline
\end{tabular}

$\mathrm{n}$, number of patients with the corresponding scores; LR, likelihood ratio.

\section{Selection bias}

Table 3.6 shows the patient characteristics and outcomes of the included and nonincluded patients during study recruitment. The two groups were comparable regarding age and sex but clinical signs of cognitive impairment and delirium were less frequently present in included patients (29.1 vs. 38.6\%, resp.). Outcomes of included and non-included patients did not differ, although 30-day mortality was slightly lower for the included patients. 
Table 3.6 Overview of characteristics and outcomes of included and non-included patients ${ }^{a}$.

\begin{tabular}{lccc}
\hline & $\begin{array}{c}\text { Included } \\
\text { Prospectively } \\
\text { analysed } \\
\mathbf{N = 6 0 2}\end{array}$ & $\begin{array}{c}\text { Non-included } \\
\text { Retrospectively } \\
\text { analysed } \\
\mathbf{N = 2 0 0}\end{array}$ & $\begin{array}{c}\text { Total } \\
\text { All possible } \\
\text { candidates } \\
\mathbf{N = 2 1 0 9}\end{array}$ \\
\hline Demographics & $79(73-85)$ & $78(72-85)$ & $78(71-84)$ \\
Age (years), median (IQR) & $311(51.7)$ & $100(50.0)$ & $1023(48.5)$ \\
Male & $522(86.7)$ & $155(78.7)$ & \\
Living community dwelling & $50(8.3)$ & $23(11.7)$ & \\
Living in nursing/care home & $2(1-3)$ & $2(1-3)$ & \\
Comorbidity and cognitive functioning & $167(29.1)$ & $66(38.6)$ & \\
Charlson comorbidity index score & & & \\
Cognitive impairment or delirium & $66(10.9)$ & $30(15.0)$ & \\
Outcomes & $263(43.7)$ & $84(42.0)$ & \\
30-day mortality & & \\
Composite endpoint & & & \\
\hline
\end{tabular}

${ }^{a}$ Values are numbers (percentages) unless stated otherwise. Incomplete data in the prospective and retrospective cohort for: living situation ( $n=0$ and $n=3$, resp.), cognitive impairment or delirium ( $n=28$ and $n=29$, resp.) and outcomes ( $n=0$ and $n=1$, resp.).

\section{Discussion}

To the best of our knowledge, this is the first prospective study examining the discriminatory value of clinical intuition - disease perception, self-rated health and the first clinical impression - with respect to short-term mortality and other adverse outcomes in older ED patients. We found that 30-day mortality can be predicted by application of the clinical intuition questionnaire for patients, nurses and physicians early after arrival in the ED. The best discriminatory values were encountered for the nurses' and physicians' severity of concern score (AUCs of 0.75 ). When patients and professionals were in agreement, diagnostic accuracy further improved. The discriminatory value of the first clinical impression, disease perception and self-rated health for the composite endpoint was lower, but still sufficient (AUCs ranging from 0.60 to 0.67 ), compared to the prediction of 30 -day mortality. Loss of independent living was best predicted by nurses.

\section{Comparison with previous studies}

Our findings concerning the discriminatory value of the first clinical impression of nurses and physicians are in line with other studies. A Swiss study ${ }^{7}$ showed that the first clinical impression of physicians was predictive for 30-day mortality with an AUC of 0.66 , which was substantially lower compared to our results (AUCs ranging from 0.71 to 0.75 ). This difference may be explained by the use of a different scoring 
system, inclusion of patients with non-specific complaints or selective exclusion of seriously ill patients in the Swiss cohort. In a Danish study ${ }^{6}$, nurses and physicians in a medical admission unit both predicted in-hospital mortality adequately (AUC 0.82 for nurses and 0.76 for physicians). In line with our study, they found a higher accuracy when both nurses and physicians agreed (76\%). This finding supports the importance of teamwork in emergency care. An explanation for the high AUCs found in this Danish study may be that in-hospital mortality was predicted when results of the first evaluation and diagnostics were available. We, however, decided to investigate the first clinical impression of nurses and physicians immediately after arrival in the ED at the moment that major decisions about prioritization of diagnostics and treatments have to be made. This judgement turned out to be accurate, even in a relatively unexperienced group of professionals.

The SQ turned out to be to be a good predictor of 30-day mortality. In a recent metaanalysis $^{15}$, the discriminatory value and specificity of the SQ for 30-day mortality in one study with septic ED patients was lower than in our study (AUC of 0.59 and specificity of $69 \%$ ). This may be explained by the fact that in-hospital and not 30-day mortality was used as outcome. Furthermore, it might be possible that differences between sepsis patients who are at risk of dying and those who are not are hard to make. Based on our data, we conclude that the 30-day SQ predicts 30-day mortality accurately in older medical ED patients.

The discriminatory value of the three scores regarding 30-day mortality was lower for patients than for professionals but this difference was, except for the severity of concern score, not significant. NPVs were high (around 94\%) for patients and professionals and LRs- were low when patients and professionals agreed. This indicates that when scores are negative, especially when in agreement, there is a low probability of mortality. This finding can support adequate decision making about dismission of patients from the ED. We recorded higher specificity, PPVs and LRs+ for nurses and physicians than for patients. This is not surprising, as professionals are confronted with acutely ill patients on a daily basis. The observation that patients frequently scored themselves very or extremely ill and that they were severely concerned is in line with this assumption. We found a poor interrater reliability between patients and professionals. These findings match with another Swiss study ${ }^{10}$ that found lower discriminatory values for the assessment by patients than by nurses or physicians. This Swiss study also found a poor interrater reliability between patients and professionals (ICC of 0.17 for patient/nurse and 0.07 for patient/physician). Nevertheless, when patients agreed with professionals, discriminatory values for mortality improved. In addition, sensitivity was higher for the patients' scores 
compared to that of professionals, which may support the importance of involvement of patients in clinical decision making.

In this study, we found lower discriminatory values for the composite endpoints than for mortality. This may be explained by the fact that the scores are based on the intuitive feeling on whether a patient is at risk of dying instead on being at risk of other outcomes. However, loss of independent living was accurately predicted by nurses (AUC 0.81) indicating that this specific score can be used as a screening instrument by nurses, in order to deliver appropriate care.

\section{Study limitations}

Our study has several limitations. First, we used scores, which have not been previously validated, including the severity of illness and severity of concern score. However, a comparable 9-point ${ }^{5}$ and 10-point score $^{25}$ was used in other studies, showing a similar association and discriminatory value for mortality. Nevertheless, future studies may be helpful to externally validate our scores and to test reproducibility. Second, both the nurses and physicians participating in this study had only a few years of professional experience, a factor that may be different in other EDs, hospitals or countries. It is possible that intuition is more predictive in more experienced professionals. Third, we tested the discriminatory value of the first clinical impression of nurses and physicians. We do not know whether this discriminatory ability improves after physical examination and diagnostics or in a later stage during hospital admission. On the other hand, we are convinced that a quick judgement in the ED is essential to optimize safe and personalized care. In the fourth place, our results may have been biased because of selective inclusion of patients since many patients were not asked to participate. For this reason we retrospectively compared the data of non-included candidates with our study population and did not find evidence for selection bias.

\section{Conclusions}

In conclusion, clinical intuition - disease perception, self-rated health and first clinical impression - predicts mortality and other adverse outcomes in older ED patients. Intuition is inexpensive and can identify severely ill patients in an early stage, which may contribute to timely and personalised treatment and aftercare. Diagnostic accuracy improves when patients and professionals are in agreement, which may support the importance of shared decision making. We think that professionals should be aware that their first impression is a valuable tool to predict clinical sequelae and 
can help them to apply personalized medical care. Studies are needed to validate our scores, to test their reproducibility and to test their ability to improve clinical outcome or well-being in older ED patients.

\section{Acknowledgments}

We thank I. Luu (MSc) for the design of the figures. In addition, we would like to thank all patients and medical staff of Zuyderland Medical Centre and Maastricht University Medical Centre+ who contributed to this study. 


\section{References}

1. Aminzadeh F, Dalziel WB. Older adults in the emergency department: a systematic review of patterns of use, adverse outcomes, and effectiveness of interventions. Ann Emerg Med. 2002;39(3):238-47.

2. Samaras N, Chevalley T, Samaras D, Gold G. Older patients in the emergency department: a review. Ann Emerg Med. 2010;56(3):261-9.

3. Grossmann FF, Zumbrunn T, Ciprian S, Stephan FP, Woy N, Bingisser R, et al. Undertriage in older emergency department patients--tilting against windmills? PloS One. 2014;9(8):e106203.

4. Carpenter CR, Shelton E, Fowler S, Suffoletto B, Platts-Mills TF, Rothman RE, et al. Risk factors and screening instruments to predict adverse outcomes for undifferentiated older emergency department patients: a systematic review and meta-analysis. Acad Emerg Med. 2015;22(1):1-21.

5. Charlson ME, Sax FL, MacKenzie CR, Fields SD, Braham RL, Douglas RG, Jr. Assessing illness severity: does clinical judgment work? J Chronic Dis. 1986;39(6):439-52.

6. Brabrand M, Hallas J, Knudsen T. Nurses and physicians in a medical admission unit can accurately predict mortality of acutely admitted patients: a prospective cohort study. PloS One. 2014;9(7): e101739.

7. Beglinger B, Rohacek M, Ackermann S, Hertwig R, Karakoumis-Ilsemann J, Boutellier S, et al. Physician's first clinical impression of emergency department patients with nonspecific complaints is associated with morbidity and mortality. Medicine. 2015;94(7):e374.

8. Sinuff T, Adhikari NK, Cook DJ, Schunemann HJ, Griffith LE, Rocker G, et al. Mortality predictions in the intensive care unit: comparing physicians with scoring systems. Crit Care Med. 2006;34(3):878-85.

9. van der Vegt $A E$, Holman $M$, ter Maaten JC. The value of the clinical impression in recognizing and treating sepsis patients in the emergency department. Eur J Emerg Med. 2012;19(6):373-8.

10. Rohacek $\mathrm{M}$, Nickel $\mathrm{CH}$, Dietrich $\mathrm{M}$, Bingisser R. Clinical intuition ratings are associated with morbidity and hospitalisation. Int J Clin Pract. 2015;69(6):710-7.

11. Bamia C, Orfanos P, Juerges H, Schottker B, Brenner H, Lorbeer R, et al. Self-rated health and all-cause and cause-specific mortality of older adults: Individual data meta-analysis of prospective cohort studies in the CHANCES Consortium. Maturitas. 2017;103:37-44.

12. Wong DD, Wong RP, Caplan GA. Self-rated health in the unwell elderly presenting to the emergency department. Emergency medicine Australasia : EMA. 2007;19(3):196-202.

13. Godard-Sebillotte C, Drame M, Basileu T, Fanon JL, Godaert L. Is self-rated health an independent prognostic factor of six-week mortality in older patients hospitalized for an acute condition? Qual Life Res. 2016;25(9):2335-40.

14. Downar J, Goldman R, Pinto R, Englesakis M, Adhikari NK. The "surprise question" for predicting death in seriously ill patients: a systematic review and meta-analysis. CMAJ. 2017;189(13):E484-e93.

15. White N, Kupeli N, Vickerstaff V, Stone P. How accurate is the 'Surprise Question' at identifying patients at the end of life? A systematic review and meta-analysis. BMC Med. 2017;15(1):139.

16. Buurman BM, van Munster BC, Korevaar JC, Abu-Hanna A, Levi M, de Rooij SE. Prognostication in acutely admitted older patients by nurses and physicians. J Gen Intern Med. 2008;23(11):1883-9.

17. Hamano J, Morita T, Inoue S, Ikenaga M, Matsumoto Y, Sekine R, et al. Surprise Questions for Survival Prediction in Patients With Advanced Cancer: A Multicenter Prospective Cohort Study. Oncologist. 2015;20(7):839-44.

18. Charlson ME, Pompei P, Ales KL, MacKenzie CR. A new method of classifying prognostic comorbidity in longitudinal studies: development and validation. Journal of chronic diseases. 1987;40(5):373-83.

19. Katz S, Ford AB, Moskowitz RW, Jackson BA, Jaffe MW. Studies off illness in the aged. The index of ADL: a standardized measure of biological and psychosocial function. JAMA. 1963;185:914-9.

20. World Health O. ICD-10 : international statistical classification of diseases and related health problems : tenth revision / Vol. 2, Instruction manual. 2nd ed. ed. Geneva: WHO; 2004.

21. Simundic AM. Measures of Diagnostic Accuracy: Basic Definitions. Ejifcc. 2009;19(4):203-11.

22. DeLong ER, DeLong DM, Clarke-Pearson DL. Comparing the areas under two or more correlated receiver operating characteristic curves: a nonparametric approach. Biometrics. 1988;44(3):837-45. 
23. Hallgren KA. Computing Inter-Rater Reliability for Observational Data: An Overview and Tutorial. Tutor Quant Methods Psychol. 2012;8(1):23-34.

24. Cicchetti DV. Guidelines, criteria, and rules of thumb for evaluating normed and standardized assessment instruments in psychology. Psychological Assessment. 1994;6(4):284-90.

25. Quinten VM, van Meurs M, Wolffensperger AE, Ter Maaten JC, Ligtenberg JJM. Sepsis patients in the emergency department: stratification using the Clinical Impression Score, Predisposition, Infection, Response and Organ dysfunction score or quick Sequential Organ Failure Assessment score? Eur J Emerg Med. 2018;25(5):328-35. 


\section{Supplemental data}

See additional files chapter 2 . 



\section{Chapter 4}

Concerns of older patients and their caregivers in the emergency department

N. Zelis, S.E. Huisman, A.N. Mauritz, J. Buijs, P.W. de Leeuw, P.M. Stassen PLoS One 2020;15(7):e0235708 


\section{Abstract}

\section{Background}

Older emergency department (ED) patients often have complex problems and severe illnesses with a high risk of adverse outcomes. It is likely that these older patients are troubled with concerns, which might reflect their preferences and needs concerning medical care. However, data regarding this topic are lacking.

\section{Methods}

This study is a sub study of a prospective, multicenter, observational cohort study among older medical ED patients ( $\geq 65$ years). Patients or their caregivers were asked about their illness-related concerns during the first stage of the ED visit using a questionnaire. All concerns were categorized into 10 categories, and differences between patients and caregivers, and between age groups were analyzed. Odds Ratios were calculated to determine the association of the concerns for different adverse outcomes.

\section{Results}

Most of the 594 included patients (or their caregivers) were concerned (88\%) about some aspects of their illness or their need for medical care. The most often reported concerns were about the severity of disease (43.6\%), functional decline (9.4\%) and dying (5.6\%). Caregivers were more frequently concerned than patients $(P<0.001)$ especially regarding the severity of disease ( 50.5 vs $39.6 \%, P=0.016)$ and cognitive decline (10.8 vs. $0.3 \%, \mathrm{P}<0.001)$. We found no difference between age groups. The concern about dying was associated with 30-day mortality (OR 2.89; 95\% Cl: 1.24-6.70) and the composite endpoint (intensive- or medium care admission, length of hospital stay $>7$ days, loss of independent living and unplanned readmission within 30 days) (OR 2.32; 95\% $\mathrm{Cl}: 1.12-4.82$ ). In addition, unspecified concerns were associated with mortality (OR 1.88; 95\% Cl: 1.09-3.22).

\section{Conclusion}

The majority of older patients and especially their caregivers are concerned about their medical condition or need for medical care when they visit the ED. These concerns are associated with adverse outcomes and most likely reflect their needs regarding medical care. More attention should be paid to these concerns because they may offer opportunities to reduce anxiety and provide care that is adjusted to their needs. 


\section{Introduction}

In all likelihood, older patients ( $\geq 65$ years) who have been referred to an emergency department (ED) experience great concerns regarding their condition and their need for medical care. This is not surprising as these patients often present with complex problems and severe illnesses with a high risk of an adverse outcome, including the loss of independence and death. ${ }^{1-3}$

Nevertheless, only a few studies have reported on the concerns of ED patients ${ }^{4-7}$, but these focused mainly on their overall experience during the ED visit and not explicitly on their concerns. In addition, these studies were performed in younger patients and/or in small populations. ${ }^{5,6,8,9}$ Only in one study, patients were explicitly asked about their concerns, but this was done in an out-patient setting. ${ }^{6}$ Since many patients feel inhibited to talk about their feelings to the ED staff ${ }^{8,10}$, it is likely that their concerns have not been completely exposed in these studies. Recently, we performed the RISE UP study to find predictors of adverse outcomes in older ED patients $^{11}$, and found that the severity of concerns in patients and caregivers is predictive of adverse outcomes. ${ }^{12}$ It may, therefore, also be possible that specific concerns are predictive of that particular outcome.

We specifically explored the nature of the concerns and their prognostic value in the RISE UP study cohort consisting of older ED patients. We hypothesized that older ED patients or their caregivers experience a broad spectrum of concerns regarding their medical condition and medical care, which may be associated with adverse clinical outcomes. We further focused on the types of concerns of patients, caregivers and different age groups.

\section{Materials and methods}

\section{Study design, setting and study population}

This study is part of the RISE UP study, a prospective multicenter observational cohort study, aiming to identify predictors for adverse outcome in older medical ED patients. In short, this study was conducted at the EDs in Zuyderland Medical Center (MC) and Maastricht University Medical Center+ (MUMC+) in The Netherlands from July 2016 until February 2017. Older ED patients ( $\geq 65$ years) treated by internists or gastroenterologists were eligible for inclusion after informed consent was obtained 
from the patient or legal representative. The study protocol was approved by the medical ethics committees of Zuyderland MC and MUMC+ (NL55867.096.15) and published online. ${ }^{11}$ This study was reported in line with the STROBE (STrengthening the Reporting of OBservational studies in Epidemiology) guidelines. ${ }^{13}$

\section{Data collection}

In the RISE UP study, all participants (patients or caregivers) received a questionnaire, which was filled out immediately after admission to the ED, before history taking and physical examination by the physician. The questionnaire contained questions about their concerns, disease perception and self-rated health. Results of this questionnaire, regarding the predictive value of clinical intuition for adverse outcomes, have been published online. ${ }^{12}$ For the current study, we focused on the categorical question: 'Are you concerned about your (his/her) condition?' and the open question 'If you are concerned about your (his/her) condition, what are you concerned about?'. All the answers to the categorical and open questions were entered in SPSS. Subsequently all answers were categorized independently by two researchers into the following ten categories: no concern; severity of disease; functional decline; cognitive decline; dying; relatives; diagnostic procedures or treatment; not being acknowledged; miscellaneous and not further specified. As some patients/caregivers mentioned more than one illness-related concern, we included and analyzed the two first mentioned concerns. In case of disagreement between the two researchers, a third researcher decided on the issue.

Additionally, we retrieved from the medical records the following data to phenotype our population: demographics, living situation, comorbidities (quantified according to the Charlson Comorbidity Index $(\mathrm{CCl})^{14}$ ) and cognitive function. Functional status was assessed using a questionnaire to determine the Katz Activities of Daily Living (ADL) index score ${ }^{15}$, which was filled out for all hospitalized patients. The main reason for the ED-visit was categorized according to the International Classification of Diseases (ICD)-10. ${ }^{16}$

\section{Outcome measures}

The primary outcome of this study was 30-day all-cause mortality. The secondary endpoints were a) a composite endpoint consisting of intensive or medium care unit (ICU/MCU) admission, prolonged length of hospital stay (LOS; >7 days), loss of independent living and unplanned readmission within 30 days after discharge, and $b$ ) 
loss of independent living. All patients were followed up for at least 30 days to obtain outcomes.

To assess the association of the illness-related concern categories "no concern", "severity of disease", "dying" and "not further specified" with adverse outcomes, we used 30-day mortality and the secondary composite outcome. For the two concern categories "functional and cognitive decline", we used the secondary loss of independent living outcome. We chose to evaluate the association between these six concern categories and adverse outcome because we hypothesized that these specific categories would be associated with the outcome measures.

\section{Statistical analysis}

The sample size of this study was based on that of the RISE UP study. We performed descriptive analyses of the baseline characteristics, types of concerns and outcome measures. For the analysis regarding differences of concerns between patients and caregivers, we excluded questionnaires in which it was unclear whether the patient or the caregiver filled out the form. To study the differences in concerns between age groups, the study population was divided into two groups: $65-79$ years and $\geq 80$ years of age. Differences in concerns between patients and caregivers and between age groups were analyzed using the Chi-square or Fisher's Exact tests, when appropriate. We used univariable logistic regression analyses to determine the predictive value of specific concerns for the outcomes. Odds ratios (ORs) with $95 \%$ confidence intervals (Cls) were calculated. All analyses were performed using IBM SPSS Statistics for Windows, Version 24.0 (IBM Corp., Armonk, N.Y., USA). P-values <0.05 were considered statistically significant.

\section{Results}

\section{Study population and characteristics}

During the study period 603 patients were included. Detailed information regarding the patient selection was described in our previous article. ${ }^{12}$ Questionnaires were missing in 9 patients, so 594 patients were included in the final analysis. The median age of the patients was 79 years (IQR 73-85) and 52\% were male (Table 4.1). Most patients were community-dwelling (86.7\%) with a median Katz-ADL score of 0 , which means that most of the patients were independent with respect to ADL. Cognitive impairment (including delirium) was present in 164 (29.0\%) patients. 
Table 4.1 Patient characteristics.

\begin{tabular}{lc}
\hline & All patients \\
& $\mathrm{N}=\mathbf{5 9 4}$ \\
\hline Demographics & $79(73-85)$ \\
Median (IQR) Age, years & $309(52.0)$ \\
Male, $\mathrm{n}, \%$ & $515(86.7)$ \\
Community dwelling, $\mathrm{n}(\%)$ & \\
Comorbidity and functional status & $2(1-3)$ \\
Median (IQR) CCI score & $0(0-2)$ \\
Median (IQR) Katz-ADL index score ${ }^{\mathrm{a}}$ & $164(29.0)$ \\
Dementia, mild cognitive impairment or delirium $\mathrm{n}(\%)^{\mathrm{b}}$ & \\
Reason for ED-visit (ICD-10), $\mathrm{n}(\%)$ & $174(29.3)$ \\
Infectious and parasitic disease & $155(26.1)$ \\
Diseases of the digestive system & $54(9.1)$ \\
Diseases of the circulatory system & $50(8.4)$ \\
Neoplasms & $31(5.2)$ \\
Endocrine, nutritional and metabolic diseases & $30(5.1)$ \\
Diseases of the respiratory system & $27(4.5)$ \\
Diseases of blood and blood-forming organs & $27(4.5)$ \\
Diseases of the genitourinary system & $46(7.7)$ \\
Miscellaneous & \\
\hline
\end{tabular}

SD, Standard Deviation; CCl, Charlson Comorbidity Index; ADL, Activities of Daily Living; ICD-10, International Classification of Diseases-10. ${ }^{a}$ Katz-ADL index score determined in all hospitalized patients $(n=472)$; ${ }^{b}$ Denominator: $n=566$.

\section{Outcomes}

Of the 594 patients, $64(10.8 \%)$ died within 30 days after the ED visit (primary outcome). In total, 262 (44.1\%) met the secondary composite outcome (ICU/MCU admission, prolonged LOS, loss of independent living and unplanned readmission within 30 days after discharge). The secondary loss of independent living outcome occurred in $82(14.8 \%)$ patients. Follow up was complete for all patients with regard to all outcome measures.

\section{Concerns of patients and caregivers}

The questionnaires were mostly answered by patients $(51.9 \%)$, in $32.7 \%$ by the caregivers, while in $15.5 \%$, it was unclear who filled out the form. A majority of respondents ( $n=523,88.0 \%$ ) expressed at least one illness-related concern. In 363 (69.4\%) of the questionnaires, the concerns were further specified. An illustration of the answers is presented in Table S4.1. Patients and caregivers were mostly concerned about the severity of disease $(n=259 ; 43.6 \%)$, functional decline ( $n=56$; $9.4 \%$ ) and dying ( $n=33 ; 5.6 \%$; Figure 4.1). 
Patients in who the respondent was concerned during the ED visit were younger (median 79 vs. 81 years, p-value 0.03) and their reason for the ED visit was more often because of cancer (9.4 vs. 1.4\%) compared to patients in which the patient or caregiver was not concerned (Table S4.2).

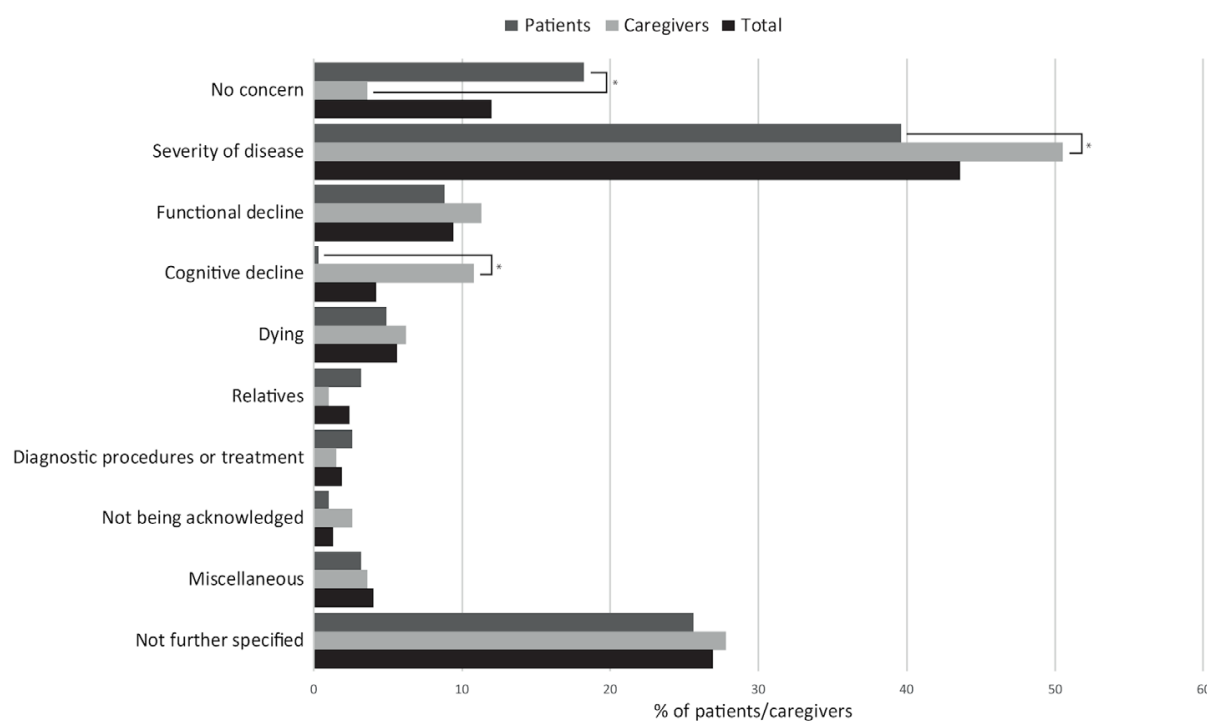

Figure 4.1 Concerns of patients and caregivers. This figure presents the type of illness related concerns of patients and caregivers in the first stage of the ED visit. Significant differences between patients and caregivers ( $p$-value $<0.05$ ) are marked with an asterisk*.

Differences in concerns between patients and caregivers and age groups

Patients were less often concerned about their medical condition compared to their caregivers $(p<0.001$, Figure 4.1). In addition, patients were less often concerned about the severity of their disease (39.6\%) and about cognitive decline $(0.3 \%)$ compared to the caregivers $(50.5 \%, p=0.016$ and $10.8 \%, p<0.001$, resp.).

When we compared the two age groups, we found no significant differences with respect to these items (Table 4.2). 
Table 4.2 Concerns in different age groups ${ }^{a}$.

\begin{tabular}{lcc}
\hline Type of concern & \multicolumn{2}{c}{ Age (years) } \\
& $\mathbf{6 5 - 7 9}$ & $\begin{array}{c}\geq \mathbf{8 0} \\
\text { (n=239) }\end{array}$ \\
\hline No concern & $31(9.8)$ & $40(14.3)$ \\
Severity of disease & $148(47.0)$ & $111(39.8)$ \\
Functional decline & $24(7.6)$ & $32(11.5)$ \\
Cognitive decline & $11(3.5)$ & $14(5.0)$ \\
Dying & $15(4.8)$ & $18(6.5)$ \\
Relatives & $9(2.9)$ & $5(1.8)$ \\
Diagnostic procedures or treatment & $5(1.6)$ & $6(2.2)$ \\
Not being acknowledged & $3(1.0)$ & $5(1.8)$ \\
Miscellaneous & $12(3.8)$ & $12(4.3)$ \\
Not further specified & $92(29.2)$ & $68(24.4)$ \\
\hline
\end{tabular}

${ }^{\mathrm{a}}$ No significant differences found between the age groups.

\section{Types of concerns and their association with outcome}

We found that if a patient or caregiver was concerned about dying, this concern was associated with 30-day mortality (OR 2.89; $95 \% \mathrm{Cl}$ : 1.24-6.70, Table 4.3). Likewise, unspecified concerns were associated with mortality (OR 1.88; 95\% Cl: 1.09-3.22). In addition, the concern of dying was associated (OR $2.32 ; 95 \% \mathrm{Cl}: 1.12-4.82$ ) with the secondary composite outcome (ICU/MCU admission, prolonged LOS, loss of independent living and unplanned readmission) as well. Concerns about functional or cognitive decline were not associated with the secondary loss of independent living outcome.

Table 4.3 Logistic regression analysis of type of concerns for different outcome measures.

\begin{tabular}{lcccccr}
\hline Type of concern & \multicolumn{2}{c}{ Mortality } & \multicolumn{2}{c}{ Composite endpoint $^{\text {a }}$} & \multicolumn{2}{c}{ Loss of independent living } \\
& OR (95\% Cl) & P-value & OR (95\% Cl) & P-value & OR (95\% Cl) & P-value \\
\hline No concern $(n=71)$ & $0.89(0.39-2.05)$ & 0.791 & $0.61(0.36-1.03)$ & 0.064 & - & \\
Severity of disease $(n=259)$ & $0.65(0.38-1.12)$ & 0.117 & $0.91(0.66-1.27)$ & 0.589 & - & \\
Functional decline $(n=56)$ & - & - & - & - & $1.08(0.49-2.39)$ & 0.852 \\
Cognitive decline $(n=25)$ & - & - & - & $2.11(0.81-5.53)$ & 0.127 \\
Dying $(n=33)$ & $2.89(1.24-6.70)$ & 0.014 & $2.32(1.12-4.82)$ & 0.023 & - & \\
Not specified $(n=160)$ & $1.88(1.09-3.22)$ & 0.022 & $0.98(0.68-1.41)$ & 0.915 & - & \\
\hline
\end{tabular}

$\mathrm{Cl}$, confidence interval; OR, Odds ratio. ${ }^{\mathrm{a} C o m p o s i t e ~ e n d p o i n t: ~ I C U / M C U ~ a d m i s s i o n, ~ p r o l o n g e d ~ L O S, ~ l o s s ~ o f ~}$ independent living and unplanned readmission within 30 days. 


\section{Discussion}

In this prospective multicenter study we showed that most older ED patients and their caregivers (88\%) expressed a variety of concerns related to the patient's medical condition or to the medical care, when they visit the ED. Patients and caregivers were particularly concerned about the severity of disease, functional decline and dying. Caregivers were more frequently concerned than patients, especially regarding the severity of disease and cognitive decline. Their concerns were also associated with adverse outcomes. This is illustrated by the finding that when there was a concern about dying, patients were almost three times more likely to die within 30 days.

To the best of our knowledge, our study is the first to explicitly ask older patients (or their caregivers) about their illness-related concerns during an ED visit using an open question. We found that most concerns were about the severity of disease, functional decline and dying. We focused on concerns upon arrival at the ED because it is possible that concerns dissipate after clarification of the medical problem and prognosis. To date, information regarding this topic is lacking. Most studies focused on the experience of patients during the ED visit (e.g. waiting times and communication by ED staff), and/or were performed in younger patients, smaller populations or other settings. ${ }^{4,5,7-9}$ In only one Swedish study patients were explicitly interviewed about their concerns when visiting an outpatient clinic. ${ }^{6}$ Their concerns were either practical or about their disease. Similar to our findings, they found that many patients could not specify the reason for their concern. A small Dutch qualitative study on the experience of patients during their ED stay ${ }^{5}$ showed that most patients were concerned (144 concerns in 16 observed patients) and that many concerns were present early during the ED visit. However, in this Dutch study, patients were not explicitly asked about their concerns, and therefore, it is possible that not all concerns were disclosed. In addition, an American study ${ }^{17}$, showed that many patients (25\%) visiting the ED for suspected acute coronary syndrome were afraid to die. However, in this American study, patients answered closed questions in a questionnaire regarding several established concerns and therefore, it is possible that not all concerns were retrieved. Despite the differences in setting and design, our study and those of others show that most patients are concerned about their medical condition or need of medical care. Since patients often do not discuss their concerns with nurses or physicians $s^{8,10}$, they probably will not spontaneously discuss their concerns with researchers either. Therefore, it is likely that if patients (or caregivers) are explicitly asked about their concerns using an open question, they will reveal the 
(true nature of their) concerns, which in turn probably reflect their needs and preferences regarding medical care.

Interestingly, we found differences in concerns between patients and caregivers. Patients were less concerned with respect to disease severity or cognitive decline compared to their caregivers. The difference we found may be due to the nature or severity of disease for which the patients visited the ED, by the inability to assess the severity of their disease, by possible impairment in cognitive function or by the belief that one's life is final or complete. In addition, it is possible that the questionnaires of patients with more severe underlying illness and/or cognitive impairment were more frequently filled out by caregivers. In line with our findings is that other studies show that the perception of a patients' health differs between patients and caregivers. ${ }^{18-20}$ Therefore, we think it is important to keep in mind that the concerns of patients and caregivers can be different as well as the needs of patients and caregivers during an ED visit.

Some of the concerns of patients and/or their caregivers were associated with adverse outcomes. When a patient/caregiver was concerned about dying, this concern was associated with 30-day mortality (OR 2.89) and the secondary composite outcome (OR 2.32). Moreover, unspecified concerns were associated with a higher risk of mortality (OR 1.88). This finding probably means that, for patients or caregivers, it is sometimes difficult to specify their concern of dying. Since many patients are concerned about dying, it may be important to ask our patients or caregivers about this concern. This could also provide an opportunity to talk about the patient's goals, their preferences or end-of-life care, an important yet not often discussed topic in ED care. ${ }^{21,22}$ By discussing these subjects, one could avoid unwanted treatments and could improve quality of care.

Surprisingly, the concern about functional or cognitive decline was not associated with loss of independent living. This is in line with an UK study, showing that the expected need for additional care support by patients was not predictive of their actual need. An explanation for this finding is that in an early stage of the ED visit patients or caregivers are unaware of the nature and severity of the medical problem and its impact on the patient's functioning. Another explanation might be that patients who are at risk of functional or cognitive decline are not aware of this. Since concerns of patients and caregivers are associated with adverse outcomes it is important to elucidate these concerns. 


\section{Implications for clinical practice}

We would like to address a few possible implications for clinical practice. First, we think it is very important to explicitly ask patients about their concerns when visiting the ED, since most patients are concerned but may be reserved to talk about these concerns to the staff. We think an open question is preferable because this offers an opportunity to freely communicate on all concerns. Second, because concerns are clearly associated with adverse outcomes, it is important to communicate with patients and caregivers about concerns regarding their prognosis and preferences regarding end-of-life care.

\section{Strengths, limitations and future perspectives}

To date, this is the largest study regarding the concerns of older ED patients. Due to the inclusion of both patients and their caregivers a quite extensive study population could be analyzed, which strengthens our conclusion. A limitation of our study is that it was restricted to medical (internal medicine/gastroenterology) patients. It is not clear whether our results can be extrapolated to other patients visiting the ED. However, medical patients often present with complex medical problems, which are possibly accompanied by many different concerns. Another limitation is that we used questionnaires, while an open interview may have yielded more detailed information and could have explored other types of concerns. However, our study has shown that a qualitative study that explores the concerns of older ED patients is worth designing. In addition, as we did not control for confounders of adverse outcome we cannot conclude on whether the concerns of older patients are independently associated with the outcomes. This aspect of the concerns was not the scope of our study. Last, many patients or caregivers were concerned about losing independency. It is possible that the way societies take care of older patients influences this concern. In the Netherlands, if an older patient is in need of extensive homecare support, they will often move to a nursing home. In other cultures, in these circumstances, patients move in with their children, which may result in other kinds of concerns.

Future studies regarding this topic should focus on the concerns in the overall ED population, in other countries and use interviews in order to specify the types of concerns and explore the needs that ED patients, and especially older ED patients, have. These studies may provide insight in how to address and reduce these concerns and lead to more understanding and more empathic contacts with the health care workers in the ED. Other studies show that effective communication, pain management and involvement of caregivers helps reducing anxiety in ED patients. ${ }^{4,8}$ 
Therefore, the importance of early communication with patients about the nature of their medical problem, its' impact on functioning and possible treatment options together with early involvement of caregivers in reducing concerns, anxiety and worries is another important subject to study.

\section{Conclusions}

Older patients and particularly their caregivers are concerned about their medical condition or need for medical care when they visit the ED. These concerns are often justified since they are associated with serious adverse outcomes. We can easily clarify these concerns by asking them just one open question: 'What are you concerned about?'. We think that it is important to find a moment to ask this question, because only when we are aware of the type of concerns or needs of our patients/caregivers, we are able to find ways to reduce these concerns and meet the needs in order to improve their experience and/or outcome and to make ED care more person-centered.

\section{Acknowledgements}

We thank all of the patients and caregivers for their participation in this study. We also would like to thank all of the medical staff of Zuyderland MC and MUMC+ who contributed to this study. In addition, we would like to thank LG Lambriks for the design of the figure and LIJ Kuijpers for her help in collecting the data. 


\section{References}

1. Samaras N, Chevalley T, Samaras D, Gold G. Older patients in the emergency department: a review. Ann Emerg Med. 2010;56(3):261-9.

2. Aminzadeh F, Dalziel WB. Older adults in the emergency department: a systematic review of patterns of use, adverse outcomes, and effectiveness of interventions. Ann Emerg Med. 2002;39(3):238-47.

3. Schnitker L, Martin-Khan M, Beattie E, Gray L. Negative health outcomes and adverse events in older people attending emergency departments: A systematic review. Austr Emerg Nurs J. 2011;14(3): 141-62.

4. Shankar KN, Bhatia BK, Schuur JD. Toward patient-centered care: a systematic review of older adults' views of quality emergency care. Ann Emerg Med. 2014;63(5):529-50.e1.

5. Olthuis G, Prins C, Smits MJ, van de Pas H, Bierens J, Baart A. Matters of concern: a qualitative study of emergency care from the perspective of patients. Ann Emerg Med. 2014;63(3):311-9.e2.

6. Andersson-Segesten K, Erichsen M, Westerlund A, Ojerskog I. Patients' fears, worries, and concerns when visiting an out-patient clinic. Scand J Prim Health Care. 1989;7(4):197-201.

7. Nairn S, Whotton E, Marshal C, Roberts M, Swann G. The patient experience in emergency departments: a review of the literature. Accid Emerg Nurs. 2004;12(3):159-65.

8. Baraff $\amalg$, Bernstein E, Bradley K, Franken C, Gerson LW, Hannegan SR, et al. Perceptions of emergency care by the elderly: results of multicenter focus group interviews. Ann Emerg Med. 1992;21(7):814-8.

9. Kihlgren AL, Nilsson M, Skovdahl K, Palmblad B, Wimo A. Older patients awaiting emergency department treatment. Scand J Caring Sci. 2004;18(2):169-76.

10. Lim C, Berry ABL, Hirsch T, Hartzler AL, Wagner EH, Ludman E, et al. "It just seems outside my health": How Patients with Chronic Conditions Perceive Communication Boundaries with Providers. DIS Designing Interactive Systems (Conference). 2016;2016:1172-84..

11. Zelis N, Buijs J, de Leeuw PW, van Kuijk SMJ, Stassen PM. Study protocol for a multicentre prospective cohort study to identify predictors of adverse outcome in older medical emergency department patients (the Risk Stratification in the Emergency Department in Acutely III Older Patients (RISE UP) study). BMC Geriatr. 2019;19(1):65.

12. Zelis N, Mauritz AN, Kuijpers LIJ, Buijs J, de Leeuw PW, Stassen PM. Short-term mortality in older medical emergency patients can be predicted using clinical intuition: A prospective study. PloS One. 2019;14(1):e0208741

13. von Elm E, Altman DG, Egger M, Pocock SJ, Gøtzsche PC, Vandenbroucke JP. The Strengthening the Reporting of Observational Studies in Epidemiology (STROBE) statement: guidelines for reporting observational studies. J Clin Epidemiol. 2008;61(4):344-9.

14. Charlson ME, Pompei P, Ales KL, MacKenzie CR. A new method of classifying prognostic comorbidity in longitudinal studies: development and validation. J Chronic Dis. 1987;40(5):373-83.

15. Katz S, Ford AB, Moskowitz RW, Jackson BA, Jaffe MW. Studies of illness in the aged. The Index of ADL: a standardized measure of biological and psychosocial function. JAMA. 1963;185:914-9.

16. Organisation WH. International Statistical Classification Of Diseases and Related Health Problems, 10th Revision. Version 2016 ed.

17. White M, Edmondson D, Umland R, Sanchez G, Chang BP. Patient perceptions of stress during evaluation for ACS in the ED. The American journal of emergency medicine. 2017;35(2):351-2. Epub 2016/11/09. doi: 10.1016/j.ajem.2016.10.053. PubMed PMID: 27823939; PubMed Central PMCID: PMCPMC5728103.

18. Hsu T, Loscalzo M, Ramani R, Forman S, Popplewell L, Clark K, et al. Are Disagreements in Caregiver and Patient Assessment of Patient Health Associated with Increased Caregiver Burden in Caregivers of Older Adults with Cancer? Oncologist. 2017;22(11):1383-91.

19. Neumann PJ, Araki SS, Gutterman EM. The use of proxy respondents in studies of older adults: lessons, challenges, and opportunities. J Am Geriatr Soc. 2000;48(12):1646-54.

20. Yasuda N, Zimmerman S, Hawkes WG, Gruber-Baldini AL, Hebel JR, Magaziner J. Concordance of proxy-perceived change and measured change in multiple domains of function in older persons. J Am Geriatr Soc. 2004;52(7):1157-62. 
21. Barry MJ, Edgman-Levitan S. Shared Decision Making - The Pinnacle of Patient-Centered Care. N Engl J Med. 2012;366(9):780-1.

22. Committee on Approaching Death: Addressing Key End of Life I. Dying in America: Improving Quality and Honoring Individual Preferences Near the End of Life: National Academies Press (US); 2015. 


\section{Supplemental data}

Table S4.1 Concerns of patients and caregivers.*

\begin{tabular}{|c|c|}
\hline Concerns & $\begin{array}{c}\text { Total } \\
\mathrm{N}=363\end{array}$ \\
\hline About (the severity of) the condition or illness & $32^{\mathrm{a}}$ \\
\hline Because of the uncertainty what causes the problems/illness & $29^{a}$ \\
\hline About dying & $21^{\mathrm{d}}$ \\
\hline Because of the uncertainty regarding my condition & $20^{\mathrm{a}}$ \\
\hline About the (declining) health and functioning & $18^{\mathrm{a}, \mathrm{b}}$ \\
\hline Because of the pain & $14^{\mathrm{a}}$ \\
\hline About not getting better & $12^{\mathrm{a}}$ \\
\hline About decline in cognitive and physical functioning & $10^{b, c}$ \\
\hline Whether everything will turn out fine or not & $9^{a}$ \\
\hline About cognitive problems or cognitive functioning & $9^{c}$ \\
\hline Because he/she/I was never this ill before & $8^{\mathrm{a}}$ \\
\hline About age in relation to health condition or illness & $8^{a}$ \\
\hline About having cancer & $8^{a}$ \\
\hline Afraid that it is something serious & $8^{a}$ \\
\hline About heart disease & $8^{a}$ \\
\hline About losing independence and/or decline in physical functioning & $8^{\mathrm{b}}$ \\
\hline Uncertainty (about the future) & $8^{\text {h }}$ \\
\hline Because this is the first ED visit and/or it all happened so quickly & $8^{\text {h }}$ \\
\hline About shortness of breath & $7^{\mathrm{a}}$ \\
\hline About the test results & $6^{\mathrm{a}}$ \\
\hline About being unable to eat or drink and/or the risk for dehydration & $6^{a}$ \\
\hline Because of the recurrent symptoms & $6^{a}$ \\
\hline About severity of disease and cognitive functioning & $6^{\mathrm{a}, \mathrm{c}}$ \\
\hline About the bleeding/ loss of blood & $5^{a}$ \\
\hline About my (transplant) kidney(s) & $5^{a}$ \\
\hline About losing a loved one & $5^{d, e}$ \\
\hline About the high fever & $4^{a}$ \\
\hline Because the therapy is not working & $4^{a, f}$ \\
\hline About severity of disease and functional decline & $4^{\mathrm{a}, \mathrm{b}}$ \\
\hline About quality of life & $4^{b}$ \\
\hline About my loved ones & $4^{\mathrm{e}}$ \\
\hline About severity of disease and dying & $3^{\mathrm{a}, \mathrm{d}}$ \\
\hline About loss of independence and afraid of dying & $3^{\mathrm{b}, \mathrm{d}}$ \\
\hline About the Parkinson's disease & $3^{\mathrm{a}}$ \\
\hline Because it takes a long time (to recover) & $3^{\mathrm{a}}$ \\
\hline Afraid she cannot take care of her loved ones anymore & $3^{\mathrm{b}, \mathrm{e}}$ \\
\hline Afraid of diagnostics or therapy (including surgery) & $3^{f}$ \\
\hline About lung disease & $2^{\mathrm{a}}$ \\
\hline About being hospitalized for this illness & $2^{a, f}$ \\
\hline Because my loved one is worried & $2^{\text {h }}$ \\
\hline Because problems are getting worse and the patient cannot specify the symptoms or whishes & $2^{\mathrm{a}, \mathrm{g}}$ \\
\hline Because the patient cannot accept the loss of independence & $2^{\mathrm{b}, \mathrm{g}}$ \\
\hline About feeling miserable. About what needs to be arranged. & $1^{\mathrm{a}, \mathrm{b}}$ \\
\hline About high blood pressure & $1^{\mathrm{a}}$ \\
\hline About my diabetes and COPD & $1^{\mathrm{a}}$ \\
\hline
\end{tabular}


Table S4.1 continued

\begin{tabular}{|c|c|}
\hline Concerns & $\begin{array}{c}\text { Total } \\
\mathrm{N}=363\end{array}$ \\
\hline About osteoporosis & $1^{\mathrm{a}}$ \\
\hline About the mistakes and diseases in the past & $1^{\mathrm{a}, \mathrm{g}}$ \\
\hline About the blood sugar being too low & $1^{\mathrm{a}}$ \\
\hline Afraid that I have something in my head & $1^{\mathrm{a}}$ \\
\hline Because she has a lot of medical problems & $1^{\mathrm{a}}$ \\
\hline Because the physician said she is pessimistic about the situation & $1^{\mathrm{a}}$ \\
\hline Because there's no treatment possible anymore & $1^{a}$ \\
\hline I am a little bit worried & $1^{\mathrm{a}}$ \\
\hline I was frightened by the telephone call of the general practitioner & $1^{\mathrm{a}}$ \\
\hline I'm afraid something is wrong with my pancreas & $1^{\mathrm{a}}$ \\
\hline It is not that bad, it will be alright & $1^{\mathrm{a}}$ \\
\hline This medical problem was found by population screening & $1^{\mathrm{a}}$ \\
\hline Worried about cirrhosis of the liver & $1^{\mathrm{a}}$ \\
\hline Because she only recently started living on her own after her husband died & $1^{\mathrm{b}}$ \\
\hline Worried about work absence because of the severity of this illness & $1^{\mathrm{a}, \mathrm{b}}$ \\
\hline Because he is not that old and still had around 10 years to enjoy life & $1^{d}$ \\
\hline About the loss of my son 6 months ago & $1^{e}$ \\
\hline Worried about real estate and relatives & $1^{\mathrm{b}, \mathrm{e}}$ \\
\hline About the completion of chemotherapy & $1^{f}$ \\
\hline Worried about this visit in relation to the chemotherapy & $1^{\mathrm{f,h}}$ \\
\hline Because it is unfortunate that my father cannot specify his wishes & $1^{\mathrm{g}}$ \\
\hline Whether I am being heard/believed & $1^{\mathrm{g}}$ \\
\hline Whether I am in good hands and not only seen by interns & $1^{\mathrm{g}}$ \\
\hline Because he is quickly worried about things & $1^{\mathrm{h}}$ \\
\hline Because my mom doesn't know how to continue & $1^{\mathrm{h}}$ \\
\hline About being desperate & $1^{\mathrm{h}}$ \\
\hline I am worried that something will happen at home & $2^{h}$ \\
\hline
\end{tabular}

* Most of the concerns shown in the table are not the literal answers provided by patients or caregivers. Similar answers were combined in order to concise the table. The superscript characters ' $a$ ' to ' $h$ ' refer to the category in which we categorized the concerns: ${ }^{a}$ Severity of disease, ${ }^{b}$ Functional decline, ${ }^{\mathrm{c}}$ Cognitive decline, ${ }^{\mathrm{d}}$ Dying, ${ }^{\mathrm{e}}$ Relatives, ${ }^{\mathrm{f}}$ Diagnostic procedures or treatment, ${ }^{\mathrm{g}}$ Not being acknowledged, ${ }^{\mathrm{h}}$ Miscallaneous. 
Table S4.2 Overview of patients' characteristics when a patient/caregiver was either concerned or was not concerned.

\begin{tabular}{|c|c|c|}
\hline Patient characteristic & $\begin{array}{c}\text { Patient/caregiver } \\
\text { Concerned } \\
\mathrm{N}=523\end{array}$ & $\begin{array}{c}\text { Patient/Caregiver } \\
\text { Not Concerned } \\
\mathbf{N}=71\end{array}$ \\
\hline \multicolumn{3}{|l|}{ Demographics } \\
\hline Median (IQR) Age, years & $79(73-85)$ & $81(75-86)^{c}$ \\
\hline Male, $n, \%$ & $269(51.4)$ & $40(56.3)$ \\
\hline Community dwelling, $\mathrm{n}(\%)$ & $452(86.4)$ & $63(88.7)$ \\
\hline \multicolumn{3}{|l|}{ Comorbidity and functional status } \\
\hline Median (IQR) CCI score & $2(1-3)$ & $2(1-3)$ \\
\hline Median (IQR) Katz-ADL index score ${ }^{a}$ & $0(0-2)$ & $0(0-2)$ \\
\hline Dementia, mild cognitive impairment or delirium, $n(\%)^{b}$ & $142(28.6)$ & $22(31.4)$ \\
\hline \multicolumn{3}{|l|}{ Reason for ED-visit (ICD-10), n (\%) } \\
\hline Infectious and parasitic disease & $157(30.0)$ & $17(23.9)$ \\
\hline Diseases of the digestive system & $132(25.2)$ & $23(32.4)$ \\
\hline Diseases of the circulatory system & $48(9.2)$ & $6(8.5)$ \\
\hline Neoplasms & $49(9.4)$ & $1(1.4)$ \\
\hline Endocrine, nutritional and metabolic diseases & $24(4.6)$ & $7(9.9)$ \\
\hline Diseases of the respiratory system & $26(5.0)$ & $4(5.6)$ \\
\hline Diseases of blood and blood-forming organs & $23(4.4)$ & $4(5.6)$ \\
\hline Diseases of the genitourinary system & $24(4.6)$ & $3(4.2)$ \\
\hline Miscellaneous & $40(7.6)$ & $6(8.5)$ \\
\hline
\end{tabular}

SD, Standard Deviation; CCl, Charlson Comorbidity Index; ADL, Activities of Daily Living; ICD-10, International Classification of Diseases-10. ${ }^{a}$ Katz-ADL index score determined in all hospitalized patients ( $n=471$ ); ${ }^{b}$ Denominator count: $n=496$ and $n=70$, respectively; ${ }^{c}$ significantly different with $P$-value $<0.05$. 



\section{Chapter 5}

\section{A new simplified model for predicting 30-day mortality in older medical emergency department patients: the RISE UP score}

N. Zelis, J. Buijs, P.W. de Leeuw, S.M.J. van Kuijk, P.M. Stassen

European Journal of Internal Medicine 2020;77:36-43 


\section{Abstract}

\section{Background/Objectives}

Currently, accurate clinical models that predict short-term mortality in older ( $\geq 65$ years) emergency department (ED) patients are lacking. We aimed to develop and validate a prediction model for 30-day mortality in older ED patients that is easy to apply using variables that are readily available and reliably retrievable during the short phase of an ED stay.

\section{Methods}

Prospective multi-centre cohort study in older medical ED patients. The model was derived through logistic regression analyses, externally validated and compared with other well-known prediction models (Identification of Seniors at Risk (ISAR), ISARHospitalised Patients, Acute Physiology and Chronic Health Evaluation II (APACHE II) and Modified Early Warning Score (MEWS)).

\section{Results}

Within 30 days after presentation, $66(10.9 \%)$ of 603 patients in the derivation cohort and 105 (13.3\%) of 792 patients in the validation cohort died. The newly developed model included 6 predictors: age, $\geq 2$ abnormal vital signs, serum albumin, blood urea nitrogen, lactate dehydrogenase, and bilirubin. The discriminatory value of the model for mortality was very good with an AUC of 0.84 in the derivation and 0.83 in the validation cohort. The final model was excellently calibrated (Hosmer-Lemeshow pvalue 0.89 ). The discriminatory value of the model was significantly higher than that of the four risk stratification scores (highest AUC of 0.69 for ISAR score, p-value 0.007).

\section{Conclusion}

We developed and externally validated an accurate and simplified prediction model for 30-day mortality in older ED patients. This model may be useful to identify patients at risk of short-term mortality and to apply personalised medical care. 


\section{Introduction}

Early identification of high risk patients who visit an emergency department (ED) is important to optimise the diagnostic approach, care and outcome. In particular, older patients are at high risk of mortality after an ED visit. ${ }^{1}$ During ED care, important decisions regarding diagnostics and/or treatment intensity have to be made within a short timeframe. Especially for older patients, these decisions are strongly influenced by their mortality risk. ${ }^{2,3}$

Several triage and risk stratification scores ${ }^{4-6}$, as well as prediction models ${ }^{7,8}$ have been developed to identify those at high risk of dying but most of these tools have suboptimal discriminatory power with AUCs ranging from 0.56 to $0.77 .^{7,9-12}$ In addition, they often require information that is difficult to retrieve during the acute phase of an ED visit (e.g. premorbid cognitive functioning or comorbidity) ${ }^{4,6,7}$, or use mortality on the mid-long term (within 3-6 months) as outcome rather than mortality within the first 30 day. ${ }^{7,12,13}$ Finally, these scores and models have not been validated in older patients. ${ }^{6,8}$

We hypothesised that it is possible to construct a prediction model for older ED patients using items that are readily available in the ED. Additionally, we hypothesised that this model would be comparable or even better in predicting short-term mortality than four other well-known risk stratification scores (the Identification of Seniors at Risk (ISAR) score ${ }^{4}$, the ISAR-Hospitalised Patients (ISAR-HP) score ${ }^{14}$, the Acute Physiology and Chronic Health Evaluation II (APACHE II) score $^{6}$, and the Modified Early Warning Score (MEWS)).

Therefore, the aim of the present prospective multicentre study was to develop and validate a simplified prediction model for 30-day mortality in older medical patients using items that are easily and reliably retrievable during ED stay. In addition, we aimed to assess how our model performed relative to four other well-known risk stratification scores. 


\section{Materials and methods}

\section{Study design}

This study is part of the Risk Stratification in the Emergency Department in Acutely III Older Patients (RISE UP) study. This is a prospective multicentre study, which aims to identify predictors of 30-day mortality and other adverse outcomes in older ED patients. ${ }^{15}$ This Dutch study was approved by the medical ethics committees of Zuyderland Medical Centre (MC) and Maastricht University Medical Centre+ (MUMC+) (NL55867.096.15) and registered on clinicaltrials.gov (NCT02946398). The Stiell criteria for development of clinical prediction rules in emergency medicine were used for development and validation of our prediction model. ${ }^{15,16}$ This study was conducted and reported in line with the Transparent Reporting of a multivariate prediction model for Individual Prognosis or Diagnosis (TRIPOD) guidelines. ${ }^{17}$

\section{Study population}

Patients were prospectively recruited at the EDs of Zuyderland $M C$, a secondary care centre with two EDs, and MUMC+, a combined secondary/tertiary care centre. The participating hospitals all provide secondary and tertiary care for 607,000 inhabitants in the region Zuid-Limburg, one of the regions in Europe with the highest aging population. ${ }^{18}$ In the Netherlands, most patients visit the ED after referral by a general practitioner (GP). Only a small number of ED patients are referred by emergency medical services or are self-referred.

The study population consisted of medical ED patients aged 65 years or older who were treated by an internist or gastroenterologist. Patients were included only once during the study period. We excluded patients who were admitted to another ward than internal medicine or gastroenterology in order to focus on patients with complex medical problems and comorbidity.

The derivation cohort consisted of patients who were included consecutively at the EDs of two hospitals (Zuyderland MC location Heerlen and MUMC+), and provided informed consent in the period from July 14, 2016 until February 9, 2017 (Figure 5.1). Patients could be included 24 hours a day and 7 days a week. A research physician was often available to help with the study inclusions from Mondays through Fridays between 8 AM and 18 PM. Patients who were unable to speak Dutch, German, or English were excluded from the derivation cohort.

The external validation cohort consisted of patients who were not already prospectively included during the period from July to August 2016 and consecutive 
patients who visited the ED in the period from February until April 2017 in Zuyderland MC location Heerlen and Sittard.

\section{Data collection}

From electronic medical records, we collected data on age, sex, living situation and triage category (using the Manchester Triage System (MTS)). ${ }^{19}$ In addition, the following 6 vital signs upon ED arrival were retrieved: heart rate (HR), mean arterial blood pressure (MAP), respiratory rate (RR), oxygen saturation, temperature and Glasgow Coma Scale (GCS). When the RR or GCS were missing, we used paCO2 and descriptions in the medial records to deduce these values, similar to other studies. ${ }^{20,21}$ The Alert Verbal Painful Unresponsiveness (AVPU) scale was derived from the GCS. ${ }^{22}$ Furthermore, we collected routine laboratory results: glucose, sodium, potassium, creatinine, blood urea nitrogen (BUN), chloride, bicarbonate, calcium, phosphate, bilirubin, alkaline phosphatase, gamma-glutamyltransferase (GGT), alanine aminotransferase (ALT), aspartate aminotransferase (AST), lactate dehydrogenase $(\mathrm{LDH})$, albumin, c-reactive protein (CRP), haemoglobin, leukocytes, thrombocytes, international normalised ratio (INR) and activated partial thromboplastin time (aPTT).

To phenotype our patients, we retrieved information regarding cognitive functioning, functional status and comorbidity according to the Charlson Comorbidty Index (CCI). ${ }^{23}$ Cognitive functioning was categorised in dementia, mild cognitive impairment, delirium or normal cognitive function and based on the diagnosis of a geriatrician and/or on medical records. Functional status was assessed using a questionnaire for the Katz Activities of Daily Living (ADL) index score ${ }^{24}$, which was filled in for all hospitalised patients. For patients of the external validation cohort, baseline characteristics and the items incorporated in the developed prediction model were retrieved.

For the patients of the derivation cohort who were admitted in Zuyderland MC, we completed items of four risk stratification scores: 2 geriatric scores (ISAR and ISAR-HP) and 2 general risk stratification scores (APACHE II and MEWS). The ISAR-HP score is restricted to hospitalised patients and for the APACHE II score, a blood gas analysis is needed, which was not available in all patients. Therefore, the 4 scores were determined in a subset of patients.

\section{Outcome}

All-cause mortality within 30 days after ED presentation was used as outcome variable to determine the discriminatory value of our model and the four risk stratification 
scores. Data on mortality were verified using medical records, which were connected to the municipal administration office, and in case of uncertainty, the general practitioner was contacted.

\section{Candidate predictors}

We considered variables to be candidate predictors if they fulfilled three criteria: 1 ) they could be objectively and reliably retrieved during an ED visit, 2) they were available in at least $90 \%$ of the participants and 3) they were considered available in different EDs and therefore deemed to be generalisable. In this way, we ensured that the model would be easy to implement in routine ED care.

Cognitive and physical function, and comorbidity were not selected as candidate predictors because they were deemed to be not reliably or objectively retrievable in the short phase of the ED stay. Triage category was considered not generalisable because several triage systems are used in other EDs.

All laboratory results were tested for collinearity using the Pearson's correlation coefficient. In case of a strong correlation between two tests, the one with the strongest association with mortality was selected as candidate predictor. Linearity was visually checked for all possible continuous predictors and if necessary, continuous predictors were log transformed or dichotomised, based on their relationship with the outcome. In case of dichotomisation, laboratory reference values were used as cut-off points. Laboratory results of bilirubin, ALT and CRP reported below the lower detection limit were replaced by a value of half this detection limit.

Vital signs were dichotomised as being normal or abnormal based on the systemic inflammatory response syndrome (SIRS) and SOFA criteria. ${ }^{25,26}$ The following cut-off values were used: $H R>90 \mathrm{bpm}$ (i.e. tachycardia), $M A P<70, R R>20$ per minute (i.e. tachypnea), and GCS $<15$. For the GCS a cut-off value of $<15$ was chosen since patients with qualitative disturbance of consciousness (e.g. disorientation in place, person or time) can only receive a maximum GCS of 14 and because a qualitative disturbance of consciousness is a predictor of mortality. ${ }^{9}$ For abnormal temperature, we used a cutoff value of $<36$ or $>37.3^{\circ} \mathrm{C}$, since a cut-off value $>37.3^{\circ} \mathrm{C}$ is more appropriate for identifying infection in acute ill older patients than a value $>38.0^{\circ} \mathrm{C}^{27}$ For oxygen saturation, we applied a cut-off value of $<95 \%$.

\section{Statistical analysis}

The sample size for the derivation cohort was calculated by using 5 events per candidate predictor $^{28}$, and assuming that 30 -day mortality would be at least $10 \%{ }^{12,29}$ Under these assumptions, inclusion of 600 patients would provide enough events. We 
chose a more liberal approach of 5 events per candidate predictor because otherwise we would probably have to remove potentially very important predictors from the set of candidate predictors, which could lead to a model that performs suboptimally. In addition, to test the model's performance in data collected independently of the derivation data and to test for possible overfitting, we decided to externally validate the model. For this external validation cohort, approximately 100 events would be needed ${ }^{30}$, and since a mortality of $12.5 \%$ was expected, we considered a sample size of 800 patients necessary.

Baseline characteristics were computed on the observed data before imputing missing data. For candidate predictors, missing values were imputed using stochastic regression imputation with predictive mean matching (Supplemental Table S5.1). We identified possible predictors using univariable logistic regression analyses, and Odds Ratios (OR) with $95 \%$ confidence intervals $(95 \% \mathrm{Cl})$ were reported. Predictors with a P-value of $<0.20$ in the univariable logistic regression analysis were subsequently entered in the multivariable analysis. Abnormal vital signs were clustered to reduce the number of candidate predictors. The optimal number of abnormal vital signs was selected based on the value with highest area under the Receiver Operating Characteristic (ROC) curve.

We derived the model through backward stepwise elimination using a P-value of 0.10 for removal and determined the discriminatory ability of our model by calculating the area under the ROC curve (AUC). Internal validation was performed using 1000 bootstrap samples. This provided a shrinkage factor, and regression coefficients were adjusted accordingly to counteract the effect of overfitting.

Calibration of the prediction model was assessed by visually inspecting the calibration plot. We externally validated the model by calculating predicted risks of mortality for patients in the validation cohort, using the formulae: $1 /\left(1+\exp ^{\text {-linear predictor }}\right)$, in which the linear predictor is the sum of all predictor variables multiplied by their regression coefficients. An AUC, a calibration plot and the Hosmer-Lemeshow goodness-of-fit test were calculated to quantify the external validation of the prediction model.

AUCs of the four well-known risk stratification scores were calculated and compared with the new-built prediction model using the method of DeLong. ${ }^{31}$

To investigate for possible selection bias, we compared baseline characteristics of included and non-included patients. Comparisons between included and non-included patients were made using unpaired-t-tests for continuous variables with Gaussian distribution, Mann-Whitney tests for continuous non-Gaussian data and Pearson's chisquared or Fisher exact test for categorical data. 
All data were analysed using IBM SPSS Statistics for Windows, Version 24.0, IBM Corp., Armonk, N.Y., USA and R version 3.3.3.

\section{Results}

\section{Study population and patient characteristics}

During the prospective study period, 2109 older patients were eligible for inclusion in the derivation cohort (Figure 5.1). Of these, 1274 (60.4\%) were not asked for participation, 224 (10.6\%) were not included because they refused or were unable to give informed consent and $8(0.4 \%)$ because of a language barrier. Particularly during periods of crowding, not all eligible participants could be included in the derivation cohort. This resulted in 603 patients who were prospectively included in the derivation cohort.

The validation cohort consisted of 793 patients and all possible candidates could be included. One patient was lost to follow up and therefore 792 patients were available for analysis in the validation cohort.
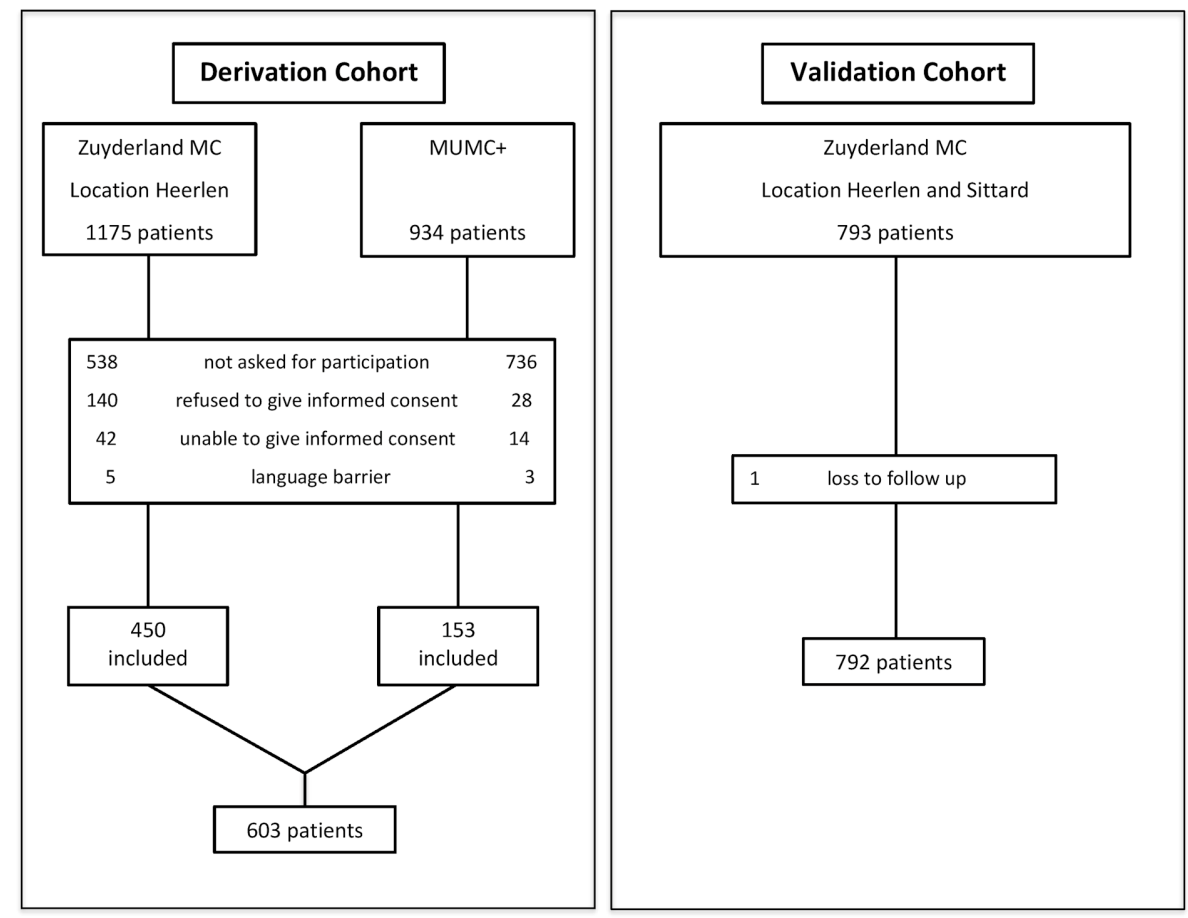

Figure 5.1 Overview of patient selection. 
The median age of patients was 79 years (IQR 73-85) in the derivation and 78 (IQR 72-85) years in the validation cohort (Table 5.1). Abnormal vital signs were highly prevalent in both cohorts but most laboratory values were within the reference range. Within 30 days, $66(10.9 \%)$ patients in the derivation cohort and 105 (13.3\%) patients in the validation cohort died.

\section{Selection of candidate predictors}

The following laboratory results were available in less than $90 \%$ of our patients: chloride, bicarbonate, phosphate, INR and aPTT. As BUN and creatinine ( $r=0.75)$, and ALT and AST ( $r=0.80)$ were highly correlated we decided to select BUN and AST as candidate predictors only because these two tests had the strongest association with mortality. Seven candidate predictors were not linearly associated with mortality, and were either dichotomised (sodium, potassium, bilirubin and leukocytes) or logarithmically transformed (alkaline phosphatase, GGT and AST). All 6 vital signs, if abnormal, were strongly associated with mortality, except abnormal temperature, which was eliminated as candidate predictor. The presence of $\geq 2$ of the 5 remaining vital signs yielded the best discriminatory value for mortality (AUC of $0.68,95 \%$ $\mathrm{Cl}=0.60-0.75)$, so having $\geq 2$ abnormal vital signs was selected as predictor.

\section{Univariable and multivariable logistic regression analysis}

The univariable logistic regression analysis identified 14 predictors of mortality ( $p$ value $<0.20$ ), which were used in the multivariable analysis (Table 5.2). Backward stepwise multivariable regression analysis resulted in a final model consisting of the following 6 predictors: age, $\geq 2$ abnormal vital signs, serum albumin, BUN, LDH and bilirubin $>20 \mu \mathrm{mol} / \mathrm{L}$. All items, except albumin, were positively associated with 30-day mortality. The AUC of the model in our derivation cohort was 0.84 ( $95 \% \mathrm{Cl}=0.78-0.89$ ). Internal validation provided a shrinkage factor of 0.94 and the AUC corrected for optimism was 0.82 (Table 5.3). Table 5.3 presents the model's regression coefficients before and after internal validation. The calibration plot (Figure 5.2) showed an excellent calibration between predicted and observed mortality. 
Table 5.1 Baseline characteristics of study cohorts ${ }^{a}$

\begin{tabular}{|c|c|c|c|}
\hline Characteristic & Reference values & $\begin{array}{c}\text { Derivation } \\
\text { cohort } \\
\mathrm{N}=603 \\
\end{array}$ & $\begin{array}{c}\text { Validation cohort }^{\mathrm{b}} \\
\mathrm{N}=792\end{array}$ \\
\hline Age, median (IQR), years & & $79(73-85)$ & $78(72-85)$ \\
\hline Male sex, $\mathrm{n} \%$ & & 311 (51.6) & $386(48.7)$ \\
\hline Living in nursing- or care home, $\mathrm{n} \%$ & & $51(8.5)$ & $66(8.3)$ \\
\hline Cognitive impairment or delirium, $\mathrm{n} \%$ & & $168(29.2)$ & NA \\
\hline Katz ADL index score, median (IQR) & & $0(0-2)$ & NA \\
\hline $\mathrm{CCl}$ score, median (IQR) & & $2(1-3)$ & NA \\
\hline \multicolumn{4}{|l|}{ Manchester triage category, $\mathrm{n} \%$} \\
\hline Red & & $3(0.5)$ & $6(0.8)$ \\
\hline Orange & & $66(11.1)$ & $145(18.4)$ \\
\hline Yellow & & $334(56.1)$ & $395(50.2)$ \\
\hline Green & & $189(31.8)$ & $234(29.7)$ \\
\hline Blue & & $3(0.5)$ & $7(0.9)$ \\
\hline \multicolumn{4}{|l|}{ Abnormal vital signs, $\mathrm{n} \%$} \\
\hline Tachycardia >90 bpm & & $211(35.5)$ & $283(36.0)$ \\
\hline $\mathrm{MAP}<70 \mathrm{mmHg}$ & & $41(7.0)$ & $66(8.5)$ \\
\hline Hypo- or hyperthermia $\left(<36\right.$ or $\left.>38^{\circ} \mathrm{C}\right)$ & & $154(26.5)$ & $217(28.0)$ \\
\hline Tachypnea $>20 / \mathrm{min}$ & & $137(22.7)$ & $161(20.3)$ \\
\hline O2-saturation $<95 \%$ & & $131(22.4)$ & $168(22.5)$ \\
\hline GCS $<15$ & & $91(15.1)$ & $128(16.2)$ \\
\hline$\geq 2$ abnormal vital signs ${ }^{c}$ & & $168(28.7)$ & $224(29.3)$ \\
\hline \multicolumn{4}{|l|}{ Laboratory results } \\
\hline Glucose, median (IQR), mmol/L & $<7.8$ & $7.1(6.0-8.8)$ & NA \\
\hline Sodium, mean (SD), $\mathrm{mmol} / \mathrm{L}$ & $135-145$ & $137(5.2)$ & $138(5.4)$ \\
\hline Potassium, mean (SD), $\mathrm{mmol} / \mathrm{L}$ & $3.5-5.0$ & $4.2(0.7)$ & $4.3(0.7)$ \\
\hline BUN, median (IQR), mmol/L & $3.5-7.5$ & $8.0(5.6-13.1)$ & $8.6(6.0-13.2)$ \\
\hline Creatinine, median (IQR), $\mu \mathrm{mol} / \mathrm{L}$ & $70-110$ & $97(75-137)$ & $100(78-138)$ \\
\hline Calcium, mean (SD), $\mathrm{mmol} / \mathrm{L}^{\mathrm{d}}$ & $2.20-2.60$ & $2.37(0.17)$ & $2.36(0.20)$ \\
\hline Bilirubin, median (IQR), $\mu \mathrm{mol} / \mathrm{L}$ & $<21$ & $10.0(6.0-15.0)$ & $9.0(6.0-15.0)$ \\
\hline Alkaline phosphatase, median (IQR), U/L & $q<98 \quad 3<115$ & $87(70-119)$ & $88(70-116)$ \\
\hline GGT, median (IQR), U/L & $+<38 \quad 3<55$ & $34(20-71)$ & $32(20-65)$ \\
\hline ALT, median (IQR), U/L & $q<34 \quad 3<45$ & $21(15-31)$ & $21(15-30)$ \\
\hline AST, median (IQR), U/L & $q<31 \quad \hat{0}<35$ & $27(20-43)$ & $28(21-43)$ \\
\hline LDH, median (IQR), U/L & $q<247 \quad \hat{o}<248$ & $226(186-295)$ & $232(190-307)$ \\
\hline Albumin, mean (SD), g/L & $35-50$ & $36.3(5.7)$ & $37.6(5.3)$ \\
\hline $\mathrm{CRP}$, median (IQR), mg/L & $<10$ & $31(7-88)$ & $27(6-77)$ \\
\hline Haemoglobin, mean (SD), mmol/L & q 7.5-10.0 § 8.5-11.0 & $7.4(1.6)$ & NA \\
\hline Leukocytes, median (IQR), x $10^{9} / \mathrm{L}$ & $4.0-10.0$ & $9.6(7.0-12.9)$ & NA \\
\hline Thrombocytes, mean (SD), $\times 10^{9} / \mathrm{L}$ & $150-400$ & $252(105)$ & NA \\
\hline \multicolumn{4}{|l|}{ Treatment, $\mathrm{n} \%$} \\
\hline Internal medicine & & $455(75.5)$ & $619(78.2)$ \\
\hline Admission, $\mathrm{n} \%$ & & 479 (79.4) & $644(81.3)$ \\
\hline 30-day mortality, n \% & & $66(10.9)$ & $105(13.3)$ \\
\hline
\end{tabular}

$A D L$, activities of daily living; ALT, alanine aminotransferase; AST, aspartate aminotransferase; BUN, blood urea nitrogen; CCI, Charlson Comorbidity Index; CRP, C-reactive protein; GCS, Glasgow Coma Scale; GGT, gammaglutamyltransferase; IQR, interquartile range; $L D H$, lactate dehydrogenase; MAP, mean arterial pressure; NA, not applicable; SD, standard deviation. To convert the laboratory tests values to conventional units multiply by the following conversion factors: for glucose to $\mathrm{mg} / \mathrm{dL}$ by 18.02 , sodium to $\mathrm{mEq} / \mathrm{L}$ by 1.0 , potassium to $\mathrm{mEq} / \mathrm{L}$ by $1.0, \mathrm{BUN}$ to $\mathrm{mg} / \mathrm{dL}$ by 2.8 , creatinine to $\mathrm{mg} / \mathrm{dL}$ by 0.0113 , calcium to $\mathrm{mg} / \mathrm{dL}$ by 4.0 , bilirubin to $\mathrm{mg} / \mathrm{dL}$ by 0.0585 , albumin to $\mathrm{g} / \mathrm{dL}$ by 0.1 , hemoglobin to $\mathrm{g} / \mathrm{dL}$ by 1.61 , leukocytes to $\times 10^{3} / \mu L$ by 1.0 and thrombocytes to $\times 10^{3} / \mu L$ by 1.0. ${ }^{\mathrm{a}}$ This table presents the observed data before imputation, all variables presented in this table were present in more than $90 \%$ of the participants; ${ }^{b}$ For the validation cohort, less items were retrieved; ${ }^{c} \geq 2$ of following abnormal vital signs: $\mathrm{HR}>90 \mathrm{bpm}, \mathrm{MAP}<70 \mathrm{mmHg}, \mathrm{RR}>20 / \mathrm{min}$, O2-saturation $<95 \%$ and $\mathrm{GCS}<15$; ${ }^{\mathrm{d}}$ Calcium was corrected for albumin by the formulae: calcium $+(0.02 *(40$-albumin $))$. 
Table 5.2 Univariable and multivariable logistic regression analysis for 30-day mortality.

\begin{tabular}{|c|c|c|c|c|}
\hline \multirow[t]{2}{*}{ Candidate predictor $^{a}$} & \multicolumn{2}{|c|}{ Univariable analysis } & \multicolumn{2}{|c|}{$\begin{array}{l}\text { Final model (multivariable } \\
\text { backwards elimination }{ }^{\text {b) }}\end{array}$} \\
\hline & $\begin{array}{l}\text { Odds Ratio } \\
(95 \% \mathrm{Cl})\end{array}$ & p-value & $\begin{array}{c}\text { Odds Ratio } \\
(95 \% \mathrm{Cl})\end{array}$ & p-value \\
\hline Age - per year increase & $1.07(1.03-1.11)$ & $<0.001$ & $1.05(1.01-1.10)$ & 0.014 \\
\hline Male & $1.07(0.64-1.78)$ & 0.802 & & \\
\hline Living in nursing- or care home & $3.22(1.62-6.43)$ & 0.001 & & \\
\hline$\geq 2$ abnormal vital signs ${ }^{c}$ & $4.57(2.70-7.76)$ & $<0.001$ & 3.27 (1.82-5.89) & $<0.001$ \\
\hline Glucose - per mmol/L increase & $0.99(0.92-1.06)$ & 0.662 & & \\
\hline Sodium $<135$ or $>145 \mathrm{mmol} / \mathrm{L}$ & $2.40(1.42-4.07)$ & 0.001 & & \\
\hline Potassium $<3.5$ or $>5.0 \mathrm{mmol} / \mathrm{L}$ & $2.69(1.56-4.66)$ & $<0.001$ & & \\
\hline BUN - per $5 \mathrm{mmol} / \mathrm{L}$ increase & $1.39(1.23-1.57)$ & $<0.001$ & $1.35(1.16-1.57)$ & $<0.001$ \\
\hline Calcium - per $0.1 \mathrm{mmol} / \mathrm{L}$ increase & $1.36(1.16-1.59)$ & $<0.001$ & & \\
\hline Bilirubin $>20 \mu \mathrm{mol} / \mathrm{L}$ & $3.33(1.87-5.94)$ & $<0.001$ & $2.54(1.26-5.12)$ & 0.009 \\
\hline Alkaline Phosphatase - per log U/L increase & $5.84(2.47-13.77)$ & $<0.001$ & & \\
\hline GGT - per log U/L increase & $2.10(1.25-3.51)$ & 0.005 & & \\
\hline AST - per log U/L increase & $3.07(1.54-6.11)$ & 0.001 & & \\
\hline LDH - per $100 \mathrm{U} / \mathrm{L}$ increase & $1.25(1.13-1.38)$ & $<0.001$ & $1.14(1.02-1.27)$ & 0.024 \\
\hline Albumin - per $\mathrm{g} / \mathrm{L}$ increase & $0.86(0.83-0.90)$ & $<0.001$ & $0.89(0.84-0.93)$ & $<0.001$ \\
\hline $\mathrm{CRP}$ - per $10 \mathrm{mg} / \mathrm{L}$ increase & $1.06(1.03-1.09)$ & $<0.001$ & & \\
\hline Haemoglobin - per mmol/L increase & $0.92(0.78-1.07)$ & 0.260 & & \\
\hline Leukocytes $>10.0 \times 10^{9} / \mathrm{L}$ & $1.31(0.78-2.18)$ & 0.307 & & \\
\hline Thrombocytes - per $10 \times 10^{9} / L$ increase & $1.01(0.99-1.03)$ & 0.468 & & \\
\hline
\end{tabular}

$A L T$, alanine aminotransferase; AST, aspartate aminotransferase; $\beta$, regression coefficient; BUN, blood urea nitrogen; $\mathrm{Cl}$, confidence interval; CRP, C-reactive protein; GGT, gamma-glutamyltransferase; LDH, lactate dehydrogenase; Log, logarithm. ${ }^{a}$ Missing values of candidate predictors were imputed using stochastic regression imputation with predictive mean matching (Supplementary Table S5.2); ${ }^{\mathrm{b}}$ All predictors with $\mathrm{p}$-value $<0.20$ were included in the multivariable analysis; ${ }^{c} \geq 2$ of following abnormal vital signs: $\mathrm{HR}>90 \mathrm{bpm}$, $\mathrm{MAP}<70 \mathrm{mmHg}, \mathrm{RR}>20 / \mathrm{min}, \mathrm{O}_{2}$-saturation $<95 \%$ or $\mathrm{GCS}<15$

Table 5.3 Adjusted coefficients and discriminatory ability of the final model after internal and external validation.

\begin{tabular}{|c|c|c|c|c|}
\hline \multirow[t]{2}{*}{ Predictor } & \multicolumn{2}{|c|}{ Derivation cohort } & \multirow{2}{*}{$\begin{array}{l}\text { Internal validation } \\
\text { shrunk coefficient }^{\mathrm{a}}\end{array}$} & \multirow[t]{2}{*}{ Validation cohort } \\
\hline & $\beta$ & p-value & & \\
\hline Age - per year increase & 0.053 & 0.014 & 0.050 & \\
\hline$\geq 2$ abnormal vital signs ${ }^{b}$ & 1.186 & $<0.001$ & 1.115 & \\
\hline Albumin - per g/L increase & -0.120 & $<0.001$ & -0.112 & \\
\hline BUN - per $5 \mathrm{mmol} / \mathrm{L}$ increase & 0.302 & $<0.001$ & 0.284 & \\
\hline LDH - per $100 \mathrm{U} / \mathrm{L}$ increase & 0.128 & 0.024 & 0.120 & \\
\hline Bilirubin $>20 \mu \mathrm{mol} / \mathrm{L}$ & 0.931 & 0.009 & 0.875 & \\
\hline Intercept & -4.053 & & -3.908 & \\
\hline Model & \multicolumn{2}{|c|}{ AUC $(95 \% \mathrm{Cl})$} & AUC & AUC (95\% Cl) \\
\hline Final Model $^{c}$ & \multicolumn{2}{|c|}{$0.84(0.78-0.89)$} & 0.82 & $0.83(0.78-0.87)$ \\
\hline
\end{tabular}

$A U C$, area under de curve; $\beta$, regression coefficient; BUN, blood urea nitrogen; $\mathrm{Cl}$, confidence interval; LDH, lactate dehydrogenase. ${ }^{a}$ Coefficients shrunk by multiplying with the shrinkage factor (0.94). The intercept was subsequently re-estimated; ${ }^{b} \geq 2$ of following abnormal vital signs: $\mathrm{HR}>90 \mathrm{bpm}, \mathrm{MAP}<70 \mathrm{mmHg}, \mathrm{RR}$ $>20 /$ min, O2-saturation $<95 \%$ and GCS $<15$; ${ }^{C}$ Formulae of the final model: $P(30$-day mortality $)=1 /(1+\exp (-(-$ $3.908+0.050 *$ age $+1.115^{*} \geq 2$ abnormal vital signs $-0.112^{*}$ albumin $+0.284 *(B U N / 5)+0.120 *(L D H / 100)+$ $0.875 *$ bilirubin $>20)$ )). 


\section{External validation}

The final model (i.e. RISE UP score, Table 5.3 legend) was used to calculate the predicted 30-day mortality risk in the 792 patients of the validation cohort. The AUC of our final model was $0.83(95 \% \mathrm{Cl}=0.78-0.87)$ and the calibration plot showed excellent calibration as the plot followed the 45-degree line of perfect calibration. This was confirmed by a non-significant Hosmer-Lemeshow test with a p-value of 0.89 .

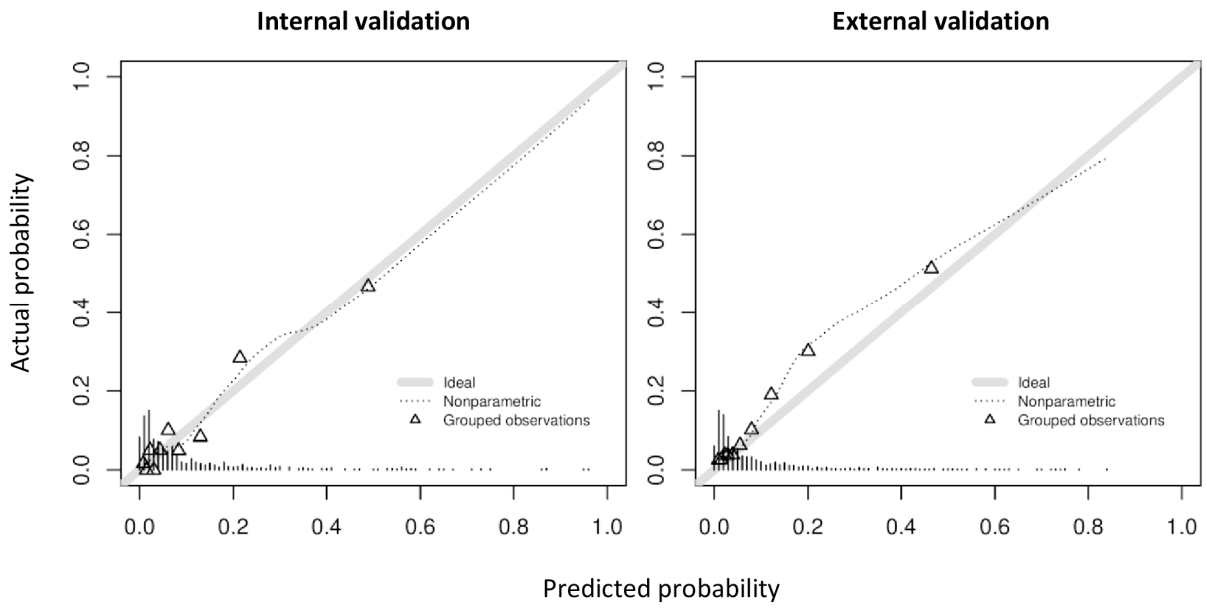

Figure 5.2 Calibration plots after internal and external validation. Calibration plot of the predicted 30-day mortality ( $x$ axis) versus the observed 30-day mortality ( $y$ axis) is represented by the dotted line. Calibration of the model after internal validation is represented by the left calibration plot and after external validation by the right calibration plot. Predicted risks were derived from our final model (Table 5.3 legend). The solid diagonal line represents perfect calibration. Both plots follow the 45-degree line of perfect calibration, which indicates excellent calibration.

\section{Comparison with the four other risk stratification scores}

The ISAR, ISAR-HP, APACHE II and MEWS scores were calculated in the subset of 366 patients who were admitted to the hospital. The AUC of the scores were ranging from 0.57 for the MEWS score to 0.69 for the ISAR and ISAR-HP score (Table 5.4). The AUC of the newly developed RISE UP score in this subset of patients was $0.83(95 \%$ $\mathrm{Cl}=0.77-0.90)$ and this was significantly higher than that of the four other scores (all p-values <0.05). 
Table 5.4 Comparison of the AUCs of risk stratification scores and the RISE UP score in a subset of admitted patients $(n=366)$.

\begin{tabular}{lcc}
\hline Risk stratification score & AUC (95\% Cl) & $\begin{array}{c}\text { Method of deLong }^{\text {a }} \\
\text { (p-value) }\end{array}$ \\
\hline ISAR score & $0.69(0.60-0.77)$ & 0.007 \\
ISAR-HP score & $0.67(0.59-0.75)$ & 0.001 \\
APACHE II score & $0.66(0.58-0.75)$ & 0.001 \\
MEWS & $0.57(0.47-0.66)$ & $<0.001$ \\
RISE UP score & $0.83(0.77-0.90)$ & $\mathrm{NA}$ \\
\hline
\end{tabular}

APACHE II, Acute Physiology and Chronic Health Evaluation II; AUC, area under the curve; $\mathrm{Cl}$, confidence interval; ISAR, Identification of Seniors at Risk; ISAR-HP, ISAR-Hospitalised Patients; MEWS, Modified Early Warning Score, RISE UP: Risk Stratification in the Emergency Department in Acutely III Older Patients. ${ }^{a}$ Comparison of the AUC of the risk stratification score with the AUC of the RISE UP score (method of delong).

\section{Selection of patients}

When we compared the baseline characteristics of included and non-included patients, we found no significant differences in baseline characteristics and outcomes, except that more patients in the non-included patient group were triaged more urgently $(p=0.003)$, the MAP was more frequently $<70 \mathrm{mmHg}(p=0.02)$ and serum LDH was slightly lower ( $p=0.03$ ) (Supplemental Table S5.2).

\section{Discussion}

In this prospective, multicentre study, we developed and externally validated a prediction model that can be used to predict 30-day all-cause mortality in older medical ED patients using easily retrievable items. The model performed very well in the validation population with an AUC of $0.83(95 \% \mathrm{Cl}=0.78-0.87)$ and calibration was excellent. The discriminatory ability of the model was significantly better than that of four other well-known risk stratification scores, with the highest AUC of 0.69 for the ISAR score. Using the RISE UP score, it is possible to identify those older medical ED patients at high and at low risk of dying within 30 days.

The RISE UP score consists of six easily and reliably retrievable variables: age, $\geq 2$ abnormal vital signs, albumin, BUN, LDH and bilirubin. These predictors provide face validity of the prediction model, because they are indicators of underlying disease. Abnormal vital signs are associated with mortality and used in several risk stratification scores ${ }^{5,6}$, but a combination of two or more abnormal vital signs is not often incorporated in prediction models. However, one ICU study showed that a 
combination of more than one abnormal vital signs predicts mortality better than separate vital signs. ${ }^{32}$ Age and the four laboratory tests in our model were identified as predictors of mortality in other studies as well. ${ }^{7,8,12,33-35}$ Albumin, BUN, LDH and bilirubin are markers of severe diseases, such as sepsis, malignancy, renal failure, upper gastrointestinal bleeding, ischemia or shock, haemolysis and liver- and/or bile duct disease. Since these severe diseases and conditions are often present in medical older ED patients and related to mortality it is no surprise that these laboratory tests contribute largely to our model.

Our prediction model has a good discriminatory ability and is easy to implement in emergency care. Compared to other risk stratification scores and prediction models, the discriminatory ability of the RISE UP score is better. The ISAR-score has found to have an AUC of 0.80 for 30 -day mortality in a recent meta-analysis. ${ }^{36}$ We found a lower AUC, which may reflect the difficulty in retrieving the necessary information (e.g. premorbid functioning) in the ED phase. The ISAR-HP score was developed to predict functional decline within 90-days in older patients admitted to the department of internal medicine and was recently found to predict functional decline and 90-day mortality (AUC of 0.69) in a Dutch study. ${ }^{37}$ Although we used the ISAR-HP to predict mortality only, the AUC of 0.67 we found is comparable to the one found in this Dutch study. Similarly to the ISAR score, the ISAR-HP includes premorbid physical functioning, which is, in our opinion, less reliably retrievable during ED stay. The APACHE II score is used to predict mortality in ICU patients and assigns points to 12 physiological variables, age and the previous health status of the patient. Its discriminatory value for in-hospital mortality is however moderate to very good with AUCs ranging from $0.62-0.88 .{ }^{38}$ In one Chinese study, the AUC of the APACHE II score in older ICU patients was 0.76, which is higher than the AUC we found (AUC of 0.66). ${ }^{39}$ However, most of the patients we included were non-critically ill patients, which may explain the difference. In addition, the APACHE II score is less feasible in the ED due to the use of arterial blood gas, which is not routinely measured. To date, the APACHE II score has not yet been validated in the older ED population. The MEWS has been shown to predict mortality in older ED patients with varying discriminatory values (AUCs ranging from 0.61-0.89). ${ }^{40}$ In one Japanese study ${ }^{41}$, the AUC for predicting inhospital mortality in older ED patients was 0.72 , which is higher than what we found (AUC of 0.57). This difference can be explained because in this Japanese study only ambulance patients were included. In contrast, a Dutch study showed an AUC of 0.56 for the MEWS in older ED patients ( $\geq 70$ years) with sepsis, which is comparable with what we found. ${ }^{11}$ Another recently developed Dutch prediction model for 90-day mortality in general older ( $\geq 70$ years) ED patients (APOP screener) yields an AUC of 
0.67 and contains items such as cognitive impairment. ${ }^{7}$ The lower AUCs and their less reliable and/or difficult retrievable items make these other scores, in our opinion, less useful in older ED patients.

The model we developed can be an important tool to predict short-term mortality in older ED patients, despite the fact that age is the only obvious aging characteristic. Although we excluded some geriatric variables, we still developed a model with high discriminatory ability for short-term mortality. It is intriguing that the items we included, measured at an early stage of presentation in the ED, are such strong predictors of 30-day mortality. The variables representing disease severity (vital signs and laboratory tests) were selected in the logistic regression analysis over living in nursing- and care home. This yields a new hypothesis that the severity of disease is highly important in predicting short-term mortality and is possibly more important than the premorbid state. Since our model is easy to use, not time-consuming to fill in and has the best discriminatory ability for short-term we recommend to determine the laboratory tests incorporated in our model routinely in older medical emergency department patients.

\section{Strengths and limitations}

Our study has several limitations. First, it was developed in medical (i.e. internal medicine and gastroenterology) ED patients and may therefore not be applicable in older patients who visit the ED for other non-medical specialties. We included these patients as these are in general patients with complex medical problems and comorbidity. Second, especially in non-medical patients, the laboratory tests incorporated in the RISE UP score will probably not all be routinely measured. It is possible that extra laboratory tests are needed in these patients in order to fill in our score. However, if future studies show a good discriminatory value of our model in these patients, these tests could easily be ordered with only minor additional costs.

Third, the results in our derivation cohort may have been biased due to selective inclusion of patients. However, we investigated possible selection bias by comparing the baseline characteristics and outcomes of the included with non-included patients and found no evidence for this. In addition, the model was tested in a validation cohort without finding an important influence on the discriminatory value of the model.

The strengths of the study are its prospective multicentre design and inclusion of older patients with diverse medical problems. Our study population is representative 
for the aging ED population worldwide since our study was conducted in one of the regions in Europe with the highest aging population. The RISE UP score, developed according to the Stiell criteria, has the advantage of identifying both patients with high and with low risks of mortality, as shown by the calibration plot. Therefore, our model may be used to personalise diagnostics, treatment and care decisions for older ED patients in order to improve their outcome and/or well-being.

\section{Clinical implications and future research}

Our model is planned to be implemented in routine practice by development of an online calculator and/or implementation in an electronic medical record system. With such a tool, the predicted probability of mortality for the individual patient can be easily generated within the first 2 hours of the ED visit. The estimation of prognosis by the RISE UP score should be used together with information regarding the patient's characteristics, medical problem and wishes in order to guide a more patient-centred care. For instance, this could result in the decision not to perform invasive diagnostical or treatment procedures, or, by intensifying care in high risk patients. For that reason, it will be very important to determine whether its prediction will improve patient care and outcomes in future prospective randomised impact studies. In addition, to assess the generalisability of our model in different settings, future research should focus on externally validating our model in older patients in other EDs and in patients presenting for other specialties.

\section{Conclusions}

In conclusion, we developed and externally validated an accurate and easily applicable prediction model for 30-day mortality in older ED patients. This model contributes to a faster recognition of patients at risk of short-term mortality and may contribute to application of more personalised treatments.

\section{Acknowledgements}

We are grateful for the help from geriatricians E. Pijpers and K. Hurkens in the study development. In addition, we thank the medical and nursing staff of the emergency departments of Zuyderland Medical Centre and Maastricht University Medical Centre+ for their contribution to our study and most of all, all patients who participated in the study. 


\section{References}

1. Aminzadeh F, Dalziel WB. Older adults in the emergency department: a systematic review of patterns of use, adverse outcomes, and effectiveness of interventions. Ann Emerg Med. 2002;39:238-47.

2. Gill TM. The Central Role of Prognosis in Clinical Decision Making. Jama. 2012;307:199-200.

3. Yourman LC, Lee SJ, Schonberg MA, Widera EW, Smith AK. Prognostic indices for older adults: a systematic review. JAMA. 2012;307:182-92.

4. McCusker J, Bellavance F, Cardin S, Trepanier S, Verdon J, Ardman O. Detection of older people at increased risk of adverse health outcomes after an emergency visit: the ISAR screening tool. J Am Geriatr Soc. 1999;47:1229-37.

5. Subbe CP, Kruger M, Rutherford P, Gemmel L. Validation of a modified Early Warning Score in medical admissions. QJM. 2001;94:521-6.

6. Knaus WA, Draper EA, Wagner DP, Zimmerman JE. APACHE II: a severity of disease classification system. Crit Care Med. 1985;13:818-29.

7. de Gelder J, Lucke JA, de Groot B, Fogteloo AJ, Anten S, Mesri K, et al. Predicting adverse health outcomes in older emergency department patients: the APOP study. Neth J Med. 2016;74:342-52.

8. Ha DT, Dang TQ, Tran NV, Pham TN, Nguyen ND, Nguyen TV. Development and validation of a prognostic model for predicting 30-day mortality risk in medical patients in emergency department (ED). Sci Rep. 2017;7:46474.

9. Carpenter CR, Shelton E, Fowler S, Suffoletto B, Platts-Mills TF, Rothman RE, et al. Risk factors and screening instruments to predict adverse outcomes for undifferentiated older emergency department patients: a systematic review and meta-analysis. Acad Emerg Med. 2015;22:1-21.

10. Steiner D, Renetseder F, Kutz A, Haubitz S, Faessler L, Anderson JB, et al. Performance of the Manchester Triage System in Adult Medical Emergency Patients: A Prospective Cohort Study. J Emerg Med. 2016;50:678-89.

11. de Groot B, Stolwijk F, Warmerdam M, Lucke JA, Singh GK, Abbas M, et al. The most commonly used disease severity scores are inappropriate for risk stratification of older emergency department sepsis patients: an observational multi-centre study. Scand J Trauma Resusc Emerg Med. 2017;25:91.

12. Buurman BM, van Munster BC, Korevaar JC, Abu-Hanna A, Levi M, de Rooij SE. Prognostication in acutely admitted older patients by nurses and physicians. J Gen Intern Med. 2008;23:1883-9.

13. Smolin B, Levy Y, Sabbach-Cohen E, Levi L, Mashiach T. Predicting mortality of elderly patients acutely admitted to the Department of Internal Medicine. Int J Clin Pract. 2015;69:501-8.

14. Hoogerduijn JG, Buurman BM, Korevaar JC, Grobbee DE, de Rooij SE, Schuurmans MJ. The prediction of functional decline in older hospitalised patients. Age Ageing. 2012;41:381-7.

15. Zelis N, Buijs J, de Leeuw PW, van Kuijk SMJ, Stassen PM. Study protocol for a multicentre prospective cohort study to identify predictors of adverse outcome in older medical emergency department patients (the Risk Stratification in the Emergency Department in Acutely III Older Patients (RISE UP) study). BMC Geriatr. 2019;19:65.

16. Stiell IG, Wells GA. Methodologic standards for the development of clinical decision rules in emergency medicine. Ann Emerg Med. 1999;33:437-47.

17. Collins GS, Reitsma JB, Altman DG, Moons KG. Transparent reporting of a multivariable prediction model for individual prognosis or diagnosis (TRIPOD): the TRIPOD statement. BMJ (Clinical research ed). 2015;350:g7594.

18. Peter Ekamper NvN. Vergrijzing. Demos2018. p. 5-7.

19. Mackway-Jones K, Marsden J, Windle J, Manchester Triage G. Emergency triage. 2nd ed. ed. Malden, Mass. Blackwell Pub.; 2006.

20. Hilderink MJ, Roest AA, Hermans M, Keulemans YC, Stehouwer CD, Stassen PM. Predictive accuracy and feasibility of risk stratification scores for 28-day mortality of patients with sepsis in an emergency department. Eur J Emerg Med. 2015;22:331-7.

21. Howell MD, Donnino MW, Talmor D, Clardy P, Ngo L, Shapiro NI. Performance of severity of illness scoring systems in emergency department patients with infection. Acad Emerg Med. 2007;14:709-14. 
22. Salottolo K, Carrick M, Johnson J, Gamber M, Bar-Or D. A retrospective cohort study of the utility of the modified early warning score for interfacility transfer of patients with traumatic injury. BMJ Open. 2017;7:e016143.

23. Charlson ME, Pompei $\mathrm{P}$, Ales KL, MacKenzie CR. A new method of classifying prognostic comorbidity in longitudinal studies: development and validation. J Chronic Dis. 1987;40:373-83.

24. Katz S, Ford AB, Moskowitz RW, Jackson BA, Jaffe MW. Studies of illness in the aged. The Index of ADL: a standardized measure of biological and psychosocial function. JAMA. 1963;185:914-9.

25. Bone RC, Balk RA, Cerra FB, Dellinger RP, Fein AM, Knaus WA, et al. Definitions for sepsis and organ failure and guidelines for the use of innovative therapies in sepsis. The ACCP/SCCM Consensus Conference Committee. American College of Chest Physicians/Society of Critical Care Medicine. Chest. 1992;101:1644-55.

26. Vincent JL, Moreno R, Takala J, Willatts S, De Mendonca A, Bruining H, et al. The SOFA (Sepsis-related Organ Failure Assessment) score to describe organ dysfunction/failure. On behalf of the Working Group on Sepsis-Related Problems of the European Society of Intensive Care Medicine. Intensive Care Med. 1996;22:707-10.

27. Singler K, Bertsch T, Heppner HJ, Kob R, Hammer K, Biber R, et al. Diagnostic accuracy of three different methods of temperature measurement in acutely ill geriatric patients. Age Ageing. 2013;42:740-6.

28. Vittinghoff $E$, McCulloch CE. Relaxing the rule of ten events per variable in logistic and Cox regression. Am J Epidemiol. 2007;165:710-8.

29. Magdelijns FJ, Schepers L, Pijpers E, Stehouwer CD, Stassen PM. Unplanned readmissions in younger and older adult patients: the role of healthcare-related adverse events. Eur J Med Res. 2016;21:35.

30. Collins GS, Ogundimu EO, Altman DG. Sample size considerations for the external validation of a multivariable prognostic model: a resampling study. Stat Med. 2016;35:214-26.

31. DeLong ER, DeLong DM, Clarke-Pearson DL. Comparing the areas under two or more correlated receiver operating characteristic curves: a nonparametric approach. Biometrics. 1988;44:837-45.

32. Barfod C, Lauritzen MM, Danker JK, Soletormos G, Forberg JL, Berlac PA, et al. Abnormal vital signs are strong predictors for intensive care unit admission and in-hospital mortality in adults triaged in the emergency department - a prospective cohort study. Scand J Trauma Resusc Emerg Med. 2012;20:28.

33. Jellinge ME, Henriksen DP, Hallas P, Brabrand M. Hypoalbuminemia is a strong predictor of 30-day allcause mortality in acutely admitted medical patients: a prospective, observational, cohort study. PloS One. 2014;9:e105983.

34. de Gelder J, Lucke JA, Heim N, de Craen AJ, Lourens SD, Steyerberg EW, et al. Predicting mortality in acutely hospitalized older patients: a retrospective cohort study. Intern Emerg Med. 2016;11:587-94.

35. Loekito E, Bailey J, Bellomo R, Hart GK, Hegarty C, Davey P, et al. Common laboratory tests predict imminent death in ward patients. Resuscitation. 2013;84:280-5.

36. Galvin R, Gilleit $Y$, Wallace E, Cousins G, Bolmer M, Rainer T, et al. Adverse outcomes in older adults attending emergency departments: a systematic review and meta-analysis of the Identification of Seniors At Risk (ISAR) screening tool. Age Ageing. 2017;46:179-86.

37. de Gelder J, Haenen E, Lucke JA, Klop HG, Blomaard LC, Smit RAJ, et al. Optimising the ISAR-HP to screen efficiently for functional decline in older patients. Neth J Med. 2017;75:379-85.

38. Minne L, Abu-Hanna A, de Jonge E. Evaluation of SOFA-based models for predicting mortality in the ICU: A systematic review. Crit Care (London, England). 2008;12:R161-R.

39. Qiao $Q$, Lu G, Li M, Shen Y, Xu D. Prediction of outcome in critically ill elderly patients using APACHE II and SOFA scores. J Int Med Res. 2012;40:1114-21.

40. Nannan Panday RS, Minderhoud TC, Alam N, Nanayakkara PWB. Prognostic value of early warning scores in the emergency department (ED) and acute medical unit (AMU): A narrative review. Eur J Intern Med. 2017;45:20-31.

41. Mitsunaga T, Hasegawa I, Uzura M, Okuno K, Otani K, Ohtaki Y, et al. Comparison of the National Early Warning Score (NEWS) and the Modified Early Warning Score (MEWS) for predicting admission and in-hospital mortality in elderly patients in the pre-hospital setting and in the emergency department. PeerJ. 2019;7:e6947. 


\section{Supplemental data}

Table S5.1 Overview imputed candidate predictors.

\begin{tabular}{|c|c|c|}
\hline Imputed candidate predictors & $\begin{array}{c}\text { Derivation cohort } \\
(n=603) \\
n(\%)\end{array}$ & $\begin{array}{l}\text { Validation cohort } \\
\text { (n=792) } \\
n(\%)\end{array}$ \\
\hline Heart rate & $9(1.5)$ & $5(0.6)$ \\
\hline MAP & $19(3.2)$ & $20(2.5)$ \\
\hline Temperature & $22(3.6)$ & NA \\
\hline Oxygen saturation & $19(3.2)$ & $45(5.7)$ \\
\hline Glucose & $21(3.5)$ & NA \\
\hline Sodium & $12(2.0)$ & NA \\
\hline Potassium & $12(2.0)$ & NA \\
\hline BUN & $21(3.5)$ & $28(3.5)$ \\
\hline Calcium & $51(8.5)$ & NA \\
\hline Bilirubin & $36(6.0)$ & $65(8.2)$ \\
\hline Alkaline Phosphatase & $32(5.3)$ & NA \\
\hline GGT & $25(4.1)$ & NA \\
\hline ALT & $21(3.5)$ & NA \\
\hline AST & $30(5.0)$ & NA \\
\hline $\mathrm{LDH}$ & $32(5.3)$ & $32(4.0)$ \\
\hline Albumin & $53(8.8)$ & $70(8.8)$ \\
\hline CRP & $18(3.0)$ & NA \\
\hline Haemoglobin & $8(1.3)$ & NA \\
\hline Leukocytes & $13(2.2)$ & NA \\
\hline Thrombocytes & $20(3.3)$ & NA \\
\hline
\end{tabular}

$A L T$, alanine aminotransferase; AST, aspartate aminotransferase; BUN, blood urea nitrogen; CRP, C-reactive protein; GGT, gamma-glutamyltransferase; LDH, lactate dehydrogenase; MAP, mean arterial pressure; NA, not applicable. 
Table S5.2 Baseline characteristics of the included and non-included patients.

\begin{tabular}{|c|c|c|c|}
\hline Characteristic & Reference values & $\begin{array}{l}\text { Included }^{\mathrm{a}} \\
(\mathrm{n}=603)\end{array}$ & $\begin{array}{c}\text { Non-included }^{\mathrm{a}} \\
(\mathrm{n}=\mathbf{2 0 0})\end{array}$ \\
\hline Median age (IQR), years & & $79(73-85)$ & $78(72-85)$ \\
\hline Male sex, $\mathrm{n}(\%)$ & & $311(51.6)$ & $100(50.0)$ \\
\hline Living in nursing- or care home, $\mathrm{n}(\%)$ & & $51(8.5)$ & $23(11.7)$ \\
\hline \multicolumn{4}{|l|}{ Manchester triage category, $\mathrm{n}(\%)$} \\
\hline Orange & & $66(11.1)^{d}$ & $38(19.2)^{d}$ \\
\hline Yellow & & $334(56.1)$ & $108(54.5)$ \\
\hline Green & & $189(31.8)$ & $51(25.8)$ \\
\hline \multicolumn{4}{|l|}{ Abnormal vital signs, $\mathrm{n}(\%)$} \\
\hline Tachycardia >90 bpm & & $211(35.5)$ & $68(34.2)$ \\
\hline MAP $<70 \mathrm{mmHg}$ & & $41(7.0)^{d}$ & $24(12.5)^{d}$ \\
\hline Hypo- or hyperthermia $\left(<36\right.$ or $\left.>38^{\circ} \mathrm{C}\right)$ & & $154(26.5)$ & $58(29.6)$ \\
\hline Tachypnea >20/min & & $137(22.7)$ & $44(22.0)$ \\
\hline O2-saturation $<95 \%$ & & $131(22.4)$ & $45(23.2)$ \\
\hline GCS $<15$ & & $91(15.1)$ & $38(19.0)$ \\
\hline$\geq 2$ abnormal vital signs $^{\mathrm{b}}$ & & $168(28.7)$ & $63(32.1)$ \\
\hline \multicolumn{4}{|l|}{ Laboratory results } \\
\hline Mean sodium (SD) , mmol/L & $135-145$ & $137(5.2)$ & $138(6.4)$ \\
\hline Mean potassium (SD), $\mathrm{mmol} / \mathrm{L}$ & $3.5-5.0$ & $4.2(0.7)$ & $4.3(0.7)$ \\
\hline Median BUN (IQR), mmol/L & $3.5-7.5$ & $8.0(5.6-13.1)$ & $9.0(6.1-13.6)$ \\
\hline Median creatinine (IQR), $\mu \mathrm{mol} / \mathrm{L}$ & 70-110 & 97 (75-137) & $103(82-145)$ \\
\hline Mean calcium (SD), $\mathrm{mmol} / \mathrm{L}^{\mathrm{c}}$ & $2.20-2.60$ & $2.37(0.17)$ & $2.40(0.23)$ \\
\hline Median bilirubin (IQR), $\mu \mathrm{mol} / \mathrm{L}$ & $<21$ & $10.0(6.0-15.0)$ & $9.0(5.0-15.0)$ \\
\hline Median alkaline phosphatase (IQR), U/L & $q<98 \quad \hat{o}<115$ & 87 (70-119) & $83(65-110)$ \\
\hline Median GGT (IQR), U/L & $q<38 \quad \hat{o}<55$ & $34(20-71)$ & $29(21-66)$ \\
\hline Median ALT (IQR), U/L & $9<34 \quad \hat{o}<45$ & $21(15-31)$ & $22(16-30)$ \\
\hline Median AST (IQR), U/L & Q $<31$ ふर<35 & $27(20-43)$ & $26(20-43)$ \\
\hline Median LDH (IQR), U/L & $q<247 \quad \hat{o}<248$ & $226(186-295)^{d}$ & $216(175-277)^{d}$ \\
\hline Mean albumin (SD), g/L & $35-50$ & $36.3(5.7)$ & $37.0(5.0)$ \\
\hline Median CRP (IQR), mg/L & $<10$ & $31(7-88)$ & $31(4-80)$ \\
\hline \multicolumn{4}{|l|}{ Treatment, n (\%) } \\
\hline Internal medicine & & $455(75.5)$ & $153(76.5)$ \\
\hline Admission & & $479(79.4)$ & $171(85.5)$ \\
\hline 30-day mortality, n (\%) & & 66 (10.9) & $30(15.0)$ \\
\hline
\end{tabular}

ALT, alanine aminotransferase; AST, aspartate aminotransferase; BUN, blood urea nitrogen; CRP, C-reactive protein; GCS, Glasgow Coma Scale; GGT, gamma-glutamyltransferase; IQR, interquartile range; LDH, lactate dehydrogenase; MAP, mean arterial pressure; NA, not applicable; SD, standard deviation. To convert the laboratory tests values from SI-units to conventional units multiply by the following conversion factors: for sodium to $\mathrm{mEq} / \mathrm{L}$ by 1.0 , potassium to $\mathrm{mEq} / \mathrm{L}$ by 1.0 , BUN to $\mathrm{mg} / \mathrm{dL}$ by 2.8 , creatinine to $\mathrm{mg} / \mathrm{dL}$ by 0.0113 , calcium to $\mathrm{mg} / \mathrm{dL}$ by 4 , bilirubin to $\mathrm{mg} / \mathrm{dL}$ by 0.0585 and albumin to $\mathrm{g} / \mathrm{dL}$ by 0.1 . ${ }^{\mathrm{a}}$ In total, 603 patients were included in the derivation cohort during the prospective study period (included patients). The non-included patients represent a sample of 200 patients who were not included during the prospective study period for reasons other than refusal; ${ }^{b} \geq 2$ of following abnormal vital signs: HR $>90$ bpm, $\mathrm{MAP}<70 \mathrm{mmHg}, \mathrm{RR}>20 / \mathrm{min}, \mathrm{O} 2$-saturation $<95 \%$ and $\mathrm{GCS}<15$; ' Calcium was corrected for albumin by the formula: calcium $+(0.02 *(40$-albumin $)) ;{ }^{\mathrm{d}}$ There were no significant differences in baseline characteristics between the two groups, except for triage category orange $(P=0.003), M A P(P=0.02)$ and serum LDH $(0.03)$. 


\section{Chapter 6}

\section{Biomarkers do not add to the RISE UP score in predicting mortality in older medical emergency department patients}

N. Zelis, R. Hundscheid, J. Buijs, P.W. de Leeuw, M.T.M. Raijmakers, S.M.J. van Kuijk,

P.M. Stassen

Partly published in European Journal of Internal Medicine 2020

Partly submitted 


\section{Abstract}

\section{Background}

Older emergency department (ED) patients are at high risk of mortality and it is important to predict which patients are at highest risk. Biomarkers lactate, highsensitivity cardiac Troponin T (hs-cTnT), N-terminal pro-B-type natriuretic peptide (NT-proBNP), D-dimer and procalcitonin may be able to identify those at risk. We aimed to assess the discriminatory value of these biomarkers for 30-day mortality and to test their added value to the recently validated RISE UP score.

\section{Methods}

Dutch prospective cohort study in older ( $\geq 65$ years) medical ED patients, referred for internal medicine or gastroenterology. The five biomarkers were measured upon ED arrival. Area under the curves (AUCS) and interval likelihood ratios (LRs) were calculated. Logistic regression analysis was applied to test the added value of the biomarkers to the RISE UP score.

\section{Results}

The median age of the 450 included patients was 79 years (IQR 73-95). In total, 51 (11.3\%) patients died within 30 days. The AUCs of all biomarkers for prediction of mortality were sufficient to good, with the highest AUC of 0.73 for hs-cTnT and NTproBNP. Only for the highest lactate values, the LR was high enough (29.0) to be of importance to clinical decision making, but this applied to a minority of patients. The AUC of the RISE UP score for predicting 30-day mortality was 0.85 , which only slightly improved to 0.88 when lactate, hs-cTnT and D-dimer were added.

\section{Conclusion}

Although all 5 biomarkers predict 30-day mortality in older medical ED patients, their added value to the RISE UP model is not good enough to contribute to clinical decision making. 


\section{Introduction}

Older patients ( $\geq 65$ years) who visit the emergency department (ED) are at a substantial risk of adverse outcomes including short-term mortality, intensive or medium care unit (ICU/MCU) admission, functional decline and readmissions. ${ }^{1-3}$ During the ED visit, it is crucial to establish which patients are at highest risk, but this remains a challenging task. ${ }^{4}$

Biomarkers such as lactate, high-sensitivity cardiac Troponin T (hs-cTnT), N-terminal pro-B-type natriuretic peptide (NT-proBNP), D-dimer and procalcitonin (PCT) are frequently used to identify or quantify the severity of specific diseases. They are able to detect underlying conditions or diseases that are often present in the older ED population including tissue hypoperfusion, myocardial injury, heart failure, thromboembolism and infections. Although several studies report that these markers are associated with adverse outcomes and predict short-term mortality ${ }^{5-15}$, most of these were performed in younger ED patients ${ }^{5-8,10,11,14-16}$, in selected ED patients with infection or sepsis ${ }^{10,14-16}$ or in patients with non-specific complaints. ${ }^{12}$ It is also noteworthy that in these studies biomarkers were only measured when the ED physician deemed this to be indicated. Because they were not routinely measured $^{5-8,10-12}$, the true discriminatory value of these biomarkers for predicting adverse outcomes in older ED patients remains unknown.

Recently, we developed and validated a simplified and accurate prediction model for 30-day mortality in older ED patients, the RISE UP score. ${ }^{17}$ The RISE UP score consists of six items: age, $\geq 2$ abnormal vital signs, albumin, blood urea nitrogen (BUN), LDH and bilirubin and has an AUC of 0.83. We hypothesised that the already high discriminatory value of the model might improve even further when biomarkers would be added to the RISE UP score.

The aim of this prospective study was, therefore, to assess the discriminatory value of arterial lactate, hs-cTnT, NT-proBNP, D-dimer and PCT for 30-day mortality and other adverse outcomes (ICU/MCU admission, prolonged length of hospital stay (LOS), loss of independent living and unplanned readmission) when measured routinely in older medical ED patients. In addition, we aimed to test the predictive ability of these biomarkers for 30-day mortality when added to the RISE UP score. 


\section{Materials and methods}

\section{Study design and patient selection}

This study is part of the RISE UP study, a prospective multicentre study conducted at the EDs of Zuyderland Medical Centre (MC) and Maastricht University Medical Centre+ (MUMC+) in The Netherlands. ${ }^{18}$ The RISE UP study aimed to identify predictors of adverse outcome in older ( $\geq 65$ years) medical patients in the first stage of the ED visit. The RISE UP score was developed for prediction of 30-day mortality using items which are easily and reliably retrievable at the ED. ${ }^{17}$

For this particular study, we used the data of patients who were recruited at the ED of Zuyderland MC, a large teaching hospital in the Netherlands. In all of these patients, we routinely measured biomarkers. Patients were included if they visited the ED between July 2016 and February 2017, were 65 years or older, examined and treated by an internist or gastroenterologist and if they provided written informed consent. Exclusion criteria were earlier participation in the study and inability to speak Dutch, German or English. This study was approved by the medical ethics committee of Zuyderland MC (NL55867.096.15) and registered on clinicaltrials.gov (NCT02946398).

\section{Sample collection and biomarker measurement}

At the moment of routine blood sampling at the ED, an additional arterial blood gas sample and two venous blood samples were drawn. Lactate levels were measured immediately after arterial blood sampling on the RAPIDPoint ${ }^{\circledR} 5000$ system and were available for the attending physician. Venous blood samples were centrifuged at $1800 \mathrm{~g}$ for 10 minutes and plasma was stored in a freezer by $-20^{\circ} \mathrm{C}$. D-dimer levels were measured within 4 weeks after presentation using the Sysmex ${ }^{\circledR}$ CS-2100i system. Plasma was analysed for hs-cTnT, NT-proBNP and PCT levels within 3-4 months by the Cobas ${ }^{\circledR} 8000$ modular analyser. Results of all biomarkers, except those for lactate, were blinded for all health care providers and only available to the investigators. If one of these four biomarkers were ordered by the attending physician as part of normal clinical practice, a different blood sample was analysed, and the results were reported as usual.

\section{Data collection and outcome measures}

We collected data from patients' electronical medical records. We recorded data of age, sex and living situation. In addition, we retrieved data on comorbidity according 
to the Charlson Comorbidity Index $(\mathrm{CCl})^{19}$ and triage category (using the Manchester Triage System (MTS)[20]. The following five vital signs were noted: heart rate (HR), mean arterial blood pressure (MAP), respiratory rate (RR), oxygen saturation and Glasgow Coma Scale (GCS). In addition, the following laboratory values were retrieved: 1) the 4 laboratory items of the RISE UP score (albumin, blood urea nitrogen (BUN), lactate dehydrogenase (LDH) and bilirubin) and 2) the 5 biomarkers mentioned above.

Thirty-day all-cause mortality, was used as primary endpoint for the discriminatory value of the biomarkers and for the assessment of their added value to the RISE UP score. The secondary endpoint was a composite endpoint of ICU/MCU admission, prolonged LOS ( $>7$ days), loss of independent living and unplanned readmission within 30 days after discharge.

\section{Statistical analysis}

We performed descriptive analyses of baseline characteristics, biomarker levels and outcomes on the observed data without imputation of missing values. Continuous variables are reported as means with standard deviations or medians with interquartile ranges (IQRs) and categorical variables as proportions. Comparisons between the survivor and non-survivor groups were made using unpaired-t-tests for continuous variables with Gaussian distribution, Mann-Whitney tests for continuous non-Gaussian data and Pearson's chi-square or Fisher's exact test for categorical data. We calculated the discriminatory value of the biomarkers for the primary and secondary outcome by constructing the area under the curves (AUCs) of receiver operating characteristics (ROCs) with 95\% confidence intervals (Cls) on the available data. Accuracy of the AUCs was considered excellent if between 0.9-1.0, very good if 0.8-0.9, good if 0.7-0.8, sufficient if 0.6-0.7 and bad if between $0.5-0.6 .^{21}$ We divided all biomarkers into 5 groups ranging from lowest through highest values. Next, interval likelihood ratios (LRs) and mortality percentages were calculated within these groups. We considered high LRs $(>10)$ and low LR $(<0.1)$ being of additional value to clinical decision making. ${ }^{22}$

We used univariable logistic regression to compute the Odds Ratios (ORs) with 95\% $\mathrm{Cls}$ for the biomarkers with respect to 30-day mortality. Logistic regression analyses were performed on data after imputation of missing values to allow for the inclusion of all patients in the model. Missing values of the RISE UP score and of biomarkers were imputed using stochastic regression imputation with predictive mean matching (Supplemental Table S6.1). All laboratory results were tested for collinearity using 
Pearson's correlation coefficient. The laboratory values were checked for influential outliers using Cook's distance. Linearity was visually checked for all biomarkers and log transformed or dichotomised depending on the relationship with the outcome. For dichotomisation, the optimum cut-off value was chosen based on the values being closest to the upper left corner of the AUC. If two values were equally distanced, the Youden's Index was used. Multivariable logistic regression was used to test the added value of the biomarkers to the RISE UP score. Biomarkers were first individually added to the score. Next, we added multiple biomarkers to the score selected through backward stepwise elimination. Multiple new models were constructed this way and we determined the discriminatory ability of each of these models by calculating the AUCs with $95 \% \mathrm{Cls}$.

All data were analysed using IBM SPSS Statistics for Windows, Version 24.0, IBM Corp., Armonk, N.Y., USA and $p$-values $\leq 0.05$ were considered statistically significant.

\section{Results}

\section{Patient characteristics}

For all 450 patients who were included during the study period follow up was complete. The median age of these patients was 79 years (interquartile range 73-85) and $52 \%$ was male. In total, $51(11.3 \%)$ patients died within 30 days after the ED visit and 201 (44.7\%) met the composite endpoint. Patients in the non-survivor group (median 83 years) were older than in the survivor group (median 79 years, p-value $<0.001)$. Non-survivors more frequently experienced the composite endpoint $(72.5 \%)$ compared to the survivors (41.1\%).

\section{Biomarkers}

Biomarker values were above the reference range in most patients $(66.4,86.0,78.0$ and $79.8 \%$ for hs-cTnT, NT-proBNP, D-dimer and PCT, resp.), except for lactate, which was above reference range in only $25.6 \%$ of patients. The median values of the biomarkers were higher in non-survivors than in survivors (Table 6.1; p-values of $<0.001$ for all). The highest values of the biomarkers were more often present in nonsurvivors whereas the lowest values were more often present in survivors, but there was a large overlap in biomarker values between the non-survivors and survivors (Figure 6.1). 
Table 6.1 Baseline characteristics of study participants. ${ }^{a}$

\begin{tabular}{|c|c|c|c|c|c|}
\hline & $\begin{array}{l}\text { Reference } \\
\text { Values }\end{array}$ & $\begin{array}{c}\text { Total } \\
N=450\end{array}$ & $\begin{array}{c}\text { Non-survivors } \\
\mathrm{N}=51\end{array}$ & $\begin{array}{c}\text { Survivors } \\
N=399\end{array}$ & $d$ \\
\hline Age, median (IQR), years ${ }^{c}$ & & 450 & $83(77-87)$ & 79 (73-85) & $* * *$ \\
\hline Male sex, $\mathrm{n} \%$ & & 450 & $26(51.0)$ & $208(52.1)$ & \\
\hline Community-dwelling, $n$ \% & & 450 & $36(70.6)$ & $353(88.5)$ & $* * *$ \\
\hline $\mathrm{CCl}$ score, median (IQR) & & 450 & $3(2-5)$ & $2(1-3)$ & $* *$ \\
\hline MTS category, $\mathrm{n} \%$ & & 447 & & & $*$ \\
\hline Red & & & $2(3.9)$ & $1(0.3)$ & \\
\hline Orange & & & $10(19.6)$ & 46 (11.6) & \\
\hline Yellow & & & $28(54.9)$ & $226(57.1)$ & \\
\hline Green & & & $11(21.6)$ & $122(30.8)$ & \\
\hline Blue & & & - & $1(0.3)$ & \\
\hline \multicolumn{6}{|l|}{ RISE UP score } \\
\hline$\geq 2$ abnormal vital signs ${ }^{\mathrm{b}, \mathrm{c}}, \mathrm{n} \%$ & & 440 & $32(62.7)$ & $99(25.4)$ & $* * *$ \\
\hline albumin, median (IQR), $\mathrm{g} / \mathrm{L}^{\mathrm{c}}$ & $35-50$ & 434 & $32(29-36)$ & $39(36-41)$ & $* * *$ \\
\hline BUN, median (IQR), $\mathrm{mmol} / \mathrm{L}^{\mathrm{c}}$ & 3.5-7.5 & 445 & $12.4(7.0-23.5)$ & $7.6(5.5-12.0)$ & $* *$ \\
\hline $\mathrm{LDH}$, median (IQR), $\mathrm{U} / \mathrm{L}^{\mathrm{c}}$ & $q<247$ ते<248 & 442 & $283(206-422)$ & $222(184-282)$ & $* *$ \\
\hline bilirubin $>20 \mu \mathrm{mol} / \mathrm{L}, \mathrm{n} \%^{\mathrm{c}}$ & $<21$ & 439 & $14(27.5)$ & $50(12.9)$ & $* *$ \\
\hline \multicolumn{6}{|l|}{ Biomarkers } \\
\hline lactate, median (IQR), mmol/L & $0.6-1.8$ & 378 & $2.0(1.5-2.8)$ & $1.4(1.0-1.9)$ & $* * *$ \\
\hline hs-cTnT, median (IQR), ng/L & $<14$ & 425 & $42(26-84)$ & $21(12-39)$ & $* * *$ \\
\hline NT-proBNP, median (IQR), ng/L & $<125$ & 424 & 2766 (943-11597) & 759 (266-2377) & $* * *$ \\
\hline D-dimer, median (IQR), $\mu \mathrm{g} / \mathrm{L}$ & $<500$ & 407 & 3445 (1281-6497) & $1251(660-2804)$ & $* * *$ \\
\hline $\mathrm{PCT}$, median (IQR), $\mathrm{ng} / \mathrm{mL}$ & $<0.05$ & 424 & $0.32(0.13-1.40)$ & $0.12(0.06-0.31)$ & $* * *$ \\
\hline
\end{tabular}

BUN, blood urea nitrogen; CCl, Charlson Comorbidity Index; hs-cTnT, high-senstivity cardiac Troponin T; $I Q R$, interquartile range; LDH, lactate dehydrogenase; MTS, manchester triage system; NT-proBNP, $\mathrm{N}$-terminal pro-B-type natriuretic peptide; $\mathrm{PCT}$, procalcitonin; SD, standard deviation. ${ }^{\mathrm{a}}$ non-imputed data; b $\geq 2$ abnormal vital signs: $\mathrm{HR}>90 \mathrm{bpm}, \mathrm{MAP}<70 \mathrm{mmHg}$, Respiratory Rate $>20 / \mathrm{min}, \mathrm{O}_{2}$-saturation $<95 \%$ and GCS $<15$; ${ }^{\mathrm{C}}$ Items included in the RISE UP score; ${ }^{\mathrm{d}}$ Significant difference between non-survivors and survivors with a P-value of $0.01-<0.05(*), 0.001-<0.01\left(^{* *}\right)$ or $<0.001(* * *)$.

\section{Diagnostic accuracy of the biomarkers}

The AUCs for prediction of 30-day mortality were sufficient for lactate and PCT with values of 0.68 (95\% Cl: $0.59-0.77)$ and 0.67 (95\% Cl: $0.60-0.75)$ resp. (Table 6.2). The AUCs of the other biomarkers were good with the highest AUCs for hs-cTnT and NTproBNP with a value of $0.73(95 \% \mathrm{Cl} 0.66$ to 0.80$)$ for both. The AUCs of the biomarkers for the composite endpoint were mostly sufficient, but lower than for mortality (ranging between 0.58 and 0.67 ). 


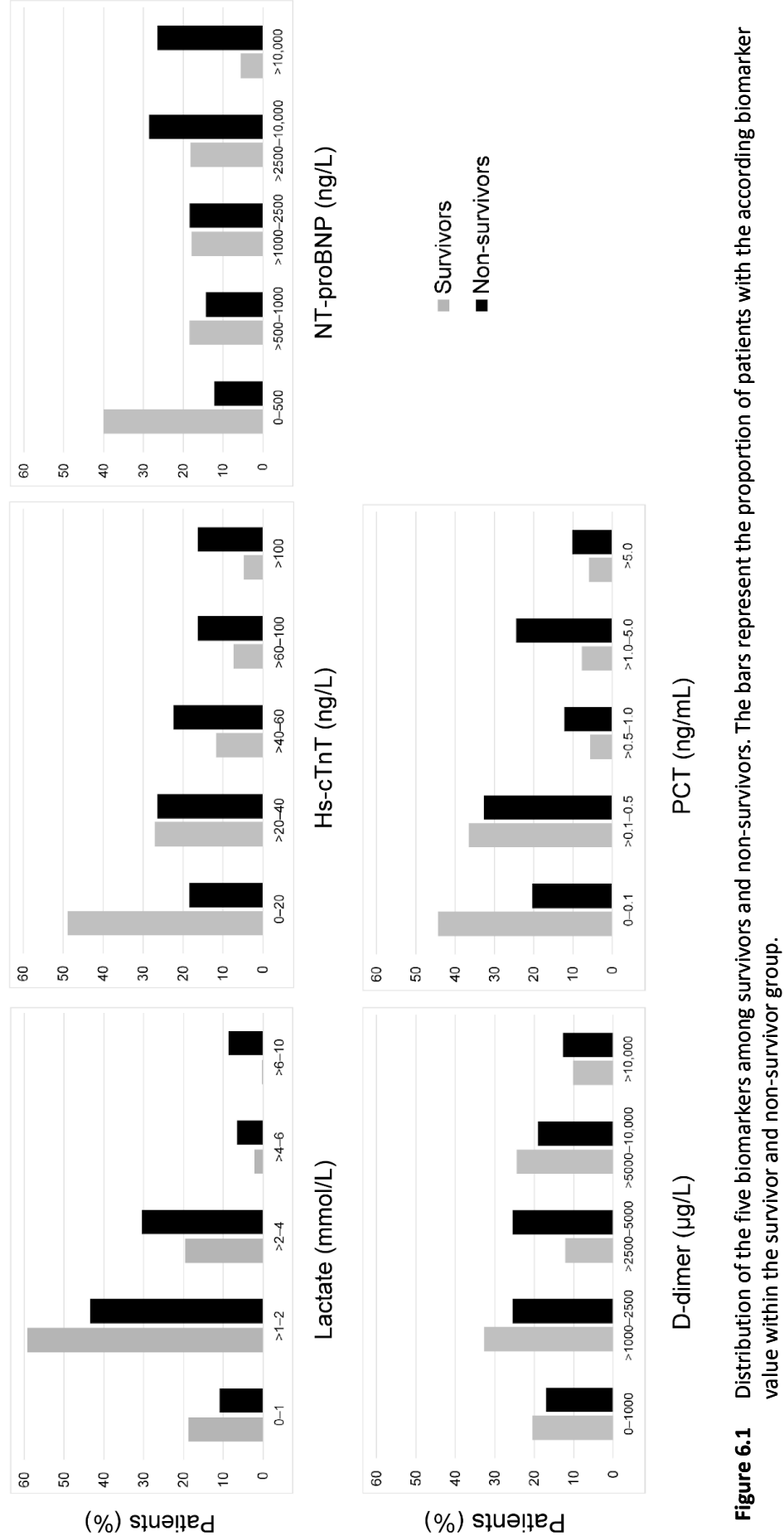


Table 6.2 AUCs for the biomarkers with respect to mortality and the composite endpoint. ${ }^{a}$

\begin{tabular}{lccccc}
\hline & & \multicolumn{4}{c}{ AUC (95 \% Cl) } \\
\cline { 3 - 6 } & $\mathbf{n}$ & \multicolumn{3}{c}{ 30-day mortality } & Composite endpoint \\
\hline Biomarker & & & & & \\
lactate & 378 & & $0.68(0.59-0.77)$ & $0.62(0.56-0.67)$ \\
hs-cTnT & 425 & $0.73(0.66-0.80)$ & $0.67(0.61-0.72)$ \\
NT-proBNP & 424 & $0.73(0.66-0.80)$ & $0.65(0.60-0.71)$ \\
D-dimer & 407 & $0.70(0.62-0.77)$ & $0.58(0.52-0.64)$ \\
PCT & 424 & $0.67(0.60-0.75)$ & $0.65(0.60-0.70)$ \\
\hline
\end{tabular}

AUC, area under the curve; $\mathrm{Cl}$, confidence interval; hs-cTnT, high-senstivity cardiac Troponin T; NT-proBNP, $\mathrm{N}$-terminal pro-B-type natriuretic peptide; $\mathrm{PCT}$, procalcitonin. ${ }^{\mathrm{a}}$ non-imputed data.

LRs increased with higher biomarker values, except for PCT (Table 6.3). Most of the biomarkers had maximum LRs between 3.2 (PCT) and 4.7 (NT-proBNP), except for lactate. We found a maximum LR of 29.0 when lactate was between 6.0 and 10.0 $\mathrm{mmol} / \mathrm{L}$ with a mortality percentage of $80.0 \%$. The maximum LRs were, however, only applicable for a limited number of patients $(n=5)$. The lowest LRs for all biomarkers were less variable but ranging between 0.3 (NT-proBNP) and 0.6 (lactate).

\section{Univariable logistic regression analysis}

Lactate and D-dimer were dichotomised, and hs-cTnT, NT-proBNP and PCT were logarithmically transformed because they were not linearly associated with 30-day mortality. The optimum cut-off value was $>1.5 \mathrm{mmol} / \mathrm{L}$ for lactate and $>3000 \mu \mathrm{g} / \mathrm{L}$ for D-dimer. None of the biomarkers were highly correlated. In the univariable logistic regression analysis, all biomarkers were strong predictors of 30-day mortality with p-values of $<0.001$ (Table 6.4). The RISE UP score was a strong predictor of 30-day mortality as well with 1.08 odds increase in mortality with every percentage point increase in predicted probability. 
Table 6.3 Interval likelihood ratios for the biomarkers.

\begin{tabular}{|c|c|c|c|c|c|}
\hline \multirow[t]{2}{*}{ Biomarker } & \multicolumn{2}{|c|}{ Mortality } & \multirow[t]{2}{*}{$\mathbf{N}$} & \multirow[t]{2}{*}{ LR } & \multirow{2}{*}{$\begin{array}{c}\text { Observed mortality } \\
\text { (\%) }\end{array}$} \\
\hline & Yes (n \%) & No (n \%) & & & \\
\hline \multicolumn{6}{|l|}{ lactate (mmol/L) } \\
\hline $0-1.0$ & 5 (10.9) & $62(18.7)$ & 67 & 0.6 & 7.5 \\
\hline$>1.0-2.0$ & $20(43.5)$ & $197(59.3)$ & 217 & 0.7 & 9.2 \\
\hline$>2.0-4.0$ & $14(30.4)$ & $65(19.6)$ & 79 & 1.6 & 17.7 \\
\hline$>4.0-6.0$ & $3(6.5)$ & $7(2.1)$ & 10 & 3.1 & 30.0 \\
\hline$>6.0-10.0$ & $4(8.7)$ & $1(0.3)$ & 5 & 29.0 & 80.0 \\
\hline \multicolumn{6}{|l|}{ hs-cTnT (ng/L) } \\
\hline $0-20$ & $9(18.4)$ & $184(48.9)$ & 193 & 0.4 & 4.7 \\
\hline$>20-40$ & $13(26.5)$ & $102(27.1)$ & 115 & 1.0 & 11.3 \\
\hline$>40-60$ & $11(22.4)$ & $44(11.7)$ & 55 & 1.9 & 20.0 \\
\hline$>60-100$ & $8(16.3)$ & $28(7.4)$ & 36 & 2.2 & 22.2 \\
\hline$>100$ & $8(16.3)$ & $18(4.8)$ & 26 & 3.4 & 30.8 \\
\hline \multicolumn{6}{|l|}{ NT-proBNP (ng/L) } \\
\hline $0-500$ & $6(12.2)$ & $150(40.0)$ & 156 & 0.3 & 3.8 \\
\hline$>500-1000$ & $7(14.3)$ & $69(18.4)$ & 76 & 0.8 & 9.2 \\
\hline$>1000-2500$ & $9(18.4)$ & 67 (17.9) & 76 & 1.0 & 11.8 \\
\hline$>2500-10,000$ & $14(28.6)$ & $68(18.1)$ & 82 & 1.6 & 17.1 \\
\hline$>10,000$ & $13(26.5)$ & $21(5.6)$ & 34 & 4.7 & 38.2 \\
\hline \multicolumn{6}{|l|}{ D-dimer ( $\mu \mathrm{g} / \mathrm{L})$} \\
\hline $0-1000$ & $8(17.0)$ & $149(41.1)$ & 157 & 0.4 & 5.1 \\
\hline$>1000-2500$ & $12(25.5)$ & $112(31.1)$ & 124 & 0.8 & 9.7 \\
\hline$>2500-5000$ & $12(25.5)$ & $56(15.6)$ & 68 & 1.6 & 17.6 \\
\hline$>5000-10,000$ & 9 (19.1) & $30(8.3)$ & 39 & 2.3 & 23.1 \\
\hline$>10,000$ & $6(12.8)$ & $13(3.6)$ & 19 & 3.6 & 31.6 \\
\hline \multicolumn{6}{|l|}{ РCT (ng/L) } \\
\hline $0-0.1$ & $10(20.4)$ & $166(44.3)$ & 176 & 0.5 & 5.7 \\
\hline$>0.1-0.5$ & $16(32.7)$ & $137(36.5)$ & 153 & 0.9 & 10.5 \\
\hline$>0.5-1.0$ & $6(12.2)$ & $21(5.6)$ & 27 & 2.2 & 22.2 \\
\hline$>1.0-5.0$ & $12(24.5)$ & $29(7.7)$ & 41 & 3.2 & 29.3 \\
\hline$>5.0$ & $5(10.2)$ & $22(5.9)$ & 27 & 1.7 & 18.5 \\
\hline
\end{tabular}

hs-cTnT, high-senstivity cardiac Troponin T; LR, likelihood ratio; NT-proBNP, N-terminal pro-B-type natriuretic peptide; PCT, procalcitonin.

Table 6.4 Interval likelihood ratios for the biomarkers.

\begin{tabular}{lcc}
\hline Predictors & \multicolumn{2}{c}{ Univariable analysis } \\
& Odds Ratio (95\% Cl) & p-value \\
\hline lactate $>1.5 \mathrm{mmol} / \mathrm{L}$ & $4.29(2.18-8.44)$ & $<0.001$ \\
hs-cTnT - per log ng/L increase & $2.36(1.70-3.27)$ & $<0.001$ \\
NT-proBNP - per log ng/L increase & $1.78(1.45-2.18)$ & $<0.001$ \\
D-dimer $>3000 \mu \mathrm{g} / \mathrm{L}$ & $2.91(1.61-5.28)$ & $<0.001$ \\
PCT - per log ng/mL increase & $1.34(1.15-1.56)$ & $<0.001$ \\
RISE UP score - per \%-point increase & $1.08(1.06-1.10)$ & $<0.001$ \\
\hline
\end{tabular}

$\mathrm{Cl}=$ confidence interval; hs-cTnT, high-senstivity cardiac Troponin $\mathrm{T}$; log, logarithm; NT-proBNP, N-terminal pro-B-type natriuretic peptide; PCT, procalcitonin. ${ }^{\text {IImputed Data. }}$ 


\section{Added value of the biomarkers to the RISE UP score}

The AUC of the RISE UP score in this cohort was 0.85 ( $95 \% \mathrm{Cl} 0.80$ to 0.91 ). When the biomarkers were individually added to the RISE UP score, we found the best improvement in AUC after adding hs-cTnT which resulted in an AUC of $0.87(95 \% \mathrm{Cl}$ 0.82 to 0.92 , Table 6.5). When lactate, hs-cTnT and D-dimer were added to the RISE UP score at the same time, the AUC improved to 0.88 (95\% $\mathrm{Cl} 0.83$ to 0.92 ).

Table 6.5 Multivariable logistic regression analysis of the RISE UP score and the additional value of biomarkers for prediction of 30-day mortality. ${ }^{a}$

\begin{tabular}{|c|c|c|c|c|c|}
\hline Model & Chi-square & df & p-value & AUC & $95 \% \mathrm{Cl}$ \\
\hline RISE UP score ${ }^{b}$ & 73.74 & 1 & $<0.001$ & 0.85 & $0.80-0.91$ \\
\hline RISE UP score + lactate $>1.5 \mathrm{mmol} / \mathrm{L}^{\mathrm{b}}$ & 80.85 & 2 & $<0.001$ & 0.86 & $0.81-0.91$ \\
\hline RISE UP score + log hs-cTnT ${ }^{b}$ & 77.54 & 2 & $<0.001$ & 0.87 & $0.82-0.92$ \\
\hline RISE UP score + log NT-proBNP ${ }^{b}$ & 76.78 & 2 & $<0.001$ & 0.86 & $0.80-0.91$ \\
\hline RISE UP score + D-dimer $>3000 \mu \mathrm{g} / \mathrm{L}^{\mathrm{b}}$ & 78.50 & 2 & $<0.001$ & 0.86 & $0.81-0.92$ \\
\hline RISE UP score $+\log \mathrm{PCT}^{\mathrm{b}}$ & 74.96 & 2 & $<0.001$ & 0.83 & $0.76-0.90$ \\
\hline $\begin{array}{l}\text { RISE UP score + lactate }>1.5 \mathrm{mmol}^{\mathrm{L}}+\mathrm{D}- \\
\text { dimer }>3000 \mu \mathrm{g} / \mathrm{L}+\log \mathrm{hs}-\mathrm{cTnT}^{\mathrm{c}}\end{array}$ & 88.56 & 4 & $<0.001$ & 0.88 & $0.83-0.92$ \\
\hline
\end{tabular}

AUC, area under the curve; $\mathrm{Cl}$, confidence interval; df, degrees of freedom; hs-cTnT, high-senstivity cardiac Troponin T; log, logarithm; NT-pro-BNP, N-terminal pro-B-type natriuretic peptide; OR, Odds ratio; PCT, procalcitonin. ${ }^{\mathrm{a}}$ Imputed Data; ${ }^{\mathrm{b}}$ Models created by adding the biomarkers individually to the RISE UP score using multivariable logistic regression analysis; ' $\mathrm{C}$ odel created by adding multiple biomarkers to the RISE UP score using backward stepwise logistic regression analysis.

\section{Discussion}

To the best of our knowledge, this is the first study evaluating the discriminatory value of lactate, hs-cTnT, NT-proBNP, D-dimer and PCT, when measured routinely, for predicting clinical outcome in older ( $\geq 65$ years) medical ED patients. We found that these 5 biomarkers were predictive of 30-day mortality with the best discriminatory values for hs-cTnT and NT-proBNP (AUCs of 0.73). However, a large overlap in biomarker values was observed between the survivor and non-survivor group, resulting in suboptimal LRs. For predicting 30-day mortality, the additional value of the biomarkers to that of the RISE UP score was only minimal, even when lactate, Ddimer and hs-cTnT were all added. Overall, the predictive ability of the biomarkers for the composite endpoint was lower than for the primary endpoint. 


\section{Predictive value of individual biomarkers}

We showed that lactate, hs-cTnT, NT-proBNP, D-dimer and PCT were sufficient to good predictors of 30-day mortality (AUCs ranging from 0.67 to 0.73 ) and sufficient predictors of the composite endpoint (AUC ranging from 0.58 to 0.67 ) in older medical ED patients. Other studies showed the same results. ${ }^{10-12,14,15,23-29}$ However, in most of these studies, biomarkers were not measured routinely. Moreover, two studies showed that mortality was are lowest in patients in whom biomarkers were not ordered during normal clinical practice. ${ }^{8,11}$ These findings show that the predictive value of biomarkers measured in all patients differs from that measured only when indicated by the physician. We think that the predictive values we found for the biomarkers are more reflective of their true prognostic ability than when measured on indication.

Despite the fact that these 5 biomarkers were overall predictive of 30-day mortality, on an individual level we found a large overlap in biomarker values between survivors and non-survivors. The overlap in biomarker values was most prominent in patients with non-extreme values. Especially in this group of patients, it is likely that the prognosis of the patient is less evident to the treating physician. Therefore, an estimation of prognosis provided by a biomarker is likely to be important. However, the discriminatory value of biomarker values in these patients was low as illustrated by the moderate LRs. In an US study in trauma patients, clinically meaningful contribution to decision making only occurred at lactate levels of $>9 \mathrm{mmol} / \mathrm{L}^{30}$, which was only present in a minority of patients. In our study, lactate had an important LR of 29 when between $6-10 \mathrm{mmol} / \mathrm{l}$, but this LR was found in only five patients. For the secondary composite endpoint, the discriminatory value of the biomarkers was even lower (ranging between 0.58 and 0.67 ). Therefore, we conclude that the five biomarkers we studied do not contribute to clinical decision making.

\section{The added value of biomarkers to the RISE UP score}

We found that the value of the five biomarkers was insignificant when added to the RISE UP score. We only found a maximum increase of 0.03 in AUC (from 0.85 to 0.88 ) once lactate, hs-cTnT and D-dimer were added at the same time. These results are at variance with the data from a Swiss study in ED patients with nonspecific complaints. $^{12}$ In that particular study, the AUC of the Katz-ADL index score improved from 0.68 to 0.80 when D-dimer was added. This increase in AUC may be explained by the relative low discriminatory value of the Katz-ADL index score (AUC of 0.68). Similar to our finding, the AUC of the MEDS score, which is used in sepsis patients, only 
minimally improved from 0.78 to 0.81 when PCT was added. ${ }^{29}$ This suggests that the increase in AUC depends on the strength of the baseline model ${ }^{31}$ and that biomarkers only slightly improve the predictive ability of risk-stratification scores that already have good discriminatory power.

Besides the lack of substantial improvement in discriminatory ability, the extra costs and loss of time for determining the biomarkers should be taken into account. All items incorporated in the RISE UP score were ordered by the physician for diagnostic reasons in $>90 \%$ of patients. Biomarkers were ordered by the physician when deemed necessary and this occurred in less than $10 \%$ of the patients, except for lactate, which was ordered in approximately $25 \%$ of the patients (Supplemental Table S6.2). The costs for routinely determining these 5 biomarkers are high as compared to the relatively cheap items used in the RISE UP score. Measuring biomarkers on a routine basis will therefore lead to direct extra costs, but may also serve as an incentive to perform extra diagnostic procedures in patients with high values of these biomarkers (e.g. CT pulmonary angiography or echocardiography). The high expected costs in combination with the minor improvement in discriminatory ability make us conclude that routine determination of biomarkers in older ED patients should not be advocated for prediction of 30-day mortality, not the least because the easily applicable RISE UP score provides an inexpensive assessment of mortality risk in these patients.

\section{Study strength, limitations and future perspectives}

The strengths of this study are its prospective design and the routine, i.e. unselected, measurement of these biomarkers in our cohort. Some limitations apply to this study. At first, we could not include every possible candidate, as physicians had to give priority to providing emergency care in the often crowded ED. We detected no evidence for selection bias but cannot exclude it either. ${ }^{17}$ Secondly, we only measured biomarker values immediately after arrival at the ED. It is possible that serial biomarker measurement could have yielded different information and a different predictive ability.

While the RISE UP score and the biomarkers are predictors of 30-day mortality, we do not know whether knowledge of prognosis will indeed influence clinical decision making. It is possible that this knowledge improves decision making and/or lead to improvement of outcome, well-being, length of stay in the ED and/or medical costs. Therefore, the impact of prediction models on clinical practice and patient related 
outcome measures will be an important subject for future studies. In addition, the prognostic value of these biomarkers in predicting other outcomes such as major adverse cardiovascular events or cancer can be interesting subjects for future research.

\section{Conclusions}

Lactate, hs-cTnT, NT-proBNP, D-dimer and PCT, when measured routinely, show a predictive value with regard to short-term mortality and other adverse outcomes in older medical ED patients, but, given the large overlap in values between those with and without adverse outcomes and their minimal added value to the RISE UP score, they are unlikely to contribute to clinical decision making. Their routine measurement is, in our opinion, therefore not recommended, especially because the feasible RISE UP score provides an inexpensive assessment of mortality risk in these patients.

\section{Acknowledgements}

We thank the medical and nursing staff of the emergency departments of Zuyderland Medical Centre and Maastricht University Medical Centre+ for their contribution to our study and most of all, all patients who participated in the study. 


\section{References}

1. Aminzadeh F, Dalziel WB. Older adults in the emergency department: a systematic review of patterns of use, adverse outcomes, and effectiveness of interventions. Ann Emerg Med. 2002;39:238-47.

2. Samaras N, Chevalley T, Samaras D, Gold G. Older patients in the emergency department: a review. Ann Emerg Med. 2010;56:261-9.

3. Salvi F, Morichi V, Grilli A, Giorgi R, De Tommaso G, Dessi-Fulgheri P. The elderly in the emergency department: a critical review of problems and solutions. Intern Emerg Med. 2007;2:292-301.

4. Carpenter CR, Shelton E, Fowler S, Suffoletto B, Platts-Mills TF, Rothman RE, et al. Risk factors and screening instruments to predict adverse outcomes for undifferentiated older emergency department patients: a systematic review and meta-analysis. Acad Emerg Med. 2015;22:1-21.

5. Barfod C, Lundstrom LH, Lauritzen MM, Danker JK, Soletormos G, Forberg JL, et al. Peripheral venous lactate at admission is associated with in-hospital mortality, a prospective cohort study. Acta Anaesth Scand. 2015;59:514-23.

6. Datta D, Walker C, Gray AJ, Graham C. Arterial lactate levels in an emergency department are associated with mortality: a prospective observational cohort study. Emerg Med J. 2015;32:673-7.

7. Pedersen M, Brandt VS, Holler JG, Lassen AT. Lactate level, aetiology and mortality of adult patients in an emergency department: a cohort study. Emerg Med J. 2015;32:678-84.

8. van den Nouland DP, Brouwers MC, Stassen PM. Prognostic value of plasma lactate levels in a retrospective cohort presenting at a university hospital emergency department. BMJ Open. 2017;7:e011450.

9. Bahrmann P, Christ M, Hofner B, Bahrmann A, Achenbach S, Sieber CC, et al. Prognostic value of different biomarkers for cardiovascular death in unselected older patients in the emergency department. Eur Heart J Acute Cardiovasc Care. 2016;5:568-78.

10. de Groot B, Verdoorn RC, Lameijer J, van der Velden J. High-sensitivity cardiac troponin T is an independent predictor of inhospital mortality in emergency department patients with suspected infection: a prospective observational derivation study. Emerg Med J. 2014;31:882-8.

11. Courtney D, Conway R, Kavanagh J, O'Riordan D, Silke B. High-sensitivity troponin as an outcome predictor in acute medical admissions. Postgrad Med J. 2014;90:311-6.

12. Nickel CH, Kuster T, Keil C, Messmer AS, Geigy N, Bingisser R. Risk stratification using D-dimers in patients presenting to the emergency department with nonspecific complaints. Eur J Intern Med. 2016;31:20-4.

13. Luchner A, Mockel M, Spanuth E, Mocks J, Peetz D, Baum H, et al. N-terminal pro brain natriuretic peptide in the management of patients in the medical emergency department (PROMPT): correlation with disease severity, utilization of hospital resources, and prognosis in a large, prospective, randomized multicentre trial. Eur J Heart Fail. 2012;14:259-67.

14. Rodelo JR, De la Rosa G, Valencia ML, Ospina S, Arango CM, Gomez Cl, et al. D-dimer is a significant prognostic factor in patients with suspected infection and sepsis. Am J Emerg Med. 2012;30:1991-9.

15. Wilhelm J, Hettwer S, Hammer D, Schurmann M, Christoph A, Amoury M, et al. Outcome prediction using clinical scores and biomarkers in patients with presumed severe infection in the emergency department. Med Klin Intensivmed Notfmed. 2012;107:558-63.

16. Freund $Y$, Delerme S, Goulet H, Bernard M, Riou B, Hausfater P. Serum lactate and procalcitonin measurements in emergency room for the diagnosis and risk-stratification of patients with suspected infection. Biomarkers. 2012;17:590-6.

17. Zelis N, Buijs J, de Leeuw PW, van Kuijk SMJ, Stassen PM. A new simplified model for predicting 30day mortality in older medical emergency department patients: The rise up score. Eur J Intern Med. 2020;77:36-43.

18. Zelis N, Buijs J, de Leeuw PW, van Kuijk SMJ, Stassen PM. Study protocol for a multicentre prospective cohort study to identify predictors of adverse outcome in older medical emergency department patients (the Risk Stratification in the Emergency Department in Acutely Ill Older Patients (RISE UP) study). BMC Geriatr. 2019;19:65. 
19. Charlson ME, Pompei P, Ales KL, MacKenzie CR. A new method of classifying prognostic comorbidity in longitudinal studies: development and validation. J Chronic Dis. 1987;40:373-83.

20. K M-J. Emergency triage. London: BMJ Publishing Group 1997; 1997.

21. Simundic AM. Measures of Diagnostic Accuracy: Basic Definitions. Ejifcc. 2009;19:203-11.

22. Grimes DA, Schulz KF. Refining clinical diagnosis with likelihood ratios. Lancet (London, England). 2005;365:1500-5.

23. Kruse O, Grunnet N, Barfod C. Blood lactate as a predictor for in-hospital mortality in patients admitted acutely to hospital: a systematic review. Scand J Trauma Resusc Emerg Med. 2011;19:74.

24. Park YJ, Kim DH, Kim SC, Kim TY, Kang C, Lee SH, et al. Serum lactate upon emergency department arrival as a predictor of 30-day in-hospital mortality in an unselected population. PloS One. 2018;13:e0190519.

25. Puskarich MA, Illich BM, Jones AE. Prognosis of emergency department patients with suspected infection and intermediate lactate levels: a systematic review. J Crit Care. 2014;29:334-9.

26. del Portal DA, Shofer F, Mikkelsen ME, Dorsey PJ, Jr., Gaieski DF, Goyal M, et al. Emergency department lactate is associated with mortality in older adults admitted with and without infections. Acad Emerg Med. 2010;17:260-8.

27. Marchetti M, Benedetti A, Mimoz O, Lardeur JY, Guenezan J, Marjanovic N. Predictors of 30-day mortality in patients admitted to ED for acute heart failure. Am J Emerg Med. 2017;35:444-7.

28. Meyer B, Huelsmann M, Wexberg P, Delle Karth G, Berger R, Moertl D, et al. N-terminal pro-B-type natriuretic peptide is an independent predictor of outcome in an unselected cohort of critically ill patients. Crit Care Med. 2007;35:2268-73.

29. Zhao Y, Li C, Jia Y. Evaluation of the Mortality in Emergency Department Sepsis score combined with procalcitonin in septic patients. Am J Emerg Med. 2013;31:1086-91.

30. Baron BJ, Nguyen A, Stefanov D, Shetty A, Zehtabchi S. Clinical value of triage lactate in risk stratifying trauma patients using interval likelihood ratios. Am J Emerg Med. 2018;36:784-8.

31. Pencina MJ, D'Agostino RB, Pencina KM, Janssens AC, Greenland P. Interpreting incremental value of markers added to risk prediction models. Am J Epidemiol. 2012;176:473-81. 


\section{Supplemental data}

Table S6.1 Overview imputed values.

\begin{tabular}{lc}
\hline Imputed variable & $\begin{array}{c}\text { Total } \\
(\mathbf{n = 4 5 0 )} \\
\mathbf{n}(\%)\end{array}$ \\
\hline Heart rate & $3(0.7)$ \\
MAP & $13(2.9)$ \\
Oxygen saturation & $10(2.2)$ \\
Albumin & $16(3.6)$ \\
BUN & $5(1.1)$ \\
LDH & $8(1.8)$ \\
Bilirubin & $11(2.4)$ \\
Lactate & $72(16.0)$ \\
Hs-cTnT & $25(5.6)$ \\
NT-proBNP & $26(5.8)$ \\
D-dimer & $43(9.6)$ \\
PCT & $26(5.8)$ \\
\hline
\end{tabular}

BUN, blood urea nitrogen; Hs-cTnT, high-sensitivity cardiac Troponin T; LDH, lactate dehydrogenase; MAP, mean arterial pressure; NT-proBNP, N-terminal pro-B-type natriuretic peptide; PCT, procalcitonin.

Table S6.2 Laboratory tests ordered by the physician.

\begin{tabular}{lc}
\hline Laboratory tests & \% of patients $^{\mathbf{a}}$ \\
\hline RISE UP score & \\
Albumin & 96.4 \\
BUN & 98.9 \\
LDH & 98.2 \\
Bilirubin & 97.6 \\
Biomarkers & \\
Lactate & 25.8 \\
Hs-cTnT & 7.6 \\
NT-proBNP & 8.7 \\
D-dimer & 5.1 \\
PCT & 0.0 \\
\hline
\end{tabular}

BUN, blood urea nitrogen; Hs-cTnT, high-senstivity cardiac Troponin T; LDH, lactate dehydrogenase; NT-pro$\mathrm{BNP}, \mathrm{N}$-terminal pro-B-type natriuretic peptide; $\mathrm{PCT}$, procalcitonin. ${ }^{\mathrm{a}}$ Represents the proportion of patients were the laboratory test was ordered by the physician. 

Chapter 7

\section{Major adverse cardiovascular events in older emergency department patients presenting with non-cardiac medical complaints}

N. Zelis, A.M.M. Roumans-van Oijen, J. Buijs, D.J.W. van Kraaij, S.M.J. van Kuijk,

P.W. de Leeuw, P.M. Stassen 


\section{Abstract}

\section{Introduction}

The risk of major adverse cardiovascular events (MACE) for older emergency department (ED) patients who present with non-cardiac medical complaints is unknown. Early identification of patients at risk of MACE is highly important to be able to apply preventive measures timely. Biomarkers may play a role in this early identification. We aimed at investigating the incidence of MACE, the predictive value of high-sensitivity cardiac Troponin T (hs-cTnT) and N-terminal pro-B-type natriuretic peptide (NT-proBNP) for subsequent MACE, and the incidence of all-cause and cardiovascular (CV) mortality within 1 year after ED visit.

\section{Methods}

The current paper describes a substudy of a large Dutch prospective cohort study (RISE UP study) in older ( $\geq 65$ years) medical ED patients who presented with noncardiac complaints. Biomarkers were measured upon ED arrival. Cox-regression analysis was used to determine the predictive value of the biomarkers, when corrected for other possible predictors of MACE, and area under the curves (AUCs) were calculated.

\section{Results}

Of 431 patients with a median age of 79 years, 86 (20.0\%) developed a MACE within 1 year after their index visit. Both hs-cTnT and NT-proBNP were predictive of MACE with an AUC of $0.74(0.68-0.80)$ for both, and a hazard ratio (HR) of $2.00(95 \% \mathrm{Cl} 1.68$ 2.39) and 1.82 (95\% Cl 1.57-2.11), resp. Multivariable analysis correcting for other possible predictors of MACE revealed NT-proBNP as an independent predictor of MACE. In the total population, 32.6\% died within 1 year after the ED visit. Of these deaths, $15.1 \%$ were certainly due to CV causes.

\section{Conclusion}

Older medical ED patients are at high risk of subsequent MACE and dying within 1 year after their ED visit. While both hs-cTnT and NT-proBNP are predictive, only NT-proBNP is an independent predictor of MACE. It is likely that early identification of those at risk offers a window of opportunity for prevention. 


\section{Introduction}

Older patients are at increased risk of adverse outcomes after an emergency department (ED) visit. ${ }^{1,2}$ Despite the fact that cardiovascular disease (CVD) still remains the leading cause of death worldwide, the risk of MACE for older ED patients, presenting with non-cardiac medical complaints, is unknown. Because preventive measures may improve outcome ${ }^{3}$, early identification of older patients at risk of MACE is highly important.

Besides conventional cardiovascular (CV) risk factors, a presentation with infection, ECG abnormalities and renal function are predictive of MACE. ${ }^{3-7}$ In particular, the cardiac biomarkers high-sensitivity cardiac Troponin T (hs-cTnT) and N-terminal pro-Btype natriuretic peptide (NT-proBNP), have also been shown to be predictors of MACE. ${ }^{8-12}$ Hs-cTnT elevates after cardiomyocyte necrosis and is extremely useful in diagnosing acute myocardial infarction. NT-proBNP is secreted by the ventricles of the heart in response to increased ventricular filling pressure and, when elevated, it may be a marker of heart failure. ${ }^{13,14}$ The incidence of MACE in older patients presenting to the ED with non-cardiac, medical complaints is, however, unknown. In addition, the predictive value of hs-cTnT and NT-proBNP for MACE in this population has not yet been studied.

We hypothesised that patients who present to the ED with non-cardiac complaints are at high risk of developing MACE within 1 year and that both NT-proBNP and troponin $T$ are independent predictors of subsequent MACE in this population. In this prospective study, we investigated the incidence of MACE within 1 year after ED visit in older ED patients who present with non-cardiac medical complaints. In addition, we investigated the discriminatory value of both hs-cTnT and NT-proBNP for subsequent MACE and the incidence of 1-year all-cause and CV mortality in this cohort of older patients.

\section{Methods}

\section{Study design and patient selection}

This study is part of the RISE UP study, a prospective multicentre observational cohort study, which aimed to identify predictors of adverse outcome in older ED patients. ${ }^{15}$ For the current study, a secondary analysis was performed on data of patients 
included in one of the two participating hospitals (Zuyderland $M C$ ), since biomarkers were routinely assessed in all participants at this site. Zuyderland $\mathrm{MC}$ is a secondary care teaching hospital in the south of the Netherlands with 635 beds and 30,000 ED visits annually. Patients who present with cardiac complaints are assessed and treated at a heart aid unit, which is located elsewhere in the hospital.

Patients were included if they were 65 years or older, were assessed by an internist or gastroenterologist and provided written informed consent. Patients were excluded if they already participated, were unable to speak Dutch, German or English or when a MACE was already present at the time of their index visit. All patients were followed for 1 year.

This study was approved by the medical ethics committee of Zuyderland MC (NL55867.096.15) and registered on clinicaltrials.gov (NCT02946398). This study was reported according to the strengthening the reporting of observational studies in epidemiology (STROBE) guidelines. ${ }^{16}$

\section{Biomarker measurement}

During routine blood sampling at the beginning of the ED visit, one additional venous blood sample was drawn. This blood sample was centrifuged at $1800 \mathrm{~g}$ for 10 minutes and plasma was stored in a freezer by $-20^{\circ} \mathrm{C}$ for a maximum period of 4 months. Plasma was analysed for hs-cTnT and NT-proBNP by the Cobas ${ }^{\circledR} 8000$ modular analyser. Results were only available to the investigators and blinded for all health care providers, unless they specifically requested these biomarkers. In that case, a different blood sample was analysed and results were reported as usual. Therefore, medical care was not influenced by this study.

\section{Data collection}

Data were retrieved from electronical medical records. We collected the following possible predictors of MACE: age, sex, CV comorbidities, CV risk factors, laboratory tests and electrocardiograms (ECGs). In more detail, the following CV comorbidities were retrieved: atrial fibrillation, cerebrovascular accident (CVA), transient ischaemic attack (TIA), peripheral vascular disease, myocardial infarction and heart failure according to the Charlson Comorbidity Index (CCI). ${ }^{17}$ In addition, we retrieved data on CV risk factors: hypertension, hypercholesterolemia, diabetes mellitus, obesity (body mass index (BMI) $>30 \mathrm{~kg} / \mathrm{m}^{2}$ ), current smoking status, and a family history of CVD. We retrieved data regarding the use of blood glucose lowering drugs, including insulin, 
antihypertensive drugs and statins. Diabetes mellitus was considered to be present when patients were treated with blood glucose lowering drugs and hypercholesterolemia when statins were used. If data regarding CV risk factors were lacking, these risk factors were assumed to be absent. The main reason for the EDvisit was categorised according to the International Classification of Diseases (ICD)-10. ${ }^{18}$ The following laboratory tests were collected: serum creatinine (from which renal function was calculated using the Modification of Diet in Renal Disease (MDRD) formula), C-reactive protein (CRP), hs-cTnT and NT-proBNP. In addition, ECGs made during the ED visit were collected and analysed by an experienced cardiologist using the criteria of the Simplified ECG score. ${ }^{4}$ This score is calculated by counting the number of abnormalities resulting in a score ranging from 0 to 12 points. An ECG was considered abnormal if the simplified ECG score was more than 0.

Last, data on MACE and mortality occurring during the one-year follow up period were collected. MACE was defined as the first manifestation of cardiac arrest, myocardial infarction, arrhythmia requiring intervention (including electric cardioversion, electrophysiological intervention or pacemaker implantation, but excluding the use of anti-arrhythmic medication), ischemic or haemorrhagic stroke, TIA, heart failure, cardiac and peripheral revascularization procedures or death attributable to a CV cause. These were derived from medical records, by checking municipal administrational records, and, if data were inconclusive, by contacting the general practitioner (GP) for additional information.

\section{Outcome measures}

The primary endpoint was the incidence of a subsequent MACE within 1 year. Secondary endpoints were 1-year all-cause mortality and CV mortality. All patients were followed up for 1 year.

\section{Data analysis}

We performed descriptive analyses of characteristics of patients with and without MACE. Continuous variables were reported as medians with interquartile ranges (IQRs) and categorical variables as proportions. For calculating the percentage of patients with elevated biomarker values, we used the laboratory reference value for hs-cTnT and the age-dependent reference value for NT-proBNP. ${ }^{19}$

We calculated area under the curves (AUCs) for receiver operating characteristics for hs-cTnT and NT-proBNP. Kaplan Meier survival curves and log-rank tests were used to analyse differences in time to first MACE for both hs-cTnT and NT-proBNP, after 
dividing them into tertiles, and to test for differences in one-year all-cause mortality between patients with and without MACE.

Possible predictors of MACE including the two biomarkers were entered in an univariate Cox regression analysis and hazard ratios (HRs) with $95 \%$ confidence intervals (Cls) were calculated. For Cox-regression analyses, all missing items were imputed using stochastic regression with predictive mean matching (Table S7.1). All variables were tested for the proportional hazards assumption by testing the influence of a time dependent Cox model and for collinearity using the Pearson's correlation coefficient. Continuous variables (age, MDRD, hs-cTnT and NT-proBNP) were used linearly or log transformed when indicated.

In the multivariable analysis, we corrected NT-proBNP and hs-cTnT for following possible predictors of MACE: sex, age, atrial fibrillation, CVA/TIA, peripheral vascular disease, myocardial infarction, heart failure, hypertension, hypercholesterolemia, diabetes mellitus, BMI>30, current smoking, a family history of CVD, a presentation with infection, ECG abnormalities, MDRD and the other biomarker (i.e. either hs-cTnT or NT-proBNP).

P-values below 0.05 were considered statistically significant for all statistical tests. All data were analysed using SPSS Statistics for Windows version 24.0 (IBM Corp., Armonk, N.Y., USA).

\section{Results}

\section{Study population and patient characteristics}

A total of 450 patients was included in the RISE UP study ${ }^{20}$ in Zuyderland MC (Supplemental Figure S7.1). In 19 patients, a MACE was already present at the time of ED visit and these patients were excluded from our analysis. The remaining 431 patients had a median age of 79 (IQR 73-85) years and $53.1 \%$ was male (Table 7.1 ). Slightly more than half of the patients had at least one CV comorbidity (52.4\%) and most had at least one CV risk factor (85.3\%). The main reasons for ED visit were infectious diseases and diseases of the digestive system. Most patients (60.4\%) had an abnormal ECG, and hs-cTnT and NT-proBNP values were above reference range in $69.5 \%$ and $34.8 \%$ of the patients, resp. Follow-up was complete for all patients. When comparing the baseline characteristics of included and non-included patients, we found no relevant differences in baseline characteristics and outcome (Supplemental Table S7.1). 
Table 7.1 Baseline characteristics of study participants.

\begin{tabular}{|c|c|c|c|}
\hline Characteristic & Reference values & $\mathbf{N}$ & $\begin{array}{c}\text { Total } \\
\mathrm{n}=431\end{array}$ \\
\hline \multicolumn{4}{|l|}{ Demographic } \\
\hline Age, median (IQR) & & 431 & $79(73-85)$ \\
\hline Sex, male & & 431 & $229(53.1)$ \\
\hline \multicolumn{4}{|l|}{ CV comorbidity } \\
\hline Atrial fibrillation & & 431 & $123(28.5)$ \\
\hline CVA/TIA & & 431 & $84(19.5)$ \\
\hline Peripheral vascular disease & & 431 & $76(17.6)$ \\
\hline Myocardial infarction & & 431 & $52(12.1)$ \\
\hline Heart failure & & 431 & $40(9.3)$ \\
\hline At least $1 \mathrm{CV}$ comorbidity $^{\mathrm{a}}$ & & 431 & $226(52.4)$ \\
\hline \multicolumn{4}{|l|}{ Cardiovascular risk factors } \\
\hline Hypertension & & 431 & $220(51.0)$ \\
\hline Hypercholesterolemia & & 431 & $212(49.2)$ \\
\hline Diabetes Mellitus & & 431 & $114(26.5)$ \\
\hline $\mathrm{BMI}>30$ & & 407 & $88(21.6)$ \\
\hline Current smoking & & 431 & $54(12.5)$ \\
\hline Family history of CVD & & 431 & $30(7.0)$ \\
\hline At least one CV risk factor ${ }^{b}$ & & 428 & $365(85.3)$ \\
\hline Reason for ED-visit (ICD-10) & & 431 & \\
\hline Infectious and parasitic disease & & & $127(29.5)$ \\
\hline Diseases of the digestive system & & & $124(28.8)$ \\
\hline Neoplasms & & & $44(10.2)$ \\
\hline Endocrine and metabolic diseases & & & $25(5.8)$ \\
\hline Diseases of the circulatory system ${ }^{c}$ & & & $24(5.6)$ \\
\hline Diseases of the respiratory system & & & $23(5.3)$ \\
\hline Diseases of blood and blood-forming organs & & & $19(4.4)$ \\
\hline Diseases of the genitourinary system & & & $19(4.4)$ \\
\hline Miscellaneous & & & $26(6.0)$ \\
\hline \multicolumn{4}{|l|}{ ECG } \\
\hline Simplified ECG score, median (IQR) & & 331 & $1(0-2)$ \\
\hline Simplified ECG score $>0$ & & 331 & $200(60.4)$ \\
\hline \multicolumn{4}{|l|}{ Routine laboratory results } \\
\hline MDRD, mL/min/1.73 m², median (IQR) & $>90$ & 428 & $56(39-75)$ \\
\hline $\mathrm{CRP}, \mathrm{mg} / \mathrm{L}$, median (IQR) & $<10$ & 427 & $30(7-88)$ \\
\hline \multicolumn{4}{|l|}{ Biomarkers } \\
\hline hs-cTnT, ng/L, median (IQR) & $<14$ & 406 & $22(13-41)$ \\
\hline hs-cTnT above reference range & $\geq 14$ & 406 & $282(69.5)$ \\
\hline NT-proBNP, ng/L, median (IQR) & $<125$ & 405 & $786(288-2521)$ \\
\hline NT-proBNP above age-adjusted limit ${ }^{d}$ & Age adjusted & 405 & $141(34.8)$ \\
\hline
\end{tabular}

BMI, body mass index; CVD, cardiovascular disease, CVA, cerebrovascular accident; CRP, C-reactive protein; ECG, electrocardiogram; hs-cTnT, high-sensitivity cardiac Troponin T,; ICD-10, International Classification of Diseases-10; IQR, interquartile range; MACE, major adverse cardiovascular events; MDRD, Modification of Diet in Renal Disease; NT-proBNP, N-terminal pro-B-type natriuretic peptide; TIA, transient ischaemic attack. Values are $n(\%)$ unless stated otherwise. ${ }^{a}$ Defined as at least $\geq 1$ of following CV comorbidities: atrial fibrillation, CVA/TIA, peripheral vascular disease, myocardial infarction and/or heart failure; ${ }^{b}$ Defined as at least $\geq 1$ of following CV risk factors: hypertension, hypercholesterolemia, diabetes mellitus , BMI >30, current smoking and/or a family history of CVD; ' Circulatory diseases that are not considered MACE (e.g. venous thromboembolisms); ${ }^{d}$ For heart failure, limits are age dependent: 50-75y: $<900 \mathrm{ng} / \mathrm{L}$; $>75$ y $<1800 \mathrm{ng} / \mathrm{L}$ 
In total, 86 patients $(20.0 \%)$ developed a MACE within 1 year after their ED visit. The median time to the occurrence of MACE was 50 days (IQR of 6 to 160 days) (Table 7.2). Heart failure (52.3\%) and CVA/TIA (26.7\%) were the most prevalent first presentations of MACE.

Table 7.2 Outcomes.

\begin{tabular}{lc}
\hline Outcomes & All patients \\
& $\mathbf{n = 4 3 1}$ \\
\hline MACE & $86(20.0)$ \\
Type of MACE & \\
Heart failure & $45(52.3)$ \\
CVA/TIA & $23(26.7)$ \\
Myocardial infarction & $5(5.8)$ \\
Revascularisation & $4(4.7)$ \\
Cardiovascular death & $4(4.7)$ \\
Arrhythmia with intervention & $3(3.5)$ \\
Asystole & $2(2.3)$ \\
Time to MACE, days, median (IQR) & $50(6-160)$ \\
All-cause 1-year mortality & $139(32.3)$ \\
Time to death, days, median (IQR) & $72(20-167)$ \\
CV mortality & $21(15.1)^{\mathrm{b}}$ \\
Time to death from CV causes, days, median (IQR) & $82(26-293)$ \\
\hline
\end{tabular}

CV, cardiovascular; CVA, cerebrovascular accident; MACE, major adverse cardiovascular events; TIA, transient ischaemic attack. Values are $n(\%)$ unless stated otherwise. ${ }^{a}$ Denominator for the types of MACE is the total number of MACE $(n=86)$; ${ }^{b}$ Denominator for CV mortality is number of all-cause mortality $(n=139)$.

\section{Discriminatory value of hs-cTnT and NT-proBNP with regard to MACE}

The AUCs of both hs-cTnT and NT-proBNP for MACE were $0.74(0.68-0.80)$. The Kaplan Meier curves (Figure 7.1a, 7.1b) showed that most MACEs occurred in patients with biomarker levels in the $3^{\text {rd }}$ tertile, and least in the $1^{\text {st }}$ tertile $(p$-value $<0.001)$.

\section{Cox-regression analysis}

For none of the included variables, the Cox proportional hazard assumption was violated and none were highly correlated. Biomarkers hs-cTnT and NT-proBNP were logarithmically transformed and age, the Simplified ECG score and MDRD were used linearly in the analysis. In the univariable analysis, hs-cTnT and NTproBNP were both predictive of a subsequent MACE with a HR of 2.00 (95\% Cl 1.68-2.39) and 1.82 (95\% $\mathrm{Cl}$ 1.57-2.11), resp. (Table 7.3). CV risk factors, age, atrial fibrillation, peripheral vascular disease, congestive heart failure, hypertension, diabetes mellitus, smoking, a family history of CVD, presentation with infection, the Simplified ECG score and the MDRD were predictive of MACE as well. 
In the multivariable analysis, NTproBNP was independently associated with MACE with a HR of 1.35 (95\% Cl $1.09-1.67)$, while hs-cTnT was not (Table 7.3).
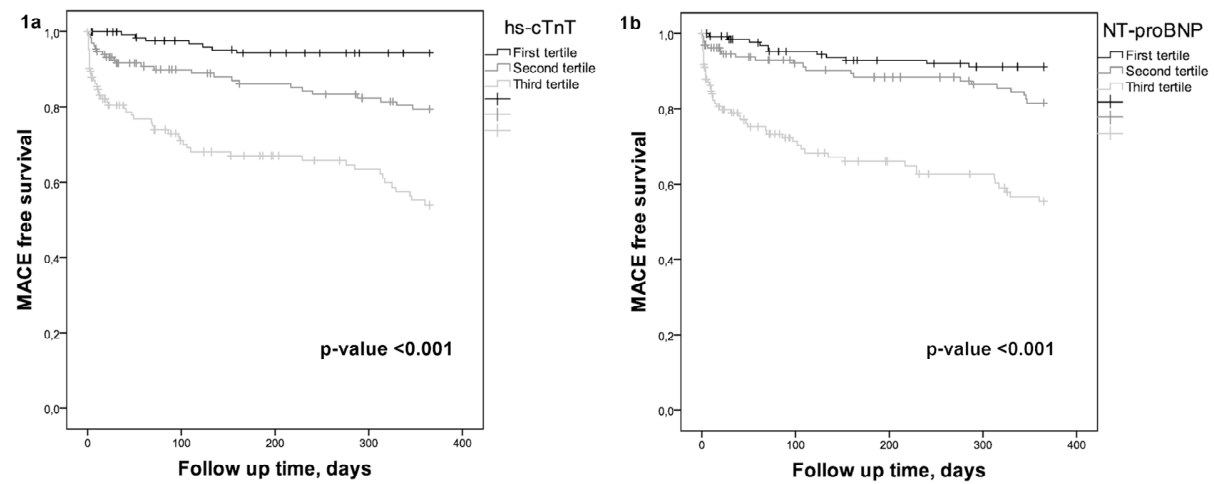

Figure 7.1 Kaplan Meier curves of MACE-free survival. Kaplan Meier curves of MACE-free survival according to the tertiles of hs-cTnT (Figure 7.1a) and NT-proBNP (Figure 7.1b).

Table 7.3. Univariable en multivariable cox regression analysis for MACE.

\begin{tabular}{|c|c|c|c|c|}
\hline \multirow[t]{2}{*}{ Predictors } & \multicolumn{2}{|c|}{ Univariable Cox-regression } & \multicolumn{2}{|c|}{ Multivariable Cox-regression } \\
\hline & $\mathrm{HR}(95 \% \mathrm{Cl})$ & p-value & Corrected HR $(95 \% \mathrm{Cl})$ & p-value \\
\hline Age - per year & $1.07(1.04-1.10)$ & $<0.001$ & & \\
\hline Male & $1.31(0.85-2.01)$ & 0.224 & & \\
\hline \multicolumn{5}{|l|}{ CV comorbidity and risk factors } \\
\hline Atrial fibrillation & $2.80(1.83-4.27)$ & $<0.001$ & & \\
\hline CVA/TIA & $1.23(0.74-4.04)$ & 0.430 & & \\
\hline Peripheral vascular disease & $1.68(1.03-2.72)$ & 0.037 & & \\
\hline Myocardial infarction & $1.47(0.83-2.60)$ & 0.189 & & \\
\hline Heart failure & $4.63(2.85-7.54)$ & $<0.001$ & & \\
\hline Hypertension & $1.66(1.07-2.56)$ & 0.023 & & \\
\hline Hypercholesterolemia & $0.72(0.47-1.10)$ & 0.130 & & \\
\hline Diabetes Mellitus & $1.72(1.11-2.67)$ & 0.016 & & \\
\hline $\mathrm{BMI}>30$ & $0.79(0.46-1.34)$ & 0.376 & & \\
\hline Current smoking & $1.13(0.61-2.07)$ & 0.700 & & \\
\hline Family history with CVD & $2.00(1.06-3.77)$ & 0.032 & & \\
\hline Presentation with infection & $1.56(1.01-2.41)$ & 0.044 & & \\
\hline Simplified ECG score - per point & $1.46(1.27-1.68)$ & $<0.001$ & & \\
\hline MDRD - per $\mathrm{mL} / \mathrm{min} / 1.73 \mathrm{~m}^{2}$ & $0.97(0.96-0.98)$ & $<0.001$ & & \\
\hline hs-cTnT - per log ng/L & $2.00(1.68-2.39)$ & $<0.001$ & $1.05(0.77-1.44)^{\mathrm{a}}$ & 0.742 \\
\hline NT-proBNP - per log ng/L & $1.82(1.57-2.11)$ & $<0.001$ & $1.35(1.09-1.67)^{\mathrm{a}}$ & 0.006 \\
\hline
\end{tabular}

$\mathrm{BMI}$, body mass index; CVD, cardiovascular disease; CVA, cerebrovascular accident; ECG, electrocardiogram; hs-cTnT, high-sensitivity cardiac Troponin T; HR, hazard ratio; MACE, major adverse cardiovascular events; MDRD, Modification of Diet in Renal Disease; NT-proBNP, N-terminal pro-B-type natriuretic peptide; TIA, transient ischaemic attack. ${ }^{\mathrm{a} C o r r e c t e d}$ for covariates: age, sex, atrial fibrillation, CVA/TIA, peripheral vascular disease, myocardial infarction, heart failure, hypertension, hypercholesterolemia, diabetes mellitus, BMI>30, current smoking, family history of CVD, presentation with infection, Simplified ECG score, MDRD and either NT-proBNP or hs-cTnT. 


\section{1-year all-cause and CV mortality}

In total, 139 patients (32.3\%) died within 1 year of the ED visit (Table 7.2) of whom 21 (of $139 ; 15.1 \%$ ) patients died of CV causes. The median time from ED presentation until death was 72 days (IQR 20 - 167) and until death from CV causes 82 days (IQR 26 - 293). Patients who developed MACE had a higher 1-year all-cause mortality compared to patients who did not (46.5 vs. $28.7 \%$ resp., p-value 0.003 , Figure 7.2 ).

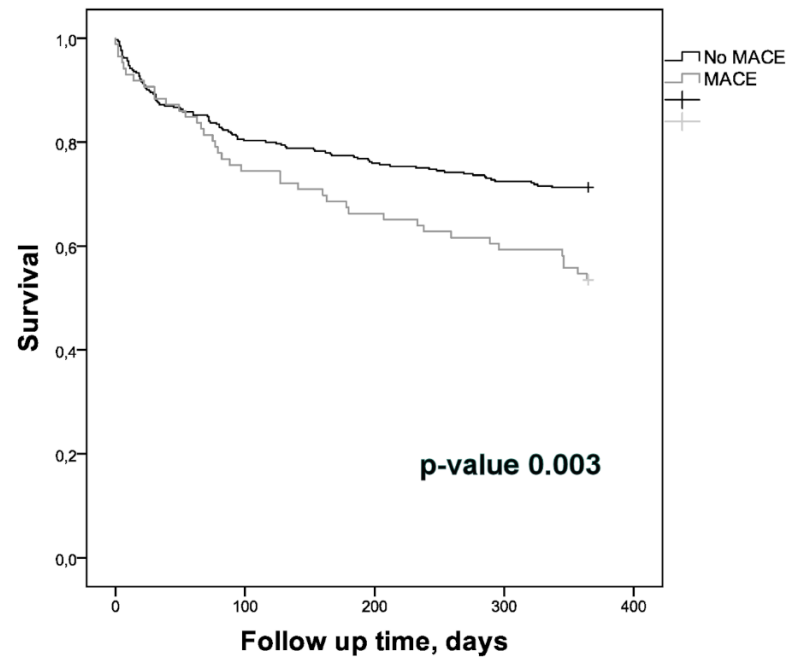

Figure 7.2 Survival curve of patients with and without MACE.

\section{Discussion}

In this prospective study, we demonstrated that older patients are at high risk (20.0\%) of developing MACE within 1 year after visiting the ED for non-cardiac complaints. The median time to MACE was 50 days with heart failure (54.6\%) and CVA/TIA (26.7\%) being the most prevalent presentations. Both hs-cTnT and NT-proBNP at the index visit were predictive of subsequent MACE (AUC of 0.74 for both), but only NT-proBNP proved to be an independent predictor of MACE on multivariable analysis correcting for other possible predictors of MACE. One-year mortality was high, since one third of our patients died within 1 year after their ED visit. Of these deaths, approximately $15 \%$ was certainly due to CV causes. In patients who developed MACE, 1-year mortality was higher than in those who did not (46.5 vs. $28.7 \%$, resp.). 
One out of five patients developed MACE within 1 year after their ED visit, which appears a very high number considering that these patients presented with noncardiac complaints. The reason for this high incidence of subsequent MACE is not known, but may be due to the fact that the majority of patients had substantial CV comorbidities and CV risk factors. Another explanation may be that approximately one third of our patients presented to the ED with an infection. Previously, patients with chronic inflammation or infections (including sepsis or pneumonia) were shown to be at high risk of developing CVD, and risks appeared even higher in septic patients with organ failure. ${ }^{3,7,21,22}$ The mechanism of interaction between infection/inflammation and CVD is complex and poorly understood, but is probably related to myocardial damage by proinflammatory cytokines and/or bacterial pathogens, coagulation disorders, induction of hypoxemia and ischaemia and/or progression of atherosclerosis possibly involving plaque rupture. ${ }^{23,24}$ Since many of the older patients presented with elevated biomarker values, it is possible that MACE was already present at the time of the ED visit, despite the fact that MACE was not diagnosed at that time. We however opinion that a combination of CV risk factors, a chronic inflammatory state due to ageing ${ }^{22}$ and/or chronic diseases ${ }^{25}$, and an acute medical problem (with or without an acute inflammatory state) probably contributed largely to the high subsequent occurrence of MACE in our patients.

To the best of our knowledge, no previous study examined the predictive ability of hscTnT and NT-proBNP for subsequent MACE in older patients who present to the ED with non-cardiac medical complaints. Apart from several cardiac diseases, hs-cTnT and NT-proBNP can be elevated due to non-cardiac medical conditions, including sepsis, renal failure and pulmonary embolisms ${ }^{19,26}$, which could consequently influence their predictive value. We, like others ${ }^{9-11,27-29}$, found that these biomarkers were predictive of MACE with a moderate discriminatory value (AUC of 0.74 for both). However, these other studies were performed in other clinical settings, e.g. in patients with acute coronary syndrome, stable heart failure, pneumoniae or in haemodialysis or noncardiac surgery patients. We further found that NT-proBNP was an independent predictor of MACE, while hs-cTnT was not. That NT-proBNP is the stronger independent predictor of MACE compared to hs-cTnT, was found in cardiac and haemodialysis patients as well. ${ }^{11,27}$ Several factors may explain this independent and compelling predictive power. Half of the MACEs were due to heart failure and approximately $25 \%$ of our MACE patients had a history of heart failure. In addition, non-cardiac medical conditions which could increase the risk of MACE (e.g. ageing, hyperthyroidism, renal failure, sepsis, shock and pulmonary embolism), may have caused elevated NT-proBNP levels ${ }^{19,29}$ and many of these conditions were present 
among the patients in our cohort. We conclude that NT-proBNP is a useful independent predictor of subsequent MACE in older non-cardiac ED patients, who have a high risk of developing MACE.

One third of our patients died within 1 year after their ED visit, which definitely reflects the vulnerability of this older population. Only $15 \%$ of these deaths were certainly due to CV causes which is relatively low. However, CV mortality is probably underestimated in our cohort since in most patients no post mortem exams were performed. Illustrative of this underestimation may be the finding that patients who developed MACE, more frequently died within 1 year $(46.5 \%)$ compared to patients who did not (28.7\%). Since the incidence of MACE and 1-year mortality in older ED patients is so high, we think it could be worthwhile to closely monitor high-risk patients after their ED visit in an attempt to improve their outcomes.

\section{Study strength, limitations and future perspectives}

The strength of our study is its prospective design in an older ED population presenting with non-cardiac complaints and the fact that we prospectively measured hs-cTnT and NT-proBNP regardless of the reason of ED visit. However, apart from the abovementioned limitation (regarding the probable underestimation of CV mortality), it is possible that CV comorbidities and classical CV risk factors were not registered completely. When not noted, we assumed these to be absent, which could have led to information bias. In addition, we cannot rule out that in some patients MACE was missed during the index visit, which could have affected our results. However, we carefully checked all the medical files for complaints indicative for MACE during the ED visit. Last, as physicians had to give priority to providing emergency care, not every possible candidate could be included in the study. We investigated possible selection bias by comparing the baseline characteristics and outcomes of the included with nonincluded patients and found no evidence for this. ${ }^{20}$

Since NT-proBNP is a strong independent marker for MACE in older patients who present with non-cardiac complaints, this biomarker is probably of additional value in identifying patients at high risk. In addition, studies investigating specific preventive therapies for MACE in high-risk non-cardiac older patients appear warranted.

\section{Conclusions}

Older patients who present to the ED with non-cardiac medical complaints are at high risk of developing MACE and dying within 1 year after their ED visit. Both hs-cTnT and 
NT-proBNP are predictors of subsequent MACE in this ED population, while only NTproBNP is an independent predictor of MACE. Prospective studies are needed to investigate whether specific or adjusted treatments are able to prevent MACE in these medical patients and if this subsequently improves their outcome. 


\section{References}

1. Aminzadeh F, Dalziel WB. Older adults in the emergency department: a systematic review of patterns of use, adverse outcomes, and effectiveness of interventions. Ann Emerg Med. 2002;39(3):238-47.

2. Samaras N, Chevalley T, Samaras D, Gold G. Older patients in the emergency department: a review. Ann Emerg Med. 2010;56(3):261-9.

3. Tralhão A, Póvoa P. Cardiovascular Events After Community-Acquired Pneumonia: A Global Perspective with Systematic Review and Meta-Analysis of Observational Studies. J Clin Med. 2020;9(2).

4. Tan SY, Sungar GW, Myers J, Sandri M, Froelicher V. A simplified clinical electrocardiogram score for the prediction of cardiovascular mortality. Clin Cardiol. 2009;32(2):82-6.

5. Bax L, Algra A, Mali WP, Edlinger M, Beutler JJ, van der Graaf Y. Renal function as a risk indicator for cardiovascular events in 3216 patients with manifest arterial disease. Atherosclerosis. 2008;200(1):184-90.

6. De Leeuw PW, Thijs L, Birkenhäger WH, Voyaki SM, Efstratopoulos AD, Fagard RH, et al. Prognostic significance of renal function in elderly patients with isolated systolic hypertension: results from the Syst-Eur trial. J Am Soc Nephrol. 2002;13(9):2213-22.

7. de Groot B, van den Berg S, Kessler J, Ansems A, Rijpsma D. Independent predictors of major adverse cardiovascular events in emergency department patients who are hospitalised with a suspected infection: a retrospective cohort study. BMJ Open. 2016;6(1):e009598.

8. Mokhtari A, Lindahl B, Smith JG, Holzmann MJ, Khoshnood A, Ekelund U. Diagnostic Accuracy of HighSensitivity Cardiac Troponin T at Presentation Combined With History and ECG for Ruling Out Major Adverse Cardiac Events. Ann Emerg Med. 2016;68(6):649-58.e3.

9. Aimo A, Januzzi JL, Jr., Vergaro G, Ripoli A, Latini R, Masson S, et al. High-sensitivity troponin T, NTproBNP and glomerular filtration rate: A multimarker strategy for risk stratification in chronic heart failure. Int J Cardiol. 2019;277:166-72.

10. Zhang S, Zhang HX, Lin RY, Zhang SM, Xu ZY. Predictive role of NT-pro BNP for adverse cardiac events in community-acquired pneumonia: A retrospective study. 2016;9:14411-7.

11. Onda T, Inoue K, Suwa S, Nishizaki Y, Kasai T, Kimura Y, et al. Reevaluation of cardiac risk scores and multiple biomarkers for the prediction of first major cardiovascular events and death in the drugeluting stent era. Int J Cardiol. 2016;219:180-5.

12. Zethelius B, Berglund L, Sundström J, Ingelsson E, Basu S, Larsson A, et al. Use of Multiple Biomarkers to Improve the Prediction of Death from Cardiovascular Causes. N Engl J Med. 2008;358(20):2107-16.

13. Park KC, Gaze DC, Collinson PO, Marber MS. Cardiac troponins: from myocardial infarction to chronic disease. Cardiovasc Res. 2017;113(14):1708-18.

14. Hall C. NT-ProBNP: the mechanism behind the marker. J Cardiac Fail. 2005;11(5 Suppl):S81-3.

15. Zelis N, Buijs J, de Leeuw PW, van Kuijk SMJ, Stassen PM. Study protocol for a multicentre prospective cohort study to identify predictors of adverse outcome in older medical emergency department patients (the Risk Stratification in the Emergency Department in Acutely Ill Older Patients (RISE UP) study). BMC Geriatr. 2019;19(1):65.

16. von Elm E, Altman DG, Egger M, Pocock SJ, Gøtzsche PC, Vandenbroucke JP. The Strengthening the Reporting of Observational Studies in Epidemiology (STROBE) statement: guidelines for reporting observational studies. J Clin Epidemiol. 2008;61(4):344-9.

17. Charlson ME, Pompei $\mathrm{P}$, Ales KL, MacKenzie CR. A new method of classifying prognostic comorbidity in longitudinal studies: development and validation. J Chronic Dis. 1987;40(5):373-83.

18. World Health O. International statistical classification of diseases and related health problems. 10th revision, Fifth edition, 2016 ed. Geneva: World Health Organization; 20152015.

19. Tsai SH, Lin YY, Chu SJ, Hsu CW, Cheng SM. Interpretation and use of natriuretic peptides in noncongestive heart failure settings. Yonsei Med J. 2010;51(2):151-63.

20. Zelis N, Buijs J, de Leeuw PW, van Kuijk SMJ, Stassen PM. A new simplified model for predicting 30day mortality in older medical emergency department patients: The rise up score. Eur J Intern Med. 2020;77:36-43. 
21. Bergh C, Fall K, Udumyan R, Sjöqvist H, Fröbert O, Montgomery S. Severe infections and subsequent delayed cardiovascular disease. Eur J Prev Cardiol. 2017;24(18):1958-66.

22. Ferrucci L, Fabbri E. Inflammageing: chronic inflammation in ageing, cardiovascular disease, and frailty. Nat Rev Cardiol. 2018;15(9):505-22.

23. Mankowski RT, Yende S, Angus DC. Long-term impact of sepsis on cardiovascular health. Intensive Care Med. 2019;45(1):78-81.

24. Corrales-Medina VF, Musher DM, Shachkina S, Chirinos JA. Acute pneumonia and the cardiovascular system. Lancet (London, England). 2013;381(9865):496-505.

25. Furman D, Campisi J, Verdin E, Carrera-Bastos P, Targ S, Franceschi C, et al. Chronic inflammation in the etiology of disease across the life span. Nat Med. 2019;25(12):1822-32.

26. Korff S, Katus HA, Giannitsis E. Differential diagnosis of elevated troponins. Heart. 2006;92(7):987-93.

27. Satyan S, Light RP, Agarwal R. Relationships of N-terminal pro-B-natriuretic peptide and cardiac troponin $\mathrm{T}$ to left ventricular mass and function and mortality in asymptomatic hemodialysis patients. Am J Kidney Dis. 2007;50(6):1009-19.

28. Leistner DM, Klotsche J, Pieper L, Palm S, Stalla GK, Lehnert H, et al. Prognostic value of NT-pro-BNP and hs-CRP for risk stratification in primary care: results from the population-based DETECT study. Clin Res Cardiol. 2013;102(4):259-68.

29. Zhang L-J, Li N, Li Y, Zeng X-T, Liu M-Y. Cardiac Biomarkers Predicting MACE in Patients Undergoing Noncardiac Surgery: A Meta-Analysis. Front Physiol. 2019;9:1923. 


\section{Supplemental data}

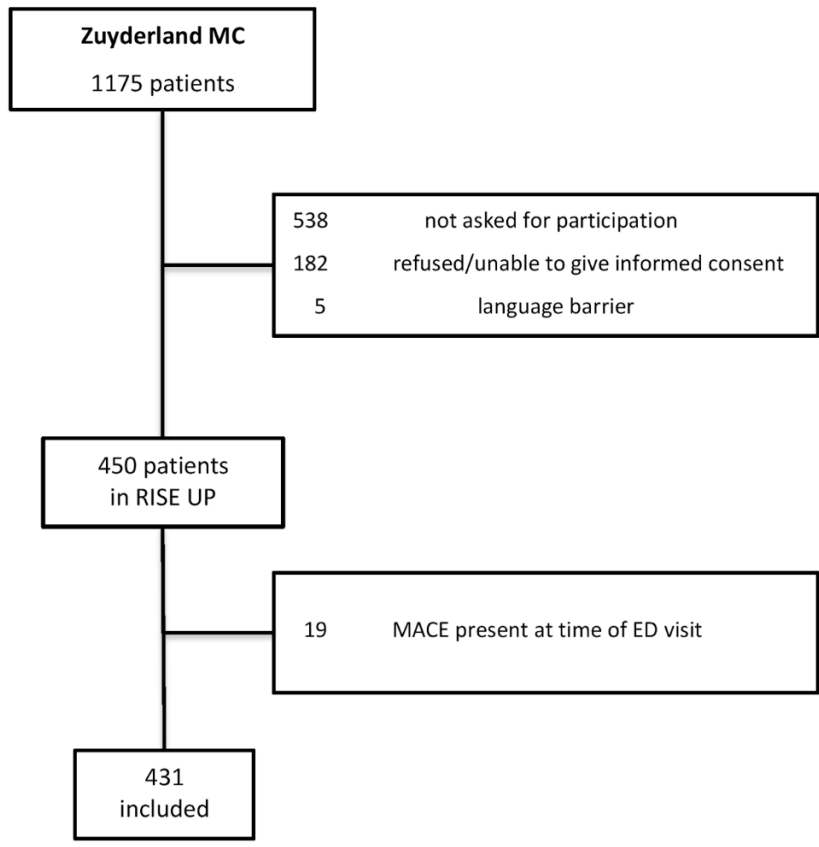

Figure S7.1 Flowchart of patient selection.

Table S7.1 Baseline characteristics of the included and non-included patients. ${ }^{\text {a }}$

\begin{tabular}{lcc}
\hline Characteristic & $\begin{array}{c}\text { Included } \\
\text { (n=450) }\end{array}$ & $\begin{array}{c}\text { Non-included } \\
\text { (n=200) }\end{array}$ \\
\hline Median age (IQR), years & $79(73-85)$ & $78(72-85)$ \\
Male sex, $\mathrm{n}(\%)$ & $234(52.0)$ & $100(50.0)$ \\
Living in nursing- or care home, $\mathrm{n}(\%)$ & $39(8.7)$ & $23(11.7)$ \\
Median CCl score (IQR) & $2(1-3)$ & $2(1-3)$ \\
Manchester triage category, $\mathrm{n}(\%)$ & $56(12.5)$ & $38(19.2)$ \\
$\quad$ Orange & $254(56.8)$ & $108(54.5)$ \\
Yellow & $133(29.8)$ & $51(25.8)$ \\
$\quad$ Green & & $153(76.5)$ \\
Treatment, $\mathrm{n}(\%)$ & $326(72.4)$ & $171(85.5)$ \\
$\quad$ Internal medicine & $366(81.3)$ & $30(15.0)$ \\
$\quad$ Admission & $51(11.3)$ & \\
30-day mortality, $\mathrm{n}(\%)$ & & \\
\hline
\end{tabular}

$\mathrm{CCl}$, Charlson comorbidy Index score; IQR, interquartile range. Numbers are values (\%) unless stated otherwise. ${ }^{a}$ In total, 450 patients were included in the derivation cohort during the prospective study period in Zuyderland MC (included patients). The non-included patients represent a sample of 200 patients who were not included during the prospective study period for reasons other than refusal; ${ }^{b}$ There were no significant differences in baseline characteristics between patient who were included and patients who were not included. 


\section{Chapter 8}

RISE UP score: predicting prognosis in patients with COVID-19 in the emergency department

P.M.E.L. van Dam, N. Zelis, P.M. Stassen, D.J.L. van Twist, P.W. de Leeuw,

S.M.J. van Kuijk, J. Buijs 


\section{Abstract}

\section{Background}

COVID-19 presents an important and urgent threat to global health. To mitigate the burden on the healthcare system, information on the prognosis of the disease is needed. Prediction models may help to estimate the risk of poor outcome. The recently developed RISE UP score is an easy to apply model with very good discriminatory value with respect to short-term mortality in older emergency department (ED) patients. It consists of six items: age, $\geq 2$ abnormal vital signs, albumin, blood urea nitrogen (BUN), lactate dehydrogenase (LDH) and bilirubin. As many hospitalized COVID-19 patients are old, we hypothesized that the RISE UP score could have discriminatory value with regard to 30-day mortality in ED patients with COVID-19 as well.

\section{Materials and methods}

Retrospective analysis of ED patients with COVID-19 in one of the largest teaching hospitals in the Netherlands. Primary outcome was 30-day mortality, secondary outcome was a composite of 30-day mortality and admission to the intensive care unit (ICU).

\section{Results}

The study sample consisted of 642 consecutive ED patients diagnosed with COVID-19 in the period from March $3^{\text {rd }}$ until May $25^{\text {th }} 2020$. Within 30 days after presentation, 167 patients (26.0\%) died and 102 patients (15.9\%) were admitted to ICU. The RISE UP score showed good discriminatory value with respect to 30-day mortality (AUC 0.77, $95 \% \mathrm{Cl}$ 0.73-0.81) and to the composite outcome of 30-day mortality and/or ICU admission (AUC $0.72,95 \% \mathrm{Cl} 0.68-0.76$ ). Patients with a RISE UP score below $10 \%$ had favorable outcome and patients with a RISE UP score above $30 \%$ had a high chance of adverse outcome.

\section{Conclusion}

The RISE UP score is an accurate and easy to apply prediction model for 30-day mortality and ICU admission in ED patients with COVID-19. This model can be used to recognize patients at low or high risk of short-term adverse outcome and may assist in guiding clinical decision-making and allocating healthcare resources. 


\section{Background}

The COVID-19 pandemic has affected millions of people, and has put enormous strains on healthcare resources. ${ }^{1-4}$ To mitigate the burden on the healthcare system, information on the prognosis of the patients is needed. Although most patients with COVID-19 develop only mild symptoms, some develop severe and potentially fatal complications. ${ }^{1,2,5,6}$.

Prediction models could help to estimate the risk of a poor or good outcome and may therefore assist in triaging patients when allocating healthcare resources. Many risk prediction models have been developed for patients with COVID-19, most of which still need rigorous external validation efforts to assess their added value to clinical practice. However, our group developed and validated the RISE UP score (Risk Stratification in the Emergency Department in Acutely III Older Patients), an accurate and easy to use model, predicting mortality in medical patients older than 65 years in the emergency department (ED). ${ }^{7}$ The model had very good discriminatory value with an area under the receiver operating characteristic curve (AUC) of 0.83 .

We hypothesized that the RISE UP score may also be valuable in predicting prognosis in patients with COVID-19, as this disease predominantly affects the same age group. The aim of the present retrospective study was to evaluate the discriminatory value of the RISE UP score with regard to poor outcome (mortality and/or admission to the intensive care unit (ICU)) in ED patients with COVID-19.

\section{Materials and methods}

This study has been reported in accordance with the Transparent reporting of a multivariable prediction model for individual prognosis or diagnosis (TRIPOD): The TRIPOD statement. ${ }^{8}$

\section{Study design and setting}

We performed a retrospective analysis at the two EDs of the Zuyderland Medical Centre (MC), location Heerlen and Sittard-Geleen. This is one of the largest teaching hospitals in the Netherlands providing secondary care to a total of 55,000 patients during ED visits on a yearly basis. This study was approved by the medical ethics 
committee of the Zuyderland MC (METCZ20200136). Informed consent was obtained retrospectively by an opt-out method by writing a letter to the included patients.

\section{Study sample}

The study sample consisted of consecutive adult (18 years or older) medical ED patients diagnosed with COVID-19 in the period from March $3^{\text {rd }}$ until May $25^{\text {th }} 2020$. Patients were included if they met the following criteria: 1) admission to the hospital with symptoms suggestive of COVID-19 (i.e. coughing, common cold, sore throat, dyspnea, acute diarrhea, vomiting, fever, or an unexpectedly discovered oxygen saturation below 92\%); and 2) positive result of the polymerase chain reaction (PCR) for SARS-CoV-2 in respiratory specimens; or 3) (very) high suspicion of COVID-19 according to the computed tomography (CT) scan of the thorax (CO-RADS 4 or CORADS 5, see Table 8.1). ${ }^{9}$ We excluded patients who revisited the ED after an earlier ED presentation during the study period.

Table 8.1 Overview of CO-RADS categories for suspicion of pulmonary involvement of COVID-19.

\begin{tabular}{lll}
\hline & Level of suspicion & Summary \\
\hline CO-RADS 1 & Very low & Normal or non-infectious \\
CO-RADS 2 & Low & Typical for other infection but not COVID-19 \\
CO-RADS 3 & Equivocal / unsure & Features compatible with COVID-19, but also other diseases \\
CO-RADS 4 & High & Suspicious for COVID-19 \\
CO-RADS 5 & Very high & Typical for COVID-19 \\
\hline
\end{tabular}

CO-RADS, COVID-19 Reporting And Data System.

\section{Data collection}

From electronic medical records, we collected data on age, sex, and information regarding comorbidity according to the Charlson Comorbidity Index $(\mathrm{CCl}) .{ }^{10}$ The following vital signs were retrieved: heart rate (HR), mean arterial blood pressure (MAP), respiratory rate (RR), oxygen saturation, temperature, and Glasgow Coma Scale (GCS). When the RR or GCS were missing, we used paCO2 and descriptions in the medical records to deduce these values, similar to other studies. ${ }^{71,12}$ In addition, we collected routinely assessed laboratory tests: blood urea nitrogen (BUN), lactate dehydrogenase (LDH), bilirubin, albumin, lymphocytes, and C-reactive protein (CRP). We retrieved all items of the RISE UP score. The original formula of the RISE UP score to calculate an individual's probability of 30-day mortality is: $\mathrm{P}(30$-day mortality) $=1$ / $\left(1+\exp \left(-\left(-3.908+0.050 *\right.\right.\right.$ Age $+1.115^{*} \geq 2$ Abnormal Vital Signs (yes=1, no=0) - 
$0.112 *$ Albumin (in $\mathrm{g} / \mathrm{L})+0.284 *(\mathrm{BUN}$ (in $\mathrm{mmol} / \mathrm{L}) / 5)+0.120 *(\mathrm{LDH}$ (in U/L)/100) + $0.875^{*}$ Bilirubin $>20 \mu \mathrm{mol} / \mathrm{L}($ yes $\left.\left.=1, \mathrm{no}=0)\right)\right)^{7}$

Furthermore, we collected the results of the reverse-transcriptase PCR for SARS-CoV-2 in respiratory specimens and the results of the CT scan of the thorax. Finally, we retrieved data on length of hospital stay, admission to the intensive care unit (ICU) and mortality. Data on mortality were verified using medical records, which are connected to the municipal administration office.

\section{Outcomes}

The primary outcome was all-cause mortality within 30 days after ED presentation. The secondary outcome was a composite of mortality within 30 days after ED presentation and admission to the ICU. In our hospital, the main reason for admitting patients to the ICU was mechanical ventilator support.

\section{Statistical analysis}

Baseline characteristics were analyzed using descriptive statistics on the observed data. For each included patient, we calculated their individual probability of 30-day mortality and of the composite endpoint of 30-day mortality and/or admission to ICU using the initial RISE UP score formula. In case the probability could not be computed in over $5 \%$ of patients due to missing values, data would be imputed using multiple imputation, as per our study protocol. Predictive performance of the RISE UP score in this external validation was quantified as discriminative ability (i.e., the ability of the model to separate those who will experience the event from those who will not) and model calibration. We calculated the AUC of the receiver operating characteristic (ROC) curve to quantify the discriminative ability. An AUC of 0.5 would correspond to no more discriminative value than the toss of a coin, whereas an AUC of 1.0 would mean perfect separation. Calibration of the RISE UP score was assessed by visually inspecting the calibration plot that shows the agreement between predicted and observed probabilities for subgroups of patients based on similar predicted risk. In case of poor calibration (i.e., the intercept of the plot is not equal to 0 and/or the slope of the plot is not equal to 1), the model was recalibrated using logistic regression analysis with the linear predictor of the RISE UP score, the linear sum of the regression coefficient multiplied by their respective predictor values, as the only independent variable.

Finally, we aimed to identify cut off values of the RISE UP score that will support clinical decision-making, by identifying patients with low and high risk of an adverse outcome (low risk and high risk group). To achieve this, we calculated sensitivity, 
specificity, positive and negative predictive values from multiple cut off values of the RISE UP score.

All data were analyzed using IBM SPPS Statistics for Windows, IBM Corporation, Armonk N.Y., USA, version 25.0.

\section{Results}

\section{Study sample}

During the study period, 642 consecutive ED patients diagnosed with COVID-19 met the inclusion criteria and were included (Table 8.2). The median age of patients was 72 years (IQR 62-80), and $69.2 \%$ were 65 years or older. Most patients (63.4\%) were male. More than half of the patients $(59.8 \%)$ had two or more abnormal vital signs. The laboratory results showed elevated LDH (median $350 \mathrm{U} / \mathrm{L}$ ). The median length of hospital stay was 6 days (IQR 3-12).

In our sample, 102 patients (15.9\%) were admitted to ICU and 167 patients died, yielding a 30 -day mortality of $26.0 \%$. The median number of days until death was 8 (IQR 4-15, Figure 8.1).

\section{RISE UP score}

The RISE UP score could not be calculated in 21 patients (3.2\%) because of missing values (laboratory tests). As this was below our $5 \%$ threshold, no imputation step was performed. For 621 patients the RISE UP score yielded an AUC of $0.77(95 \% \mathrm{Cl}$ : 0.73-0.81) with respect to the 30-day mortality, and an AUC of 0.72 (95\% Cl 0.68-0.76) with respect to the composite endpoint of 30-day mortality and/or admission to the ICU.

The initial calibration plot showed average underestimation of 30-day mortality by the model and a slope of $>1$ (Figure 8.2). This could at least in part be due to the fact that in the derivation and validation cohort of the RISE UP score we observed a 30-day mortality of $10.9 \%$ and $13.3 \%$, respectively. Therefore, we performed recalibration of the model. The formula was adjusted by changing the intercept to -2.083 and multiplying the linear sum of regression coefficients multiplied by their respective predictor values by 0.795 . The adjusted formula for the RISE UP score in patients with COVID-19 was: $\mathrm{P}(30$-day mortality $)=1 /(1+\exp (-(-2.083+0.795 *(0.050 *$ Age + $1.115^{*} \geq 2$ Abnormal Vital Signs (yes $=1, \mathrm{no}=0$ ) $-0.112 *$ Albumin (in $\mathrm{g} / \mathrm{L}$ ) $+0.284 *$ (BUN (in $\mathrm{mmol} / \mathrm{L}) / 5)+0.120^{*}(\mathrm{LDH}$ (in $\left.\mathrm{U} / \mathrm{L}) / 100\right)+0.875^{*}$ Bilirubin $>20 \mu \mathrm{mol} / \mathrm{L}$ (yes $=1$, no=0)))). 
Table $8.2 \quad$ Baseline characteristics of study population.

\begin{tabular}{|c|c|c|}
\hline & Reference values & Study sample $(n=642)$ \\
\hline \multicolumn{3}{|l|}{ Age } \\
\hline Age, median (IQR), years & & $72(62-80)$ \\
\hline 65 years or older, $n \%$ & & $444(69.2)$ \\
\hline Male, $\mathrm{n} \%$ & & $407(63.4)$ \\
\hline CCI score, median (IQR) & & $1(0-3)$ \\
\hline \multicolumn{3}{|l|}{ Abnormal vital signs, n\% } \\
\hline Tachycardia (HR >90 bpm) & & $330(51.4)$ \\
\hline Hypotension (MAP < 70 mmHg) & & $13(2.0)$ \\
\hline Tachypnea (RR >20/min) & & $367(57.2)$ \\
\hline O2 saturation $<95 \%$ & & $325(50.6)$ \\
\hline Hypo- or hyperthermia $\left(<36\right.$ or $\left.>38^{\circ} \mathrm{C}\right)$ & & $332(51.7)$ \\
\hline GCS $<15$ & & $96(15.0)$ \\
\hline$\geq 2$ abnormal vital signs ${ }^{a}$ & & $384(59.8)$ \\
\hline \multicolumn{3}{|l|}{ Laboratory results } \\
\hline Lymphocytes, median (IQR), x 109\% /L & $1.1-4.0$ & $0.85(0.59-1.22)$ \\
\hline BUN, median (IQR), mmol/L & $3.5-7.5$ & $6.9(5.0-10.0)$ \\
\hline Bilirubin, median (IQR), $\mu \mathrm{mol} / \mathrm{L}$ & $<21$ & $9.0(7.0-13.0)$ \\
\hline LDH, median (IQR), U/L & $<248$ & $350(269-462)$ \\
\hline Albumin, mean (SD), g/L & $35-50$ & $37.4(4.3)$ \\
\hline CRP, median (IQR), mg/L & $<10$ & $77(36-130)$ \\
\hline Positive PCR for SARS-CoV-2, n\% & & $553(86.1)$ \\
\hline CO-RADS 4 or CO-RADS 5 on CT thorax, n\% & & $366(57.0)$ \\
\hline Intensive care unit (ICU) admission, n\% & & $102(15.9)$ \\
\hline Length of hospital stay, median (IQR), days & & $6(3-12)$ \\
\hline \multicolumn{3}{|l|}{ Outcome } \\
\hline Mortality within 30 days, n\% & & $167(26.0)$ \\
\hline Composite endpoint (mortality and/or ICU), n\% & & $226(35.2)$ \\
\hline Number of days until death, median (IQR) & & $8(4-15)$ \\
\hline
\end{tabular}

BUN, blood urea nitrogen; $\mathrm{CCl}$, Charlson comorbidity index; CO-RADS, COVID-19 Reporting And Data System; CRP, C-reactive protein; GCS, Glasgow coma scale; HR, heart rate; IQR, interquartile range; $L D H$, lactate dehydrogenase; MAP, mean arterial pressure; PCR, polymerase chain reaction; RR, respiratory rate; $\mathrm{SD}$, standard deviation. ${ }^{\mathrm{a}} \geq 2$ of following abnormal vital signs: $\mathrm{HR}>90 \mathrm{bpm}, \mathrm{MAP}<70 \mathrm{mmHg}, \mathrm{RR}>20 / \mathrm{min}$, O2 saturation $<95 \%$ and GCS $<15$.

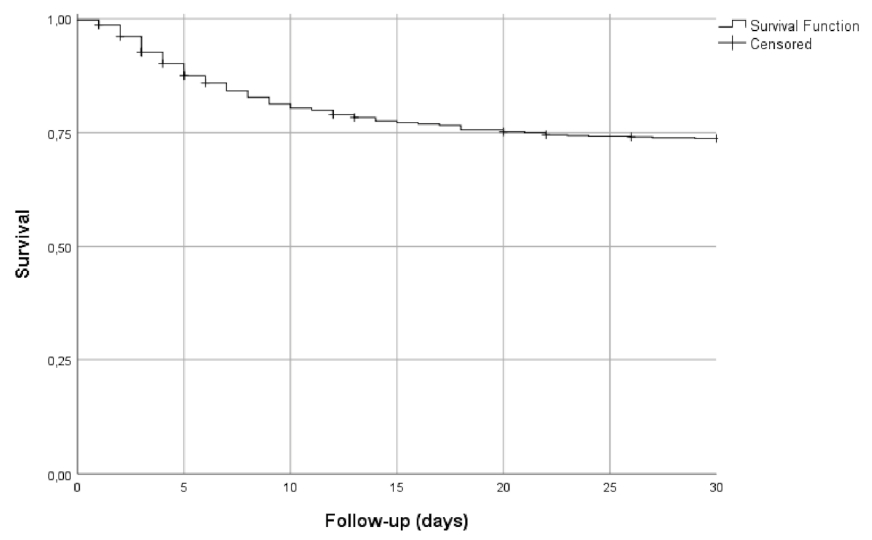

Figure 8.1 Kaplan Meier surviving curve. 

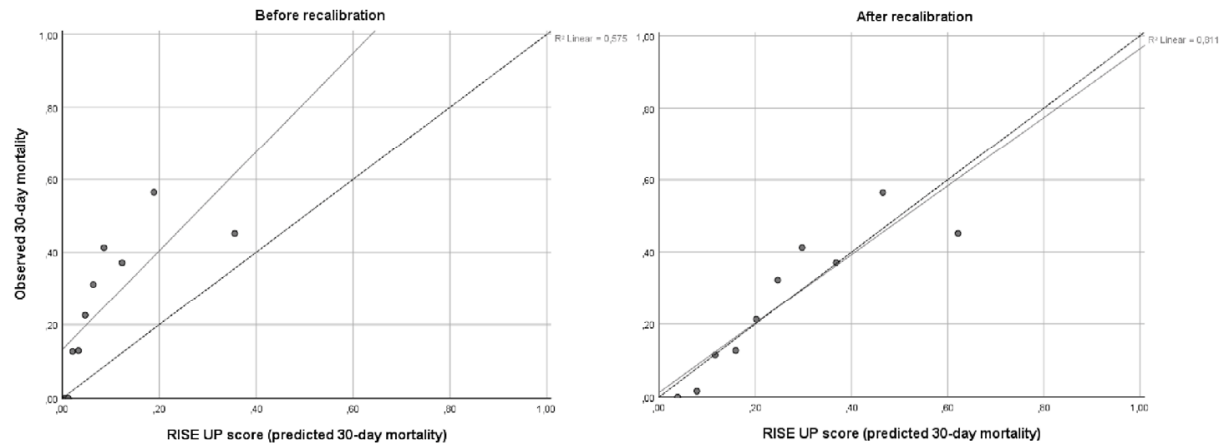

Figure 8.2 Calibration plots before and after recalibration.

\section{Determination of clinical cut off values}

The individual RISE UP scores were stratified according to their probability to predict 30-day mortality. Patients with a RISE UP score $<10 \%$ were considered to be at (very) low risk of mortality (Table 8.3 ). In this subgroup of 121 patients (19.5\%), there were no deaths and only six were admitted to ICU, yielding a sensitivity of $100 \%(95 \% \mathrm{Cl}$ 97.7-100) and a negative predictive value of $100 \%$. A RISE UP score $<5 \%$ also yielded a sensitivity and negative predictive value of $100 \%$, however, this subgroup of patients consisted of 41 patients only (6.6\%).

Patients with a RISE UP score $>30 \%$ were at high risk of a poor outcome (Table 8.3 ). In this subgroup of 221 patients (35.6\%), we found a mortality of approximately $50 \%$ and $15 \%$ ICU admission.

Table 8.3 Cut off values of the RISE UP score.

\begin{tabular}{|c|c|c|c|c|c|c|c|}
\hline $\begin{array}{l}\text { Cut off } \\
\text { value (\%) }\end{array}$ & $\begin{array}{c}\text { Patients } \\
(\mathrm{n} \%)^{\mathrm{a}}\end{array}$ & $\begin{array}{l}\text { Deaths } \\
(n \%)^{b}\end{array}$ & $\begin{array}{l}\text { Admissions } \\
\text { to ICU }(\mathrm{n} \%)^{\mathrm{b}}\end{array}$ & $\begin{array}{l}\text { Sensitivity } \\
(\%, 95 \% \mathrm{Cl})^{c}\end{array}$ & $\begin{array}{l}\text { Specificity } \\
(\%, 95 \% \mathrm{Cl})^{\mathrm{c}}\end{array}$ & $\begin{array}{c}\text { PPV } \\
(\%, 95 \% \mathrm{Cl})^{\mathrm{c}}\end{array}$ & $\begin{array}{c}\text { NPV } \\
(\%, 95 \% \mathrm{Cl})^{\mathrm{c}}\end{array}$ \\
\hline$<10$ & $\begin{array}{c}121 \\
(19.5)\end{array}$ & $\begin{array}{c}0 \\
(0)\end{array}$ & $\begin{array}{c}6 \\
(5.0)\end{array}$ & $\begin{array}{c}100 \\
(97.7-100)\end{array}$ & $\begin{array}{c}26.3 \\
(22.3-30.6)\end{array}$ & $\begin{array}{c}32.2 \\
(31.1-33.5)\end{array}$ & 100 \\
\hline$>30$ & $\begin{array}{c}221 \\
(35.6)\end{array}$ & $\begin{array}{c}103 \\
(46.6)\end{array}$ & $\begin{array}{c}42 \\
(19.0)\end{array}$ & $\begin{array}{c}64.0 \\
(56.1-71.4)\end{array}$ & $\begin{array}{c}74.3 \\
(70.1-78.3)\end{array}$ & $\begin{array}{c}47.4 \\
(41.9-51.6)\end{array}$ & $\begin{array}{c}85.5 \\
(82.6-87.4)\end{array}$ \\
\hline
\end{tabular}

$\mathrm{Cl}$, confidence interval; ICU, intensive care unit; $\mathrm{n}$, number of cases; NPV, negative predictive value; PPV, positive predictive value; RISE UP, Risk stratification in the emergency department in acutely ill older patients. ${ }^{a}$ The total number of patients described in this table is 621 , because of missing RISE UP scores in 21 patients; ${ }^{b}$ The percentages of deaths and ICU admissions are related to the amount of patients above or

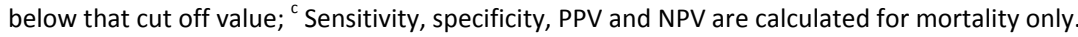




\section{Discussion}

In this retrospective study in two EDs in the Netherlands, we found that the RISE UP score showedm $n$ good discriminatory value with respect to 30-day all-cause mortality and/or admission to ICU in ED patients with COVID-19. The model yielded an AUC of 0.77 (95\% Cl 0.73-0.81) for 30-day mortality and an AUC of 0.72 (95\% Cl 0.68-0.76) for a composite of 30-day mortality and/or admission to ICU. After performing recalibration the RISE UP score was well calibrated. We found that patients with a RISE UP score $<10 \%$ had favorable outcomes and that patients with a RISE UP score $>30 \%$ were at high risk of adverse outcomes.

Using the RISE UP score, the probability of both favorable and poor outcomes can be predicted in the first two hours of the ED visit. This is important because this may guide clinical decision-making, especially during the current COVID-19 pandemic with healthcare system facing problems with clinical healthcare facilities (i.e. shortage of ICU beds) and the development of out-of-hospital low care facilities (i.e. corona hotels). The finding that the RISE UP score may be useful to guide clinical decisionmaking is important, because a clinical algorithm like this score is essential for allocation of healthcare resources. The RISE UP score is applicable for both sides of the clinical spectrum. In our cohort, 121 patients (19.5\%) had a RISE UP score $<10 \%$, and had very low risk of short-term mortality or ICU admission. In these patients, the clinician can choose safely to refer the patient to outpatient treatment or to discharge at an early stage. On the other hand, the 74 patients (11.9\%) in our cohort with a RISE UP score $>30 \%$ had a high risk of adverse outcome. This may support the decision to transfer these patients to ICU at an early stage. Furthermore, in patients with high RISE UP scores, preexisting multiple comorbidities and poor clinical performance, the model might support the decision to choose supportive care only.

The RISE UP score was recently developed to predict 30-day all-cause mortality in older medical ED patients. ${ }^{7}$ The score consists of six easily and readily available items during the ED visit. The RISE UP score can be easily implemented in routine practice with an online calculator (https://jscalc.io/calc/9Jmqwo1QoVOBtdBB). The model has face validity as it includes items that are markers of severe disease, such as sepsis, renal failure, liver failure, or shock. Because of this, the RISE UP score reflects the severity of illness in ED patients. Therefore, it is not surprising that the model has good discriminatory value for poor outcome in ED patients with COVID-19, since our sample largely consisted of older medical patients (69.2\% were 65 years or older). Other studies have also shown higher mortality in older patients with COVID-19. ${ }^{13-16}$ 
We state that application of the RISE UP score easily identifies patients with COVID-19 who are at high and at low risk of dying within the first 30 days after presentation to the ED.

The original research article concerning the RISE UP score showed a better predictive performance compared to our current study (AUC 0.83 versus 0.77 ). ${ }^{7}$ The difference can be explained in several ways. First, 30 -day mortality was around $11 \%$ in the original cohort, while in our cohort it was $26.0 \%$. This can be explained because in this study, we only included ED patients who were admitted to the hospital, whereas patients that were discharged from the ED were excluded. Furthermore, COVID-19 can have a serious course in admitted patients. Therefore, the probability of a poor outcome was higher in our cohort. Secondly, the levels of LDH were often elevated in patients with COVID-19 ( $81.1 \%$ were higher than the reference value). Since LDH is one of the items in the RISE UP score, this may affect the discriminatory value. Third, in our clinical experience, some patients with COVID-19 show a rapid deterioration of their clinical condition, while there were few objective symptoms in the initial assessment. This phenomenon may play a role as well, since the RISE UP score includes the initial assessment only.

The RISE UP score still has good discriminatory value in a population that is different from the population in which it was designed. This can be explained because the model accurately reflects the severity of illness in ED patients. The severity is determined by both the severity of the disease, and preexisting comorbidities. In COVID-19, the prognosis is also determined by the disease itself and patient related factors (i.e. age and comorbidities). ${ }^{1,5}$

In a recent systematic review, a total of 16 prognostic models for predicting mortality, progression to severe disease or length of hospital stay in patients with COVID-19 were analyzed. ${ }^{17}$ The AUC estimates ranged from 0.85 to 0.99 . However, most proposed models were not clearly described and therefore it is unclear whether they are feasible in other settings. Furthermore, most scores were not externally validated. The authors of the systematic review do not recommend the use of any of these models in current practice. The RISE UP score is an accurate and externally validated prediction model with good discriminatory value in unselected older medical patients. We state that the RISE UP score can be used in current clinical practice.

Our study has several limitations. First, our study was performed in the two EDs of one medical centre which may limit generalizability of the results. However, our study has a relatively large cohort of patients with COVID-19 in one of the most heavily affected areas of the Netherlands. Second, the number of ICU admissions in our study 
was relatively low $(15.9 \%)$, which may result from decisions to initiate conservative care in patients with preexisting frailty or severe comorbidity. These decisions may be different in other countries, which made us decide to study ICU admissions as a composite outcome only.

The RISE UP score can be readily implemented in routine practice at the ED. It is easy to use and not time-consuming to fill in. Our current study shows that the discriminatory value in ED patients with COVID-19 is good and that the RISE UP score is therefore also applicable in these patients.

\section{Conclusion}

In conclusion, the RISE UP score is an accurate and easily available prediction model for 30-day mortality and ICU admission in ED patients with COVID-19. The score is useful to identify patients at low and high risk for clinical complications. So this model may contribute to fast recognition of patients who are at low or high risk for short term adverse outcome, and guide clinical decision-making and allocating healthcare resources in this severe pandemic, dealing with scarcity of clinical facilities and materials. 


\section{References}

1. Chen N, Zhou M, Dong X, et al. Epidemiological and clinical characteristics of 99 cases of 2019 novel coronavirus pneumonia in Wuhan, China: a descriptive study. Lancet 2020;395:507-513.

2. Sohrabi C, Alsafi Z, O'Neill N, et al. World Health Organization declares global emergency: A review of the 2019 novel coronavirus (COVID-19). Int J Surg 2020;76:71-76.

3. World Health Organization. Coronavirus disease (COVID-19): situation report - 134, https://www.who.int/docs/default-source/coronaviruse/situation-reports/20200602-covid-19-sitrep134.pdf?sfvrsn=cc95e5d5_2 (2020).

4. COVID-19 Coronavirus pandemic, https://www.worldometers.info/coronavirus/?utm_campaign= homeAdvegas1? (2020).

5. Guan WJ, Ni ZY, Hu Y, et al. Clinical Characteristics of Coronavirus Disease 2019 in China. N Engl J Med 2020;382:1708-1720.

6. Bruggemann $\mathrm{R}$, Gietema $\mathrm{H}$, Jallah $\mathrm{B}$, et al. Arterial and venous thromboembolic disease in a patient with COVID-19: A case report. Thromb Res 2020;191:153-155.

7. Zelis N, Buijs J, de Leeuw PW, et al. A new simplified model for predicting 30-day mortality in older medical emergency department patients: The rise up score. Eur J Intern Med 2020;77:36-43.

8. Collins GS, Reitsma JB, Altman DG, et al. Transparent reporting of a multivariable prediction model for individual prognosis or diagnosis (TRIPOD): the TRIPOD statement. BMJ (Clinical research ed) 2015; 350: g7594.

9. Prokop $\mathrm{M}$, van Everdingen W, van Rees Vellinga $\mathrm{T}$, et al. CO-RADS - A categorical CT assessment scheme for patients with suspected COVID-19: definition and evaluation. Radiology 2020:201473.

10. Charlson ME, Pompei $\mathrm{P}$, Ales $\mathrm{KL}$, et al. A new method of classifying prognostic comorbidity in longitudinal studies: development and validation. J Chronic Dis 1987;40:373-383..

11. Hilderink MJ, Roest AA, Hermans $M$, et al. Predictive accuracy and feasibility of risk stratification scores for 28-day mortality of patients with sepsis in an emergency department. Eur J Emerg Med 2015;22:331-337.

12. Howell MD, Donnino MW, Talmor D, et al. Performance of severity of illness scoring systems in emergency department patients with infection. Acad Emerg Med 2007;14:709-714.

13. Wang L, He W, Yu X, et al. Coronavirus disease 2019 in elderly patients: Characteristics and prognostic factors based on 4-week follow-up. J Infect 2020;80:639-645.

14. Shahid Z, Kalayanamitra R, McClafferty B, et al. COVID-19 and Older Adults: What We Know. J Am Geriatr Soc 2020;68:926-929.

15. Feng Y, Ling Y, Bai T, et al. COVID-19 with Different Severities: A Multicenter Study of Clinical Features. Am J Respir Crit Care Med 2020;201:1380-1388.

16. Zhou F, Yu T, Du R, et al. Clinical course and risk factors for mortality of adult inpatients with COVID19 in Wuhan, China: a retrospective cohort study. Lancet 2020;395:1054-1062.

17. Wynants L, Van Calster B, Bonten MMJ, et al. Prediction models for diagnosis and prognosis of covid19 infection: systematic review and critical appraisal. BMJ 2020;369:m1328. 


\section{Chapter 9}

Summary and general discussion 



\section{Summary and general discussion}

The number of emergency department (ED) visits by older people ( $\geq 65$ years) is rising and will remain to increase in the next decades. After an ED visit, older patients are at high risk of adverse outcomes, including mortality. Identification of such high risk patients during an ED visit is crucial in order to personalise and improve medical care. Risk stratification scores that accurately predict adverse outcome in older ED patients were however lacking and most of the scores available use items that are difficult to retrieve during the short ED stay. The aim of this thesis was to find accurate and easy tools to identify patients at risk of adverse outcomes. To this end, we tried to detect early predictors of adverse outcomes in older medical ED patients and to develop an easily applicable prediction model for short-term mortality.

To meet these goals, a prospective multicentre observational cohort study, the RIsk Stratification in the Emergency department in acUtely ill older Patients (RISE UP) study was conducted at the EDs in Zuyderland Medical Centre (MC) and Maastricht University Medical Centre+ (MUMC+), which provided the data for this thesis. Older patients ( $\geq 65$ years) were enrolled in this study when they visited the ED for internists or gastroenterologists from July 2016 until February 2017. An overview of the study design, objectives and procedures was presented in Chapter 2.

The main findings of this thesis are summarised and discussed below.

\section{Early predictors of adverse outcome}

The first aim of this thesis was to identify early predictors of adverse outcomes in older ED patients. For this purpose, the discriminatory value of the clinical intuition of patients, nurses and physicians and the concerns of patients and caregivers regarding the medical condition or care, were investigated. In addition, the discriminatory value of five relevant biomarkers were examined.

\section{Clinical intuition of patients, nurses and physicians}

In chapter 3 the discriminatory value of clinical intuition for 30-day mortality and other adverse outcomes (defined as a secondary composite endpoint of intensive- or medium care (ICU/MCU) admission, prolonged length of hospital stay (LOS), loss of independent living and unplanned readmission within 30 days after discharge) was described. Clinical intuition was defined as the disease perception and self-rated health of patients and caregivers and the clinical impression of nurses and physicians. 
Patients (or caregivers), nurses and physicians filled in a questionnaire, which contained several questions about the disease perception, self-rated health and clinical impression, including the surprise question (SQ). The questionnaires were filled in individually at the beginning of the ED visit, before history taking, physical examination and without knowledge of any diagnostic results. This first clinical impression was used because major decisions about prioritisation of diagnostics and treatments have to be made at this stage of the ED visit. The questions were scored using a Likert scale from 1 to 5 and were further referred to as scores. Patients, nurses and physicians filled in three identical scores: the severity of illness score (i.e. "How severely ill are you/do you find this patient?"), severity of concern score (i.e. "Are you concerned about your/her/his condition?") and loss of independency score (i.e. "Are you concerned about loss of independency after this hospital visit?" or "Do you think this patient will lose independency?"). Patients or caregivers also filled in the self-rated health score (i.e. "How would you describe your health before your visit to the ED?") while nurses and physicians answered to other questions: the 30-day SQ (i.e. "Would you be surprised if this patient died within the next 30 days?") and the length of hospital stay score (i.e. "Do you think this patient will be admitted for more than 7 days?").

Patients, nurses and physicians were able to predict 30-day mortality using their clinical intuition (AUCs ranging from 0.64 to 0.75 ). The highest discriminatory values were found for the nurses' and physicians' severity of concern score (AUCs of 0.75 ). Other studies, which mostly used clinical intuition at a later stage during the ED visit or hospital admission and used a different kind of clinical intuition score also found similar results. ${ }^{1-3}$ Interrater agreement was poor between patients and health-care professionals and fair between nurses and physicians, which was also found in a previous study. ${ }^{4}$ This poor agreement could mean that patients and health care professionals interpreted the same question in a different perspective. Health care professionals are confronted with acutely ill patients on a daily basis, which can affect their interpretation of disease severity. Interestingly, the diagnostic accuracy of the clinical intuition improved when patients/caregivers and professionals were in agreement, which definitely supports the importance of teamwork and shared decision making in ED care.

The discriminatory value for the secondary composite endpoint was lower, which may reflect the nature of the questions, which focused on the medical condition. An exception was the prediction of loss of independent living by nurses, which yielded an AUC of 0.81 . The fact that nurses are trained to assess a patient's functional status and focus on future care needs probably explains this high discriminatory value. 
A limitation of the study is that the questionnaires that were used were not validated and that the reproducibility of the scores were not assessed. In addition, it would have been interesting to test the discriminatory value of the scores in a later stage of the ED visit, i.e. after medical assessment and knowledge of the results of diagnostics.

It seems fair to conclude that clinical intuition can be used as an inexpensive tool to identify older patients at risk of adverse outcomes directly upon arrival at the ED and this may be helpful in guiding a more personalised emergency care and decision making.

\section{Concerns of patients and caregivers}

The majority of older ED patients and their caregivers (88\%) was concerned at the beginning of their ED visit. Those who were concerned were asked an open question regarding the specific nature of their concerns: "What are you concerned about?". The answers to this question are presented in chapter 4 . The concerns were divided into 10 categories and differences between patients and caregivers and between agegroups were investigated. In addition, the association between the concerns and different adverse outcomes were analysed.

Older ED patients and caregivers were particularly concerned about the severity of their disease, their functional decline and their risk of dying. Compared to other studies, our study provides more insight in the different types of concerns because other studies, which were performed in other populations and settings, did not explicitly ask the patients what they were concerned about using an open question. ${ }^{5-7}$ It is therefore possible that in these studies not all of the patients' concerns were disclosed. Since patients are often reserved to talk about their concerns to the medical staff ${ }^{8,9}$, it is important that open questions are asked to let them freely ventilate their concerns, which probably will reflect their true needs and preferences regarding medical care.

Another finding was that patients were less often concerned about their medical condition compared to their caregivers, especially regarding disease severity and cognitive decline. The nature or severity of the disease for which the patient visits the ED and, possibly, cognitive impairment could be the reasons why they assess their condition in a different way. In addition, older patients could be more at peace with their own fate than their caregivers. For clinicians, it is important to realise that concerns are different for patients and caregivers, as this may demand different kinds of (care) needs. 
Interestingly, the concern about dying was associated with 30-day mortality (OR 2.89) and with other adverse outcomes (i.e. secondary composite endpoint consisting of ICU/MCU admission, prolonged LOS, loss of independent living and unplanned readmission within 30 days after discharge, OR 2.32). In addition, unspecified concerns were associated with mortality as well (OR 1.88), which may indicate that it is difficult for patients and caregivers to discuss the concern of dying. Nevertheless, talking about a patient's concerns could provide an opportunity to talk about their preferences regarding medical and end-of-life care, and could improve shared decision making. The importance of discussing the concerns of patients and caregivers and involving them in clinical decision making, was also highlighted in a recent study about the role of a patient's intuition in decisions from the medical disciplinary boards. ${ }^{10}$ Other studies show that effective communication, pain management and involvement of caregivers help in reducing anxiety in ED patients. It may, therefore, be important to investigate whether early communication about the nature of the medical problem and treatment options would reduce the concerns and anxiety in older ED patients.

A limitation of this part of the study was that questionnaires were used while an open interview would have yielded more detailed information and could have revealed different kind of concerns. Therefore, future studies regarding this topic should use open interviews in order to further explore the different types of concerns and care needs of older ED patients.

Nevertheless, it is very important to explicitly ask our patients or caregivers about their concerns during ED stay, since this may offer opportunities to reduce anxiety and adjust care to the preferences and needs of our patients and their caregivers.

\section{Biomarkers}

The predictive value of several biomarkers with respect to 30-day mortality and other adverse outcomes is presented in chapter 6. For this part of the RISE UP study we used data of patients who were included in Zuyderland MC, since several biomarkers were routinely measured in all participants from this centre only. Five biomarkers lactate, high-sensitivity cardiac Troponin T (hs-cTnT), N-terminal pro-B-type natriuretic peptide (NT-proBNP), D-dimer and procalcitonin (PCT) - were measured in blood samples which were collected during routine blood sampling upon ED arrival. The reason to explore the value of these five biomarkers is that they are frequently used in the ED to detect underlying conditions or diseases, which are often present in the ED population. 
All biomarkers were predictive of 30-day mortality with AUCs ranging from 0.67 to 0.73 , with highest values for hs-cTnT and NT-proBNP. The predictive ability for the secondary composite endpoint (ICU/MCU admission, prolonged LOS, loss of independent living and unplanned readmission within 30 days after discharge) was lower (AUCs ranging from 0.58 to 0.67). Previous studies, which found similar associations between these biomarkers and adverse outcomes (and in particular mortality), did not routinely measure these biomarkers. ${ }^{11-22}$ Other studies, however, show that mortality rates are lowest in patients in whom biomarkers were not ordered at all ${ }^{12,23}$, which implicates that the predictive value of biomarkers is different when measured routinely. The strength of this part of the RISE UP study, therefore, is that the biomarkers were routinely measured in all older patients, which makes our findings more representative of the biomarkers' true predictive value.

Despite the fact that the five biomarkers were predictive of 30-day mortality, there was a large overlap in biomarker values between survivors and non-survivors. The overlap in biomarker values was most prominent in patients with non-extreme values, while, especially in this group of patients, the prognosis is likely to be less evident to the physician. The maximum interval likelihood ratios (LRs) for the biomarkers were moderate or only applicable to a small number of patients (lactate), which makes it unlikely that these biomarkers could contribute to clinical decision making. ${ }^{24}$ Measurement of these biomarkers is expensive, and since biomarkers were above reference ranges in most patients, they will probably lead to additional costs by performing extra diagnostic procedures (e.g. CT pulmonary angiography or echocardiography).

Limitations of this part of the study were its single centre design and the fact that the biomarkers were only measured immediately after ED arrival, while a serial biomarker measurement could have yielded more prognostic information.

Thus, lactate, hs-cTnT, NT-proBNP, D-dimer and PCT are predictive of short term mortality and other adverse outcomes in older medical ED patients, but they are unlikely to contribute to clinical decision making. Their routine measurement for prediction of mortality and other adverse outcomes is, therefore, not recommended.

In chapter 7, the risk of major adverse cardiovascular events (MACE) and mortality in older patients within 1 year after their ED visit for non-cardiac complaints, has been described. In this part of the study, the predictive ability of the cardiac biomarkers hscTnT and NT-proBNP for MACE was investigated. Both biomarkers were measured upon ED arrival in all participants from Zuyderland MC.

This study showed that $20 \%$ of the older patients are at risk of developing MACE within 1 year after their ED visit, which is a very high proportion considering that 
these patients did not present with cardiac complaints. Heart failure and CVA/TIA were the most prevalent first presentations of MACE. The high risk of MACE can be explained because cardiovascular (CV) comorbidities and risk factors were present in the majority of patients in combination with underlying chronic inflammation due to ageing and chronic diseases.

Both hs-cTnT and NT-proBNP were predictive of subsequent MACE, which is in line with other studies ${ }^{25-30}$ that were performed in other clinical settings. In addition, in contrast with hs-cTnT, NTproBNP was an independent predictor for MACE, which was found in other populations as well. ${ }^{27,28}$ That NTproBNP is an independent predictor of MACE can be explained by the finding that half of the MACE were due to heart failure and many patients had a history of heart failure. In addition, several conditions that are found to raise levels of NT-proBNP (e.g. ageing, renal failure, sepsis, shock, pulmonary embolisms) ${ }^{31}$ were present among the older ED patients.

In this cohort of older ED patients, 1-year mortality was high (32.3\%) and of those who died, $15.1 \%$ died of CV causes, which is probably an underestimation since in most patients no post mortem exams were performed. Illustrative for this possible underestimation, is that patients who developed MACE had a higher 1-year all-cause mortality (46.5\%) compared to patients who did not (28.7\%).

A limitation of this part of the study is that it was a secondary analysis of the prospective RISE UP study. For this part of the study, data regarding CV comorbidities and CV risk factors (e.g. atrial fibrillation, smoking, body mass index, hypercholesterolemia and family history of CV diseases) were assumed to be absent when not mentioned in medical files, which could have led to information bias. In addition, despite the fact that all medical files were checked carefully, it is possible that in some patients MACE was present at time of the index ED visit, while this was an exclusion criterion.

Still, older ED patients are at high risk of MACE and mortality within 1 year after the ED visit. Both hs-cTnT and NT-proBNP predict subsequent MACE in older ED patients who present with non-cardiac complaints, but only NT-proBNP was found to be an independent predictor of MACE. It is unknown whether close monitoring or interventions would improve outcome in high risk patients and this will be an important subject for future prospective studies.

\section{Development and validation of the RISE UP score}

The second aim of this thesis was to develop an easily applicable prediction model for short-term mortality as described in chapter $\mathbf{5}$. The RISE UP score was developed 
using the data of patients who were prospectively included at the EDs of Zuyderland $\mathrm{MC}$ and $\mathrm{MUMC}+$ and validated in patients who were retrospectively included in Zuyderland MC locations Heerlen and Sittard. For development of the model, candidate predictors were selected if they could be objectively and reliably retrieved during ED visit, were available in at least $90 \%$ of the participants and were considered to be generalisable. These criteria were applied with the aim to develop a model that would be easy to implement in routine ED care.

The RISE UP score that was developed in this part of the study consisted of the items age, $\geq 2$ abnormal vital signs, albumin, blood urea nitrogen (BUN), lactate dehydrogenase (LDH) and bilirubin. The score was found to have a very good discriminatory value for 30-day mortality in the derivation (AUC 0.84) and external validation cohort (AUC 0.83) and calibration was excellent.

The RISE UP score provides face validity because the items included in this model are indicators of serious underlying conditions (e.g. sepsis, malignancy, renal failure, liver disease and shock) and were found to be predictive of mortality in other studies as well. ${ }^{32-37}$ Because these items are such strong predictors of short-term mortality, this confirmed our hypothesis that disease severity at the moment of presentation to the ED is highly important in predicting short-term mortality.

The discriminatory value of the RISE UP score appeared to be significantly higher than that of other well-known scores, including the Identification of Seniors at Risk (ISAR) score, ISAR-hospitalised patients (ISAR-HP) score, Acute Physiology and Chronic Health Evaluation II (APACHE II) and Modified Early Warning Score (MEWS). In addition to the high discriminatory value, the RISE UP score is feasible for implementation in routine care because it includes items that are easily and reliably retrievable during ED stay.

A possible limitation of the RISE UP score is that the laboratory tests incorporated in the score are not routinely measured in non-medical older patients, which could reduce the general applicability of the score. However, the laboratory tests incorporated in the RISE UP score are inexpensive and easily accessible.

In conclusion, the RISE UP score is an accurate and easy to use prediction model which identifies both patients at high and low risk of dying. The score can therefore be helpful in clinical practice, by guiding decisions regarding diagnostics and treatment intensity in older ED patients.

In chapter 6, the additional value of the biomarkers lactate, hs-cTnT, NT-proBNP, Ddimer and PCT for prediction of 30-day mortality was assessed. For this substudy, we used data of patients who were recruited in Zuyderland MC only, because biomarkers were routinely assessed in these patients only. When the biomarkers were individually added to the RISE UP score (with an AUC of 0.85 in this cohort), the AUC 
only minimally improved (with a maximum AUC of 0.87 when hs-cTnT was added). Similarly, when multiple biomarkers were added the AUC only improved with 0.03 to an AUC of 0.88 when lactate, hs-cTnT and D-dimer were added at the same time. As stated before, routinely determining biomarkers is expensive and will also lead to indirect additional costs.

These high costs in combination with the minor improvement in discriminatory value confirm our earlier conclusion that routine determination of biomarkers for prediction of short-term mortality in older ED patients is not recommended, even more because the RISE UP score provides an inexpensive and accurate assessment of mortality risk in this population.

Finally, we explored the external validation of the RISE UP score in corona virus disease 2019 (COVID-19) patients as described in chapter 8. In the past year, the world has been struck with a pandemic caused by the severe acute respiratory syndrome coronavirus 2 (SARS-CoV2) which resulted in a huge burden on healthcare system. Because $70 \%$ of the admitted COVID-19 patients were 65 year or older and were severely ill at the moment of ED presentation, this led to the hypothesis that the RISE UP score could be applied in these patients as well. The discriminatory value of the RISE UP score for 30-day mortality and a composite endpoint of ICU admission and/or 30-day mortality was tested in a cohort of admitted COVID-19 patients in Zuyderland MC.

The discriminatory ability of the RISE UP score for 30-day mortality was good with an AUC of 0.77. For the composite endpoint, the AUC was somewhat lower (0.72). The discriminatory value for mortality in COVID-19 patients is lower than the AUC in the original cohort (AUC 0.83 ) which can be explained because mortality in this cohort of COVID-19 patients was much higher (26\%) compared to the original derivation (10.9\%) and validation cohort (13.3\%). Since mortality in COVID-19 patients was much higher, the model had to be recalibrated. Another explanation for the difference in AUC is that LDH is used in the RISE UP score, which is often elevated as part of the disease in COVID-19 patients. In addition, the rapid deterioration that was observed in some patients, who initially presented with only mild symptoms, could negatively affect the discriminatory value of the score. Nevertheless, the RISE UP score still has a good discriminatory value in a population that was different from the population in which it was originally designed for, which indicates that the RISE UP score reflects disease severity and could therefore be applicable to the general ED population.

In this cohort of COVID-19 patients the RISE UP score could also identify patients at low and high risk of mortality. Patients with a RISE UP score $<10 \%$ were found to be at 
very low risk of mortality with a sensitivity and negative predictive value (NPV) of $100 \%$.

Our analyses allowed us to conclude that the RISE UP score is useful in predicting mortality in COVID-19 patients as well and may be used in managing the following stage of the COVID-19 pandemic to guide clinical decision making, especially when resources are scarce.

\section{Main conclusions of the thesis}

In this thesis, several tools for predicting adverse outcome, and in particular 30-day mortality, in older ED patients, were investigated. Currently, adequate tools to predict prognosis are lacking and most geriatric assessments take too much time in the busy, often crowded ED and require information that is not reliably available right away. For these reasons, this thesis focussed on tools that use items that are readily available and reliably retrievable during ED stay. Because mortality plays such a strong role in clinical decision making in older patients ${ }^{38,39}$, the focus of this thesis was 30 -day mortality.

The following main conclusions can be drawn from this thesis. First, clinical intuition, the concerns of patients and caregivers, biomarkers and the newly developed simplified prediction model, the RISE UP score, can all predict 30-day mortality and adverse outcomes in medical ED patients. Second, the RISE UP score outperforms other subjective (i.e. clinical intuition and concerns) and more expensive (i.e. biomarkers) tools and established risk-stratification scores (i.e. ISAR score, ISAR-HP, APACHE II score and MEWS) and can also be used for risk prediction in COVID-19 patients. Third, the added value of biomarkers is only minimal. This can be explained because the RISE UP score already has a high predictive ability and other predictors can only slightly improve the performance of a model that has already good discriminatory power in itself. ${ }^{40}$ Another explanation for the limited additional value might be that both the five biomarkers and the items in the RISE UP score reflect disease severity. Disease severity is probably such a strong predictor of mortality, because it reflects both the pre-morbid condition and the acute disease for which the patient contacts the ED.

In conclusion, several tools predict adverse outcome in medical ED patients, but for 30-day mortality, the RISE UP score is preferred because it is an inexpensive, objective and easily applicable tool with good discriminatory value. 


\section{Strengths, limitations and future perspectives}

The strengths of the RISE UP study are its prospective multicentre design and the inclusion of older patients with diverse complex medical problems. Moreover, a large variety of early predictors for adverse outcome were investigated. As our study was conducted in one of the regions in Europe with the fastest ageing population, the RISE UP study is representative for an ageing population.

Apart from the limitations that were already alluded to earlier, some other limitations apply to the RISE UP study. First, not all older patients who visited the ED were included in the study because physicians had to give priority to providing emergency care in times of crowding. Not including all patients could have led to inclusion bias. However, possible inclusion bias was investigated by comparing baseline characteristics and outcome of included and non-included patients and this did not show any evidence for such bias. The problem of not being able to include every candidate in an emergency care study is possibly unavoidable, since EDs are often crowded and a physicians' top priority is providing medical care first.

Second, in the RISE UP study, only medical (i.e. internal medicine/gastroenterology) patients were included, and it is possible that the results cannot be extrapolated to older ED patients who present for non-medical (i.e. surgical) specialties. Older medical patients were selected because they represent a large group of patients with complex problems and comorbidities, often accompanied by frailty, with a high risk of adverse outcomes. It will be interesting for future studies to focus on validating the RISE UP score in different populations and settings, in order to assess its generalisability.

Third, the RISE UP score uses a complex formulae to give an estimation of the risk of mortality instead of designating a patient into high or low risk, which could make the score less feasible to use. To improve feasibility, the RISE UP score is already incorporated in an online calculator and could also be incorporated into an electronic medical record system or app in the future. This will make the score easier to use in clinical practice and in future research.

Finally, one major question remains unanswered after completion of this thesis: "How does the prognosis of patient affect clinical decision making and medical care?". The influence of prediction of prognosis by the RISE UP score on clinical decision making was not studied in this thesis. Another issue is that although a tool can accurately predict prognosis in a group of patients, it still remains very difficult to translate this prognosis to the individual patient. 
The answers to these issues may be found in the importance of prognosis for a clinician, patient and/or caregiver. The prognosis of a patient will most likely play an crucial role in clinical decision making, whether or not unwittingly, by affecting a clinician's awareness. ${ }^{38,39,41,42}$ Clinicians can place a patient's prognosis in the whole context of the individual patient, e.g. his or her comorbidities, cognitive and functional status, medical problem and preferences regarding care. A high risk of mortality after screening may therefore result in the decision to intensify medical care or in the decision not to apply certain (invasive) diagnostics or treatments. Prediction of prognosis will provide an opportunity to focus on the quality of life rather than quantity of life, which could be more important for older patients. ${ }^{43}$ Despite the obligation of a physician to 'first do no harm', the decision to hold back certain (aggressive) diagnostics or treatments is often much harder to make than the decision to treat maximally. ${ }^{44}$ This is especially true when major decisions regarding medical care have to be made within the chaotic and noisy ED environment, when dealing with unfamiliar patients who often present with non-specific complaints. The experience of clinicians and his or her curiosity to find out what causes the complaints of the patient will also affect the decisions.

Despite the role of prognosis in clinical decision making, the preferences of a patient (or their caregivers) are crucial. That is why it is so important to listen to our patients and explicitly ask about their concerns, needs, preferences and goals regarding medical care. A physician should therefore not only focus on what kind of treatment options are necessary or possible, but more importantly, find time to discuss what kind of care a patients desires. Knowledge on the prognosis can be used to guide this conversation, but is not always necessary (e.g. when a patient prefers no medical treatment).

In conclusion, prognosis can be used in clinical decision making and in the conversation regarding the patient's preferences and needs of medical care. However, prospective randomised impact studies are needed to determine how prediction of prognosis will lead to personalisation of emergency care and improvement of outcome or wellbeing. 


\section{References}

1. Brabrand $\mathrm{M}$, Hallas J, Knudsen T. Nurses and physicians in a medical admission unit can accurately predict mortality of acutely admitted patients: a prospective cohort study. PloS One 2014;9(7): e101739.

2. Beglinger B, Rohacek M, Ackermann S, et al. Physician's first clinical impression of emergency department patients with nonspecific complaints is associated with morbidity and mortality. Medicine (Baltimore) 2015;94(7):e374.

3. White N, Kupeli N, Vickerstaff V, et al. How accurate is the 'Surprise Question' at identifying patients at the end of life? A systematic review and meta-analysis. BMC Med 2017;15(1):139-39.

4. Rohacek $\mathrm{M}$, Nickel $\mathrm{CH}$, Dietrich $\mathrm{M}$, et al. Clinical intuition ratings are associated with morbidity and hospitalisation. Int J Clin Pract 2015;69(6):710-7.

5. Andersson-Segesten K, Erichsen M, Westerlund A, et al. Patients' fears, worries, and concerns when visiting an out-patient clinic. Scand J Primary Health Care 1989;7(4):197-201.

6. Olthuis G, Prins C, Smits MJ, et al. Matters of concern: a qualitative study of emergency care from the perspective of patients. Ann Emerg Med 2014;63(3):311-9.e2.

7. White $M$, Edmondson $D$, Umland $R$, et al. Patient perceptions of stress during evaluation for ACS in the ED. Am J Emerg Med 2017;35(2):351-52.

8. Baraff LJ, Bernstein E, Bradley K, et al. Perceptions of emergency care by the elderly: results of multicenter focus group interviews. Ann Emerg Med 1992;21(7):814-8.

9. Lim C, Berry ABL, Hirsch T, et al. "It just seems outside my health": How Patients with Chronic Conditions Perceive Communication Boundaries with Providers. DIS Designing Interactive Systems (Conference) 2016;2016:1172-84.

10. Schuck UMvdW, M.W.J.; Dinant, G.J.; Stolper, C.F. Horen artsen intuïtie van patiënten serieus te nemen? Ned Tijschr Geneeskd 2020(164):D4884.

11. de Groot B, Verdoorn RC, Lameijer J, et al. High-sensitivity cardiac troponin $T$ is an independent predictor of inhospital mortality in emergency department patients with suspected infection: a prospective observational derivation study. Emerg Med J 2014;31(11):882-8.

12. Courtney D, Conway R, Kavanagh J, et al. High-sensitivity troponin as an outcome predictor in acute medical admissions. Postgrad Med J 2014;90(1064):311-6.

13. Nickel $\mathrm{CH}$, Kuster T, Keil C, et al. Risk stratification using D-dimers in patients presenting to the emergency department with nonspecific complaints. Eur J Intern Med 2016;31:20-4.

14. Rodelo JR, De la Rosa G, Valencia ML, et al. D-dimer is a significant prognostic factor in patients with suspected infection and sepsis. Am J Emerg Med 2012;30(9):1991-9.

15. Wilhelm J, Hettwer S, Hammer D, et al. Outcome prediction using clinical scores and biomarkers in patients with presumed severe infection in the emergency department. Med Klin Intensivmed Notfmed 2012;107(7):558-63.

16. Kruse O, Grunnet N, Barfod C. Blood lactate as a predictor for in-hospital mortality in patients admitted acutely to hospital: a systematic review. Scand J Trauma Resusc Emerg Med 2011;19:74.

17. Park YJ, Kim DH, Kim SC, et al. Serum lactate upon emergency department arrival as a predictor of 30day in-hospital mortality in an unselected population. PloS One 2018;13(1):e0190519.

18. Puskarich MA, Illich BM, Jones AE. Prognosis of emergency department patients with suspected infection and intermediate lactate levels: a systematic review. J Crit Care 2014;29(3):334-9.

19. del Portal DA, Shofer F, Mikkelsen ME, et al. Emergency department lactate is associated with mortality in older adults admitted with and without infections. Acad Emerg Med 2010;17(3):260-8.

20. Marchetti M, Benedetti A, Mimoz O, et al. Predictors of 30-day mortality in patients admitted to ED for acute heart failure. Am J Emerg Med 2017;35(3):444-47.

21. Meyer $B$, Huelsmann $M$, Wexberg $P$, et al. N-terminal pro-B-type natriuretic peptide is an independent predictor of outcome in an unselected cohort of critically ill patients. Crit Care Med 2007;35(10):2268-73.

22. Zhao Y, Li C, Jia Y. Evaluation of the Mortality in Emergency Department Sepsis score combined with procalcitonin in septic patients. Am J Emerg Med 2013;31(7):1086-91. 
23. van den Nouland DP, Brouwers MC, Stassen PM. Prognostic value of plasma lactate levels in a retrospective cohort presenting at a university hospital emergency department. BMJ Open 2017;7(1):e011450.

24. Grimes DA, Schulz KF. Refining clinical diagnosis with likelihood ratios. Lancet (London, England) 2005;365(9469):1500-5.

25. Aimo A, Januzzi JL, Jr., Vergaro G, et al. High-sensitivity troponin T, NT-proBNP and glomerular filtration rate: A multimarker strategy for risk stratification in chronic heart failure. Int J Cardiol 2019;277:166-72.

26. Zhang S, Zhang HX, Lin RY, et al. Predictive role of NT-pro BNP for adverse cardiac events in community-acquired pneumonia: A retrospective study. 2016;9:14411-17.

27. Onda T, Inoue K, Suwa S, et al. Reevaluation of cardiac risk scores and multiple biomarkers for the prediction of first major cardiovascular events and death in the drug-eluting stent era. Int J Cardiol 2016;219:180-5.

28. Satyan S, Light RP, Agarwal R. Relationships of N-terminal pro-B-natriuretic peptide and cardiac troponin $\mathrm{T}$ to left ventricular mass and function and mortality in asymptomatic hemodialysis patients. Am J Kidney Dis 2007;50(6):1009-19.

29. Leistner DM, Klotsche J, Pieper L, et al. Prognostic value of NT-pro-BNP and hs-CRP for risk stratification in primary care: results from the population-based DETECT study. Clin Res Cardiol 2013;102(4):259-68.

30. Zhang L-J, Li N, Li Y, et al. Cardiac Biomarkers Predicting MACE in Patients Undergoing Noncardiac Surgery: A Meta-Analysis. Front Physiol 2019;9:1923-23.

31. Tsai SH, Lin YY, Chu SJ, et al. Interpretation and use of natriuretic peptides in non-congestive heart failure settings. Yonsei Med J 2010;51(2):151-63.

32. de Gelder J, Lucke JA, de Groot B, et al. Predicting adverse health outcomes in older emergency department patients: the APOP study. Neth J Med 2016;74(8):342-52.

33. Ha DT, Dang TQ, Tran NV, et al. Development and validation of a prognostic model for predicting 30day mortality risk in medical patients in emergency department (ED). Sci Rep 2017;7:46474.

34. Buurman BM, van Munster BC, Korevaar JC, et al. Prognostication in acutely admitted older patients by nurses and physicians. J Gen Intern Med 2008;23(11):1883-9.

35. Jellinge ME, Henriksen DP, Hallas $P$, et al. Hypoalbuminemia is a strong predictor of 30-day all-cause mortality in acutely admitted medical patients: a prospective, observational, cohort study. PloS One 2014;9(8):e105983.

36. de Gelder J, Lucke JA, Heim N, et al. Predicting mortality in acutely hospitalized older patients: a retrospective cohort study. Intern Emerg Med 2016;11(4):587-94.

37. Loekito E, Bailey J, Bellomo R, et al. Common laboratory tests predict imminent death in ward patients. Resuscitation 2013;84(3):280-5.

38. Gill TM. The Central Role of Prognosis in Clinical Decision Making. JAMA 2012;307(2):199-200.

39. Yourman LC, Lee SJ, Schonberg MA, et al. Prognostic indices for older adults: a systematic review. JAMA 2012;307(2):182-92.

40. Pencina MJ, D'Agostino RB, Pencina KM, et al. Interpreting incremental value of markers added to risk prediction models. Am J Epidemiol 2012;176(6):473-81.

41. Gospodarowicz M, Mackillop W, O'Sullivan B, et al. Prognostic factors in clinical decision making. Cancer 2001;91(S8):1688-95.

42. van Dam CS, Trappenburg MC, Peters MJL, et al. Screening for vulnerability in older adults attending the emergency department. Ned Tijdschr Geneeskd 2019;163.

43. Westendorp RGJ, Kusumastuti S. Screening tools do not measure up against rigorous history taking. Ned Tijdschr Geneeskd 2019;163

44. Bookman K, Abbott J. Ethics seminars: withdrawal of treatment in the emergency department--when and how? Acad Emerg Med 2006;13(12):1328-32. 

Samenvatting 



\section{Samenvatting}

Met de toenemende levensverwachting stijgt ook de zorgbehoefte bij de ouderen ( $\geq 65$ jaar) wereldwijd. Hierdoor zal ook het aantal ouderen dat de spoedeisende hulp (SEH) bezoekt stijgen, juist omdat de SEH een belangrijke rol speelt in het verlenen van acute zorg voor ouderen. De zorg op de SEH wordt steeds complexer mede doordat oudere patiënten zich vaak presenteren met multipele problemen waarbij op de SEH belangrijke beslissingen over diagnostiek, behandelingen en eventuele beperkingen daarin, moeten worden genomen in een kort tijdsbestek. Deze beslissingen worden bemoeilijkt doordat meerdere, vaak tevoren onbekende patiënten zich tegelijkertijd presenteren op de SEH waardoor zorgverleners meerdere taken tegelijk moeten uitvoeren.

$\mathrm{Na}$ een SEH-bezoek hebben oudere patiënten ( $\geq 65$ jaar) een grote kans op ongunstige uitkomsten zoals ziekenhuisopname, opname op een intensive care (IC) of medium care (MC) afdeling, heropname, functieverlies (cognitief of fysiek) en/of overlijden. Vroegtijdige herkenning van ouderen met een verhoogd risico op deze ongunstige uitkomsten is van groot belang, zodat de zorg tijdig kan worden aangepast met als doel de zorg, uitkomst en/of levenskwaliteit van de patiënt te verbeteren. Om het risico op een ongunstige uitkomst te kunnen inschatten kunnen scores die prognose voorspellen worden gebruikt. Deze scores worden risicostratificatie scores genoemd. De risicostratificatie scores die tot voor kort ontwikkeld zijn, zijn echter niet betrouwbaar genoeg in het inschatten van het risico op ongunstige uitkomsten óf vereisen informatie die moeilijk betrouwbaar te verzamelen is op een SEH (zoals cognitief of lichamelijk functioneren).

Het doel van dit proefschrift was dan ook om betrouwbare en makkelijk beschikbare middelen te vinden die het risico op een ongunstige uitkomst kunnen voorspellen, teneinde een betrouwbare en makkelijk toepasbare risicostratificatie score te ontwikkelen. Hiervoor werd de 'RIsicoStratificatiE bij acUut zieke oudere Patiënten (RISE UP)' studie opgezet, die plaatsvond op de SEHs van het Zuyderland Medisch Centrum (MC) en Maastricht Universitair Medisch Centrum+ (MUMC+). In de periode van juli 2016 t/m februari 2017 werden oudere SEH-patiënten ( $\geq 65$ jaar), die behandeld werden door de internist of maag-darm-lever (MDL) arts, geïncludeerd in de studie. Het studieprotocol van de RISE UP studie wordt beschreven in hoofdstuk 2. In de rest van dit proefschrift worden de resultaten van deze studie beschreven. 


\section{Patiëntenpopulatie}

In de RISE UP studie werden in totaal 603 patiënten geïncludeerd met een gemiddelde leeftijd van 79 jaar waarbij de meeste patiënten nog zelfstandig woonden. Ongeveer $11 \%$ van de patiënten overleed binnen 30 dagen na het SEH bezoek en een groot deel van de patiënten (45\%) kreeg te maken met een andere ongunstige uitkomst binnen 30 dagen, zoals IC/MC opname, langdurige ziekenhuisopname (>7 dagen), ontslag naar verzorgings- of verpleeghuis of heropname.

\section{Vroege voorspellers van ongunstige uitkomsten bij ouderen}

In de RISE UP studie werden verschillende vroege voorspellers van ongunstige uitkomsten onderzocht die we in volgende 3 categorieën kunnen indelen: 1) de klinische blik van patiënten, verpleegkundigen en artsen, 2) de zorgen van patiënten en hun begeleiders en 3) biomarkers (verschillende factoren die meetbaar zijn in het bloed en specifieke onderliggende aandoeningen kunnen aantonen).

\section{Klinische blik van patiënten, verpleegkundigen en artsen}

Verpleegkundigen en artsen hebben vaak een 'onderbuikgevoel' dat ook wel 'klinische blik' genoemd wordt. Het is een intrinsiek gevoel of er al dan niet iets mis is met de patiënt. Het is heel goed mogelijk dat patiënten en hun begeleiders dit gevoel ook ervaren. In hoofdstuk 3 van dit proefschrift werd de voorspellende waarde van de klinische blik beschreven voor overlijden en andere ongunstige uitkomsten (samengesteld eindpunt bestaande uit IC/MC opname, langdurige ziekenhuisopname, ontslag naar verzorgings- of verpleeghuis en heropname) binnen 30 dagen na SEHbezoek. Om dit te kunnen onderzoeken hebben patiënten, verpleegkundigen en artsen aan het begin van het SEH-bezoek een vragenlijst ingevuld. Er werd gebruik gemaakt van verschillende vragen die de ernst van ziekte, de mate van bezorgdheid en de mate van gezondheid voorafgaand aan het SEH-bezoek weerspiegelden.

Zowel patiënten, verpleegkundigen als artsen waren redelijk in staat om met behulp van hun klinische blik de kans op overlijden te voorspellen waarbij de zorgverleners dit het best konden voorspellen. Er was een redelijke overeenkomst in de klinische blik van artsen en verpleegkundigen terwijl er grote verschillen werden gezien tussen patiënten en zorgverleners. De klinische blik bleek het best voorspellend te zijn als patiënt, verpleegkundige en arts dezelfde voorspelling deden, wat het belang van het betrekken van patiënten (en hun eventuele begeleiders) in zorgkeuzes benadrukt. Ook 
de andere ongunstige uitkomsten konden met behulp van de klinische blik worden voorspeld, maar wel minder goed dan sterfte.

De klinische blik kan dus gebruikt worden in het voorspellen van overlijden en andere ongunstige uitkomsten bij acuut zieke ouderen op de SEH en kan meewegen in de besluitvorming op de SEH.

\section{Zorgen van de patiënt en begeleider}

Er is weinig bekend over de verschillende zorgen van patiënten en begeleiders tijdens een SEH bezoek en de mogelijke voorspellende waarde van deze zorgen voor de gerelateerde uitkomst. In hoofdstuk 4 werden de verschillende type zorgen van patiënten en begeleiders beschreven en hun voorspellende waarde voor overlijden en andere ongunstige uitkomsten. In een vragenlijst werden patiënten (of hun begeleiders) naar hun zorgen gevraagd. De antwoorden op de vraag werden ingedeeld in 10 verschillende categorieën.

De meeste oudere patiënten of hun begeleiders (88\%) waren bezorgd ten tijde van het SEH-bezoek waarbij de meesten bezorgd waren over de ernst van de ziekte, functionele achteruitgang of overlijden. Patiënten bleken minder vaak bezorgd te zijn dan hun begeleiders, met name als het ging om de van ernst van de ziekte en/of cognitieve achteruitgang. Er waren geen verschillen tussen de zorgen van patiënten in verschillende leeftijdsgroepen. Bezorgdheid over overlijden was geassocieerd met een verhoogde kans op overlijden en andere ongunstige uitkomsten binnen 30 dagen na SEH-bezoek. Verrassend genoeg bleek dat de kans op overlijden eveneens groter was wanneer de patiënt of begeleider bezorgd waren maar hun zorgen niet konden specificeren. Aangezien een onbestemd gevoel eveneens voorspellend is voor ongunstige uitkomsten, is het belangrijk om patiënten actief te vragen naar hun zorgen en deze zorgen bespreekbaar te maken.

De resultaten van deze studie laten zien dat het belangrijk is om SEH-patiënten specifiek naar zorgen te vragen, ook al zijn deze zorgen onbestemd. Door dit bespreekbaar te maken, kunnen de zorgverleners manieren vinden om hierop in te spelen en zo mogelijk de zorg aan te passen aan de behoeften van de patiënt.

\section{Biomarkers}

Verschillende biochemische factoren die in het lichaam aanwezig zijn en samenhangen met specifieke onderliggende ziekten of aandoeningen worden ook wel 'biomarkers' genoemd. Biomarkers die op de SEH vaker bepaald worden zijn lactaat, high-sensitivity cardiac Troponine $\mathrm{T}$ (hs-cTnT), N-terminal pro-B-type natriuretic peptide (NT-proBNP), D-dimeer en procalcitonine. Lactaat (melkzuur) is een marker 
voor zuurstoftekort in de weefsels, hetgeen kan wijzen op een laag zuurstofgehalte in het bloed (hypoxemie) of op een verminderde doorbloeding van weefsels (bijv. bij shock of vaatvernauwing). Hs-cTnT en NT-proBNP worden frequent gebruikt als hulpmiddel bij het aantonen of uitsluiten van bepaalde hartziekten zoals hartinfarct of hartfalen. Bepaling van D-dimeren wordt toegepast in de analyse van mogelijke stolselvorming in de vaten, bijvoorbeeld bij verdenking op een trombosebeen of longembolie. Procalcitonine is een factor die voorspellend kan zijn voor een bacteriële infectie als oorzaak van het ziektebeeld. Deze bovengenoemde aandoeningen zijn vaak, ook in combinatie, aanwezig bij oudere SEH patiënten en zijn allemaal geassocieerd met een hoger risico op overlijden. De voorspellende waarde van de 5 genoemde biomarkers werd beschreven in hoofdstuk 6.

Bij patiënten die geïncludeerd waren in Zuyderland $M C$ werd bloed afgenomen bij binnenkomst op de SEH. Alle 5 de biomarkers bleken redelijke voorspellers te zijn voor overlijden binnen 30 dagen na SEH bezoek en voor andere ongunstige uitkomsten (IC/MC opname, langdurige ziekenhuisopname, ontslag naar verzorgings- of verpleeghuis en heropname). Echter de voorspellende waarde van de biomarkers was vooral goed bij extreem hoge waarden, wat enkel van toepassing was op een klein aantal van de patiënten. Tevens brengt het routinematig bepalen van biomarkers significante directe kosten met zich mee en zullen afwijkende waarden zelfs leiden tot indirecte kosten in de vorm van aanvullende diagnostiek. De routinematige bepaling van deze biomarkers om overlijden te voorspellen wordt daarom niet door ons aangeraden.

Hart- en vaatziekten (ook wel cardiovasculaire ziekten genoemd) zijn wereldwijd een belangrijke doodsoorzaak bij ouderen. In de RISE UP studie werd daarom gekeken naar het ontstaan van cardiovasculaire aandoeningen binnen 1 jaar na SEH-bezoek. In hoofdstuk 7 werd het risico op majeure cardiovasculaire events (MACE) en overlijden binnen 1 jaar beschreven voor oudere patiënten die zich presenteerden op de SEH zonder hartklachten. Tevens werd onderzocht of biomarkers hs-cTnT en NT-proBNP (markers van onderliggende hartziekten) voorspellend waren voor MACE.

Dit deel van de RISE UP studie liet zien dat ongeveer $20 \%$ van de oudere SEHpatiënten binnen 1 jaar een MACE doormaakt. Hoge afwijkende waarden van hs-cTnT en NT-proBNP bleken voorspellend voor MACE binnen 1 jaar na SEH-bezoek. Hierbij was met name de hoogte van het NT-proBNP een onafhankelijke voorspeller voor MACE, wanneer er gecorrigeerd werd voor bestaande cardiovasculaire risicofactoren. Het risico op overlijden bleek hoog aangezien $32.3 \%$ van de patiënten kwam te overlijden binnen 1 jaar na het SEH-bezoek. Van deze overlijdens was $15.1 \%$ zeker toe te schrijven aan cardiovasculaire aandoeningen. Vermoedelijk is dit laatste getal nog 
een onderschatting van de werkelijkheid omdat er weinig specifieke informatie (zoals obducties) beschikbaar was omtrent de doodsoorzaak.

De resultaten van dit onderzoek zijn belangrijk omdat we hieruit kunnen afleiden dat hoog risicopatiënten wellicht baat zouden kunnen hebben bij een (preventieve) risicoanalyse, behandeling of intensieve follow up om zodoende de kans op MACE te verkleinen. Dit zou een zeer relevant onderwerp kunnen zijn voor prospectieve studies.

\section{Ontwikkeling en validatie van de RISE UP score}

Het tweede doel van dit proefschrift was het ontwikkelen van een betrouwbare en praktische risicostratificatie score om overlijden bij oudere SEH-patiënten te kunnen voorspellen. De ontwikkeling van de RISE UP score werd beschreven in hoofdstuk 5. De score werd ontwikkeld met behulp van gegevens van patiënten die prospectief geïncludeerd werden in de RISE UP studie en werd nadien getest in een onafhankelijke patiëntenpopulatie. Variabelen die gemakkelijk en betrouwbaar konden worden verzameld op de SEH en in meer dan $90 \%$ van de patiënten beschikbaar bleken te zijn werden gebruikt als mogelijke voorspellers in het model.

De RISE UP score die ontwikkeld werd bestond uit volgende variabelen: leeftijd, $\geq 2$ afwijkende vitale parameters (waaronder bloeddruk, hartfrequentie, zuurstof saturatie, ademhalingsfrequentie en mate van bewustzijn) en laboratoriumwaarden albumine, ureum, lactaat dehydrogenase (LDH) en bilirubine. De voorspellende waarde van de RISE UP score bleek goed te zijn met betrekking tot overlijden binnen 30 dagen, hetgeen bevestigd werd in de validatie populatie. De RISE UP score discrimineert in $83 \%$ van de gevallen goed tussen overleven en overlijden.

De zes variabelen in de RISE UP score hadden allen een bekende associatie met onderliggende aandoeningen en bleken in onze studie duidelijk voorspellend voor de ernst van het ziektebeeld. Tot onze verrassing liet de RISE UP score een betere voorspellende waarde zien in vergelijking met andere reeds geaccepteerde en toegepaste risicostratificatie scores, waaronder de Identification of Seniors at Risk (ISAR) score, ISAR-hospitalised patients (ISAR-HP) score, Acute Physiology and Chronic Health Evaluation II (APACHE II) en Modified Early Warning Score (MEWS).

Daarom concluderen we dat de RISE UP score een makkelijk toepasbare en betrouwbare risicostratificatie score is, die patiënten met laag en hoog risico op overlijden kan identificeren en daarom behulpzaam kan zijn in de besluitvorming op de SEH. 
In hoofdstuk 6 werd de toegevoegde waarde van de biomarkers (lactaat, hs-cTnT, NTproBNP, D-dimeer en PCT) aan het RISE UP model beschreven. Voor dit onderdeel van de studie werden data gebruikt van patiënten die geïncludeerd waren in Zuyderland MC. De voorspellende waarde van de RISE UP score nam slechts minimaal toe wanneer er 1 of meerdere biomarkers werden toegevoegd. Bepaling van biomarkers is kostbaar en zal indirect ook kunnen leiden tot extra zorgkosten, in de vorm van aanvullende diagnostiek. De hoge additionele kosten in combinatie met de marginale toename in voorspellende waarde ondersteunen onze eerdere conclusie dat routinematige bepaling van biomarkers om overlijden te voorspellen bij deze patiëntenpopulatie af te raden is.

De validatie van de RISE UP score in patiënten met corona virus disease 2019 (COVID19) werd beschreven in hoofdstuk 8. Het afgelopen jaar heeft de wereld te maken gekregen met een pandemie die wordt veroorzaakt door het severe acute respiratory syndrome coronavirus (SARS-CoV2), wat een enorme belasting op ons zorgsysteem legt. Omdat uit de gegevens van de eerste corona-golf bleek dat $70 \%$ van de opgenomen COVID-19 patiënten 65 jaar of ouder was en ernstig ziek bleken te zijn, ontstond de hypothese dat de RISE UP score wellicht ook toepasbaar zou kunnen zijn om overlijden binnen 30 dagen en IC opname in deze patiëntencategorie te voorspellen. Hiervoor werden gegevens gebruikt van patiënten die opgenomen waren met COVID-19 in het Zuyderland MC. De RISE UP score bleek overlijden binnen 30 dagen in COVID-19 patiënten goed te kunnen voorspellen. De voorspellende waarde voor het samengesteld eindpunt overlijden en IC opname was eveneens goed maar wel iets lager dan voor overlijden apart. De voorspellende waarde van het RISE UP model voor COVID-19 was echter wel iets lager dan in de ontwikkelingspopulatie, wat kan komen doordat de kans op overlijden bij patiënten met COVID-19 veel hoger was $(26 \%)$ in vergelijking met de ontwikkelings- en validatie populatie (11-13\%). Een andere verklaring waarom de voorspellende waarde van de RISE UP score lager is in COVID-19 patiënten is de observatie dat veel patiënten die stabiel binnen kwamen in zeer korte tijd onverwacht klinisch verslechteren, terwijl dit bij andere ziektebeelden minder vaak het geval is.

Wij stellen dan ook dat de RISE UP score bruikbaar kan zijn in de klinische besluitvorming gedurende de COVID-19 pandemie. 


\section{Toekomstvisie}

Een van de grote vragen die nog overblijft na het schrijven van dit proefschrift is of het voorspellen van overlijden een werkelijk effect heeft op de besluitvorming op de SEH en resulteert in een aanpassing van diagnostiek of beleid én of dit werkelijk effect heeft op de zorgkwaliteit. Dit werd aanvullend beschreven en in een breder perspectief geplaatst in hoofdstuk 9. Aanvullende prospectieve onderzoeken zullen nodig zijn om deze vraag te beantwoorden. 

Impact paragraph 



\section{Scientific and social impact of this thesis}

In this chapter, the relevance of the results described in this thesis and their scientific and social impact will be discussed.

\section{Scientific impact of the thesis}

This thesis has added value to clinical research as it provides insights in the characteristics of the older medical emergency department (ED) population, their risks of adverse outcomes and it offers new tools to identify older patients with high risks of adverse outcomes. In addition, it provides information regarding the concerns of older patients (or their caregivers) during an ED visit.

\section{Tools to predict short-term adverse outcomes}

This thesis shows that different tools, which can easily be applied during ED stay, are good predictors of adverse outcomes in older patients. This main finding has impact on ED research in several ways.

First, several questions which are depicted in five point scores, quantify clinical intuition parameters and are applicable to predict short-term mortality. This approach may be helpful in future studies regarding clinical intuition.

Secondly, the five biomarkers lactate, high-sensitivity cardiac troponin T (hs-cTnT), Nterminal pro-B-type natriuretic peptide (NT-proBNP), D-dimer and procalcitonin (PCT) are good predictors of short-term outcomes. Nevertheless, there is a large overlap in survivors and non-survivors making these biomarkers less ideal as a single predictor of mortality. Moreover, they are expensive and have limited added value to the RISE UP score. Therefore, these biomarkers should not be used solely as predictors of adverse outcome in future research. Our advice is that these parameters must be applied in clinical research as prognostic markers only on strict indication.

Thirdly, the RIsk Stratification in the Emergency department in acUtely III older Patients (RISE UP) score can predict short-term mortality accurately in older ED patients and outperforms established risk-stratification scores. Additionally, we showed that this score is also suitable for prediction of adverse outcomes in patients with COVID-19, which may be extremely valuable in decision making in the ED in the 
current pandemic and for all clinical research regarding this new infectious disease. This score has the advantage of using objective and inexpensive variables, which are part of routine work-up in the ED, making the RISE UP score a valuable tool for prediction of short-term mortality in future (ED) studies.

All parameters investigated in the RISE UP study represent disease severity, reflecting both the pre-morbid state and acute medical problems for which the patient contacts the ED. This implies that for prediction of short-term outcomes, and especially mortality, tools that represent disease severity can be used instead of geriatric variables (e.g. cognitive or physical function). This has a major impact on clinical research, since, in most ED studies regarding older patients, it remains difficult to accurately assess important geriatric aspects. The tools investigated in this thesis can therefore be used in future studies as predictors of mortality and as a reflection of disease severity, with the RISE UP score being the preferred tool.

\section{Concerns of older patients and caregivers}

This thesis has impact on qualitative research regarding concerns and care needs of older ED patients, as it provides insight in the different kinds of concerns older patients have. This knowledge can be used in future studies regarding the quality of ED care and can focus in further exploring the different kinds of concerns and care needs of older ED patients.

\section{Prediction of major adverse cardiovascular events}

The finding that older patients visiting the ED with non-specific complaints are at very high risk of developing MACE within 1 year after their ED visit has impact on cardiovascular research. Especially NT-proBNP can be used as a predictor of MACE in addition to classical cardiovascular risk factors. It would be very interesting for future research to focus on preventive interventions, e.g. preventive medications, echocardiography or close monitoring of high risk patients by cardiologists in order to reduce their risk of MACE. 


\section{Social impact of the thesis}

\section{Relevance for health care professionals, patients and caregivers}

The research findings described in this thesis are important for daily practice for health care providers since it provides insight into the risk factors of adverse outcomes in older ED patients. The finding that several parameters concerning disease severity are highly important for the prediction of short-term outcome is important for clinicians working inside as well as outside the hospital (e.g. primary care or nursing homes) because this may be very helpful in clinical decision making. The RISE UP score can be a valuable tool in the ED for health care professionals as well as for patients and caregivers since the score gives an accurate prediction of shortterm mortality. Prognosis will most likely play an important role in clinical decision making since clinicians can place a prognosis in the whole context of the individual patient, making care more personalised. Furthermore, this prognosis can be used in a conversation with patients and caregivers and may guide shared-decision making. In patients with high risk of mortality this can result in the decision to intensify medical care or, adversely, in the decision to refrain from further diagnostics or treatments and choose for supportive care. On the other hand, in patients with low risk of mortality, this prognosis can result in the decision to safely treat at home or discharge the patient in an early stage. Prediction by the RISE UP score may have impact on ED and hospital care regarding outcomes or wellbeing of older patients and reduction of costs for clinical healthcare.

This thesis also provided more information about the specific concerns of older ED patients, which is valuable to health care providers, patients and caregivers. Based on the findings provided in this thesis, it is recommended that at the ED, more attention should be paid to the concerns of the patients and their caregivers. By doing so, we will be able to find out what matters to these patients and find ways to reduce their concerns and adjust care to their needs and preferences, which will subsequently improve ED care.

\section{Relevance for society}

Prediction of mortality or other adverse clinical outcomes in older patients visiting the ED is extremely important for clinical decision making and saving of costs for health care. Moreover, in the current COVID-19 pandemic, resources turn out to be even more scarce and difficult decisions have to be made concerning treatment and allocation of medical care. For example, prediction of a low risk of mortality may 
result in a safe decision to treat patients at home (or at a corona hotel), or may result in a shorter hospital stay, while for high risk patients, it can result in the decision to transfer to the ICU at an early stage or in the decision or choice for supportive care.

\section{Implementation in daily practice}

The results of this thesis can and will be shared with others in several ways. First, by publishing the results of the studies in medical journals, healthcare providers can be informed. Secondly, we intend to publish our results on a website of Maastricht University Medical Centre+ so these will be easily accessible for other healthcare providers, patients and caregivers. Third, the RISE UP score can be implemented in routine care by development of an online calculator (which is already available) and/or by incorporating the score into an electronical medical record system. Fourth, other hospitals can and will be informed of the results of this thesis by presenting the results at scientific meetings, congresses or webinars also with the aim of collaboration in future research and medical care. 
Dankwoord 



\section{Dankwoord}

Ongeveer 5 jaar geleden ben ik begonnen met het wetenschappelijk traject wat geleid heeft tot het schrijven van dit proefschrift. Ik heb hier de afgelopen jaren met heel veel plezier aan gewerkt en heb ontzettend veel geleerd. Jaren geleden had ik nooit gedacht dat ik deze grote stap zou durven nemen. Gelukkig bleken het vele kleine stapjes die genomen moesten worden, stapjes die ik nooit had kunnen zetten zonder hulp van een aantal mensen die ik graag wil bedanken:

Ten eerste alle proefpersonen (en hun begeleiders) die hebben deelgenomen aan de studie. Ontzettend bedankt voor jullie welwillendheid om mee te werken en voor de donatie van extra buisjes bloed (inclusief arteriepunctie!). Zonder jullie hulp was er geen proefschrift geweest.

Mijn promotor, Prof. dr. P.W. de Leeuw. Beste Peter, ik heb in de afgelopen jaren ontzettend veel van jou mogen leren en ben erg blij dat jij mijn promotor was. Jouw unieke kijk op dingen zorgde ervoor dat ik weer een nieuwe weg in kon slaan als ik ergens op vastliep. De stukken werden hierdoor steeds vele malen beter. Ook jouw kundigheid in het schrijven van cover letters en kiezen van gepaste tijdschriften heb ik enorm gewaardeerd. De maanden dat jij mijn directe begeleider werd tijdens de eerste piek van de COVID-19 pandemie en jouw ondersteuning bij het finaliseren van mijn stukken zorgde ervoor dat ik, ondanks mijn dagelijkse zorg voor coronapatiënten, stappen vooruit zette in het finaliseren van mijn proefschrift. Heel veel dank voor alles.

Mijn copromotor, Dr. P.M. Stassen. Beste Patricia, ik leerde jou 5 jaar geleden kennen als copromotor waarbij ik in de afgelopen jaren ontzettend veel hulp van jou heb gekregen. Vanaf het begin stond jij dagelijks (en in het weekend!) voor mij klaar om me te begeleiden bij het opzetten van het onderzoek, het managen ervan, het interpreteren van de data en bij het schrijven van mijn stukken. Ik vind het nog steeds onvoorstelbaar hoe snel jij bent in het beantwoorden van jouw mails en correctie van de stukken, zeker omdat ik niet de enige promovendus was die je begeleidde. Ik heb heel veel van jou geleerd en ben je ontzettend dankbaar voor de intensieve en leuke begeleiding. Bij tegenslagen maakte jouw gevoel voor humor het altijd weer goed! Sinds 2019 ben jij tevens mijn opleider acute interne geneeskunde, een taak waar jij je eveneens volledig voor inzet. Bedankt voor alles. 
Mijn copromotor, Dr. J. Buijs. Beste Jacqueline, we kennen elkaar al bijna 10 jaar waarbij jij een belangrijke rol hebt gehad in mijn medische en wetenschappelijke opleiding. In 2011 was je mijn begeleider tijdens mijn coschap interne en werd mijn interesse voor de interne geneeskunde gewekt (waardoor ik mijn voorkeur voor cardiologie liet vallen). In 2012 werd jij mijn opleider interne geneeskunde in Atrium MC. Als opleider bood jij mij de kans om mijn opleiding twee jaar te onderbreken voor dit onderzoekstraject, welke door jou in samenwerking met Zuyderland MC werd mogelijk gemaakt. Bedankt voor de hulp bij het opzetten van het onderzoek, de studie management, het schrijven van de stukken en jouw uitgebreide correcties (zowel inhoudelijk als taalkundig). De stukken werden er vele malen mooier door. Ik heb in de jaren ontzettend veel van jou geleerd, zowel op internistisch als wetenschappelijk gebied. Heel veel dank hiervoor.

De leden van de beoordelingscommissie, Prof. dr. J.M.G.A. Schols, Prof. dr. O. Bekers, Prof. dr. F.H. Bosch, Prof. dr. W.F. Buhre, Prof. dr. B.M. Buurmans-van Es, bedankt voor de tijd die jullie genomen hebben voor de beoordeling van mijn proefschrift.

Mijn mede auteurs en onderzoekers: Sander, Arisja, Maarten, Dave, Paul, Daan, Lonne, Robin, Audrey, Sarah, Lars en Irene.

Beste Sander, bedankt voor je hulp en ondersteuning bij de statistische analyses en uitleg hiervan. Je maakte altijd tijd voor mij en voor een praatje over de stand van zaken van mijn proefschrift. Ik heb heel veel van je geleerd! Lieve Arisja, zonder jouw hulp bij de management van de RISE UP studie in MUMC+ had ik nooit de beschikking gehad over zo'n mooie dataset. Bedankt voor de gezelligheid tijdens onze wetenschappelijke meetings en als collega AIOS interne geneeskunde. Jammer dat je nu zover weg woont maar we houden contact! Beste Maarten, ontzettend bedankt dat je ons de mogelijkheid hebt geboden om de biomarkers te kunnen bepalen. Zonder jou hulp was dit niet mogelijk geweest. Ik kon altijd met al mijn vragen bij jou terecht wat ik enorm gewaardeerd heb. Beste Dave, jouw cardiologische visie verbeterde het stuk over MACE enorm. Je hebt ontzettend veel tijd gestoken in het beoordelen van alle ECGs waarvoor mijn dank. Ik vond het een heel fijne samenwerking! Beste Paul, ongelofelijk met wat voor tempo jij jouw promotietraject bent gestart. Bedankt voor je hulp bij de COVID-19 database, het analyseren van de data en schrijven van het artikel. Leuk dat jij nu je eigen promotie traject ingaat waarbij je sommige onderdelen van mijn proefschrift verder gaat bekijken. Ik kijk uit naar de resultaten! Beste Daan, bedankt voor het gebruik van de COVID-19 database van het Zuyderland $\mathrm{MC}$ en je bijdrage aan het artikel. Jouw hulp wordt enorm gewaardeerd. 
Beste Lonne, jij was de eerste WESP student die me kwam helpen bij de dataverzameling en het analyseren van de data rond het intuïtie project. Bedankt voor je enthousiasme, enorme inzet en gezellige gesprekken (over wetenschap, geneeskunde of het leven). Veel succes in je verdere carrière als spoedeisende hulp arts. Beste Robin, bedankt voor de hulp met het aanvullen van de database en bijdrage aan het biomarker stuk. Je was vanaf het begin al geheel zelfstandig (wat wel moest omdat ik zo nodig enkele weken op vakantie ging). Leuk dat ik je nadien ook als collega assistent interne heb leren kennen. Veel succes en plezier in je verdere carrière als huisarts! Beste Audrey, als AKO student met gecombineerde stage cardiologie en wetenschappelijke stage bij mij, heb jij je voornamelijk beziggehouden met het MACE project waarvoor ik je wil bedanken. Je hebt er veel werk aan gehad. Veel succes met je verdere loopbaan als huisarts. Beste Sarah, bedankt voor jou hulp bij het 'concern' project, het is mede dankzij jou een mooi stuk geworden. Succes in je verdere loopbaan als arts. Beste Lars, bedankt voor al jouw hulp de afgelopen maanden met betrekking tot verschillende onderzoeksprojecten en de implementatie van het RISE UP model. Jij en Irene waren mijn steun en toeverlaat bij het opzetten van het COVID-19 onderzoek. Bedankt ook voor alle fijne gesprekken, ik vond het een erg fijne samenwerking. Heel veel succes met het afronden van je eigen proefschrift.

Opleiders, Prof. dr. C.D.A. Stehouwer en Prof. dr. R.P. Koopmans, bedankt voor de kans om mijn opleiding tot internist te onderbreken voor dit wetenschapstraject.

De internisten en $M D L$-artsen van het Zuyderland $M C$ en $M U M C+$. Bedankt dat jullie mij de kans hebben gegeven om bij jullie patiënten onderzoek te doen. In bijzonder wil ik Cees van Deursen, Kim Hurkens en Evelien Pijpers bedanken voor hun hulp bij de METC aanvraag en bijdrage aan het studie protocol.

Verder wil ik graag collega AIOS, SEH artsen en internisten bedanken voor deelname aan de studie waaronder Aimée, Anouk, Arnée, Benedict, Carmen, Carine, Dewa, Eline, Jalil, Jana, Jeantine, Judith, Lian, Limmie, Liza, Lotte, Maaike, Mark, Marc, Maurice, Michèle, Michelle, Renée, Roel, Rory, Sanne, Saskia, Sema, Sharona, Tessa, Tom, Vera en Wenke uit Zuyderland MC en Aniek, Anne, Avalon, Bart, Dorien, Elif, Fabienne, Heiko, Ivette, Krista, Lara, Laura, Lieselot, Marjolein, Mark, Maroeska, Martijn, Merel, Michelle, Nicole, Papay, Paulien, Roel, Roderick en Yvo uit MUMC+. Ik weet dat het voor jullie niet altijd een makkelijke taak was patiënten te includeren, zeker niet op momenten dat de SEH overliep van drukte. Desondanks hebben jullie je allemaal voor dit onderzoek ingezet en ik ben jullie erg dankbaar hiervoor. 
Internisten van de vakgroep acute geneeskunde Hella Borggreve, Ronald Henry, Dirk Posthouwer en Roger Rennenberg. Bedankt voor jullie interesse en feedback op mijn onderzoek. En natuurlijk bedankt voor de leuke en leerzame begeleiding van mij als fellow acute geneeskunde.

Mede-fellows acute geneeskunde, Dewa, Hajar, Judith, Maaike, Nicole en Yvonne, bedankt dat jullie er altijd voor mij (en elkaar) zijn én voor het feit dat jullie de werkvloer vele malen gezelliger maken! Dewa, jij bent zelf ook met je promotietraject bezig en kon als geen ander begrijpen wat dit naast je werk en gezin inhield. Ondanks je bezigheden maakt je altijd tijd voor mij vrij om even te kletsen of dingen te bespreken. Ik snap soms niet hoe het je het allemaal voor elkaar krijgt: een gezin, promotie traject, opleiding en nu een eigen studenten uitzendbureau, chapeau! Veel succes bij het afronden van je proefschrift. Hajar, je ben onderhand al bijna 1 jaar mede-fellow maar helaas hebben we nog niet heel veel kunnen samenwerken. Desalniettemin spreken we elkaar regelmatig en zullen we in de toekomst vast vaker samen op de SEH staan. Succes met je verdere opleiding. Judith, jij was sinds 2013 mijn mede-AIOS interne geneeskunde in het Zuyderland MC en nu mijn mede-fellow acute geneeskunde. In de afgelopen jaren heb je leren kennen als een betrokken en zorgzame collega. Bedankt voor alle gezelligheid, zeker ook tijdens alle fellowdagen. Over enkele maanden ben jij al klaar als acuut internist en ik wens je heel veel succes met het vinden van je droombaan! Hopelijk zullen we elkaar in de toekomst nog regelmatig zien. Maaike, ik ken jou het langst van alle fellows maar de laatste 2 jaar staan we eigenlijk pas regelmatig samen op de werkvloer. Wat heb ik jou leren kennen als een lieve en betrokken collega. Jouw nuchterheid, collegialiteit en het feit dat ik altijd bij je terecht kan, kan ik enorm waarderen. Bedankt voor alle gezellige gesprekken en alle keren dat je de kliniek overnam zodat ik aan mijn onderzoek kon werken. Heel veel succes op de infectieziekten en je IC opleiding. Nicole, jij bent nu al klaar als internist acute geneeskunde en bent nu officieel mijn baas. We hebben de afgelopen jaren niet vaak samen gestaan maar desondanks kon ik altijd voor een praatje of advies bij je terecht. Heel veel succes in je verdere carrière als acuut internist. Yvonne, ook jij bent klaar met de opleiding en werkzaam als internist acute geneeskunde. Toen ik net begon als fellow had jij de supervisie op de SEH wat ik als heel fijn ervaren heb. Altijd kon ik voor vragen en advies bij je terecht en dit maakte de SEH een stuk gezelliger! Heel veel succes in je verdere carrière.

Mijn mede AIOS en onderzoeksbuddies van de interne geneeskunde Ankie, Ellis, Monica en Stijn. Ankie, samen met Ellis zijn we samen begonnen als AIOS interne waarna jij al snel koos voor de MDL. Net als Stijn ging jij er 2 jaar tussenuit om 
onderzoek te doen. Voor je hulp bij de METC aanvraag ben ik je enorm dankbaar. Je bent nu bijna klaar met je promotietraject en ik kijk uit naar je verdediging. Bedankt voor alle gezelligheid op de werkvloer en buiten het werk om. We zijn nu al eeuwen bezig om een afspraak te plannen met onze mannen en kindjes, hopelijk kunnen we snel een datum vinden. Ellis, toen ik net begonnen was als AIOS stond ik met jou samen op de MDL. Wat hebben we samen een ongelofelijke lol gehad tijdens die stage (zo erg dat de bazen onze werkplek als kippenhok bestempelde!). Sindsdien hebben we altijd even veel lol als we samenkomen en raken we niet uitgekletst. Bedankt voor al jouw gezelligheid, je luisterend oor en adviezen (ook met betrekking tot wetenschap). De laatste tijd hebben we elkaar weinig gezien, maar we plannen gauw een datum. Monica, we kennen elkaar al vanaf het moment dat jij semi-arts was in het voormalige Atrium MC waar ik toen mijn coschap liep. Sindsdien hebben we nog vaker mogen samenwerken. Altijd als we elkaar zien maken we tijd om even bij te kletsen. Bedankt voor al het wetenschappelijk advies wat je me de afgelopen jaren hebt gegeven. Ik wens je veel succes met het afronden van je opleiding. Stijn, ook jou ken ik vanuit mijn coschap interne geneeskunde in het voormalige Atrium MC. Bedankt dat jij de weg hebt vrijgemaakt voor een 'tweejarig' onderzoekstraject dat ik nadien ook heb kunnen volgen. Veel succes met je verdere carrière als endocrinoloog.

Mede AIOS uit Zuyderland MC en MUMC+, bedankt voor alle gezelligheid, collegialiteit en leuke uitjes. Dit maakt het combineren van opleiding en een promotietraject een stuk gemakkelijker.

Mijn lieve vriendinnetjes Danielle, Jolanda, Lucy en Stefanie. Al sinds de middelbare school zijn we samen een hecht clubje en hebben we meerdere feestjes en gekkigheden samen beleefd! Onze reis naar Parijs is al veel te lang geleden maar deze kan ik me nog goed herinneren. Door werk, gezin en drukte zijn we niet heel vaak met zijn vijven maar als we samen afspreken dan is het net als vanouds. Ik kan altijd bij jullie terecht voor zowel leuke als minder leuke dingen, dit waardeer ik enorm. Bedankt voor alle gezellige uitjes, etentjes en stapavondjes. Ik hoop dat we de komende jaren nog heel veel gezellige uitjes beleven! Stefanie, bedankt voor alle gezellige samenkomsten, kroegentochten en onze relaxuitjes naar de sauna en Mallorca. Deze uitjes zorgde ervoor dat ik mijn rust kon vinden in drukke periodes. Hopelijk volgen er nog veel meer van deze gezellige en relaxte uitjes in de toekomst! Lieve vrienden, Lars en Natalia, Michel en Djalisah, Maggy en Roger, Famke en Marc, met jullie heb ik al heel veel gezellige avonden, feestjes en vakanties mogen meemaken. Zo was er de legendarische vakantie met Lars en Natalia in Schloss Holte die we nooit zullen (en kunnen!) vergeten. Michel en Djalisah, ik leerde jullie kennen 
als overburen en vlak na deze ontmoeting hadden we al plannen om samen op vakantie te gaan. Onze reis naar het Dominicaanse Republiek is er een om nooit te vergeten! Hopelijk kunnen we deze reis nog een keer herhalen, met of zonder kinderen. Maggy, Roger, Famke en Marc, tijdens onze reizen naar de Ardennen en Rome heb ik heel veel geleerd. Dat je best geen zwerfhonden oppakt, dat je Rome het beste kunt bekijken vanuit een taxi én je je vluchttijden op tijd checkt (sorry guys!). Hopelijk durven jullie nog een keer met mij samen op vakantie te gaan.

Beste Wim en Ria, mijn oom en tante, bedankt voor jullie gastvrijheid en jullie interesse in mijn onderzoek. Hopelijk kunnen jullie bij de verdediging zijn.

Beste Leon en Riet, mijn oom en tante, ook al zie ik jullie niet heel vaak, jullie zijn altijd even geïnteresseerd in mijn leven. Wat leuk dat Leon wil deelnemen in mijn promotiecommissie. Tot dan.

Lieve tante Finie, bedankt voor jouw interesse in mijn opleiding tot internist. Jij kon goed begrijpen wat dit inhield omdat je zelf in de zorg gewerkt hebt. Ik vind het heel speciaal dat jij samen met mij de kaft van dit proefschrift wilde vormgeven, waarvoor heel veel dank.

Lieve Patrick, Sabine, Joppe en Sofie, mijn schoonfamilie, bedankt voor alle gezellige afspraken en feestjes. Ik kijk uit naar nog meer gezellige bijeenkomsten in de toekomst.

Beste Peter en José, mijn schoonouders, bedankt voor al jullie hulp en steun in de afgelopen jaren en alle keren dat jullie extra moesten komen oppassen zodat ik aan mijn onderzoek kon werken of naar mijn werk toe kon. De kindjes vinden het altijd geweldig als jullie komen en wij ook!

Maartje, mijn lieve zus, ook al zijn wij op sommige vlakken verschillend, toch lijken we in vele opzichten erg veel op elkaar en hebben we samen ontzettend veel lol. Of het nu uitjes met de kids, carnaval of concerten betreft, er wordt altijd heel veel gelachen. Ondanks je vele diensten en drukke gezinsleven vind jij altijd de tijd om even bij te kletsen of om de meest mooie creaties te maken zoals knutselwerkjes, carnavalskleding of leuke creatieve cadeautjes. Ik vraag me dan ook regelmatig af hoe je dat nou allemaal doet. Ik heb altijd veel van je kunnen leren en je staat altijd voor mij klaar waarvoor ik je ontzettend dankbaar ben! Ik vind het super leuk en bijzonder dat 
je nu mijn paranimf bent. Bedankt voor je hulp bij het afronden van dit proefschrift. Ik hoop dat we in de toekomst nog heel veel samen zullen lachen!

Jorrit, mijn broertje, jouw afwezigheid herinnert mij elke dag eraan om het maximale uit mijn leven te halen. Ook al kan je er niet bij zijn, in mijn gedachte ben je altijd bij mij.

Papa en Mama, zonder jullie hulp was ik nooit zover gekomen in mijn leven. Jullie geloofde altijd in mij en verzekerde mij ervan dat ik ondanks mijn problemen met taal er wel zou komen! Jullie stonden altijd voor mij klaar, zorgde dat ik alle kansen had om mij te ontwikkelen en leerde mij de belangrijkste normen en waarden in het leven. Ook voor jullie kleinkinderen staan jullie altijd klaar en wat zijn die 6 rakkertjes dol op jullie! Bedankt voor alle keren dat jullie extra hebben opgepast zodat ik aan dit proefschrift kon werken. Zonder jullie hulp was het mij nooit gelukt, ik hou van jullie! Papa, bedankt dat jij mijn paranimf bent, dit vind ik heel speciaal. Hopelijk kunnen we gauw weer samen een rondje fietsen. Mama, bedankt voor jouw interesse in mijn promotietraject en al jouw hulp de afgelopen jaren! We gaan gauw samen met Maartje een weekendje weg.

Timmy, mijn levenspartner en allerbeste vriend, ik kan me een leven zonder jou niet voorstellen. Jouw humor, enthousiasme en eeuwig optimisme maken mijn leven vele malen mooier. Deze eigenschappen maken van jou ook de perfecte papa en zorgde ervoor dat ik naast mijn opleiding en gezin dit promotie traject kon afronden. Bedankt voor alle keren dat je met Tuur op pad ging zodat ik aan mijn proefschrift kon werken en alle zorgen die jij draagt voor het gezin als ik aan het werk ben. Bedankt voor alle mooie momenten samen, het delen van lief en leed en al onze mooie reizen die we samen hebben kunnen maken (waarvan een belangrijk deel op de fiets). Ik hoop dat we nog heel veel tijd samen kunnen genieten en kijk uit naar nog meer mooie reizen in de toekomst. Ik hou ontzettend veel van jou!

Mijn lieve lieve kindjes, Tuur, Ize en Sepp. Tuur, wat ben je toch een enthousiast, energiek en slim mannetje! Wat kan ik toch genieten van ons samenzijn en jouw ongekende levenslust. Bedankt dat je altijd zo begripvol was als mama weer aan haar boekje moest gaan werken. Nu het boekje klaar is, kunnen we weer veel vaker samen op pad gaan! Ize en Sepp, dat jullie besloten samen op de wereld te komen was een van de grootste verrassingen in mijn leven. Het is ontzettend bijzonder hoe jullie als tweeling opgroeien. Ize mijn vrolijke, lieve en stoere meisje en Seppie mijn vrolijke, eigenwijze en zorgzame mannetje. Bedankt dat jullie 's middags zo uitgebreid sliepen 
zodat mama aan haar proefschrift kon werken. Lieve Tuur, Ize en Sepp, bedankt dat jullie mij elke dag herinneren aan wat echt belangrijk is in het leven. Jullie maken mij elke dag ontzettend trots en gelukkig en ik ben zo blij dat ik jullie mama mag zijn! Ik kijk uit naar alle momenten die we nog samen gaan beleven. Ik hou ontzettend veel van jullie! 
Curriculum Vitae 



\section{Curriculum Vitae}

Noortje Zelis was born on December 12, 1985 in Heerlen, the Netherlands. She graduated from secondary school at Bernardinus College, Heerlen, the Netherlands in 2005. Subsequently, she started with a bachelor in Biomedical Sciences at the Catholic University of Leuven, Belgium. In 2006 she transferred to medical school at the Catholic University of Leuven and obtained her bachelor's degree in 2008 (cum laude) and master's degree in 2012 (cum laude). During her master she assisted in a study at the haematology department in the university hospital of Leuven under supervision of Prof. dr. M. Delforge. In September 2012 she started her specialisation in internal medicine in the Atrium Medical Centre in Heerlen under supervision of dr. J. Buijs. Her $\mathrm{PhD}$ trajectory at the research departments of internal medicine in Zuyderland $\mathrm{MC}$ and Maastricht Univeristy Medical Centre+, under supervision of Prof. dr. P.W. de Leeuw, dr. P.M. Stassen and dr. J. Buijs, started in May 2016. In 2018 she continued her residency internal medicine in Zuyderland Medical Centre under supervision of $\mathrm{dr}$. J. Buijs and dr. F. Stifft. Since 2019 she started her differentiation in acute internal medicine in Maastricht University Medical Centre+ under supervision of dr. P.M. Stassen and Prof. dr. R.P. Koopmans. 

Scientific output 



\section{Scientific output}

\section{Published papers}

\section{This thesis}

Study protocol for a multicentre prospective cohort study to identify predictors of adverse outcome in older medical emergency department patients (the Risk Stratification in the Emergency Department in Acutely III Older Patients (RISE UP) study). Zelis N, Buijs J. de Leeuw PW, van Kuijk SMJ, Stassen PM. BMC Geriatrics 2019;19(1):65

Short-term mortality in older medical emergency patients can be predicted using clinical intuition: A prospective study. Zelis N, Mauritz AN, Kuijpers LIJ, Buijs J, de Leeuw PW, Stassen PM. Plos one 2019; 14(1):e0208741

Concerns of older patients and their caregivers in the emergency department. Zelis $\mathbf{N}$ Huisman SE, Mauritz AN, Buijs J, de Leeuw PW, Stassen PM. Plos One 2020;15(7):e0235708

A new simplified model for predicting 30-day mortality in older medical emergency department patients: The rise up score. Zelis N, Buijs J, de Leeuw PW, van Kuijk SMJ, Stassen PM. European Journal of Internal Medicine 2020;77:36-43

Do biomarkers add anything to clinical prediction of mortality in older medical emergency department patients? Zelis N, Buijs J, de Leeuw PW, Stassen PM, RISE UP investigator group. European Journal of Internal Medicine 2020; S09536205(20)30299-5. Online ahead of print.

\section{Other publications}

Treatment with lenalidomide (Revlimid ${ }^{\circledR}$ ), cyclophosphamide (Endoxan ${ }^{\circledR}$ ) and prednisone (REP) in relapsed/refractory multiple myeloma patients: results of a single centre retrospective study. Zelis $\mathbf{N}$, Devos T, Dierickx D, Janssens A, Raddoux J, Verhoef G, Delforge M. Acta Clinica Belgica 2014; 69(2):98-103

CT in relation to RT-PCR in diagnosing COVID-19 in The Netherlands: A prospective study. Gietema HA, Zelis N, Nobel JM, Lambriks LJG, van Alphen LB, Oude Lashof AML, Wildberger JE, Nelissen IC, Stassen PM. PLoS One 2020;15(7):e0235844 


\section{Submitted papers}

The value of biomarkers in predicting mortality in older medical emergency department patients. Zelis N, Hundscheid R, Buijs J, de Leeuw PW, Raijmakers MTM, van Kuijk SMJ, Stassen PM.

Major adverse cardiovascular events in older emergency department patients presenting with non-cardiac medical complaints. Zelis $\mathbf{N}$, Roumans-van Oijen AMM, Buijs J, van Kraaij DJW, van Kuijk SMJ, de Leeuw PW, Stassen PM.

RISE UP score: predicting prognosis in patients with COVID-19 in the emergency department. Van Dam PMEL, Zelis N, Stassen PM, van Twist DJL, de Leeuw PW, van Kuijk SMJ, Buijs J.

\section{Oral presentations}

NIV Internistendagen 2019 (Maastricht, the Netherlands): Prediction of 30-day mortality in older medical emergency department patients. (award)

SAMsterDAM2 (Amsterdam, the Netherlands): Disease perception and clinical impressions of patients, nurses and physicians predict 30-day mortality and other adverse outcomes in older emergency patients. (second best oral presentation)

\section{Poster presentations}

SAMsterDAM2 (Amsterdam, the Netherlands): Disease perception and clinical impressions of patients, nurses and physicians predict 30-day mortality and other adverse outcomes in older emergency patients.

$8^{\text {th }}$ scientific symposium Zuyderland MC (Sittard-Geleen, the Netherlands): Routine lactate levels are predictive for mortality and adverse outcome in elderly emergency patients. 

